PhD Dissertation

\title{
Gap Waveguide Array Antennas and Corporate-Feed Networks for mm-Wave band Applications
}

\author{
Universitat Politècnica de València \\ Departamento de Comunicaciones
}

\author{
Author \\ Miguel Ferrando Rocher \\ Advisors \\ Prof. Dr. Alejandro Valero Nogueira \\ Dr. Jose Ignacio Herranz Herruzo
}

Valencia, December 2018 

"Just when you think you know all the answers, the universe changes all the questions." 


\section{Agradecimientos}

Uno recuerda muchas más cosas de las que la gente supone, yo, por ejemplo, no pasa un mes sin que recuerde aquel primer día en el iTEAM.

Toda mi vida ha estado de algún modo relacionada con la Universidad, los motivos son evidentes. Recuerdo una pequeña rampa de acceso al parking de la UPC en Barcelona, y recuerdo cuando más allá de la ETSIT en Valencia no había un pabellón deportivo sino unas cuantas pistas de cemento y después la huerta. Puede parecer absurdo hablar de todo esto en una sección de agradecimientos, pero no podría hacerlo de otra manera.

Este viaje ha sido largo. No han sido los últimos 4 años, ha sido toda mi vida. Los libros de Electromagnetismo en la estantería mientras escribía, y yo tomando a aquellos autores como protagonistas de mis historias.

Sois muchos los compañeros que me habéis acompañado en esta nueva etapa de mi vida.

Alejandro y Jose Ignacio gracias por ser los mejores directores de tesis que uno podría tener. Rigurosos en el trabajo, me habéis ayudado, apoyado, animado, enseñado. Enseñado mucho. Gracias por guiarme, por no ser simples tutores o directores sino mentores. Un mentor es un maestro y yo creo que ese término (a veces tan mal usado) es el que mejor os define. Os agradezco infinitamente haber apostado por mí para esta larga andadura. Haberme corregido siempre en favor de un aprendizaje. Haberme dejado espacio para que pudiera llevar a cabo mi investigación y, a la vez, haber estado siempre dispuestos cuando lo he necesitado. Sin duda mi mayor deseo es que este trabajo conjunto sea sólo una muesca más en nuestro camino.

Tantos compañeros, amigos en el grupo. Toni y Bernat, de vosotros he aprendido que a veces lo imposible es posible y también que un pin de 100 micras se ve fabuloso en el ordenador, pero luego la fresadora tenía sus límites.

Me gustaría especialmente agradecer su paciencia infinita a Dani. Qué suerte tiene este grupo de tenerte en la esquina, en la base central de mando: controlando, asesorando, aconsejando y ayudando 
a todo el mundo. En cualquier minuto y siempre sin un pero. Es un placer haber viajado contigo, compartido aventuras en Londres, Paris, Boston...y haber aprendido tanto de ti. También a Nora y María, las chicas de la sala del GRE. Vosotras sois parte imprescindible de este trabajo, hemos crecido juntos. Muchas gracias por todos los momentos de distensión, las risas, por apoyarme con la tesis y también con el teatro.

Mariano, Eva, Vicent M., Marta, Lorenzo, Juan, Felipe. Conoceros aún más y aprender de vosotros ha sido un privilegio. Gracias, además, por haberme permitido compartir docencia con vosotros. Han sido momentos para crecer tanto profesional como vitalmente.

Gente que va y viene. Que de algún modo siempre está. Marc, Cristina, Alexia, Jaime, por supuesto Toni B. y Tomás. Que suerte haberos conocido y que pena no haber compartido más tiempo. Sois personas brillantes y con mucha luz, ojalá pudiera robaros algo de ese magnetismo que transmitís, y ojalá, de la manera que sea, volvamos a encontrarnos.

A la familia de Ecuador: Fernando, Diana, Carlos, Daniel, Anibal. A la de Marruecos: Zakaria, Hamza, Yousra. A la de Argentina: Juan Pablo, Alberto, Guillermo y tantos otros que seguro que olvido. También parte de este trabajo ha sido posible gracias a Ashraf, Jian, Abbas, Sadegh y Jin Lin, que fueron un apoyo fundamental durante mi estancia en Chalmers University of Technology.

A mi familia. A mis abuelos Rosita y Miguel, Amparo y Salvador. Sé que habéis estado conmigo.

A mi hermana Nuria, por aguantar mil cenas oyéndonos hablar de Antenas.

A mi madre, por apoyarme y espolearme. Por exigirme siempre más cuando yo creo que es suficiente.

A mi padre, por ser un guía infatigable, porque llegar hasta aquí a tu lado es de las mejores cosas que haré en esta vida.

A Dulce, la mujer más extraordinaria del mundo. Si existe el destino, hizo bien al ponernos juntos. Tu a mi lado y yo al tuyo para superar todos los obstáculos y superar todas las barreras. Nunca habrá suficientes gracias por haberme apoyado tanto y haber sido tan paciente y sincera en cada etapa de este viaje.

Es curioso como al final de todo, de tanto trabajo, debería sentirme inmensamente feliz, pero echando la vista atrás, no soy más feliz ahora que durante todo el camino recorrido a vuestro lado. Gracias. 


\begin{abstract}
This thesis deals with topics of special interest regarding the design of antennas at the mm-wave band. Today, implementing passive components that operate in the mm-wave band and to ensure the appropriate metallic contact is challenging. Commonly, conventional planar transmission lines and hollow metallic waveguides are the usual solutions but they present high losses or they do not ensure a good metallic contact. So, new concepts must be explored.

Gap Waveguides (GWs), result suitably since they do not require metallic contact for shielding. Antenna arrays in Gap Waveguide Technology (GW) emerges as one promising candidate to naturally meet some of the mentioned needs. GW technology has demonstrated to be effective for mm-wave band devices because it enables full-metal distribution networks in a much simpler way than conventional waveguides. Very low distribution losses can be achieved preserving at the same time the assembly simplicity of multilayer microstrip feeding networks. This unique feature is a consequence of gap waveguides ability to safely confine the electromagnetic wave propagation through a contactless structure.

During the last decade, there have been important advances in GW technology and a good number of gap waveguide-based arrays can be found in the literature. This thesis goes a step further in the contribution to mm-wave gap waveguide antennas. Here, antennas with linear polarization as well as circular or dual polarization are proposed. Dual band antennas has also been explored. These contributions have been carried out with a focus on satellite communications on-the-move. In addition, new distribution networks have also been explored to obtain more compact, low-profile and lighter antennas.
\end{abstract}




\section{Resumen}

Esta tesis aborda temas de especial interés en el diseño de antenas en la banda de milimétricas. Hoy en día, implementar componentes pasivos para operar en la banda de milimétricas y asegurar el contacto y el alineamiento metálico apropiado entre piezas, resulta un desafío complejo. Habitualmente las líneas de transmisión y las guía de ondas metálicas son las soluciones adoptadas, pero en el primer caso se presentan pérdidas al ser soluciones impresas y en el segundo caso un mal contacto metálico conlleva fugas de campo. Por tanto, se están explorando nuevos conceptos que solucionen estos problemas.

La tecnología Gap Waveguide (GW) resulta adecuada ya que no requiere de contactos metálicos. En los últimos años han surgido las agrupaciones de antenas basadas en la tecnología Gap Waveguide y son un candidato prometedor para satisfacer algunas de las necesidades mencionadas. La tecnología GW ha demostrado ser atractiva para dispositivos de milimétricas porque permite redes de distribución completamente metálicas de una manera más simple que las guías de onda convencionales. Por tanto, estas redes tienen muy bajas pérdidas pero además son simples de fabricar. Esto es posible gracias a la capacidad de las GW de confinar de forma segura la propagación de ondas electromagnéticas por medio de una estructura que no requiere de contacto.

Durante la última década, se han hecho avances importantes en la tecnología GW y en la literatura se pueden encontrar un buen número de antenas basadas en GW. Esta tesis va un paso más allá en la contribución de este tipo de antenas. Aquí, no solo se presentan antenas con polarización lineal, como suelen ser las desarrolladas hasta ahora, sino también con polarización dual, circular y duales en banda. Estas aportaciones son especialmente atractivas dentro del campo de las comunicaciones por satélite en movimiento (SATCOM on-the-move). Además se han explorado nuevas redes de distribución que permiten antenas planas más compactas, más ligeras. 


\section{Resum}

Esta tesi aborda temes d'especial interés en el disseny d'antenes en la banda de mil-limètriques. Hui en dia, implementar components passius per a operar en longituds d'onda tan xicotetes (de l'orde de mil-límetres) i assegurar el contacte i l'alineament metàllic apropiat entre peces, resulta un desafiament complex. Habitualment les línies de transmissió i les guia d'ones metàl-liques són les solucions adoptades, però en el primer cas es presenten pèrdues al ser solucions impreses i en el segon cas un mal contacte metàl-lic comporta fugues de camp. Per tant, s'estan explorant nous conceptes que solucionen estos problemes.

La tecnologia Gap Waveguide (GW) resulta adequada ja que no requerix de contactes metàl-lics. En els últims anys han sorgit les agrupacions d'antena basades en la tecnologia Gap Waveguide i són un candidat prometedor per a satisfer algunes de les necessitats mencionades. La tecnologia GW ha demostrat ser atractiva per a dispositius de banda d'ones mil-limètriques perquè permet xarxes de distribució completament metàl-liques d'una manera més simple que les guies d'onda convencionals. Per tant estes xarxes tenen baixes pèrdues peró, a més, són simples de fabricar. Açò és possible gràcies a la capacitat de les GW de confinar de forma segura la propagació d'ones electromagnètiques per mitjà d'una estructura que no requerix de contacte.

Durant l'última dècada, s'han fet avanços importants en la tecnologia GW i en la literatura es poden trobar un bon nombre d'antenes basades en GW. Esta tesi va un pas més enllà en la contribució d'este tipus d'antenes. Ací, no sols es presenten antenes amb polarització lineal com solen ser les desenrotllades fins ara, sinó també antenes amb polarització dual, circular i inclús antenes duals en banda. Estes aportacions són especialment atractives dins del camp de les comunicacions per satèl-lit en moviment (SATCOM on-themove). A més també s'han explorat noves xarxes de distribució que permeten obtindre antenes planes més compactes, més lleugeres. 


\section{Table of contents}

1 Introduction $\quad 1$

1.1 Millimeter Wave Band . . . . . . . . . . . . . . . . . . 3

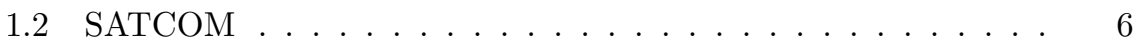

1.2.1 Gap Waveguide Technology . . . . . . . . . . . . 19

1.3 Objectives . . . . . . . . . . . . . . . . . . . . 32

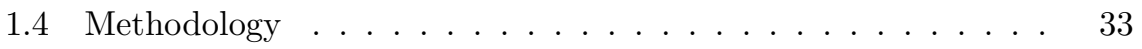

1.5 Structure of the Thesis . . . . . . . . . . . . . . . . 34

2 Corporate feeding networks based on Gap Waveguide $\quad 37$

2.1 Bed of nails . . . . . . . . . . . . . . . . . 37

2.2 Other AMC Realizations . . . . . . . . . . . . . . . . . 44

2.3 Contactless feature of $\mathrm{GW} \ldots \ldots . \ldots 51$

2.4 GGW Corporate-feed networks ............. . . 55

2.4.1 H-plane coupling layer . . . . . . . . . . . . . 55

2.4.2 H-plane feeding networks in GGW . . . . . . . . . . 57

2.4.3 E-plane feeding networks in GGW . . . . . . . . . . 59

2.5 RGW corporate-feed networks . . . . . . . . . . . . 62

2.6 GGW-RGW corporate-feed networks . . . . . . . . . . . 65

3 Radiating elements for Gap Waveguide Arrays $\quad 73$

3.1 Slots fed by Coaxial Cavities . . . . . . . . . . . . . . 75

3.1.1 Coaxial Cavities in Gap Waveguide . . . . . . . . . . . 77

3.1.2 Groove Gap Waveguide feeding . . . . . . . . . . . . . . 79

3.1.3 Ridge Gap Waveguide feeding . . . . . . . . . . . . 83

3.1.4 GGW-RGW feeding . . . . . . . . . . . . . 85

3.1.5 Dual-Band Antennas . . . . . . . . . . . . . . 86

3.2 Cylindrical Cavities . . . . . . . . . . . . . . . . . . . 93

3.2.1 Cylindrical Cavities fed by GGW . . . . . . . . . . . . 96

3.2.2 Cylindrical Cavities fed by RGW . . . . . . . . . . . . . . 97

3.2.3 Chamfered Cylindrical Cavities . . . . . . . . . 100 
3.2.4 Dual-Polarized Cavities . . . . . . . . . . . . . . 101

3.2.5 Dual-Band Cavities . . . . . . . . . . 103

4 Gap Waveguide Array Antennas 109

$4.116 \times 16$ Single-Layer Array Antenna in V-band . . . . . . . . . . 110

$4.28 \times 8$ Single-Layer Cavity-Backed Slot Array Antenna . . . . . . 121

$4.34 \times 4$ Single-Layer Circularly-Polarized Array Antenna . . . . . 129

$4.48 \times 8$ Dual-Polarized Array Antenna . . . . . . . . . . . . . . . . 137

4.5 $8 \times 8$ Single-Layer Dual-Band Cavity-Backed Slot Array Antenna 148

4.6 $8 \times 8$ Dual-Band Array Antenna using Cylindrical Cavities . . . 157

5 Conclusions and Future Work 163

A Technical Results $\quad \mathbf{1 6 7}$

A.1 JCR Indexed journals . . . . . . . . . . . . . . . . . . . . . 167

A.2 International Conferences . . . . . . . . . . . . . . . . . 168

A.3 National Conferences . . . . . . . . . . . . . . . . . . . 170

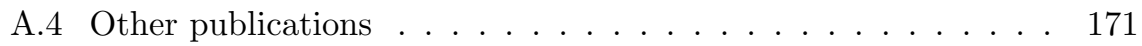

A.4.1 JCR Indexed journals . . . . . . . . . . . . . . . . 171

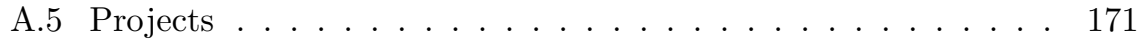

A.6 Patents . . . . . . . . . . . . . . . . . . . . 172

A.7 Awards . . . . . . . . . . . . . . . . 172

$\begin{array}{lr}\text { References } & 173\end{array}$ 


\section{Chapter 1}

\section{Introduction}

"A lot of people want a shortcut. I find the best shortcut is the long way, which is basically two words: work hard."

— Randy Pausch

Today, wireless connectivity is considered to be an essential factor for assessing the quality of life as wireless communication systems continue to drive human productivity and innovation in many areas. Communication at millimeterwave frequencies can be considered as the most recent game-changing development for wireless systems. Thus, the mm-wave band is called to allocate new generation communication services as an additional spectrum to the microwave band.

The millimeter wave spectrum $(30-300 \mathrm{GHz})$ is of increasing interest to service providers and systems designers because of the wide bandwidths available for carrying communications. Such wide bandwidth is valuable in supporting applications such as high-speed data transmission and video distribution. Planning for millimeter wave spectrum usage must take into account the propagation characteristics of radio signals at this frequency range. While signals at lower frequency bands can propagate for many kilometres and penetrate more easily through buildings, millimeter wave signals can travel only a few kilometres or less and do not penetrate solid materials easily. However, these characteristics of millimeter wave propagation are not necessarily disadvantageous. Millimeter waves can permit more densely packed communications links, thus providing very efficient spectrum utilization, and they can increase the security of communication transmissions [1]. 


\section{CHAPTER 1. INTRODUCTION}

Antenna systems with high performance will be required at these bands to compensate for the strong propagation losses. There are plenty of technical issues and mechanical challenges in implementing millimeter-wave front-ends. These factors are cost pressure, compact size requirement, improved system integration density, packaging and cross-talk suppression, lower DC power consumption and lower RF power dissipation.

Conventional rectangular waveguides, planar transmission lines such as coplanar waveguides or microstrip lines are well-characterized transmission media which are used in a variety of complex RF component and circuits until today. However, there are some factors to be considered while using these conventional technologies at millimeter-wave frequencies or above, particularly for low-cost RF applications. Classical waveguide technology is well known to provide the lowest losses and very high power handling capability. For these reasons, it is often used in space applications. Unfortunately, this technology is not flexible enough to manufacture intricate components (such as an antenna corporate-feed network), mainly because it is critical to ensure good electrical contact when it is manufactured in several pieces. The connection between the split blocks can be achieved by screwing, dip-brazing techniques or more sophisticated (and less massive) methods such as diffusion bonding. These techniques are costly, complicated and often not scalable to higher frequencies. As the operation frequency approaches millimeter-wave frequencies, the physical dimensions of the components decrease, and a high level of precision is required for manufacturing and assembling the metal pieces by conventional methods [2].

On the other hand, microstrip and coplanar lines are the most representative planar transmission lines. These are robust, low-cost solutions and very suitable for integrating active microwave components on circuit boards. However, the transmission properties of both microstrip and coplanar lines greatly depend on the substrate parameters. Both lines suffer from high insertion loss at mmwave band due to the presence of lossy dielectric material, as well as significant conductor losses caused by the small dimensions. Additionally, the excitation of unwanted surface waves and propagation via substrate modes is another severe problem of printed technology. Therefore, there is a definite need for new RF technology (in particular at $30 \mathrm{GHz}$ and above) which will allow lowcost manufacturing of massive commercial applications.

Alternatives have been developed already to replace the two main conventional technologies (PCB technology and hollow waveguide technology). One of these alternative technologies is the Substrate Integrated Waveguide (SIW), despite it still suffers from high dielectric losses at millimeter wave frequency range. In this regard, recently developed gap waveguide becomes an innovative technology well suited for millimeter-wave RF applications. It is based on Prof. Kildal's invention in 2009, described in the introductory papers [3], 
[4]. These papers describe several types of gap waveguides that can replace microstrip technology, coplanar waveguides, and standard rectangular waveguides in millimeter wave RF systems and antennas.

The gap waveguide can be a low loss RF building block having significant advantages in manufacturing waveguide components such as antennas or filters and at the same time integrating RF electronics with waveguide components.

\subsection{Millimeter Wave Band}

Millimeter wave spectrum (Fig. 1.1) can be used for high-speed wireless communications. Regulatory agencies and researchers are considering it as the way to bring " $5 \mathrm{G}$ " into the future by allocating more bandwidth to deliver faster, multimedia content and services and higher-quality video. The European Telecommunications Standards Institute (ETSI) itself defines the following qualities of the mm-wave spectrum [5]:

- Lots of spectrum available: There is one order of magnitude of more spectrum available in this band than in lower bands. For example, the bandwidth available in the $70 \mathrm{GHz}$ and $80 \mathrm{GHz}$ bands, a total of $10 \mathrm{GHz}$, is more than the total sum of all other licensed spectrum available for wireless communication.

- Larger bandwidth channels are possible, of $2 \mathrm{GHz}, 4 \mathrm{GHz}, 10 \mathrm{GHz}$ or even $100 \mathrm{GHz}$, enabling fiber-like capacity.

- The spectrum can be made available quickly and can be reused easily with the limited propagation range in this band.

- Lower spectrum license costs lead to a lower total cost of ownership and lower cost per bit of radio systems using this spectrum. Besides, the cost of licensing E-band (60-90 GHz) links is exceptionally low, even below the thousands of euros per link for 10 years of interference protected use which is much lower price than in the microwave band [6].

Given the broad field of exploration and its capabilities, this frequency band is the one chosen in this thesis. Although a large part of the work is focused mostly on $30 \mathrm{GHz}$, there are also some antennas at $60 \mathrm{GHz}$. In order to understand the context in which these antennas are framed, and the challenges that have been faced, a general overview of the mm-wave band is described. A similar scheme of that well-suited in [7] is followed. There, historical background, propagation characteristics, key benefits, and applications are highlighted as the main points. Implicitly, concepts belonging to these four points are referenced somehow in every work in the literature. 


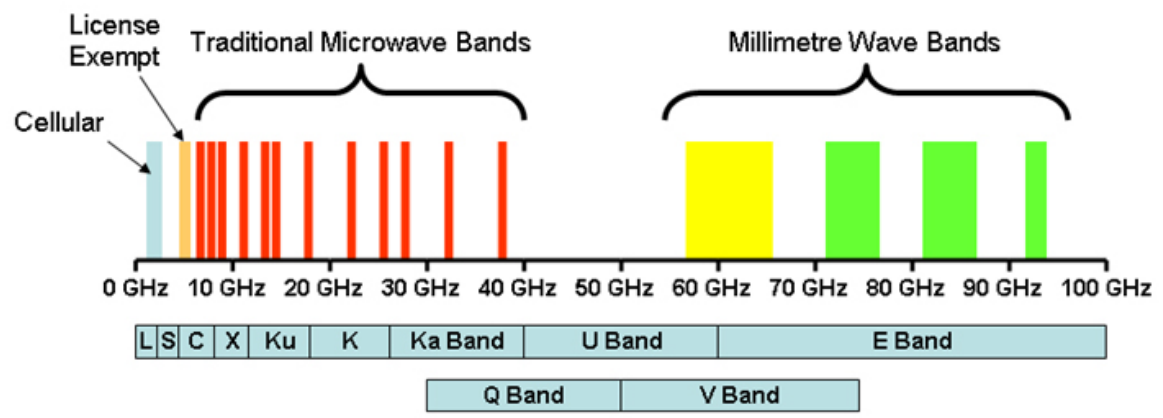

Figure 1.1: Millimeter wave spectrum [8].

\section{Historical Background}

The history of mm-waves starts only a few years after the discovery of electromagnetic waves by Heinrich Hertz and the first use of mm-waves is a direct consequence of the Hertz's work. Hertz did a series of experiments between 1886 and 1888 and stated that electromagnetic energy travels in a wave like motion, precisely similar to that of light and with velocity the same as that of light. He verified experimentally, for the first time, that the theoretical predictions of James Clerk Maxwell were correct. He also simplified Maxwell's mathematics and wrote down the set of equations which we today refer to as Maxwell's Equations. Hertz's pioneering series of discoveries was soon successfully repeated and extended by other scientists throughout the world. The theoretical basis of microwaves was also being pursued; notable is the first explanation of the modes of propagation in a waveguide by Lord Rayleigh in $1897[9]$.

Some researchers extended the operation frequency up into mm-wave band. Of particular interest is the careful and meticulous work of Professor Jagadis Chunder Bose of Presidency College, Calcutta, India. In 1897 he demonstrated a complete millimeter waveguide bench. Thus within a decade of the discovery of radio waves, microwaves and mm-waves had been established. With such a promising start, it might have been expected that microwave and mm-wave systems would have an assured future. However, this did not happen.

Following Bose's research, mm-wave technology remained within the confines of university and government laboratories for almost half a century. The technology started to see its early applications in Radio Astronomy in the 1960 's, followed by applications in the military in the 70's. In the 80's, the development of mm-wave integrated circuits created opportunities for mass manufacturing of mm-wave products for commercial applications. In the 1990's, 


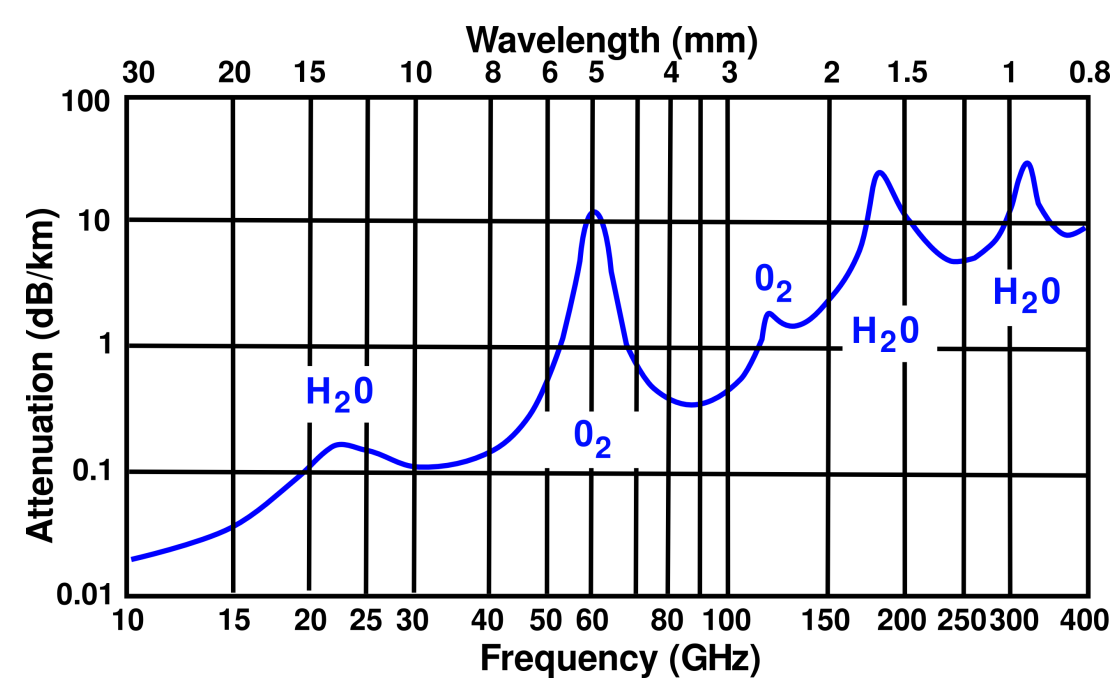

Figure 1.2: Expected atmospheric path loss as a function of frequency under normal atmospheric conditions [14].

the advent of automotive collision avoidance radar at $77 \mathrm{GHz}$ marked the first consumer-oriented use of millimeter wave frequencies above $40 \mathrm{GHz}$. In the 2000's the FCC authorized the use of 71-76 GHz and $81-86 \mathrm{GHz}$ for licensed point-to-point communication, creating a fertile ground for new industries developing products and services in this band. [7]. Finally, in the 2010s, several emerging $60 \mathrm{GHz}$ standards are targeted toward short-range wireless personal area networking (WPAN) such as high-definition streamed multimedia and high-speed kiosk data transfers [10]-[11]. Currently, the two most popular $60 \mathrm{GHz}$ standards, IEEE 802.15.3c and WirelessHD, will primarily provide $\mathrm{Gb} / \mathrm{s}$ streamed video and audio. Next-generation wireless local area networking (WLAN) will also exploit $60 \mathrm{GHz}$ spectrum through the development of the IEEE 802.11ad and WiGig standards [12]-[13].

\section{Propagation Characteristics}

Radio signals of all types, as they propagate through the atmosphere, are reduced in intensity due to the atmosphere effect. The atmospheric loss is defined in terms of decibels (dB) loss per kilometer of propagation (Fig. 1.2).

The propagation characteristics of mm-waves through the atmosphere depend primarily on atmospheric oxygen, humidity, fog and rain. The signal loss due to atmospheric oxygen, although a source of significant limitation in the 60 $\mathrm{GHz}$ band, is almost negligible in the 70 and $80 \mathrm{GHz}$ bands. Of all atmospheric 


\section{CHAPTER 1. INTRODUCTION}

conditions, rain causes the most significant loss of $70 \mathrm{GHz}$ and $80 \mathrm{GHz}$ signal strength, as is the case with microwave signals as well.

Consequently, $70 \mathrm{GHz}$ and $80 \mathrm{GHz}$ bands are projected for outdoor applications including mobile communications, Point-to-Point (P2P) high data-rate links, vehicular radars $(77 \mathrm{GHz})$, radiometry $(94 \mathrm{GHz})$, or imaging. On the other hand, since the frequencies surrounding $60 \mathrm{GHz}$ present high atmospheric attenuation, they are very suitable for a short-range scenario in which it can be taken advantage of the isolation from sources of interference. Consequently, there is a huge interest in these frequencies, generally for high-data rate wireless indoor communications.

It must be also indicated that higher altitudes in the atmosphere present higher attenuation. For common communication links or other terrestrial applications this fact is not a problem however it must be taken into account to plan satellite communications or atmospheric radiometry experiments [15].

\section{Applications}

Radar and imaging, indoor communications, outdoor communications, sensors, aerospace applications or satellite communications are highlights of a growing list of applications at mm-wave frequencies. These applications are causing excitement in engineering companies involved in mm-wave components, assemblies and test equipment. As development continues and costs come down, mm-waves will become a widely used portion of the electromagnetic spectrum. Among the applications mentioned, we will focus on satellite communications (SATCOM).

\subsection{SATCOM}

The field of SATCOM is undoubtedly a hot topic today. In recent years, the demand for satellite capacity has grown similarly to other wired and cellular wireless communications technologies, and the market trend is to increase the service throughput speeds in at least one order of magnitude compared to existing Ku-band systems. Demand for Ka-band satellite capacity is expected to grow over the next ten years. The Middle East, Asia, and Africa will be areas of high demand as a mix of communication applications including trunking and backhaul services, broadband access, enterprise networks and government communications demand more Ka-band capacity. As a revealing piece of information, the European Space Agency has funded more than 150 projects during the 2000s and 2010 for the development of applications in SATCOM within the ARTES program.

The primary objective of the thesis is the design of high-gain low-profile lowcost and low-loss antennas at millimeter-wave band. Gap Waveguide Antennas, 


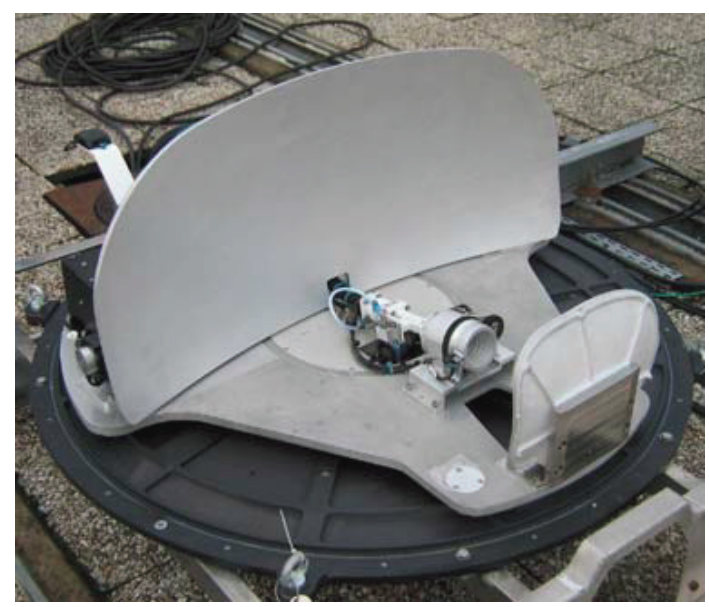

Figure 1.3: Ku-Band dual reflector antenna [16].

are intended to contribute one more step in the achievement of solutions with all the desired characteristics for SATCOM antennas. In this regard, satisfactory designs enjoying TX/RX operation, dually polarized, low profile and low cost antenna arrays for SATCOM on the move in Ka-band, supporting broadband applications, are scarce.

Nevertheless, it should be noted that efforts in this direction have already been made in the past using antennas such as parabolic reflectors, reflectarrays, lenses or waveguide arrays. These type of antennas are widely developed and studied, and they can meet some of the SATCOM requirements. The main characteristics of these contributions are briefly reviewed below. The literature on high-gain antennas at mm-wave band is very extensive, so the references provided are in some way related to the topics covered in this thesis.

\section{Parabolic Reflectors}

Reflector antennas with mechanical beam scanning provide high gain and acceptable side-lobe level with minimal price. An important advantage of such antennas is the possibility of combining TX and RX antennas in the same aperture as well as operating in multiple bands. The most used type of reflector antennas are dual-reflector antennas. On the other hand, a typical drawback of reflectors is that they are bulky and show a high profile. Therefore, the interest to find low-profile reflector antennas has grown in the last years. Many private companies such as ViaSat, Space Engineering, Thales Alenia Space or Hispasat, collaborate with public entities in the achievement of this objective. Some featured examples can be found in [16]-[18]. 


\section{CHAPTER 1. INTRODUCTION}
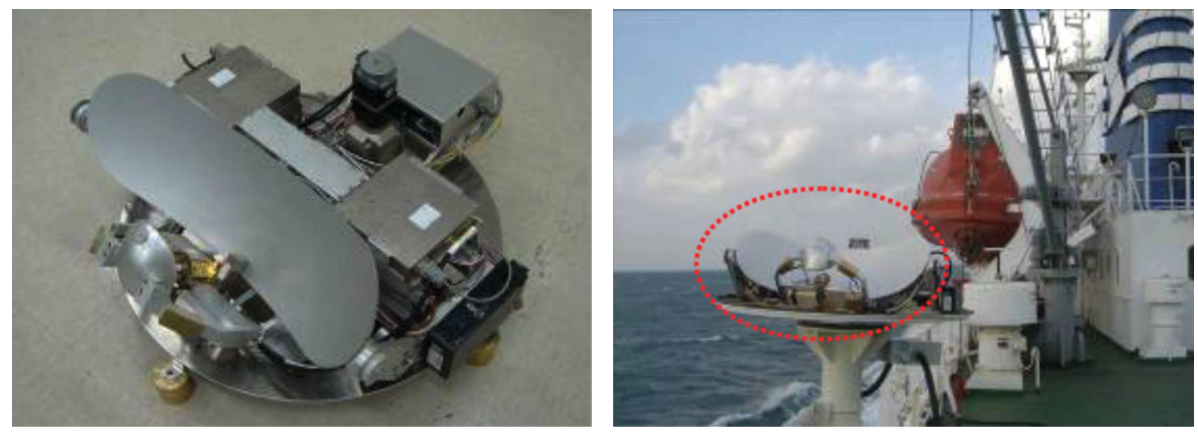

Figure 1.4: (Left) Photo of the entire assembled mobile antenna system and (right) antenna system installed on a large test ship [17].

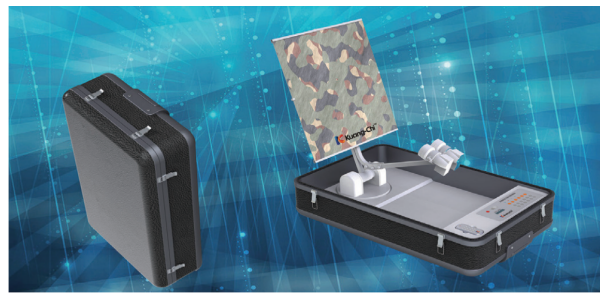

Figure 1.5: The Kuang-Chi flat-panel reflector antenna for Satcom, in a suitcase [18].

In [16], a dual offset Ku-band reflector antenna on board high-speed trains and ground vehicles was presented. In addition the $\mathrm{Ku}$-band mobile terminal evolution, in terms of performance improvement and possible upgrading to Ka band, was addressed. A photograph of this reflector is shown in Fig. 1.3.

Besides, in [17] a tri-band mobile antenna system which operates by utilizing the geo-stationary satellite Koreasat-3 in tri-band ( $\mathrm{Ka}, \mathrm{K}$, and $\mathrm{Ku}$ ) was designed, and a pilot product was fabricated and tested. This tri-band antenna system permits the provision of diverse broadband services including super high-speed interactive internet access, video communication, video on demand, and high quality satellite broadcasting. A detail image of the triband parabolic reflector and its placement on a ship is shown in Fig. 1.4. Furthermore, a recent contribution presented in [18] uses metamaterials to obtain a reflector with a planar shape that is only $2 \mathrm{~mm}$ thick. Fig. 1.5 shows an illustration of a portable metamaterial satcom reflector antenna in a suitcase.

In a different line of research, it appears the depoyable parabolic reflectors. This type of reflectors are useful to be embarked on satellites. One of the most recent contributions in the field of satellite applications is that made by 

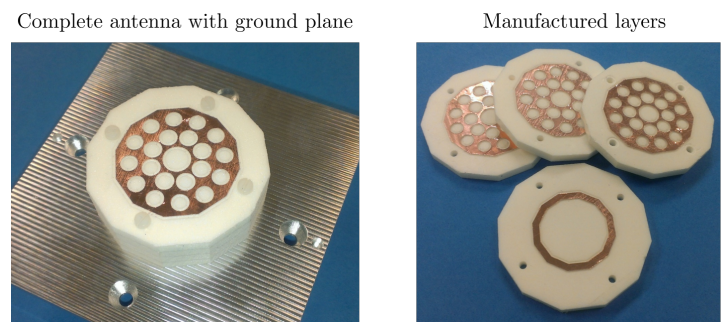

Lateral view

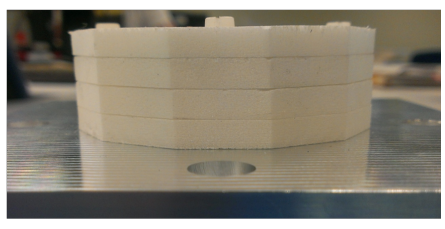

Figure 1.6: Example of planar lens in front of a square aperture on a ground plane presented in [22].

NASA's Jet Propulsion Laboratory and CalTech [19]. This paper introduces a $42.8 \mathrm{dBi}$ gain deployable Ka-band reflector antenna folding in a $1.5 \mathrm{U}$ stowage volume suitable for $3 \mathrm{U}$ and $6 \mathrm{U}$ class CubeSats. The deployable antenna is an unfurlable mesh reflector with 32 ribs, and it resembles an umbrella. A more general review of recent developments in advanced antennas for small satellites is presented in [20].

Furthermore, in the context of new manufacturing techniques, there are also studies in the line of lowering the weight of reflectors through additive manufacturing technology. In [21] a lightweight, cost-efficient, wideband, and high-gain 3D printed parabolic reflector antenna in Ka-band is presented. A $10 \lambda$ reflector is printed with a biodegradable type of plastic. The reflecting surface is made up of multiple stacked layers of copper tape, thick enough to function as a reflecting surface.

\section{Lenses}

The lens antenna is a 3-dimensional electromagnetic device which has a refractive index other than unity. It consists of electromagnetic lens along with the feed. These antennas are similar to glass lenses used in the optical domain. The major advantages of lens antennas are narrow beamwidth, high gain, and low sidelobes. On the other hand, the main drawback are the volume, the weight and in addition, the losses in the dielectric and the reflection in the discontinuities.

Many interesting examples of lenses in the mm-wave band can be found in [24]-[27]. Moreover, its usefulness and feasibility as a solution for SATCOM antennas is emphasized in recent contributions based on printed lenses as [28][32]. Printed lenses (Fig. 1.6) are more appropriate for mobile communications thanks to their lower weight and profile.

Also, there are numerous studies based on lenses that have demonstrated their viability as multi-beam systems such as the Luneburg lens [33]-[35]. Re- 


\section{CHAPTER 1. INTRODUCTION}
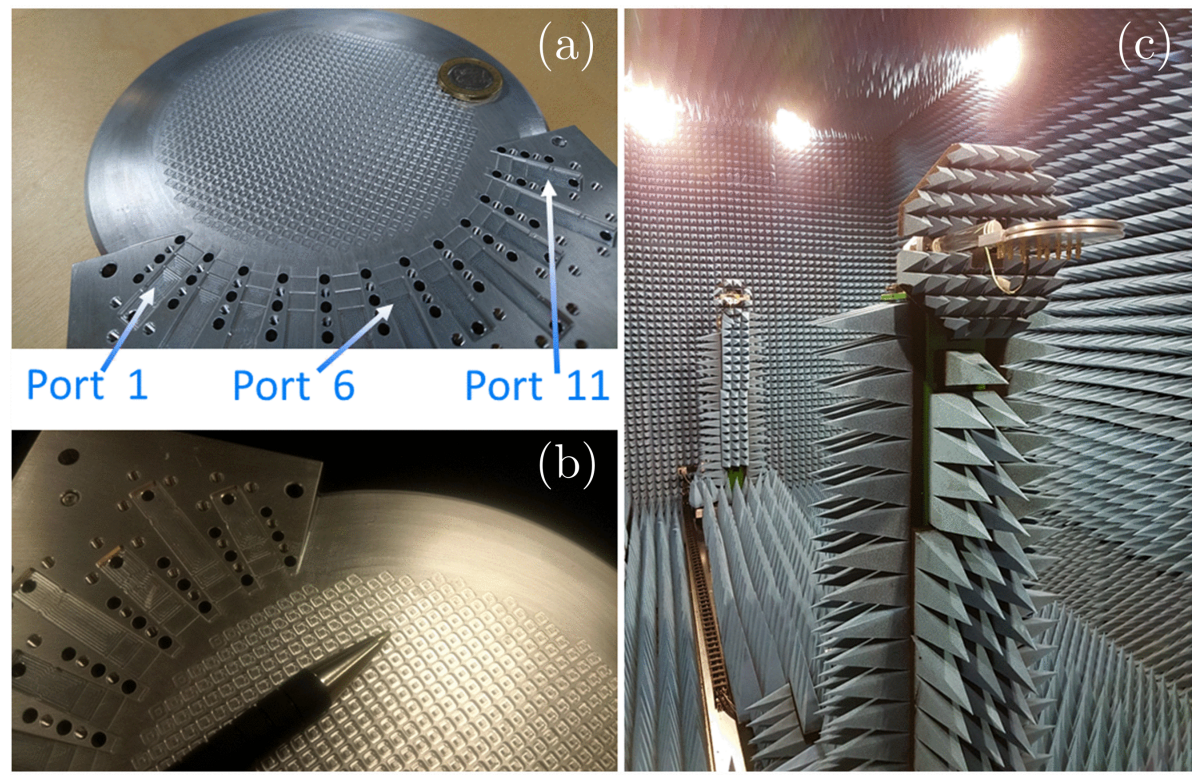

Figure 1.7: (a) Photograph of the bottom layer of the manufactured Luneburg lens with numbered ports. (b) Zoom of the lens and (c) measurement configuration at the anechoic chamber [23].

cent studies have been conducted using this type of lens since they are very attractive antennas for use in wide angle scanning applications. In this line of research, IETR researchers in Rennes proposed an all-metal Ku-band Luneburg lens based on variable parallel plate spacing Fakir bed (similar to Gap Waveguide technology) thus avoiding the use of dielectrics [36]. In addition, another completely metallic Luneburg lens (Fig. 1.7) was published making use of a glide-symmetric holey structure [23],[37].

\section{Reflectarrays}

Reflectarrays combine some of the advantages of reflectors and array antennas. A feed illuminates a usually planar reflecting surface with locally controlled surface impedance to produce a fixed or reconfigurable radiation pattern. From the reflectarrays it stands out its simplicity and low loss of the power distribution scheme. Besides, it is potentially associated with low cost and mass production of printed structures. Also, for large apertures, a reflectarray can be more accessible to manufacture and deploy than a large/shaped reflector. Main limitations are the narrow bandwidth, as demonstrated in [38] and [39], 


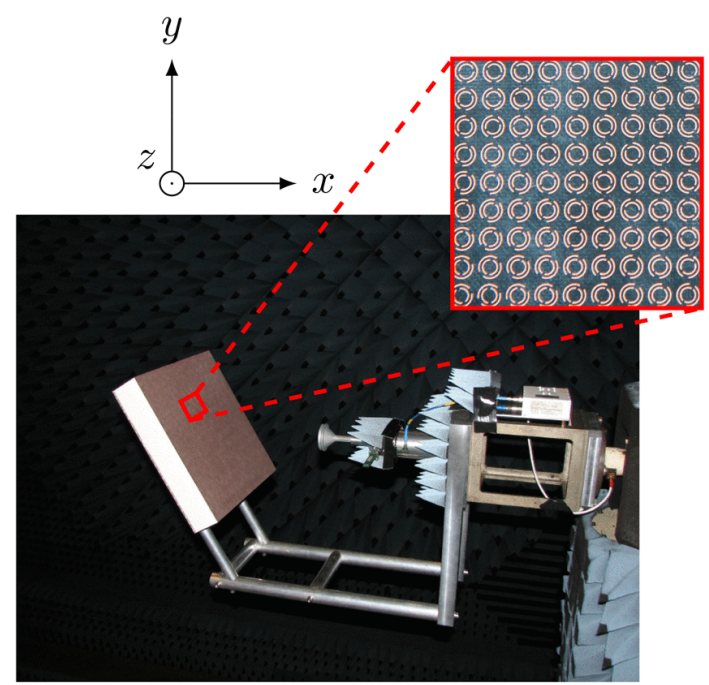

Figure 1.8: Offset reflectarray antenna in the DTU-ESA Spherical Near-Field Antenna Test Facility [40].

as well as the uncontrolled reflection of some of the incident power. Furthermore, the need of a primary feeder makes the whole structure bulkier than other solutions, such as planar arrays.

Nonetheless, many efforts are being made to reverse this situation. For example, in large reflectarrays, frequency bandwidth can be improved by stacking several layers of printed elements. The contributions made by Encinar in this regard are remarkable [41]-[45]. Other works also follow the same line of research. In [46], a novel dual-polarized stacked patch antenna element for wide-angle scanning is presented. Moreover, a $20 / 30-\mathrm{GHz}$ dual-band circularly polarized reflectarray antenna was introduced in [40] (see Fig. 1.8). Both contributions are aimed to be used for SATCOM on-the-move applications at Ka-band.

In addition, new possibilities are being explored. In [47] the recent developments in Liquid Crystal-based reflectarray antennas for mm-wave applications were presented. The authors proposed a reconfigurable antenna that can be manufactured without the need for integrating hundreds or thousands of devices in the array. Thus, the fabrication process is significantly simplified, which is especially beneficial for large reconfigurable arrays such as those required for SATCOM applications. Following with the reflectarrays focused on SATCOM on the move, it is interesting the recent work of Gao in [48]. There, a Ka-band low-cost beam-scanning folded reflectarray and a Ku-band low-cost 
dual-polarized reconfigurable reflectarray with single bit phase control by using PIN diodes, are presented. Both prototypes are examples of high-performance mm-wave smart antennas with reasonable cost.

\section{Antenna Arrays}

Antenna arrays is another alternative. However, there are scarce published works of high-gain low-profile full-metal structures focused on SATCOM applications in the Ka-band. Below Ka-band, printed solutions are the most widely used since microstrip technology allows easy integration with active components and takes advantage of the low costs of printed circuit technologies. Therefore, microstrip antennas have been widely used as a solution for compact high-gain arrays in the microwave band [49]-[50]. Years ago, attempts to use this type of antennas at frequencies higher than microwaves ( $\mathrm{K}$ band and above) were not satisfactory since the efficiency achieved was far back to compete with that of completely metallic structures [51].

Today, by combining metal feed networks with patches [52]-[53], a tradeoff between compactness and losses can be achieved although the full-metallic structures are more efficient (80-90\% instead of 50\%). Already in the mid-90s, the antenna group at Tokyo University of Technology proposed the use of fullmetal antennas instead of printed solutions. In their work presented in [54], it is defended that in printed antennas the loss becomes significant in the mm-wave band and only antennas with lower gain (20 to $30 \mathrm{dBi}$ ) or lower efficiency (20 to $40 \%$ ) had been reported until that day. Then, they proposed waveguide slot array antennas as an attractive candidate for high-efficiency planar antennas. However, the problem lies in the complex feeding structures they need. It is necessary to simplify these structures.

During the last 15 years, there is a boom in the research about full-metal antenna arrays in the mm-wave band. The objective is to combine all the advantages of the antennas previously proposed (parabolic reflectors, lenses, reflectarrays) and also to provide low profile, low weight, and mass production. We will focus on these approaches on the following. For an easier understanding, the antennas are classified taking into account their polarization type (linear, circular, dual-pol), their bandwidth characteristics (single or dual-band) or their steering capability (mechanical, hybrid or electronic).

\section{Linear-Polarized Antenna Arrays}

Following with the work developed at Tokyo Tech, the solution in [54] was a first approach. A straightforward design in the $\mathrm{K}$ band $(22 \mathrm{GHz})$ with a series feeding network with narrow frequency bandwidth was presented. This same group of researchers were developing during the second part of the $90 \mathrm{~s}$ continuous improvements of that idea in [55] and [56]. 

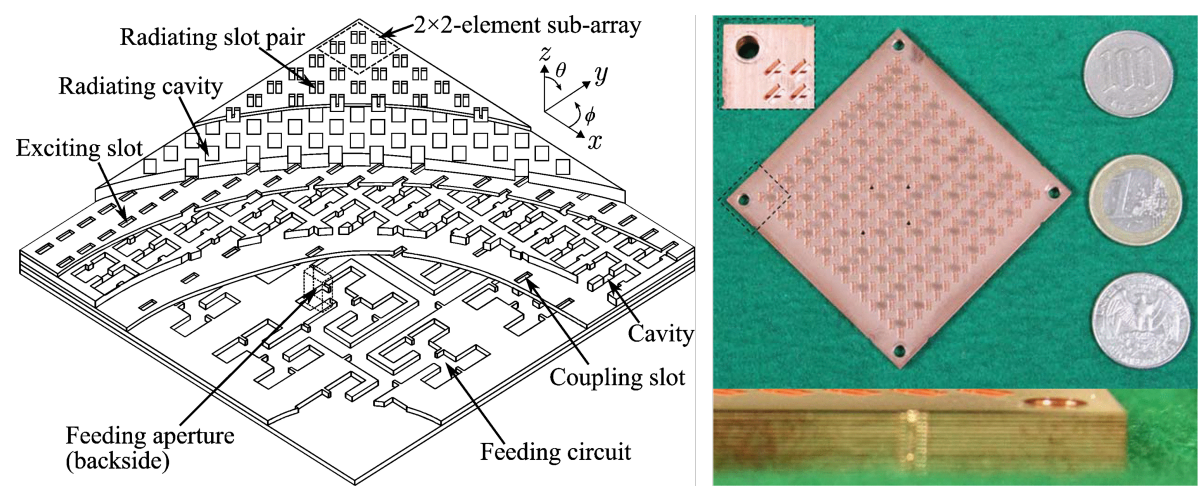

Figure 1.9: (Left) Configuration of the $16 \times 16$-element array. (Right) Photo of the fabricated E-band antenna using diffusion bonding introduced in [61].

Recently, during the 2000s and to date, the same research group headed by professors M. Ando and J. Hirokawa are keeping and improving the concept. Among the most notable works published recently, [57]-[63] stand out. All these fully metallic linearly-polarized antennas, share a common manufacturing technique known as Diffusion Bonding. An example of these antennas is shown in Fig. 1.9.

Diffusion bonding is a solid-state welding technique used in metalworking, capable of joining similar and dissimilar metals. The diffusion bonding at high temperature (about 1000 degrees) gives perfect electrical contacts among the laminated metal plates. According to Hirokawa [64]-[65], this fabrication technique has the potential of being low cost in the millimeter-wave band because of high-precision (about $20 \mu \mathrm{m}$ ) etching of metal plates. By contrast, another group working on high-gain, low-profile and low-loss antennas is the one of Chalmers University of Technology in Sweden. They claim the opposite, i.e., that diffusion bonding is an expensive technique [66]-[67]. This group, led by Kildal, pioneered the use of Gap Waveguide technology for the realization of fully metallic antennas in the millimeter-wave band. Practically all their proposals consist of multilayer linearly-polarized antennas composed by several unconnected layers, thanks to the properties of the Gap Waveguide technology. Notable contributions for their simplicity and performance can be found in [68]-[67]. There are much more examples of high-gain linear-polarized antennas in the mm-wave band [76]-[79], but they are not completely metallic since they make use of SIW or LTCC. Thereupon, they are out of the scope of this thesis. 


\section{CHAPTER 1. INTRODUCTION}
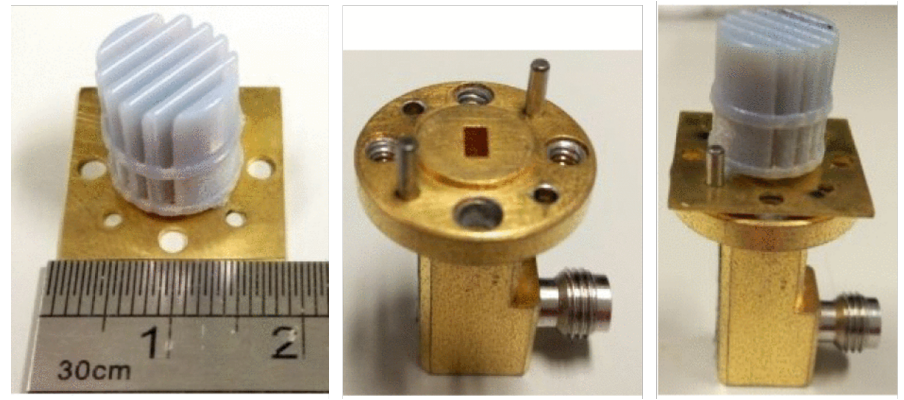

Figure 1.10: Photo of the fabricated polarizer antenna in [81].

\section{Circularly-Polarized Antenna Arrays}

The number of publications and research about full-metal antennas in the mm-wave band with circular polarization is significantly lower than the linearpolarized ones. Hardly any prototypes with these characteristics can be found and even less in Gap Waveguide technology. We highlight some outstanding publications in printed solutions.

A Ka-band circularly polarized (CP) planar array antenna with an axial ratio (AR) bandwidth of $14 \%$ and efficiency between 60 to $80 \%$ is presented in [80]. There, a stripline network and a waveguide transition are integrated into a $4 \times 4$ array. While it is an interesting antenna, the efficiency would be drastically reduced in a larger array. Another example can be found in [81]. This paper presents a wideband CP mm-wave band antenna by using a 3-D printed polarizer. The polarizer consists of several gaps and dielectric slabs which transform the polarization from linear to circular (Fig. 1.10). This solution, however, does not seem easily extendable to a larger array and the dielectric polarizer may lead to higher losses.

Regarding GW arrays, a few examples can be found. In [82] a $1 \times 8$ array was presented. However the authors recognized that further investigations must be carried out to extend it to a two-dimensional array since the proposed RGW network, in this case, is unable to achieve the desired arrangement. This antenna is not completely metallic either, since it makes use of PCB elements.

Another proposal, very similar to the previous one, but fully metallic is shown in [83]. This paper presents a novel $77 \mathrm{GHz}$ slot array antenna using an RGW as feeding network. The all-metal structure consists of two layers, being a promising planar geometry without dielectric losses. However, the proposed architecture will not be easy to be extended to a larger array.

Very recently, a full-metal $2 \times 2$ slot antenna array fed by ridge gap waveguide (RGW) technology for Ka-band was described in [84]. The proposed 
antenna array consists of two parallel plates, including radiating layer and feeding network in the bottom and it shows a $3-\mathrm{dB}$ axial ratio (AR) bandwidth of $11.3 \%$. However, in this case, only simulations were presented.

Furthermore, one of the most recent contributions using diffusion bonding is presented in [85]. The antenna consists of an array of $16 \times 16$ circularly-polarized radiating elements with a double-layer corporate feeding network (Fig. 1.11). The authors claim that diffusion bonding seems nowadays to be the only manufacturing technique capable of implementing waveguide fully metallic antenna arrays operating at W-band with gains over $20 \mathrm{dBi}$ and efficiencies over 50 percent.

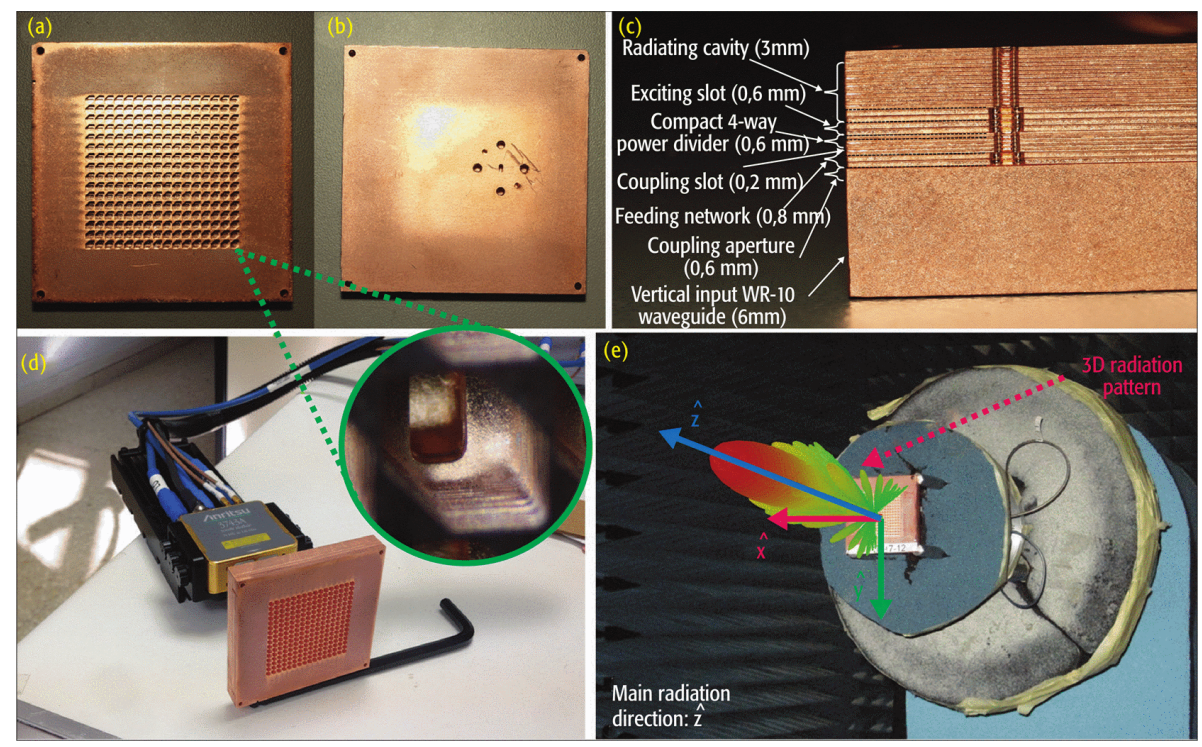

Figure 1.11: Photography of the diffusion-bonded antenna in [85]: (a) The top view; (b) The bottom view; (c) The side view; (d) Reflected power measurement and (e) Radiation measurement in an anechoic chamber.

\section{Dual-Polarized Antenna Arrays}

Again, we are facing a type of antenna of potential interest for SATCOM. It is easy to find dual-polarized printed antennas [86]-[87]. In addition, horn arrays are good candidates to satisfy the current need for medium-profile antennas for mobile user terminals for SATCOM applications [88]. Notwithstanding, there are not many examples of metallic low-profile antennas at frequencies beyond $30 \mathrm{GHz}$. 


\section{CHAPTER 1. INTRODUCTION}

Nevertheless, in the $\mathrm{X}$ band, there are three contributions worthy of mention [89]-[91]. Among these three dual-polarized antennas in the X-band, the contribution made in [91] is highlighted. It includes additive manufacturing (Metal Laser Sintering) as the fabrication technique. That prototype provides not only a high gain and planar profile but it is also low-cost and light weight.

Undoubtedly, one of the most recent and interesting contributions in this field is the dual-polarized antenna presented in [92] (Fig. 1.12), again developed by the team of Ando and Hirokawa. They proposed a $16 \times 16$-element array fabricated by diffusion bonding. A thick cavity structure and dense element spacing is designed to suppress the grating lobes. The antenna efficiency is near $80.0 \%$ over 60 to $64 \mathrm{GHz}$ for two different polarizations. Good polarization purity is also achieved since the decoupling between both input ports is larger than $50 \mathrm{~dB}$. Perhaps the biggest drawback of the antenna is precisely the technique used. Although the excellent performance of diffusion bonding is out of doubt, it is still an alternative more expensive than other techniques such as milling. However, the precision of milling at $60 \mathrm{GHz}$ is certainly critical.
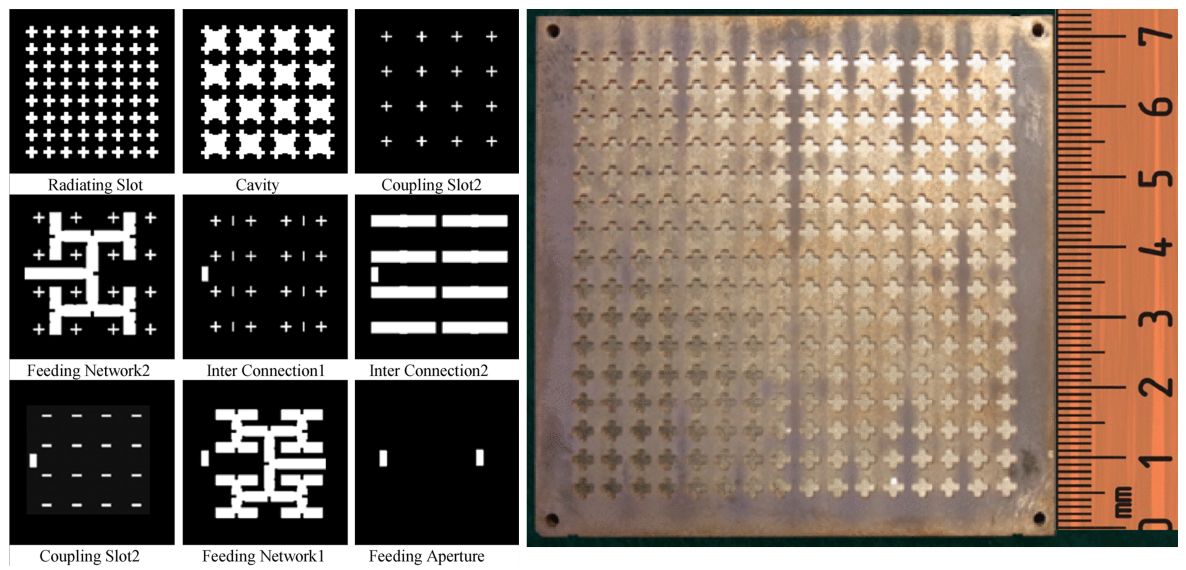

Figure 1.12: (Left) Cut-plane patterns for each layer for $8 \times 8$-elements in the xy-plane and (right) the fabricated $16 \times 16$-element array antenna for dualpolarization presented in [92].

\section{Multi-Beam and Beam-Steering Arrays}

One of the most researched topics at present is the possibility of steering the beam or synthesizing multiple beams. Therefore, in addition to all the aforementioned properties that arrays should have (high gain, high reliability, small size and weight, low profile, acceptable price, etc.) we must add the possi- 
bility of being able to steer the beam. The capability to steer the beam is necessary for mobile systems such as SOTM. Traditional approaches for beam steering are based on mechanical steering, hybrid solution (electro-mechanical) or electronic steering.

There are very interesting examples of multibeam antennas. In [93], a novel multibeam V-band antenna based on a Butler matrix with sidelobe level (SLL) control is proposed. Also, a novel dual-layer multibeam pillbox antenna with a slotted waveguide radiating part in substrate-integrated waveguide in [94] (Fig. 1.13) and a multibeam antenna system fed by a compact Rotman lens in [95] are proposed.

Regarding beam-steering arrays, two-dimensional mechanical scanning is the most usual option today. An attractive hybrid solution is to feed a reflector or a lens at its focal point, which can be displaced slightly, producing the desired steer of the beam. Examples of this type of solution are presented in [96]-[100]. Another versatile solution, very recently published but of greater complexity, is to rotate mechanically each of the elements of the array [101]. In addition, in [102] a new continuous beam steering antenna in gap waveguide technology for $60 \mathrm{GHz}$ applications is presented (Fig. 1.14). The antenna is flat, has a wideband characteristic, broad coverage and does not require phase shifters for beam steering capability.

A more evolved solution is to combine mechanical with electronic scanning, allowing a compromise between antenna price and speed of beam steering [103]-[109]. In general terms, the combination of mechanical and electronic steering allows to implement antennas with lower profile, very simple mechanical structure based on a single axis of rotation and a reduced number of active components.

The most desired solution, however, is the two-dimensional electronic scanning. This option provides fast-tracking but requires a large number of costly phase-shifters. To satisfy the gain requirements, power amplifiers in the TX antenna and LNAs in the RX antenna are needed. Satisfactory examples of antennas with these characteristics have been demonstrated in the last five years [110]-[115].

In any case, some issues such as good antenna performance in terms of gain, side lobe level, beam steering range, as well as the compliance with international regulations have not been fully resolved yet. Besides, the high cost of manufacturing limits their ability to sell them massively in the mobile communications market (ships, cars, trains, aircraft). For these reasons, there is an enormous interest both in public agencies (NASA, ESA) and private companies (Phasor, Kymeta) in the development of new disruptive concepts to bring down cost significantly with respect to current solutions. 


\section{CHAPTER 1. INTRODUCTION}

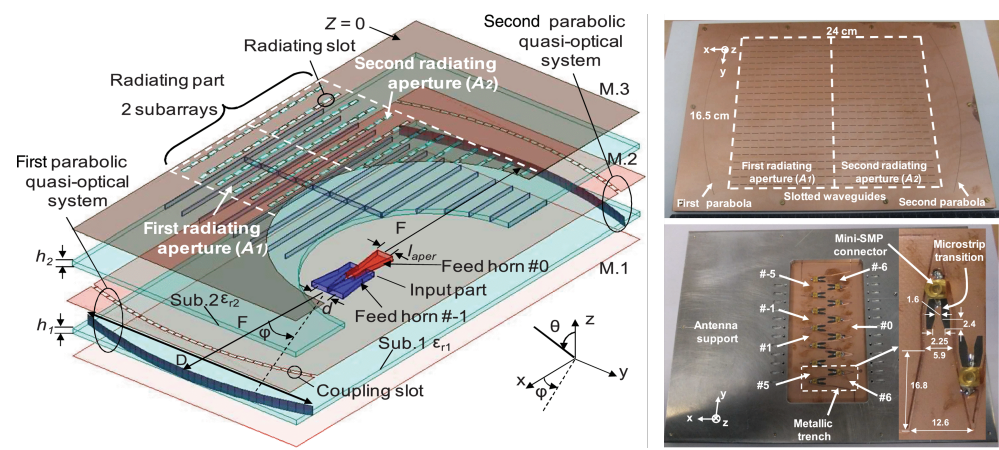

Figure 1.13: (Left) Pillbox antenna structure with two radiating apertures and (Right) top and bottom view of the antenna presented in [94].
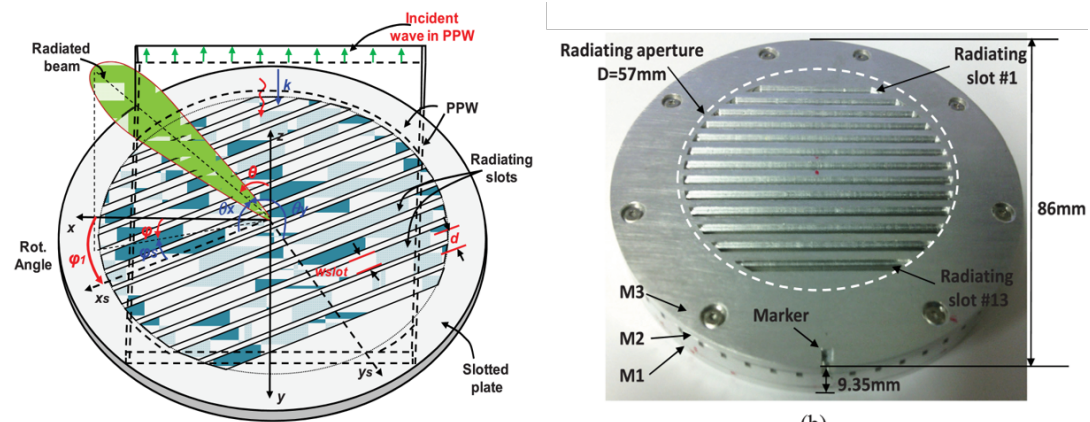

(b)

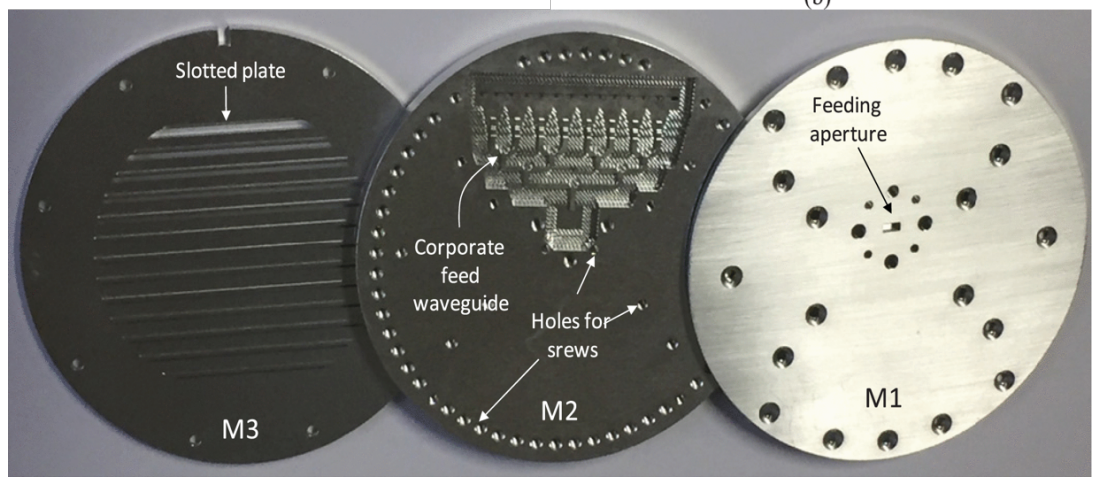

Figure 1.14: Simplified model showing the scanning mechanism of the proposed concept and the manufactured antenna prototype of [102]. 


\subsubsection{Gap Waveguide Technology}

Gap Waveguide technology has been the result of joint work between researchers from different countries, whose collaboration and common interest managed to develop a technology that today has reached its maturity, endorsed by the large number of papers that arise each year. It can be considered today a mature technology that can serve to respond to some problems arising in the mm-wave band, some of them already exposed in the previous section. The germ of Gap Waveguide Technology can be traced back to 1990 .

Per-Simon Kildal, from the Chalmers University in Sweden, took the Soft and Hard surface concept from acoustics and he used it in electromagnetics [116]. The classical way to realize a soft surface is to corrugate an ideal conductor with transverse rectangular grooves. The performance of such corrugations is well known. In principle, they act as shorted transmission lines for the longitudinal polarization and transform the short to $Z_{t}=\infty$ at the aperture of the corrugations for a proper groove depth $d$. On the other hand, the hard surface can be realized by corrugating an ideal conductor with longitudinal grooves and filling them with a dielectric material. These corrugations act like shorted transmission lines for the transverse polarization, and the depth of them must be chosen to give $Z_{t}=\infty$ at the aperture of the corrugations [117]. So, the soft surface can stop waves of any polarization propagating along the surface, and the hard surface can, on the other hand, enhance propagation along the corrugations [118]. This concept was then used for removing blockage by cylindrical objects [119]. This publication dates to 1996, then ten years later, in 2006, such blockage reduction became a popular research topic known under the name cloaking [120]-[121].

Taking another step in this direction, the need to include some guiding structure in the parallel plate waveguide to create a stable plane wave was intuited by Padilla et al. [122]. They proposed to arrange a set of parallel strips, alternating between the perfect electric conductor (PEC) and artificial magnetic conductor (AMC), simultaneously on the lower and upper plates, to create what they called virtual TEM waveguides. However, the intended effect was not entirely achieved.

However, the work in [122], was in the right direction and, in 2007, it inspired researchers of the Polytechnic University of Valencia in Spain (UPV) on the proper selection of the boundary conditions to create local quasi-TEM modes [124]. It turned out that in a parallel-plate waveguide formed out of a PEC plate and a hard surface [116], field propagates in the so-called hard direction, i.e., along the metallic grooves of the corrugated surface, while it experiences a strong transverse attenuation. The key ingredient, though, for this effect to be achieved is that waveguide height be less than quarter wavelength. 


\section{CHAPTER 1. INTRODUCTION}

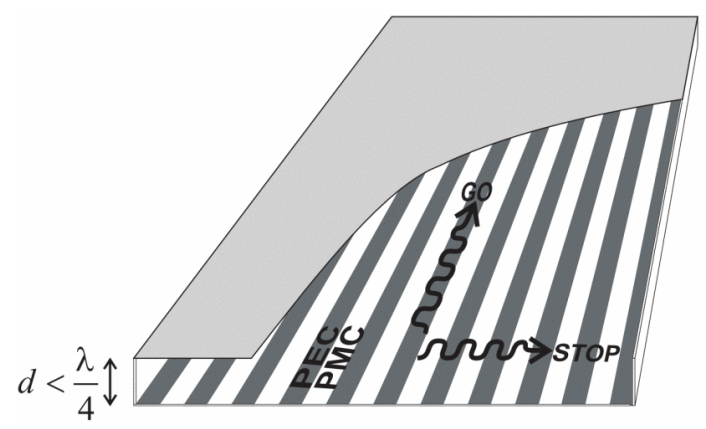

Figure 1.15: PEC/PMC interpretation of a corrugated surface and its overall effect on the waves in a parallel plate waveguide [123].

The use of a hard surface as a wave-guiding device motivated researchers at UPV to seek collaboration from Prof. Kildal, to further understand its working principle. Kildal became interested in the subject and teamwork was initiated.

Ideally, a hard surface can be seen as a PEC/PMC strip grid, i.e., a grid of parallel strips, where every second strip is a $\mathrm{PEC}$ and a perfect magnetic conductor (PMC), respectively [125]. The PMC strips can be realized as dielectrically filled metal grooves with an effective quarter wavelength depth. A quite simple analysis shows that the combination of a PEC on the upper plate and a PMC strip on the lower one creates a cutoff condition, provided that the gap between them is less than a quarter wavelength. Therefore, after crossing a few of these PEC/PMC areas in the transverse direction, the wave is profoundly attenuated, producing a local confined wave propagating only in the longitudinal direction as depicted in Fig. 1.15.

In 2009, Kildal was able to give the original idea a final twist by conceiving a cutoff condition which, unlike the hard surface, was independent of the chosen direction of propagation. For that purpose, a high-impedance surface would replace the corrugated surface. In such environment, a completely arbitrary propagation path can be opened in the heart of the high-impedance surface just by placing a ridge, digging up a groove or even laying down a thin dielectric layer with a microstrip on it. This resulted, finally, in the patent of the idea that year [126]. Commonly, the high-impedance condition is achieved through a bed of nails which is $\lambda_{0} / 4$ in height at the center frequency. Around an octave bandwidth can be achieved this way. Nevertheless, other periodic details, such as mushroom-type can be used as well [127].

After this new finding, Professor Kildal proposed to professor Alejandro Valero-Nogueira from the Polytechnic University of Valencia to continue the teamwork with Chalmers University and E. Alfonso brought forth the first Ph.D. thesis on Gap Waveguides in 2011 [128]. After this first thesis, several 

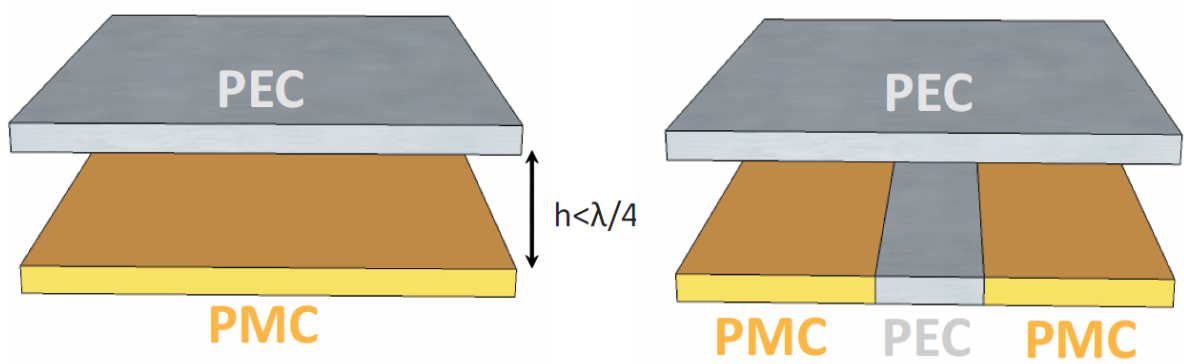

(a)

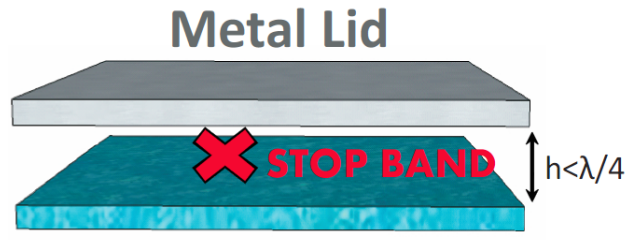

AMC

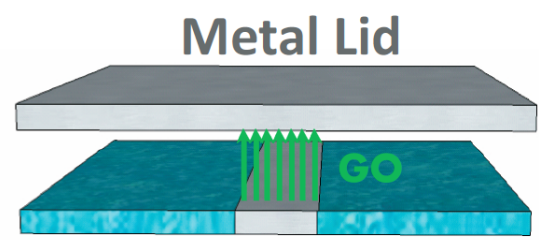

AMC Strip AMC

(b)

Figure 1.16: (a) Basic principle of operation of gap waveguides with ideal PEC-PMC parallel-plates (left) and PEC-PEC parallel plates, one of which is embedded by a PMC on both sides (right). (b) Realization of the same concept with a real metal lid and a AMC surface around the strip [136].

doctoral students have focused their research on this topic; the present thesis an example that the subject is open for new contributions.

That first thesis on gap waveguides was aimed at introducing them as a promising waveguide technology for high-frequency applications, as well as to verify its performance through the realization of circuits [129]. For circuit design it is essential to characterize the waveguide as a transmission line, or in other words, to know the propagation constant and characteristic impedance. The initial theoretical and numerical studies on the propagation characteristics of gap waveguides [130], [131], [132], [133] set up the basis for a further development of the technology with the design and experimental demonstration of simple microwave passive circuits, such as power dividers and couplers [134], at the beginning, and more complicated ones, like filters and corporate feeding networks for antennas later on [135]. 


\section{CHAPTER 1. INTRODUCTION}

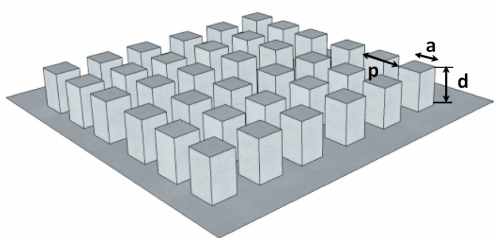

(a)

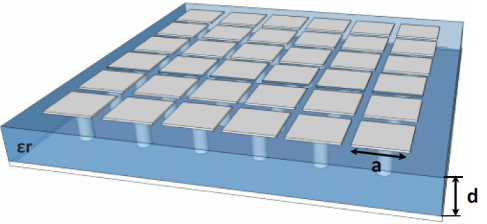

(b)

Figure 1.17: (a) Bed of nails. Common configuration of Gap Waveguide Technology. (b) Mushroom EBG configuration [136].

\section{Principle of operation of gap waveguides}

The fundamental principle of operation of Gap Waveguide Technology is illustrated in Fig. 1.16. Ideally, it consists in two parallel plates, one PEC plate above a PMC plate (Fig. 1.16a). So, if the distance between the plates is smaller than a quarter wavelength, then no wave is allowed to propagate since all parallel-plate modes will be in cut-off, due to the boundary conditions on both surfaces. Now, if the lower plate is replaced and PEC is added, the conditions change. Accordingly, it is well known that parallel-plate TEM waves will propagate in the gap between them. If the bottom PEC plate is surrounded by a PMC plate on both sides, as shown in the illustration on the right of Fig. 1.16a, then there will be no waves propagating in the gap between the PEC-PMC plates, confining only a local TEM mode propagating in the center, in the gap between both PEC layers, thus creating the gap waveguide [137].

The PEC plate is easily realized, but PMCs are not available in nature. Thus, in practical applications, the PMC condition is emulated by an artificial magnetic conductor (AMC) in the form of periodic textured structures such as nails or mushroom structures [118]. Thus, a gap waveguide can be realized with a groove, a ridge or even a metal strip, embedded in a textured surface which provides the high impedance, allowing only a local mode to propagate along the metal path, as shown in Fig. 1.16b.

The most common high-impedance surface used in gap waveguides are a textured surface made of periodic metal pins, also called a bed of nails [138] (Fig. 1.17a), and, to a lesser extent, mushroom-type EBG textures, as proposed in [118] (Fig. 1.17b). The characteristics of the parallel-plate stopband provided by these structures are presented in [127]. The bed of nails can be easily realized with milling techniques or 3D printing, and it is entirely made of metal, being suitable for high-frequency applications. The nails (or pins) work as a high impedance surface within a stopband (or bandgap) defined by a lower and an upper cut-off frequency. The height of the pins must be approximately $\lambda / 4$, 


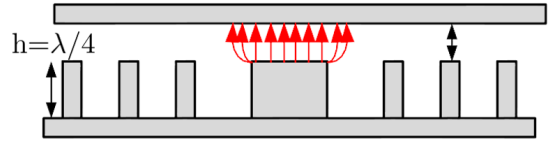

Q-TEM mode in Ridge Gap Waveguide

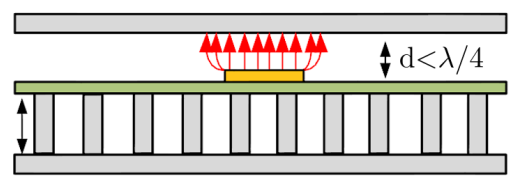

Q-TEM mode in Inverted- $\mu$ strip Gap Waveguide

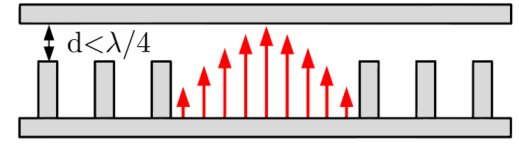

$\mathrm{TE}_{10}$ mode in Groove Gap Waveguide

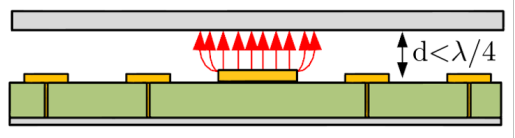

Q-TEM mode in $\mu$ strip Ridge Gap Waveguide

Figure 1.18: Different Gap Waveguide geometries and their desired propagation modes [139].

to transform a short circuit (PEC) to an open circuit (PMC) and the period $(p)$ must be less than half wavelength. The lower cut-off frequency is then determined by the height of the nails, while the upper cut-off limit is limited by the frequency at which $d+h=\lambda / 2$, where $h$ is the height of the air gap, which must be smaller than $\lambda / 4$.

Once this textured surface made of metal nails is created, the desired stopband within a specific range of frequencies is achieved. Since the given structure does not allow propagation inside that stopband, some modifications must be applied in order to create waveguiding channels. The way in which these modifications are done, leads to different Gap Waveguide types, described next.

Groove Gap Waveguide (GGW), Ridge Gap Waveguide (RGW), Invertedmicrostrip gap waveguide (IMGW), Microstrip Gap Waveguide $(\mu \mathrm{GW})$ are the most developed nowadays (Fig. 1.18). In this work, the first two types (GGW and RGW) have been used.

\section{Losses}

In the beginnings of Gap Waveguide technology, it was necessary to demonstrate the behavior of one of the fundamental parameters of any guided structure, the losses. For this reason, a deep study of losses in Gap Waveguide structures was made in a broader way by Zaman [139] and more focused on microstrip GW by Pucci [140]-[144].

Since there are different types of Gap Waveguides, each of them has been studied separately in order assess its performance and make a comparison be- 


\section{CHAPTER 1. INTRODUCTION}

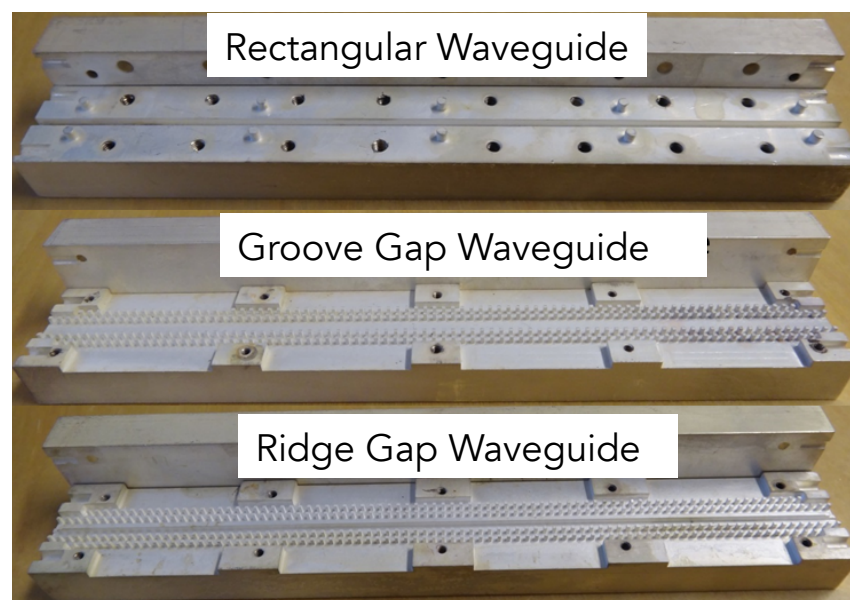

Figure 1.19: Prototypes for the experimental characterization of losses (RW, GGW and RGW) [2].

tween them. Throughout this section, the losses in each of the structures are briefly shown.

In [139], several gap waveguide models were manufactured and directly measured with a conventional network analyzer (VNA) to determine the attenuation and losses directly from the measurement of the transmission coefficient $S_{21}$. Several manufactured gap waveguide sections were used for that loss study. The guides that were used are shown in Fig. 1.19. For the sake of comparison, traditional technologies such as rectangular waveguide (RW), Substrate Integrated Waveguide (SIW) and Microstrip lines were also included in that study. The measured S-parameters for groove gap waveguide, ridge gap waveguide and the reference rectangular waveguide, are presented in Fig. 1.20. GGW and RGW present low reflection over the entire V-band: below $-20 \mathrm{~dB}$ for the groove gap waveguide and below $-17 \mathrm{~dB}$ for the ridge gap waveguide.

Ohmic loss for all the measured lines, not only those in the example of Fig. 1.20 but also other realizations of gap waveguide, are summarized in Table 1.1. On the one hand, Groove Gap Waveguide and Ridge Gap Waveguide were compared with a standard Rectangular Waveguide. On the other hand, the losses of the microstrip-ridge gap and inverted microstrip gap waveguide were compared with losses of the two open mm-wave microstrip lines presented in [145] and [146].

From the table, it is clear that the losses in the groove gap are comparable to the losses of a rectangular waveguide. The ridge gap waveguide has noticeably more losses than the rectangular waveguide because the guiding ridge is 
Table 1.1: Losses for the different versions of gap waveguide in the V-band [2].

\begin{tabular}{|c|c|c|}
\hline $\begin{array}{c}\text { Prototype } \\
\text { (frequency) }\end{array}$ & $\begin{array}{c}\text { Simulated loss } \\
(\mathbf{d B} / \mathbf{c m})\end{array}$ & $\begin{array}{c}\text { Measured } \\
\text { min-max loss } \\
(\mathbf{d B} / \mathbf{c m})\end{array}$ \\
\hline $\begin{array}{c}\text { Rect. Waveguide } \\
\text { (50 - 75 GHz) }\end{array}$ & 0.0136 & $0.0295-0.042$ \\
\hline $\begin{array}{c}\text { Groove gap } \\
\text { (50 - 75 GHz) }\end{array}$ & 0.019 & $0.03-0.0442$ \\
\hline $\begin{array}{c}\text { Ridge gap } \\
\text { (50 - 75 GHz) }\end{array}$ & 0.0373 & $0.058-0.0705$ \\
\hline $\begin{array}{c}\text { Microstrip-ridge gap } \\
\text { (56 - 68 GHz) }\end{array}$ & 0.0805 & $0.162-0.23$ \\
\hline $\begin{array}{c}\text { Invert-micro gap } \\
\text { (56 - 72 GHz) }\end{array}$ & 0.0934 & $0.21-0.288$ \\
\hline $\begin{array}{c}\text { Microstrip line } \\
\text { (50 - 75 GHz) }\end{array}$ & LCP : 0.23 & $0.62-0.77$ \\
\hline $\mathbf{0 . 1 2 7 - 0 . 2 m m ~ s u b s t r a t e ~}$ & Microstrip line \\
$\begin{array}{c}\text { (50 - 75 GHz) } \\
\mathbf{0 . 1 2 7 - 0 . 2 m m ~ s u b s t r a t e}\end{array}$ & Rogers $4003: 0.271$ & 0.7055 \\
\hline $\begin{array}{c}\text { Air filled SIW } \\
\text { (50 GHz) }\end{array}$ & 0.0621 & 0.0615 \\
\hline $\begin{array}{c}\text { Rogers-5880, SIW } \\
\text { (50 GHz) }\end{array}$ & 0.1327 & 0.2172 \\
\hline
\end{tabular}

narrower than the waveguide width. As a result, the current density is more significant over the ridge section.

Regarding the microstrip-ridge gap and inverted microstrip ridge gap waveguides, they are lossy, but both these versions of gap waveguide still outperform regular microstrip lines working at $60 \mathrm{GHz}$ frequency range as it was demonstrated in [147].

In [141], an analysis was made focused on the Microstrip Ridge Gap Waveguide looking for a low-cost and efficient solution for printed slot array antennas. It stands out in this work that grounding the ridge strip with vias is essential to reduce losses. Otherwise, EM fields extend under the strip, within the substrate, and spread more laterally, hence increasing both ohmic and dielectric losses. 


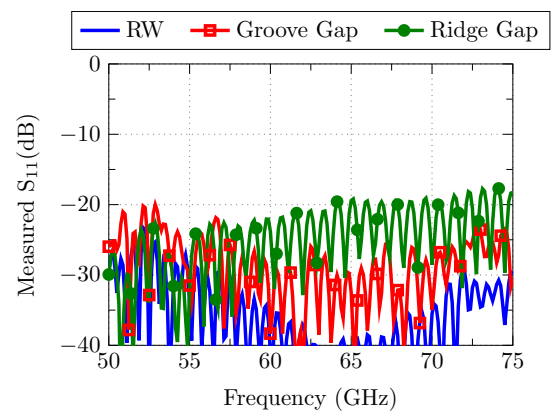

(a)

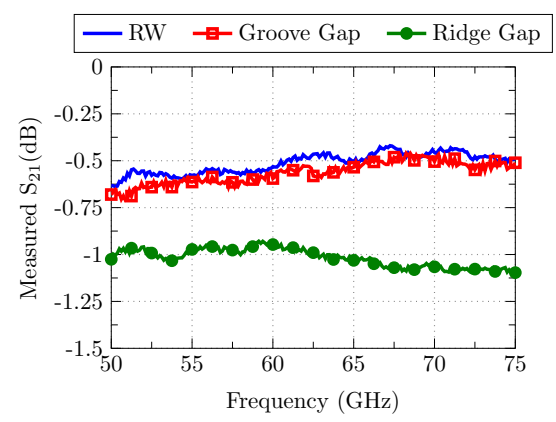

(b)

Figure 1.20: Measured reflection coefficients $S_{11}$ and transmission coefficients $S_{21}[2]$.

\section{Filters}

Gap Waveguide technology can be used to develop components for mm-wave applications such as filters [148]-[158], diplexers [159]-[161], hybrid couplers [162][164] or power dividers [134]. The advantages come from the low losses and its contactless nature. Within the different GW topologies, for the components mentioned above, the preferred one is GGW. The reason is that the GGW shows similar performance to the standard rectangular waveguide (RW). This feature suggested the GGW as an ideal alternative to the RW for mm-wave frequencies since ensuring correctly a perfect metal contact is very challenging. Strictly speaking, the behavior of GGW and RW are not identical, but they are similar enough to assume similar performance in most cases. This favors the computational time since the structures can be designed as RW to, later, change the metallic walls by the bed of nails and perform a fine-tuning procedure.

As can be deduced from the huge number of indexed publications, there is a great interest in the study of filters, and lately, also diplexers in GW technology since a lot of these components have been built so far at different frequency ranges. One of the first GGW filters can be found in [154]. In that work, a 61-GHz narrow-band filter implemented with GGW high-Q cavity resonators is described.

The fact that the authors chose high-Q resonators was precisely in order to exploit the properties of Gap Waveguide technology in full. As it is well-known, the losses that determine the unloaded $\mathrm{Q}$ of the resonator are composed of two main factors: metal loss and leakage loss. The metal losses are usually related to the volume of the resonator and conductivity of the material used to build 


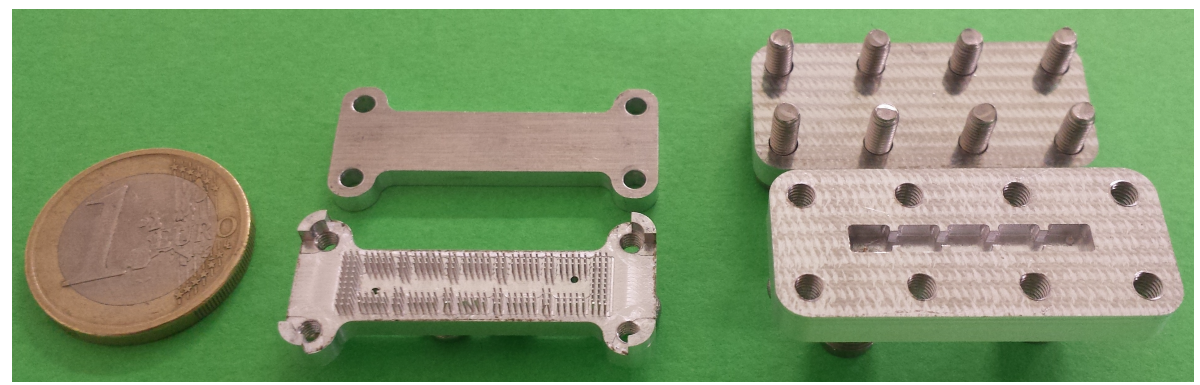

Figure 1.21: Manufactured filters: GGW (left-hand side), RW (right-hand side) in $[155]$.
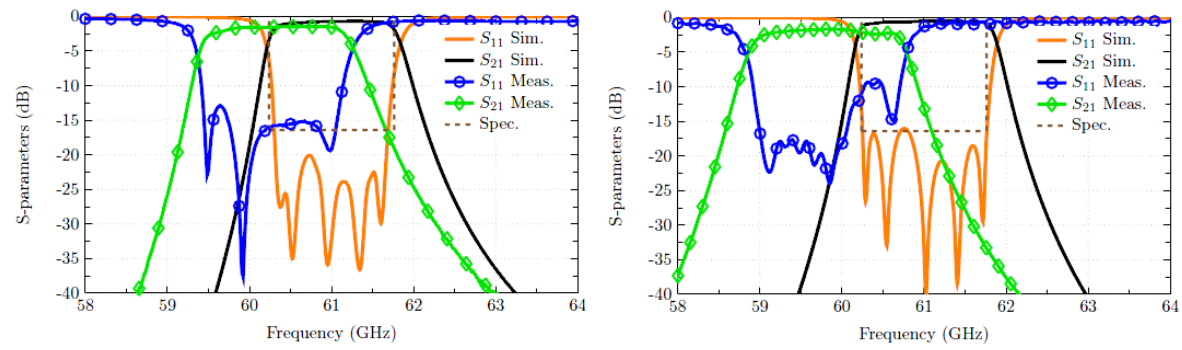

Figure 1.22: Comparison between the measured response and the optimum simulated response. (Left) GGW filter; (Right) Rectangular waveguide filter [155].

the resonator. The leakage losses are related to the leak of energy through the tiny gaps formed due to mechanical imperfections of the metal split block sections. Ideally, there are no losses due to leakage but in practice, leakage losses may become important.

Therefore, in that publication, a 5th-order Chebyshev filter with coupled resonators is designed and experimentally compared with an identical H-plane rectangular waveguide filter manufactured in [155]. That study revealed the robustness GGW offers over RW since the GGW filter response remains invariant after reassembling, whereas the rectangular waveguide filter worsened its response. These filters are shown in Fig. 1.21, and the measurements are compared in Fig. 1.22.

Since a mechanically flexible narrow-band diplexer is a very critical component in a full duplex communication link, there is a need for developing new types of high-Q filters which are more robust regarding mechanical assembly. With this in mind, the next step is to integrate the diplexer with other modules of passive components such as antennas as presented recently in [66]. High-Q 
filters open up the possibility of placing diplexer filters as integrated modules together with RF electronics and antennas.

\section{Transitions}

Transitions between gap waveguides and other standard transmission lines play an important role and are necessary modules for gap waveguide components. These transitions are also important from the measurement point of view, while evaluating the performance of the GW components. The motivation, implementation, and results of new transitions will be explained in depth in Chapter 2. In brief, three types of transitions are the most used today: Microstrip to ridge gap waveguide, coaxial to ridge or groove gap waveguide transition and rectangular to ridge gap waveguide. The most relevant example in the framework of this work is the latter one. This transition has been used several times, mainly to feed high-gain antennas ([72],[75] and [165]-[167]) where the feeding is placed at the back of the antenna. By tuning a few parameters, both types of waveguides can be matched. Good performance can be achieved. For example, in [72] results show about $17 \%$ reflection coefficient bandwidth for an $\mathrm{S}_{11}$ below $-20 \mathrm{~dB}$ in the frequency band of 56-66 GHz. The transition RW-RGW, depicted in Fig. 1.23, is far from being optimal since it requires machining a suspended stub, not easy to manufacture using, for example, milling techniques. However, it is true that the boom of additive manufacturing can alleviate this type of problems [168]. This transition was not characterized or measured separately, so it is hard to isolate its performance from the complete system in which it is employed.

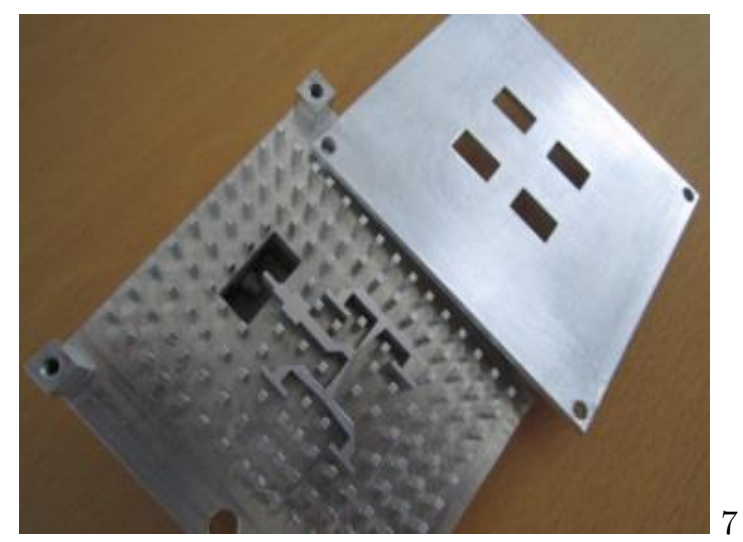

Figure 1.23: Transition RW-RGW used in a subarray $2 \times 2$ in [72]. 


\section{Packaging}

Most of the mm-wave and microwave systems have to operate at outdoor, under uncertain atmospheric conditions such as rain, wind, snow. Therefore those systems have to be sheltered suitably to protect against mechanical stress and environmental conditions. Likewise, to satisfy the smaller size and form factor requirements for mm-wave microwave modules, since a large number of electronic components must be placed into a reduced area. For such high-density microwave modules operating at mm-wave frequency range, $\mathrm{RF}$ packaging is more and more essential regarding isolation and interference suppression.

While previous topologies modify the periodic structure to enable propagation channels, now, the GW will be used for packaging purposes. Until now, the lid had always been a smooth, metallic plate. In this case, the top metal plate is replaced by nails facing down, towards the circuit to be packaged.

The first proposal of using a bed of nails for packaging purposes was presented in [169]. Later this novel idea was applied to a multiport antenna feed network in order to improve the performance of a coupled line microstrip filter [170]-[171]. In all cases, it was detected that unwanted cavity modes and surface waves were efficiently suppressed within the band of interest. Recently, several other periodic structures, other than traditional bed of nails, such as periodic metal springs or periodic zigzag lines printed on a substrate have been used to create the AMC condition for such kind of application [172]-[173].

\section{Antennas}

Antenna arrays are being the most prolific area of research within the Gap Waveguide technology. The most developed types have been those based on slots [174]-[182]. Nonetheless, other radiating elements such as horns [183], microstrip patches [184], leaky-wave antennas [185]-[186], Rotman lenses [187] or even Phased-Arrays [188] are found in the literature as well.

GW slot array antennas for mm-wave band have become a relevant topic thanks to the low loss and the contactless nature of the gap waveguides. Moreover, their planar profile results very suitable for many applications. The usual challenge with this kind of antennas is to design and manufacture corporate feeding networks which are compact enough to feed a $2 \mathrm{D}$ array properly. Although a series-fed slot-array is the simplest option and it is used sometimes, its most significant drawback its narrowband performance [189].

These initial works have been focused on small demonstrators, composed of $2 \times 2$ or $4 \times 4$ and later on high-gain antennas with, typically, $16 \times 16$ radiating elements, with aperture efficiencies of more than $70 \%$ and gain values above 32 dBi. Compared to similar antennas implemented in other technologies, such as SIW or LTCC (Low-Temperature Co-fired Ceramic), the gap waveguide slot arrays provide higher gain and efficiency. Gap Waveguide arrays are usually 


\section{CHAPTER 1. INTRODUCTION}

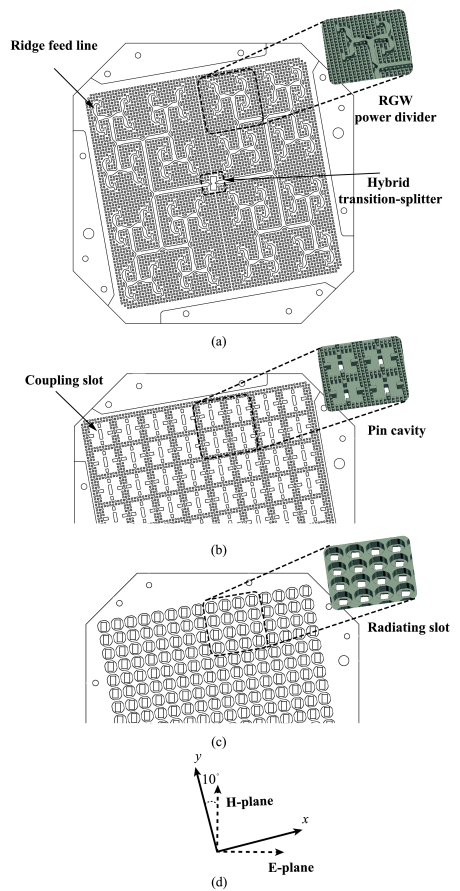

Figure 1.24: Configuration of the antenna proposed in [75].

based on the use of aperture antennas (either slots, horns or open cavities) which are fed by corporate networks made in one of the afore-discussed versions of gap waveguide technology, i.e., microstrip, inverted microstrip, ridge or groove. Commonly, these antennas stack several layers (bottom layers for the corporate-feed network dividing the power, and a top layer for the radiators and polarizers).

As slot arrays are the most developed array type and also the one most related to this work, the antenna presented in [75] is taken as a representative example. Fig. 1.24 shows a high-gain, high-efficiency, and broadband corporate-fed slot array antenna at V-band. That antenna consists of three metal layers (feed, coupling and radiating) without the need for electrical contact between them. The prototype consists on $16 \times 16$ cavity-backed slots. The antenna, manufactured by Electro-Discharge Machining (EDM) [190], is shown in Fig. 1.25. Antenna measurements show wide impedance bandwidth and good antenna efficiency. The antenna feeding network is made with RGW.

In [191], this same 3 -layer scheme is followed in a $8 \times 8$ patch antenna array with high gain and high radiation efficiency. The measured efficiency is remark- 


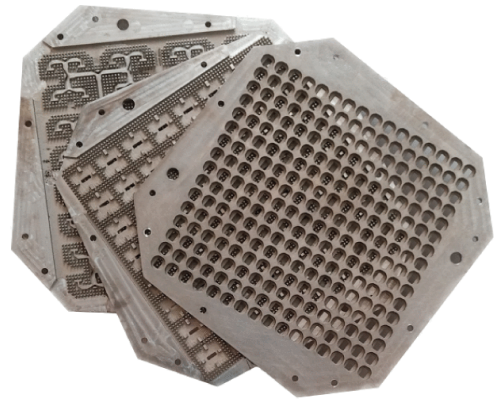

(a)

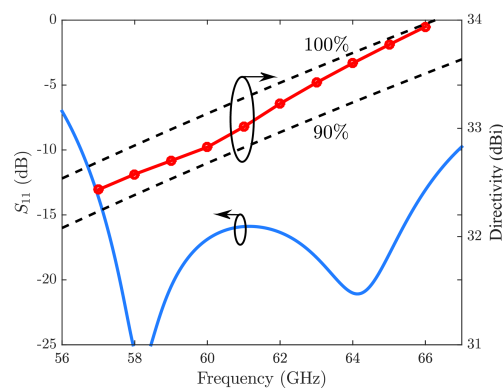

(b)

Figure 1.25: (a) Three-layer corporate-fed $16 \times 16$ slot array antenna and (b) Measured results [75].

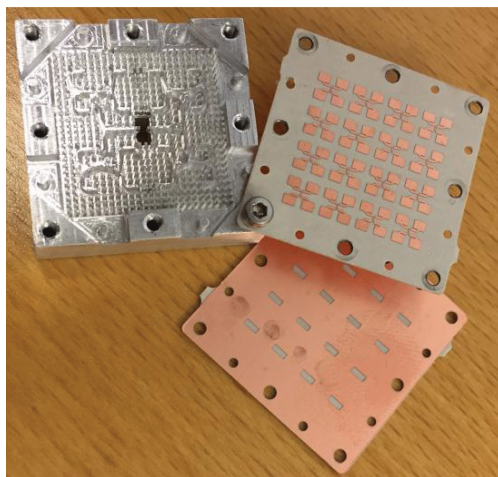

(a)

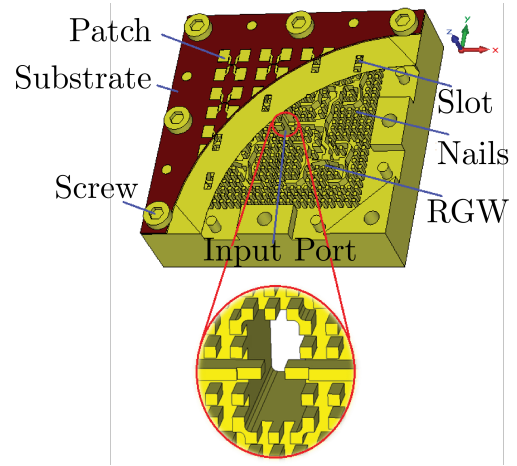

(b)

Figure 1.26: (a) A Gap Waveguide-Fed Wideband Patch Antenna Array for $60-\mathrm{GHz}$ in [191].

able, close to $80 \%$ despite using radiating printed patches. Fig. 1.26 shows the fabricated antenna and its measurements. Also examples of GW arrays fed with GGW or inverted microstrip GW have been developed (Fig. 1.27). A good summary of these contributions is described in [69].

The main drawback of these structures is precisely the need for the intermediate layer. In Chapter 4, antennas hosting the feed and the coupling layer in the same plane are presented. This feature will provide lighter, cheaper and lower profile antennas. 


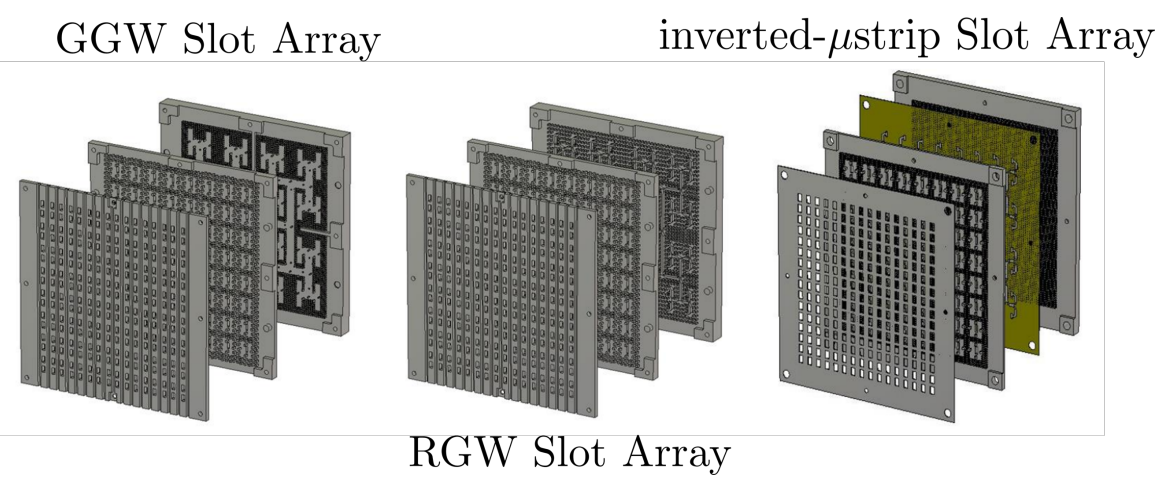

Figure 1.27: Schematics of three $16 \times 16$ slot array designed in gap waveguide technology. [69].

\section{$1.3 \quad$ Objectives}

The global objective of this Thesis is to develop antennas and corporate-feed networks in the mm-wave band for communication applications, with a particular emphasis on satellite applications, both fixed and mobile. This thesis is associated with a research project funded by the Subprogram of Research Projects of the Ministry of Economy and Competitiveness (Spain) within the framework of the State Plan for Scientific and Technological Research and Innovation 2013-2016.

One of the problems for achieving high-gain low-cost antennas in the mmwave band such as microstrip or stripline, is its limited efficiency. On the other hand, if waveguide-based solutions are chosen, the problem then is the weight, the machining precision and ensuring good electrical contact between the pieces.

Also, new antenna concepts are demanded to have ambitious performance concerning impedance bandwidth and polarization purity. Therefore, the primary challenge of this thesis is focused on developing systems with better integration between the distribution networks and the radiating elements as well as achieving it at a reasonable cost for civil applications.

In this context, the following specific objectives of this thesis were established:

1. Design of low-profile antennas with high gain and low losses for millimeter wave applications:

a. New concepts of antennas with higher impedance bandwidth, better directivity and cross polarization. 
b. Simpler low loss feeding networks reducing the number of layers.

c. Optimization of impedance, illumination and axial ratio of antennas in a larger bandwidth.

2. Research of new guiding architectures in the mm-wave band:

a. Development of new concepts of corporate-feed networks using Gap Waveguide technology.

b. Design of optimal transitions between different types of GW.

3. Validation of prototypes:

a. Measurement of prototypes, metrology studies and assessment of the experimental results obtained.

b. Exploration of new techniques such as additive manufacturing with the aim of lowering the weight and cost of the antennas as well as enabling new 3D topologies.

\subsection{Methodology}

The first phase of the thesis was devoted to the compilation of bibliographical revision. As background, the starting point is based on the experience acquired during the implementation of a previous project. This project, prior to this Thesis, was focused on the development of a multilayer antenna with circular polarization in the Ka-band for SATCOM on the move applications.

The second phase was dedicated to the implementation of radiating elements and corporate-feed networks based on Gap Waveguide in the mm-wave band. In this second phase, the full-wave electromagnetic simulators HFSS and CST has been used. Furthermore, a research stay was also carried out at the Chalmers University of Technology (Sweden) in the equator of the development of this Thesis. There, it was possible to deepen the knowledge of GW technology as well as to know another environment and way of working that enriches the present work.

Finally, the third phase has been focused on the measurement and manufacture of prototypes in Gap Waveguide technology. Fabrication of the antennas has been realized mainly at the UPV facilities, though, some instances have been manufactured externally. On the other hand, the experimental validation has been carried out at the UPV anechoic chamber. Some prototypes were also measured by Universidad Politécnica de Madrid (UPM) and the astronomical observatory of Yebes. In addition, this Thesis has allowed to keep an 
active collaboration with the Universidad Carlos III of Madrid (UC3M) and the Universidad Pública de Navarra (UPNA).

\subsection{Structure of the Thesis}

This thesis is organized as follows:

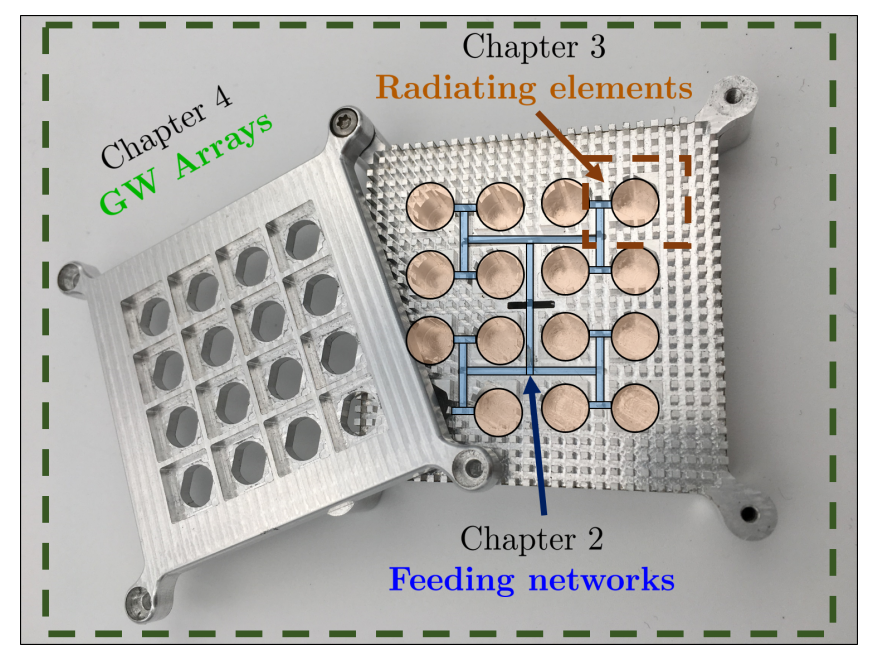

Figure 1.28: Structure of the Thesis illustrated in an array.

Chapter 2 focuses on explaining the distribution networks in Gap Waveguide that have been used as feeders for the antennas proposed in this Thesis. Firstly, a review of existing high impedance surfaces is made. Then, three GW networks are detailed.

Chapter 3 describes two different radiating elements in Gap Waveguide. The first one consists of a coaxial cavity that is formed by lowering one of the nails that make up the fakir bed, typical of GW technology. The second of the proposed elements is a cylindrical cavity that efficiently radiates the power and also presents versatility to stack different layers, achieving antennas with dual and circular polarization.

Chapter 4 presents the most outstanding prototypes developed during the realization of this work. Four of these models have been manufactured and their features validated. These Gap Waveguide Arrays are working in the mmwave band, mainly in the Ka-band, although there is also a $\mathrm{V}$ band model. 
Their architecture is based on the networks explained in Chapter 2, together with the radiating elements explained in Chapter 3.

Chapter 5, exposes the conclusions and the future research lines of the work done and points out the prospects of the technology developed. 


\section{Chapter 2}

\section{Corporate feeding networks based on Gap Waveguide}

"Ever tried. Ever failed. No matter. Try again. Fail again. Fail better."

— Samuel Beckett

This second chapter presents the corporate feeding networks based on Gap Waveguide used or developed in this thesis. Before delving into these networks, the chapter will focus on the artificial magnetic conductor (AMC) environment surrounding them. The AMC is a type of high-impedance surface (HIS). For a given frequency band, the surface impedance becomes very high and the transverse magnetic field component vanishes, that is, the structure behaves as a magnetic conductor [192]. In Gap Waveguide technology, the bed of nails is employed to realize such high impedance surface. But there exists other practical AMC realizations deserving of consideration in the context of GW technology. In this chapter, the performance of the bed of nails will be studied taking into account practical design considerations and its relationship with other HIS types. Finally, the different types of GW networks used in this thesis will be presented.

\subsection{Bed of nails}

The fundamental parameters involved to achieve a parallel-plate stopband for wave propagation are shown in depth. When a periodic structure behaving as an AMC is located close enough to a metal plate (with an air gap smaller 

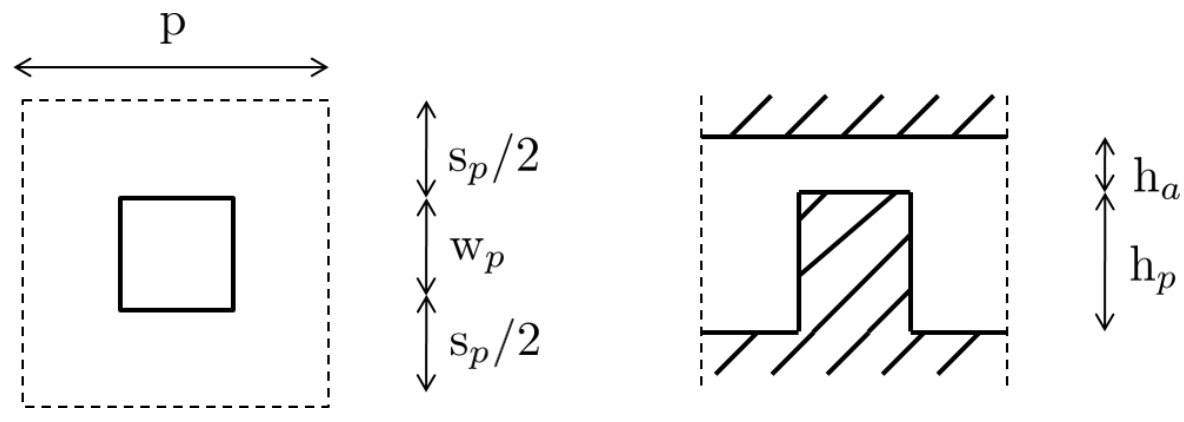

Figure 2.1: Basic cell used to study the stopband performance of the Gap Waveguide. Top (left) and cross-section (right) views are showed.

than $\lambda / 4$ ), no wave can propagate between those surfaces within a certain frequency band (stopband). The lower and upper cutoff frequencies will define the bandwidth of such stopband, which will in turn depend on the geometric parameters of the periodic structure. Regarding the bed of nails, the dimensions governing the stopband of the parallel-plate propagation are the nail height $\left(h_{p}\right)$, nail period $(p)$, nail width $\left(w_{p}\right)$ and the distance from the top of the textured surface (AMC) to the upper plate $\left(h_{a}\right)$.

The choice of the most appropriate configuration for the bed of nails is not a trivial issue. Depending on the particular design, different trade-offs between the involved geometric parameters will be taken into consideration. In this thesis, mainly focused on large arrays fed by waveguide networks, the bed of nails design plays a critical role to attain a satisfactory performance. Fig. 2.1 shows the geometric parameters involved. A preliminary analysis of the impact of these parameters on the stopband bandwidth is reported next. This study will serve to understand the design decisions taken throughout this thesis. Despite a center frequency of $30 \mathrm{GHz}$ has been set as the reference, the final conclusions are drawn in terms of the free-space wavelength $\lambda_{0}$ for the sake of generality.

\section{Nail height $\left(h_{p}\right)$}

Firstly, a parametric study with different $h_{p}$ values was done to study its effect on the stopband. All other relevant parameters kept fixed: periodicity $(p)$ was set to $0.2 \lambda_{0}, w_{p}$ to $0.1 \lambda_{0}$ and $h_{a}$ to $0.2 \lambda_{0}$. The dispersion diagram of the parallel-plate structure, showing the different propagating modes, is represented in Fig. 2.2.

As already mentioned in Chapter 1 , the height of the nails must be approximately $\lambda_{0} / 4$ to transform a short circuit (PEC) to an open circuit (PMC). From 


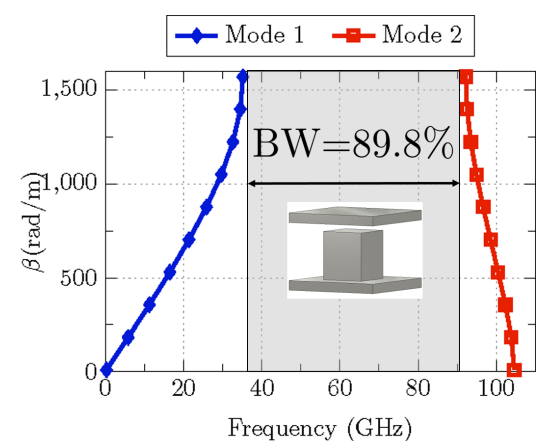

(a)

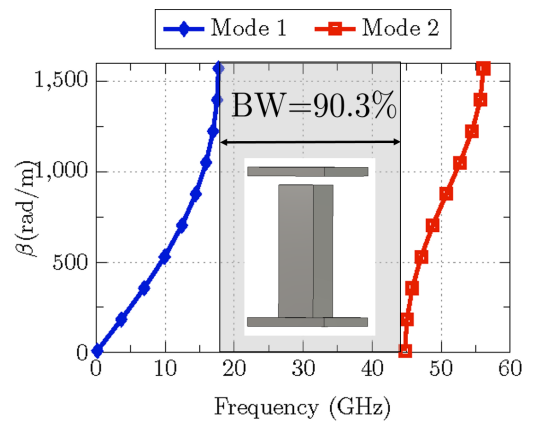

(c)

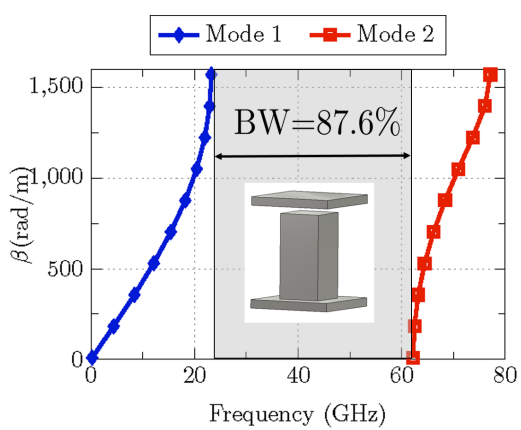

(b)

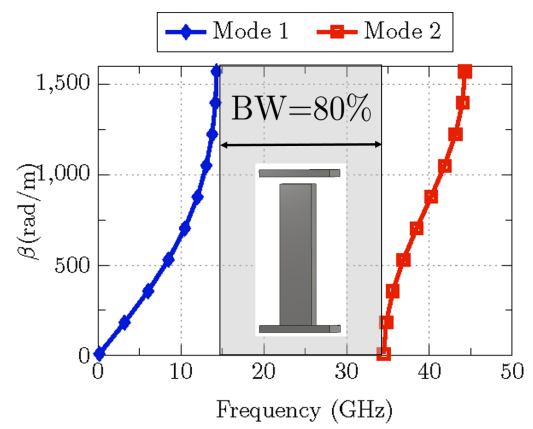

(d)

Figure 2.2: Study of the stopband for several nail heights: (a) $h_{p}=0.1 \lambda_{0}$, (b) $h_{p}=0.2 \lambda_{0}$, (c) $h_{p}=0.3 \lambda_{0}$, (d) $h_{p}=0.4 \lambda_{0}$.

this fundamental principle, the most logical nail height would be $2.5 \mathrm{~mm}$, since it is a quarter wavelength at $30 \mathrm{GHz}$. However, it is interesting to see how the stopband varies if this height is not exactly that value. Sometimes, for design or manufacture reasons it is more convenient to choose lower or higher nail heights. A height of $1 \mathrm{~mm}$ (Fig. 2.2a) is totally invalid for a $30 \mathrm{GHz}$ design since the stopband starts at almost $40 \mathrm{GHz}$, being more appropriate for $\mathrm{V}$ band $(60 \mathrm{GHz}$ ). As expected, a height of $3 \mathrm{~mm}$ (Fig. 2.2c) may seem the best option since the band is centered at $30 \mathrm{GHz}$ and there is enough margin on both sides. However, the higher the nails, the more time of machining is needed in the fabrication process and more fragile they are. A height of $4 \mathrm{~mm}$ (Fig. 2.2d), though it is strictly not outside the band, would not be advisable since a higherorder mode starts to propagate at $35 \mathrm{GHz}$. Moreover, an $h_{p}=4 \mathrm{~mm}$ would not favor an efficient manufacturing. 


\section{CHAPTER 2. CORPORATE FEEDING NETWORKS BASED ON GAP WAVEGUIDE}

On the other hand, it is important to confirm that the relative bandwidth is hardly affected by the different nail heights and just a slight frequency shift is manifested. As a general rule, it can be concluded that the parameter $h_{p}$ should be between $0.2 \lambda_{0}$ and $0.3 \lambda_{0}$.

\section{Nail width $\left(w_{p}\right)$}

Nails are commonly square in shape, though some works opt for cylindrical nails [193]. Typically, the height-to-width ratio of the nail is 2:1. Different widths are analyzed here to assess its impact on the stopband, showing the results in Fig. 2.3.

From the results it is deduced that the most optimal solution is the one presented in Fig 2.3d, not only because of the larger relative bandwidth but also because it is efficient in terms of manufacturing. In Fig. 2.3f, for the thickest and stiffest nail, a mode is close to the lower cutoff frequency. In that case, even small deviations in the manufacturing process might alter the expected device performance. Thus, as valid options for a $30 \mathrm{GHz}$ design, values of $w_{p}$ from $0.75 \mathrm{~mm}$ to $1.25 \mathrm{~mm}$ are possible when keeping fixed $h_{p}=2 \mathrm{~mm}$. Extrapolated to any frequency, nail height-to-width ratio should be between 1.6 and 2.6.

\section{Periodicity of nails $(p)$}

A third design parameter is the periodicity of nails, whose stopband results are shown in Fig. 2.4. The case of Fig. 2.4a would not be a good option. First of all, it would entail a very dense nail bed, which once again, it is not desirable in terms of manufacture. More importantly, the lower cutoff frequency is very close to $30 \mathrm{GHz}$. However, sometimes it is convenient to have a denser bed of nails. For compact corporate networks, branches are close to each other and at least two rows of nails are necessary to ensure good isolation. For this reason, most corporate networks adopt an option similar to that shown in Fig. 2.4b. Nonetheless, for less intricate networks, larger periods are possible, leading to simpler beds with fewer nails.

\section{Air gap $\left(h_{a}\right)$}

Usually, the air gap is around $\lambda / 20$ but there is not a well-established criterion except that it must be narrower than $\lambda / 4$. Therefore, this choice is usually left to the designer's discretion. In recent years it is not uncommon to see many designs without an air gap, also called as zero-gap condition. One might think that it contradicts the basic principles of GWs, but in fact, it is just the opposite. The zero-gap condition exhibits all the advantageous properties of GW technology. In addition, if a zero-gap structure has non-contact zones (for example due to inaccuracies in machining), its performance is hardly affected. 


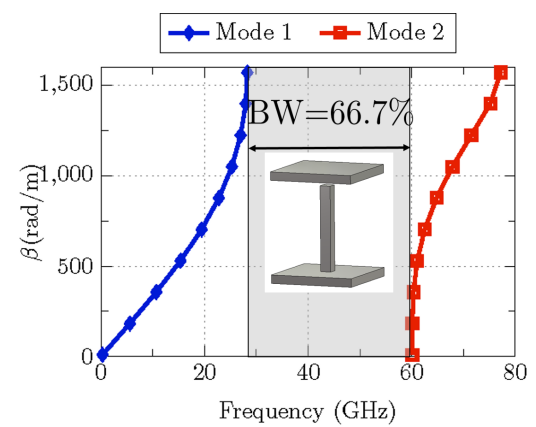

(a)

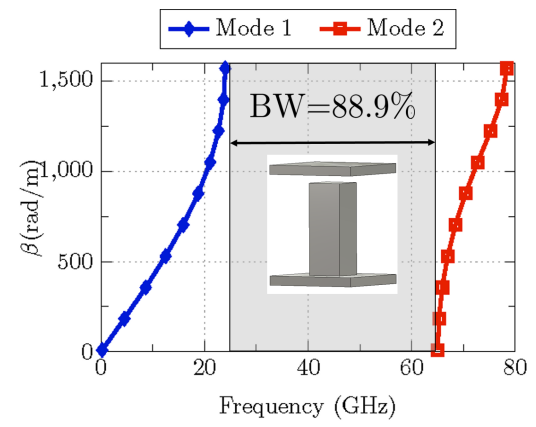

(c)

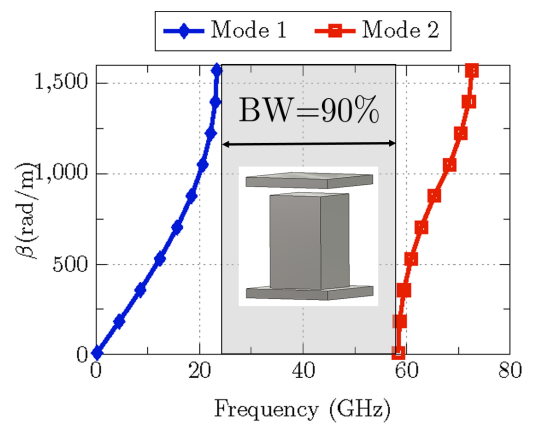

(e)

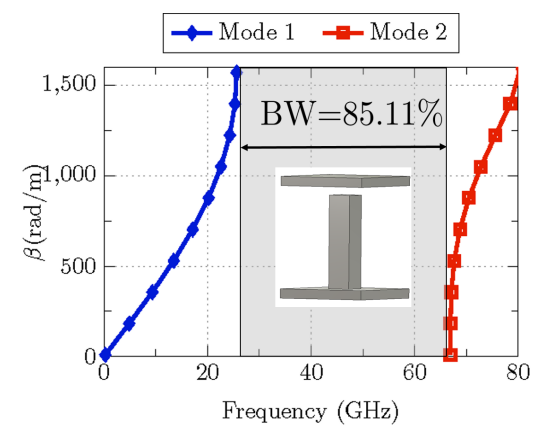

(b)

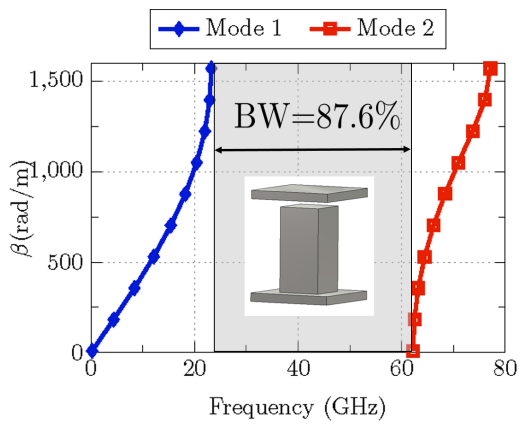

(d)

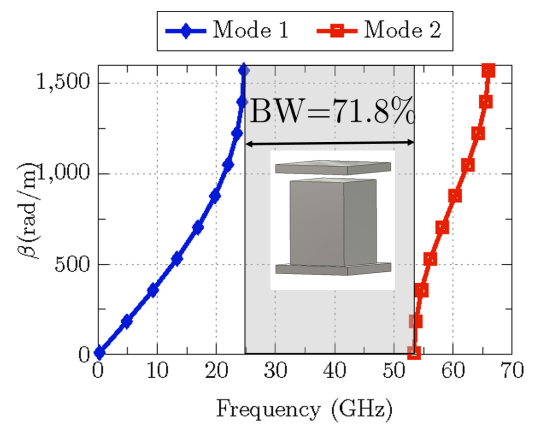

(f)

Figure 2.3: Study of the stopband for several nail widths: (a) $w_{p}=0.25 \lambda_{0}$, (b) $w_{p}=0.05 \lambda_{0}$, (c) $w_{p}=0.075 \lambda_{0}$, (d) $w_{p}=0.1 \lambda_{0}$, (e) $w_{p}=0.125 \lambda_{0}$, (f) $w_{p}=0.15 \lambda_{0}$. The rest of the parameters are $p=0.2 \lambda_{0}, h_{p}=0.2 \lambda_{0}$ and $h_{a}=0.2 \lambda_{0}$. 


\section{CHAPTER 2. CORPORATE FEEDING NETWORKS BASED ON GAP WAVEGUIDE}

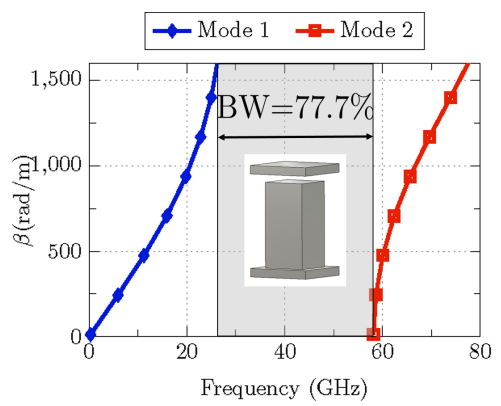

(a)

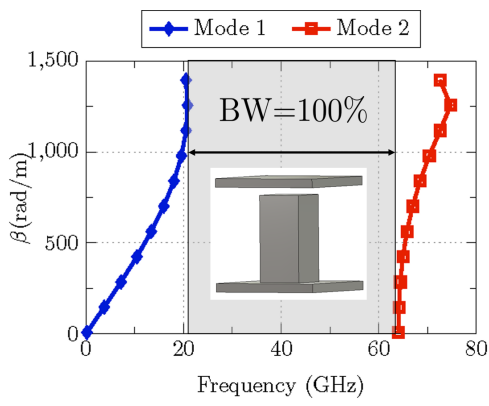

(c)

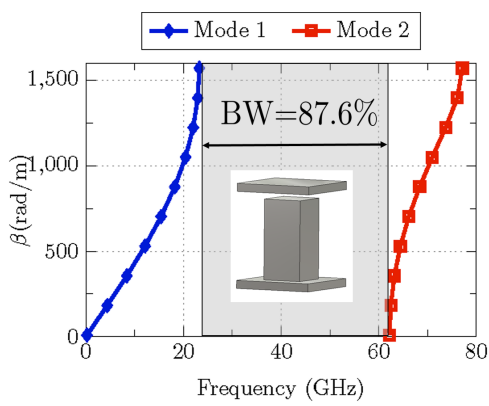

(b)

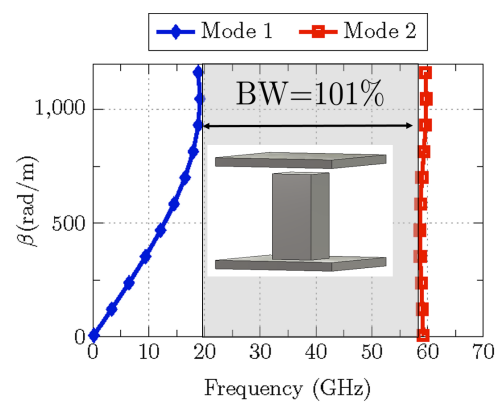

(d)

Figure 2.4: Study of the stopband for different nail periodicity: (a) $p=1.5 \mathrm{~mm}$, (b) $p=2 \mathrm{~mm}$, (c) $p=2.5 \mathrm{~mm}$, (d) $p=3 \mathrm{~mm}$. The rest of the parameters are $w_{p}=1 \mathrm{~mm}, h_{p}=2 \mathrm{~mm}$ and $h_{a}=0.2 \mathrm{~mm}$.

This will be demonstrated below. The wider the air gap, and the closer it gets to the quarter-wavelength, the smaller the stopband bandwidth will be, as shown in Fig. 2.5.

\section{Parametric study as a function of periodicity}

Results seen so far performed a single parameter sweep, setting the rest fixed, being able to verify how these values $\left(w_{p}, h_{p}, p, h_{a}\right)$ affect the stopband. In the following figures a more generic study is presented. Now the behavior of the relative bandwidth is studied varying $h_{p}, w_{p}$, and $h_{a}$, but for several values of parameter $p$.

As seen in Fig. 2.6a the narrower the air gap, the greater the stopband bandwidth. As for the nail width, something remarkable happens. If periodicity is small, that is, the bed of nails is dense, periodicity becomes the 


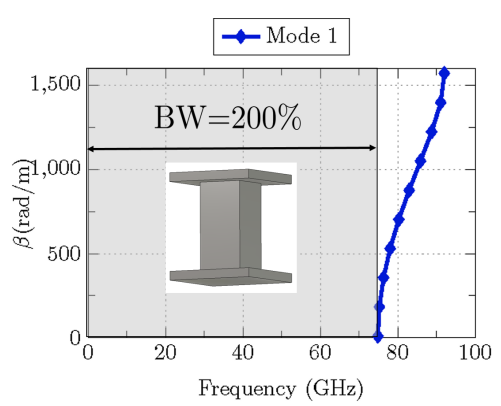

(a)

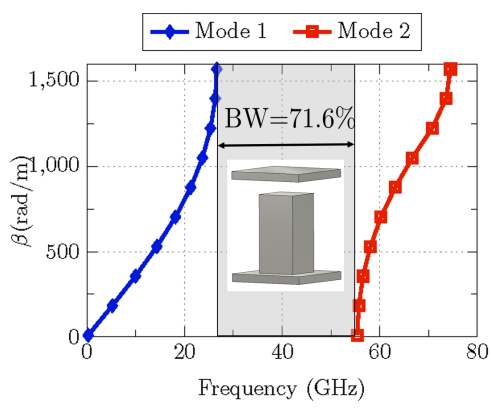

(c)

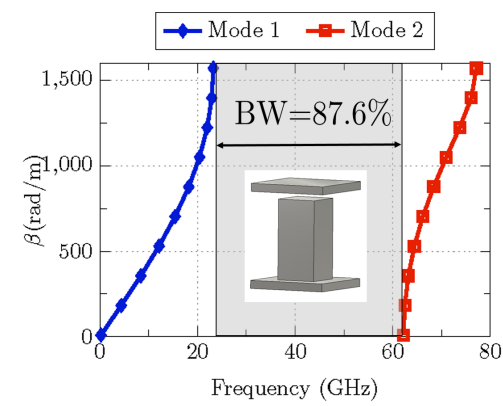

(b)

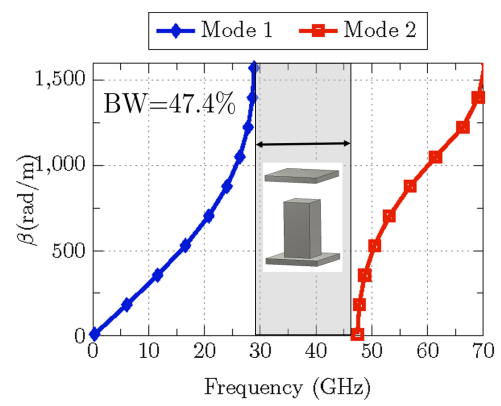

(d)

Figure 2.5: Study of the stopband for different gap heights: (a) $h_{a}=0 \mathrm{~mm}$, (b) $h_{a}=0.02 \lambda_{0}$, (c) $h_{a}=0.04 \lambda_{0}$, (d) $h_{a}=0.08 \lambda_{0}$. The rest of the parameters are $w_{p}=0.1 \lambda_{0}, h_{p}=0.2 \lambda_{0}$ and $p=0.2 \lambda_{0}$.

determining factor and the nail height hardly affects the stopband. When the bed is less dense, height does become a parameter to be taken into account. Finally, perhaps one of the factors of less importance is $w_{p}$. From these results it is understood why using different shapes (cylindrical, square) is not a relevant factor for the stopband performance. This parametric study will allow to properly choose the bed of nails parameters when designing optimal distribution networks.

\section{Rule of thumb}

As rule of thumb, nail height should be between $0.2 \lambda_{0}$ and $0.3 \lambda_{0}$. Periodicity of nails can oscillate between a very dense bed of nails $\left(p=0.1 \lambda_{0}\right)$ or a lowdensity one $\left(p=0.4 \lambda_{0}\right)$. Nail periodicity-to-width ratio should be between 2 and 3 . The air gap should be less than $0.25 \lambda_{0}$. Zero-gap is also acceptable since 


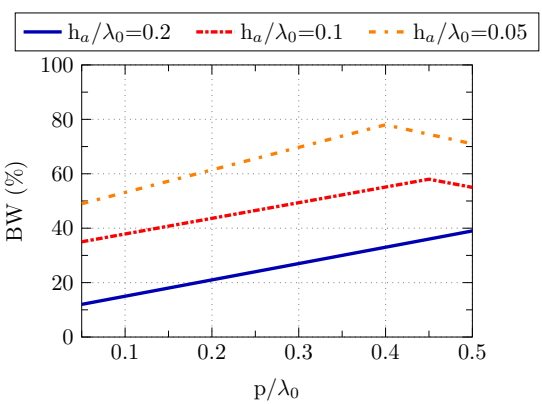

(a)

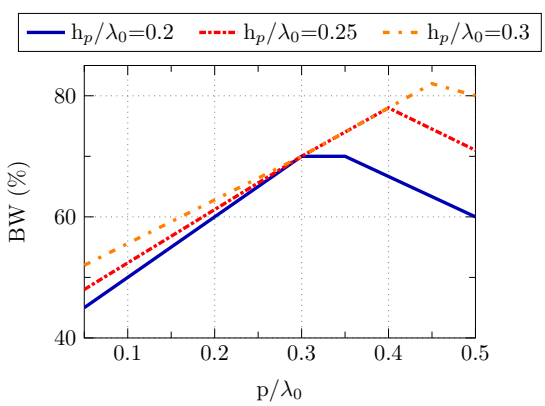

(b)

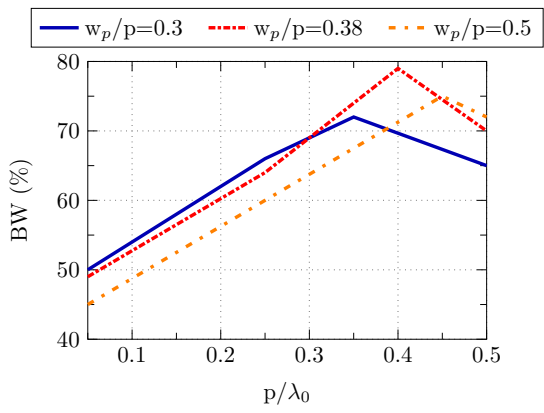

(c)

Figure 2.6: Parametric study as a function of periodicity.

the properties of the GW remain intact and has been taken into consideration in future designs.

\subsection{Other AMC Realizations}

It would be excessively lengthy to list all the different types of high impedance surfaces existing in the literature but at least let us take a small representative part. Beyond its infinite versions and modifications, in this section some of the most relevant realizations are presented, either because of the technology background (mushroom-type) or its direct connection with gap waveguide technology (bed of springs or glide symmetry). 

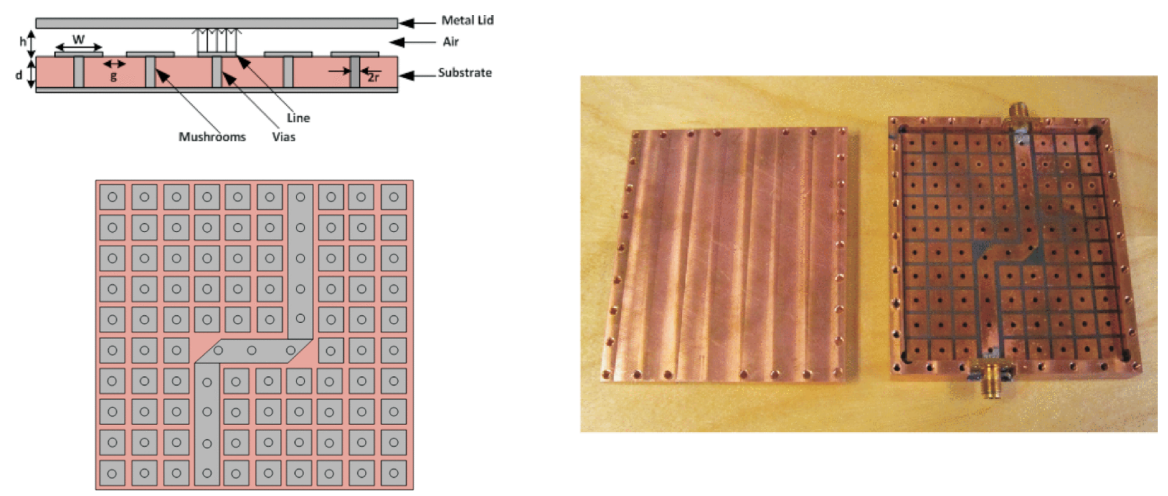

Figure 2.7: Front and top view a microstrip gap waveguide surrounded by a mushroom-type EBG surface and a manufactured prototype [194].

\section{Mushroom-type}

Mushroom-type EBG is a conventional three-dimensional EBG consisting of a solid patch with a cylindrical via to the ground. A side view of this structure is shown in 2.7. The transmission response of a mushroom-type EBG depends upon the size of the patch, via diameter, gap between the unit elements and substrate thickness and permittivity. Fig. 2.7 shows a manufactured microstrip gap waveguide surrounded by a mushroom-type EBG surface, presented in [194].

In [195], different patch shapes such as crosses, hexagons or even fractal forms are proposed with the aim of improving the bandwidth for different applications. An interesting study presented in [196], demonstrated that increasing the thickness of the vias does not improve the bandgap width but moves it to higher frequencies.

Another structure worth mentioning is the one made in [197]. It is an interleaved EBG structure with a smaller area than the conventional mushroom-like EBG structure. That new realization manifests a substantial improvement on bandwidth of $51.1 \%$ and miniaturization of $61.2 \%$ compared to the conventional approach. Another investigation [198], exploring similar possibilities, showed that by changing the position of the metallic vias from the patch center to its edge, the bandgap moves toward lower frequencies.

\section{Uniplanar EBG configurations}

Unlike the mushroom-type, the uniplanar EBG configurations have no vertical vias and are appropriate for thin substrate materials. Besides, this structure displays a reduced dependence on polarization and incident angle [199]. Re- 


\section{CHAPTER 2. CORPORATE FEEDING NETWORKS BASED ON GAP WAVEGUIDE}

sembling mushroom EBGs, uniplanar EBGs are also frequency-sensitive since it also responds to an equivalent LC network within a particular frequency band. However, in contrast to mushroom EBGs it is difficult to establish accurate LC equivalent models for uniplanar arrangements. Within the stopband, the texture performs as a high-impedance surface and stops the promulgation of surface waves [200]-[203]. In uniplanar EBGs, equivalent inductor L is synthesized as thin connecting lines in the EBG cell while capacitance $\mathrm{C}$ is due to gaps between adjacent cells.

\section{Bed of springs}

Some other periodic structures have been studied also for low-frequency applications where compact unit cells are needed, particularly in packaging applications. That is the framework where the bed of springs shown in Fig. 2.8 has been proposed [172], being an alternative to the bed of nails for low frequencies. The period of the helical structure can be sub-wavelength, and most importantly, due to the wire shape, the total height of the unit cell will also be small. The main parameters of this bed of springs are the height and diameter of the springs, height of the air gap, periodicity, and number of turns in each spring, as shown in Fig. 2.8a.

As commented in [172], the effect of the air gap height in this solution is not so critical as compared to the case of the bed of nails or mushrooms. It was demonstrated that, when springs are used, the upper limit of the stopband is almost unaffected by the air gap, while the lower limit increases a bit when increasing the gap. In any case, this parameter is not moving the stopband in a relevant way. This feature provides a valuable design flexibility when compared to the nails or mushroom-type EBG geometries. Such a good performance is due to the lower capacitive effect between the top of the springs and the upper plate, which makes the gap a factor of less importance. In short, this structure can be useful for packaging application. However, its manufacturing complexity is evident and it does not seem an adequate solution for the applications developed here.

\section{Zigzag wires}

In [173], a planar zigzag-wire structure is proposed, representing somehow the printed version of the bed of springs. This periodic zigzag structure is printed on several slices of thin dielectric boards, which are located vertically side by side to form the periodic surface shown in Fig. 2.9. In this structure the lower cutoff frequency appears when the electrical length of the wires is approximately $0.25 \lambda$ in free space, and the upper one when the spacing $h+g$ between the two metal plates is approximately $0.5 \lambda$. The stopband condition also pre- 


\subsection{Other AMC Realizations}
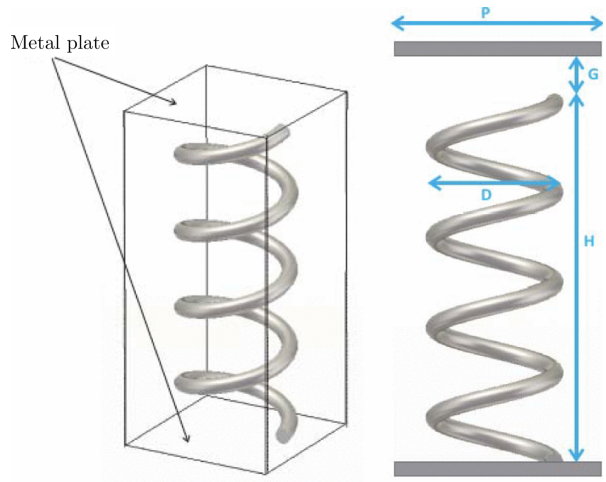

(a)
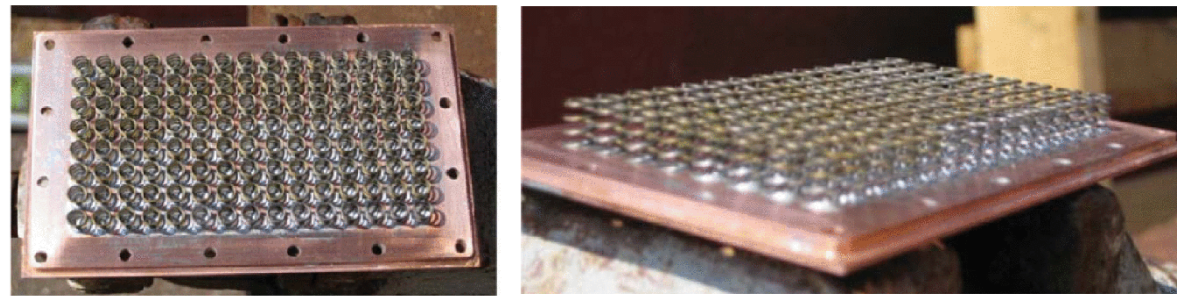

(b)

Figure 2.8: (a) Description of the bed of springs unit cell and all its parameters. (b) Manufactured bed of springs [172].
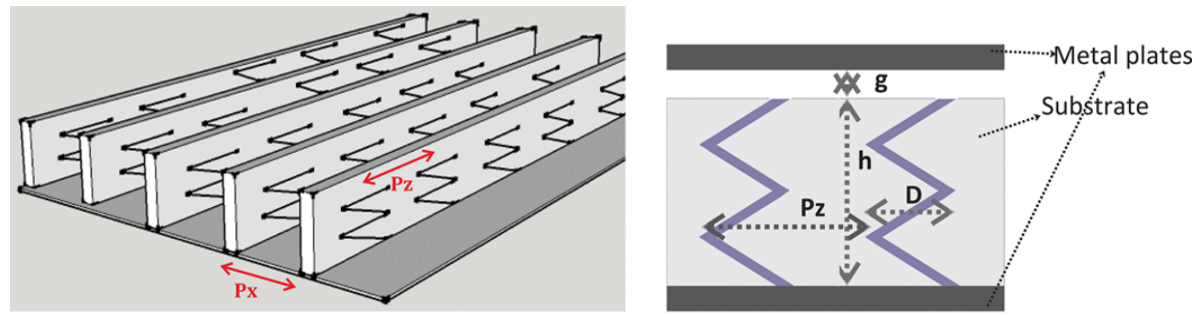

Figure 2.9: Zig-zag structure proposed in [173].

vents from radiation leakage from the small gap between the metal lid and the box walls, exhibiting a behavior similar to GW but employing a printed circuit. 


\section{CHAPTER 2. CORPORATE FEEDING NETWORKS BASED ON GAP WAVEGUIDE}

\section{Glide simmetry}

Glide symmetry is a novel cost-effective method to manufacture integrated waveguide structures at high frequencies. This concept makes use of a truncated glide-symmetric holey EBG structure [204]. The proposed EBG structure is only made of holes, which makes the manufacturing process easier with respect to the nails in gap waveguide technology. Among the advantages highlighted in [205], the higher accuracy and lower cost of manufacturing at high frequencies stand out. This technology represents a good alternative to GW as described in [206], though glide-symmetric structures are in general more bulkier.

Let us follow the evolution of the unit cells represented in Fig. 2.10. Fig. 2.10a shows a common bed-of-nails unit cell, and Fig. 2.10d its complementary version: a periodic repetition of squared holes in a metallic slab. In order to compare the holey structure to the bed of nails, the holes have been shaped as squares. However, the manufacturing process could be easier with cylindrical or conical shapes. Both cases have a top metallic plate separated by an air gap. Then, the new configuration is faced, as shown in Fig. 2.10b and 2.10e. Additionally, and most importantly, both metasurfaces must be shifted from each other by half a unit cell, as depicted in Figs. 2.10c and 2.10f.

Next, the dispersion diagram of both structures were compared to analyze their behavior. The dispersion diagram for the bed of nails configuration is represented in Fig. 2.11. Particularly, the dispersion properties for the first two modes are shown for the unit cells of Fig. 2.10a and 2.10c when the parameters are $\mathrm{l}=4 \mathrm{~mm}, \mathrm{~d}=0.5 \mathrm{~mm}, \mathrm{~h}=1 \mathrm{~mm}, \mathrm{~g}=0.1 \mathrm{~mm}$. Two incident angles are presented in each graph: $0^{\circ}$ and $45^{\circ}$. The dispersion diagram for the holey configurations of Fig. 2.10d and 2.10f are given in Fig. 2.11, again for those same two angular directions and for the following parameters: $b=0.5 \mathrm{~mm}, \mathrm{l}=4$ $\mathrm{mm}, \mathrm{h}=1.5 \mathrm{~mm}$, and $\mathrm{g}=0.1 \mathrm{~mm}$. The results are very similar to those obtained for the bed of nails. Thus, this novel structure, as the GW, alleviates the problem of electrical contact between two metal parts since the field will remain confined.

Typically, cylindrical holes are more used than the squared ones because they facilitate the manufacturing [207]. For example, it is really easy to drill holes in a metal plate. However, in order to achieve wider stopbands, holes must be made both on the bottom plate and on the top plate as discussed in [205]. Fig. 2.12 is revealing and demonstrates the need for two layers with holes facing one another. Note how this mandatory requirement of two plates to increase that initial narrow stopband leads to bulkier structures.

In order to demonstrate the shielding effectiveness of the proposed structure, an aluminum waveguide with a double $90^{\circ}$ bent (Fig. 2.13) was designed and measured in [205]. First, a conventional WR-19 rectangular waveguide was con- 


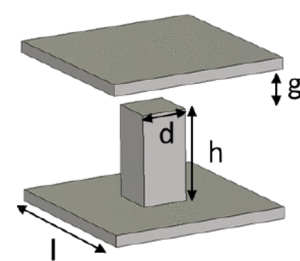

(a)

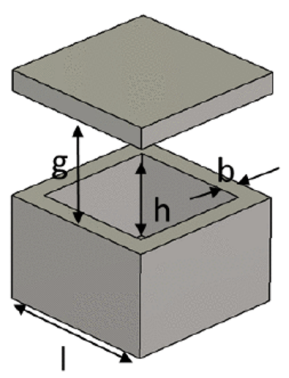

(d)

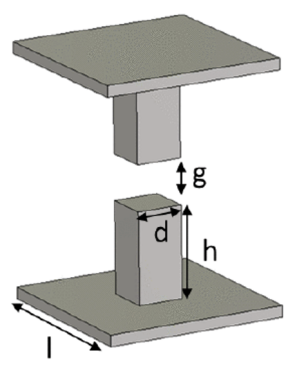

(b)

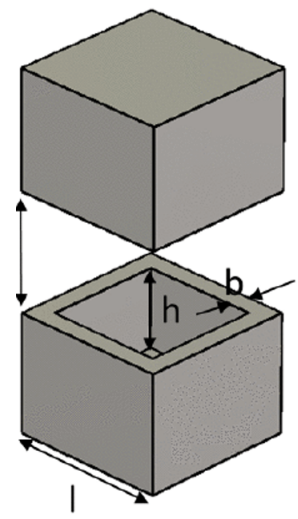

(e)

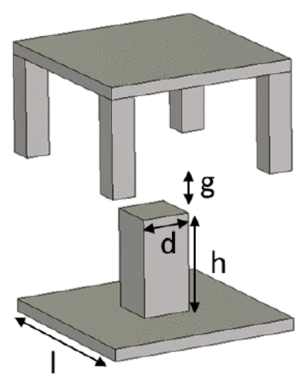

(c)

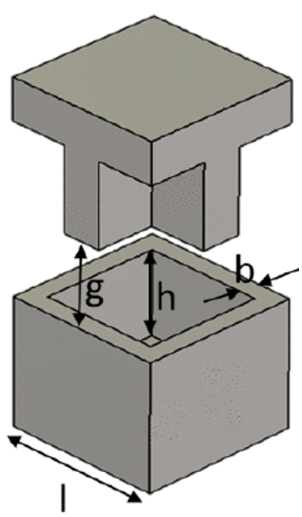

(f)

Figure 2.10: Metasurface configurations presented in [206]: (a), (d) single metasurface with a metallic top cover; (b), (e) doubled metasurface, face-to-face; (c), (f) doubled metasurface, half unit cell shifted; (a), (b), (c) bed of nails; (d), (e), (f) squared holes.

sidered. Later, a small gap of air was left and finally the glide-symmetric holey EBG was introduced. As presented in Fig. 2.14, even a very small gap excites parallel plate modes and produces a significant field leakage (Fig. 2.14b). However, by placing two rows of glide-symmetric holey EBG at both sides, almost perfect transmission in the operation frequency range of the EBG structure is achieved (Fig. 2.14c).

In conclusion, the glide-symmetric alternative is similar to the groove gap waveguide technology. For all the different methods of manufacturing, such as electro discharge machining (EDM), molding and casting, and CNC milling, 

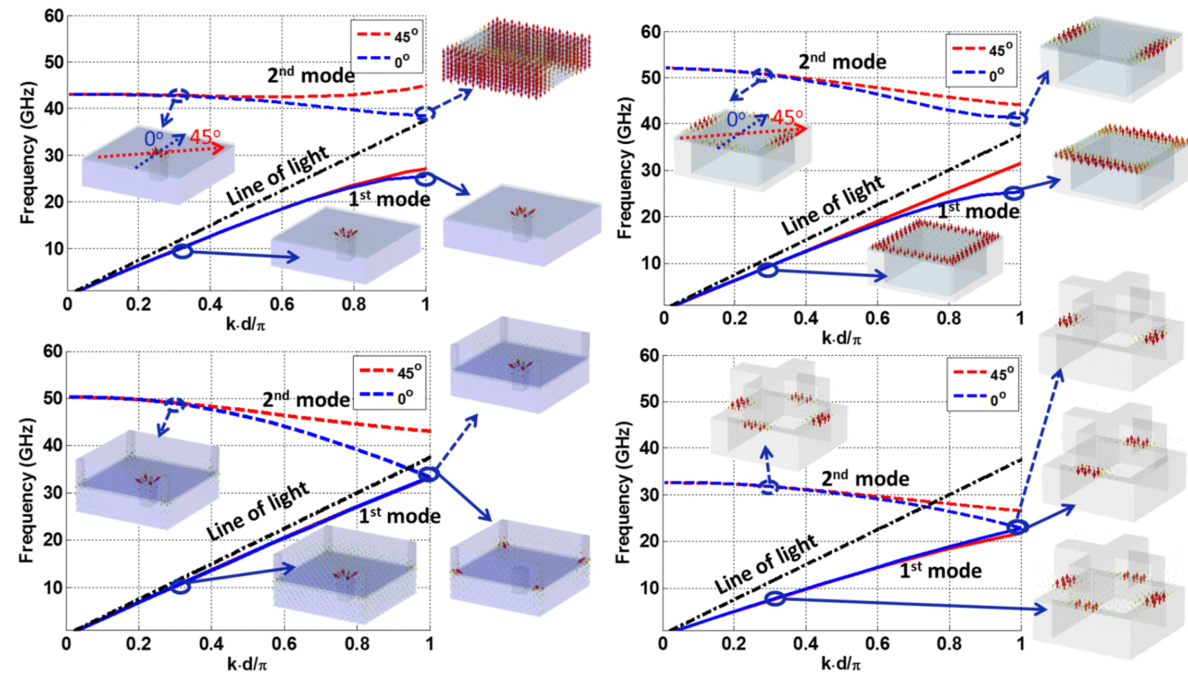

Figure 2.11: Dispersion of a metasurface under periodic conditions [206]: (left) Gap Waveguide, (right) Glide Symmetry.

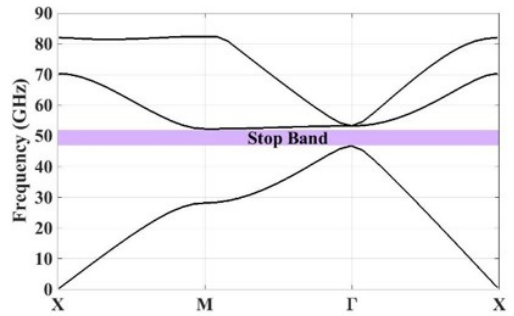

(a)

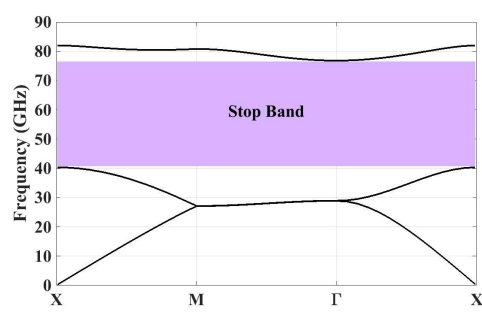

(b)

Figure 2.12: Dispersion diagram for two unit cells [205]. (a) Holes only on one plate. (b) Glide-symmetric holes on both plates.

the glide-symmetric holey EBG is potentially cheaper and easier than GGW at the same frequency band. 


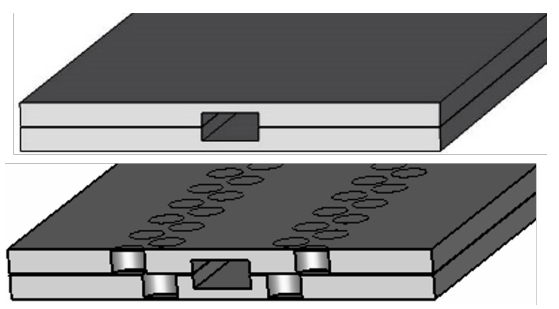

Figure 2.13: Rectangular waveguide: (Up) Conventional WR-19. (Down) Two rows of glide-symmetric holes surrounding the waveguide [205].

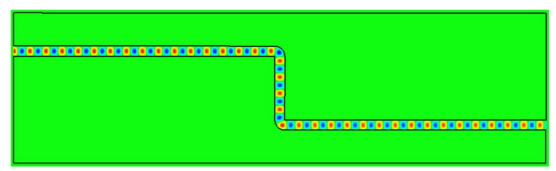

(a)

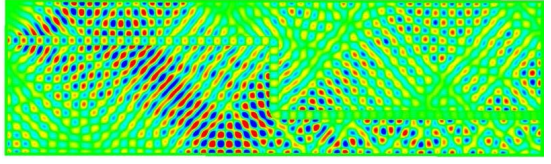

(b)

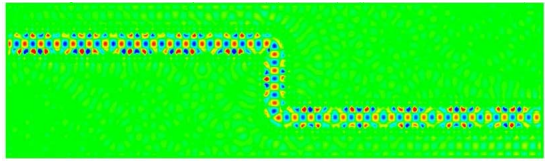

(c)

Figure 2.14: Normalized electric field distribution of (a) aluminum WR-19, (b) waveguide with an air gap on the side walls, and (c) waveguide with a glide-symmetric holey EBG [205].

\subsection{Contactless feature of GW}

The contactless feature of GW must be thoroughly reviewed since its a key aspect of the technology. To understand its potential, we take as an example two waveguides whose performance is assumedly equivalent: RW and GGW.

This behavior was firstly demonstrated in [209]. It was observed that the field distribution of the GGW fundamental mode is very close to that of a RW. In fact, GGWs have been successfully utilized in the past for waveguide slot arrays [210]. Also in [208], a study in that sense was carried out. In Fig. 2.15, propagation constant for the fundamental mode of a GGW was compared to that of a RW having the same cross section. Although curves manifest a slightly different slope, they can be made practically equal for a given frequency just by adjusting nails width and periodicity. The reflection coefficient was also compared (Fig. 2.16) and very good agreement is shown. The study done in [211] reached the same conclusion. It was shown that the GGW field distribution 


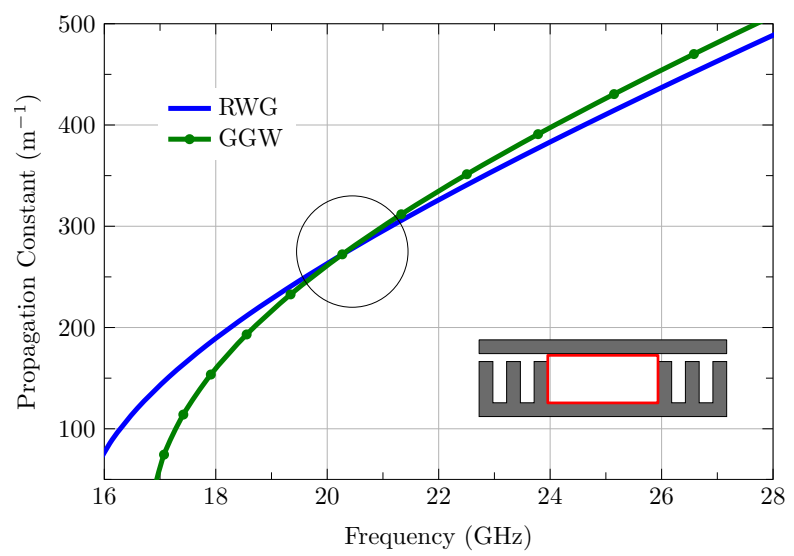

Figure 2.15: Propagation constant of two waveguides with the same crosssection (red box in the inset): rectangular and groove gap waveguide [208].

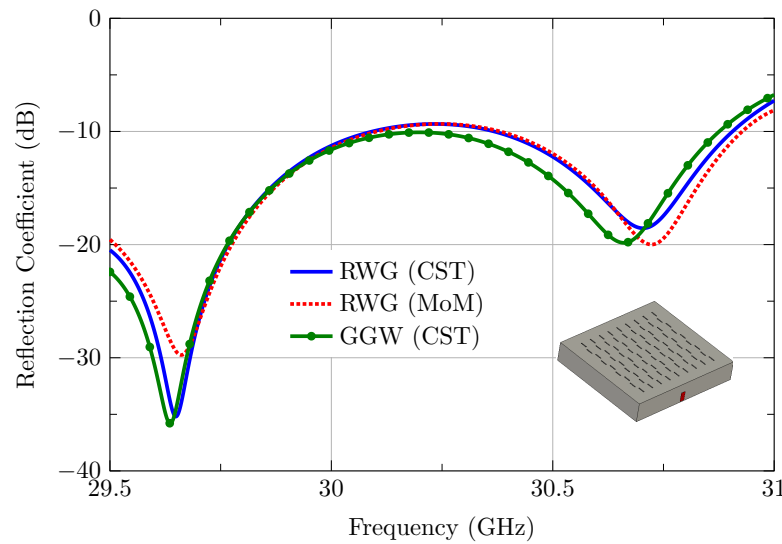

Figure 2.16: Reflection coefficient comparison for a subarray taken from the antenna presented in [208].

resembles to that of the equivalent RW. Thus, waveguide performance is not be significantly affected if an equivalent GGW replaces the RW. Fig. 2.17 shows a comparison of the magnetic field distribution between RW and GGW.

Finally, in [212] it was demonstrated the direct equivalent correspondence between the GGW and the rectangular waveguide. It was presented there a fast analysis method based on transmission line theory, which establishes the proper correspondence between the GGW and the RW. The authors concluded 


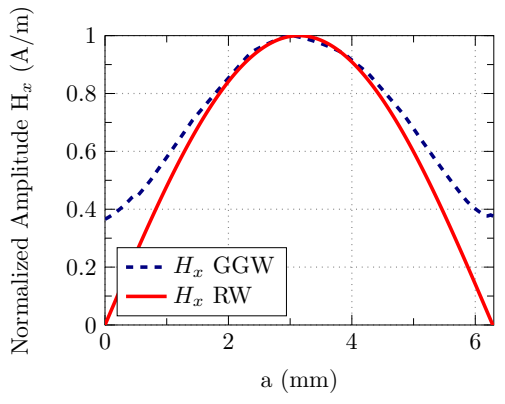

(a)

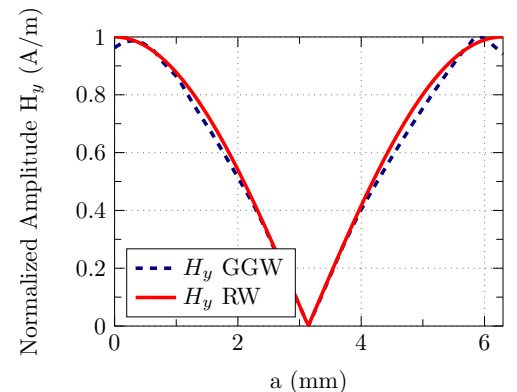

(b)

Figure 2.17: Normalized amplitude of the magnetic field of both waveguides [211]: (a) $H_{x}$ component and (b) $H_{y}$ component.

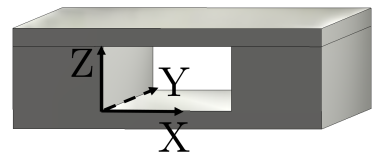

(a)

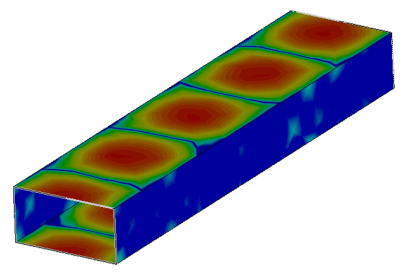

(c)

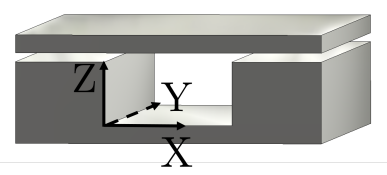

(b)

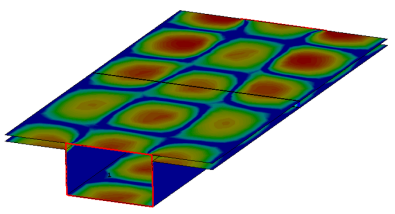

(d)

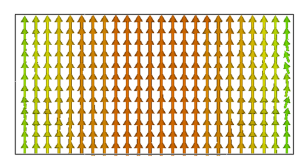

(e)

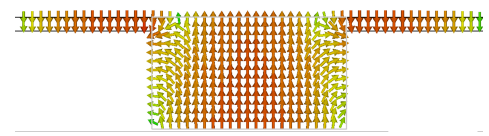

(f)

Figure 2.18: Magnetic field $H_{x}$ in a RW waveguide (c) perfectly sealed and (d) with an air gap of $0.5 \mathrm{~mm}$. Electric field $E_{z}$ in the RW (e) perfectly sealed and (f) with a gap of $0.5 \mathrm{~mm}$ between the metallic pieces. 


\section{CHAPTER 2. CORPORATE FEEDING NETWORKS BASED ON GAP WAVEGUIDE}

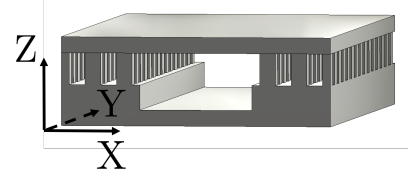

(a)

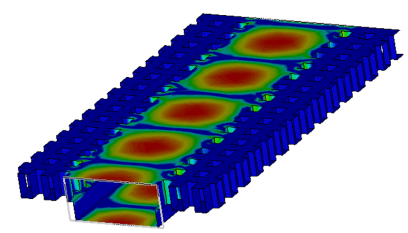

(c)

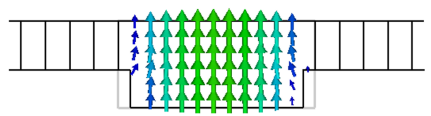

(e)

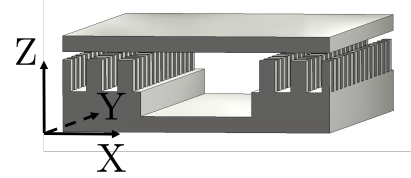

(b)

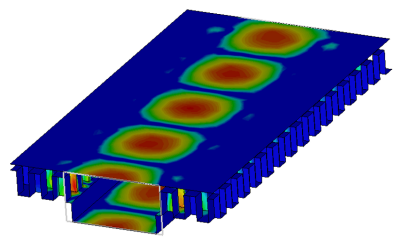

(d)

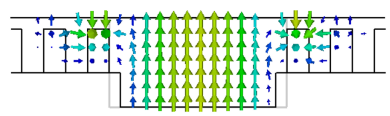

(f)

Figure 2.19: (a) GGW with zero-gap. (b) GGW with $h_{a}=0.5 \mathrm{~mm}$. (c) Magnetic field $H_{x}$ in a zero-gap GGW waveguide and (d) with an air gap of $0.5 \mathrm{~mm}$. Electric field $E_{z}$ (e) in a zero-gap GGW waveguide and (f) with an air gap of $0.5 \mathrm{~mm}$.

that GGW shows a behavior very similar to the RW and thus, the GGW is a suitable alternative to the RW.

Keeping this in mind, a comparative example of the standard WR-28 using RW and a GGW it is now taken as a benchmark. First, a perfectly sealed RW is used and then a gap of air is added below the top plate. Next, the same is done when using a GGW. The dimensions of WR-28 are $7.112 \mathrm{~mm} \times 3.556 \mathrm{~mm}$. GGW parameters are $w_{p}=1 \mathrm{~mm}, h_{p}=2 \mathrm{~mm}$ and $p=2 \mathrm{~mm}$. The air gap is $0 \mathrm{~mm}$ and $0.5 \mathrm{~mm}$ depending on the case.

As it is well-known, one aspect of particular importance for RWs is the field leakage across the pieces joints when they are not completely flat. Even a minimum air gap can greatly spoil the performance of any device. Some simulations will be shown for demonstrative purposes. The first example keeps a perfect electrical contact between the metal plates (Fig. 2.18a) and the second leaves an air gap of $0.5 \mathrm{~mm}$ (Fig. 2.18b). This air gap is determinant, as can be seen in Fig. 2.18d, since the field spreads through that tiny gap, degrading the expected behavior of a conventional waveguide. 
In the second case, a GGW is used (Fig. 2.19). The results are certainly enlightening. The magnetic field in both structures, without and with the gap, remains practically unchanged. It is observed how the blue zone (absence of $\mathrm{H}$-field) extends just up to the middle of the first nail even if there is a gap between the nails and the metal lid. On the other hand, an intense electric field is observed between the first nail and the upper metal plate. Then, the field is drastically attenuated around the second nail and it is already non-existent at the third nail. This demonstrates that, as a general rule, one row of nails is not enough to isolate completely two adjacent GGW branches but two rows could be enough depending on the design requirements. In any case, three rows would isolate them perfectly.

\subsection{GGW Corporate-feed networks}

Power dividers are building blocks of many microwave and millimeter-wave components and networks. Especially in microwave and millimeter-wave integrated circuits, the feeding system of an array antenna needs power dividers with low loss and broad bandwidth. Conventional rectangular waveguide power dividers can meet these requirements. For instance, power dividers fabricated from traditional waveguides have the advantages of low insertion loss and high power handling capability, but their large size and high cost is a limiting factor [213]. The aim of the following section is to apply the groove gap waveguide (GGW) technology to design power dividers to be used in feeding networks of large slot antenna arrays. To date, some array antennas have been realized based on groove gap waveguide distribution networks. Firstly, a H-plane coupling layer is presented. Then, examples of H-plane power dividers are described. Later an E-plane version will be shown, to end with the authors' proposal to improve these E-plane power-dividers to feed arrays in a more compact way.

\subsubsection{H-plane coupling layer}

A H-plane coupling layer using GW is presented in [208], within the LOCOMO project. It is part of a multilayer array antenna designed and fabricated for a SATCOM on-the-move terminal.

LOCOMO (LOw-cost and COmpact Ka-band MObile Satcom terminal) was a project carried out between 2013 and 2016, funded by the European Space Agency to develop precisely a SatCom on the move antenna in the Kaband. In the project were involved French companies (Thales Alenia Space, Vectrawave, IXBlue), the Spanish TTI, as well as the UPV, responsible for the complete design and manufacture of the antenna subsystem. The system was intended for dual use, civil and military, and therefore the reception band 


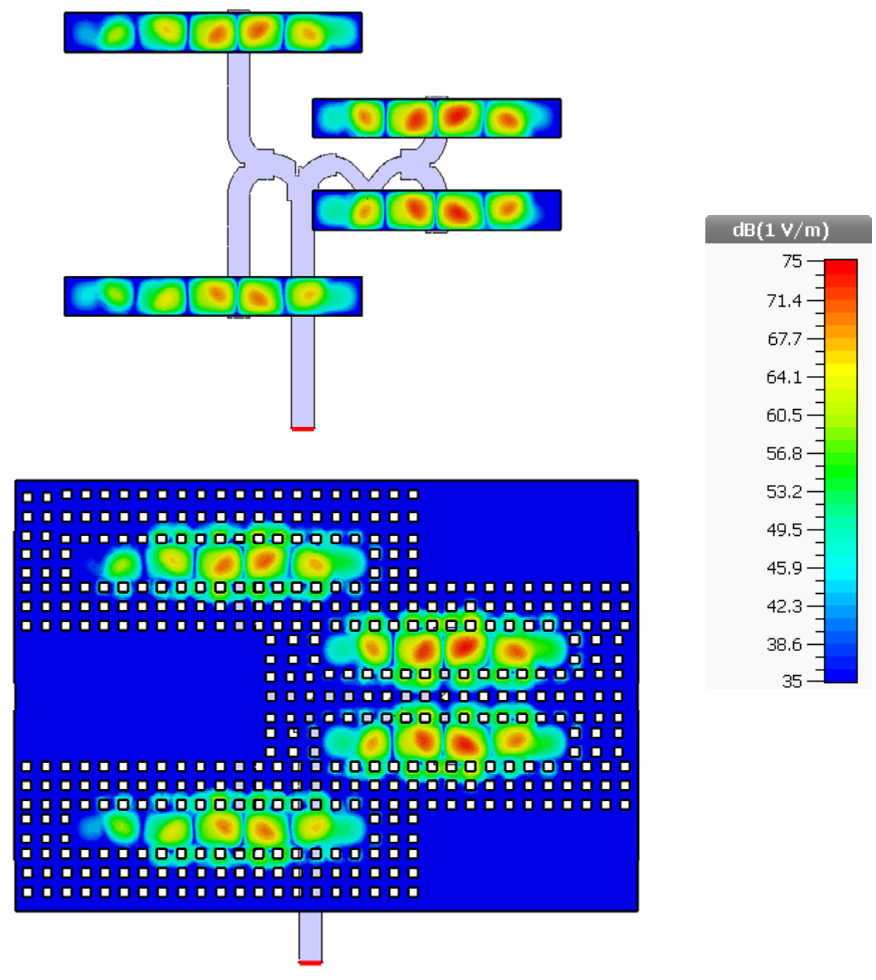

Figure 2.20: Electric field amplitude distribution (dB) in a conventional rectangular waveguide (above) and Groove Gap waveguide (below) [214].

covered from 19.7 to $21.2 \mathrm{GHz}$, while the transmission range was between 29.5 and $31 \mathrm{GHz}$. The antenna consisted of four radiating panels: two for TX, with right and left circular polarization and two others for RX. The feed network has been implemented using a combination of E-plane splitters coupled to H-plane waveguides.

A subarray layout of the feeding network is depicted in Fig. 2.20. The first distribution layer corresponds to 1 to 4 power dividers in the E-plane. An offcentered septum provides the required tailored power distribution as well as a wideband return-loss performance.

As shown in Fig. 2.20, Gap Waveguide technology was integrated into the intermediate layer of this power divider. In order to avoid leaks between the metallic layers that compose each panel, RW (upper figure) were replaced by nails (bottom figure). The qualitative view of the electric field distribution 


\subsection{GGW Corporate-feed networks}
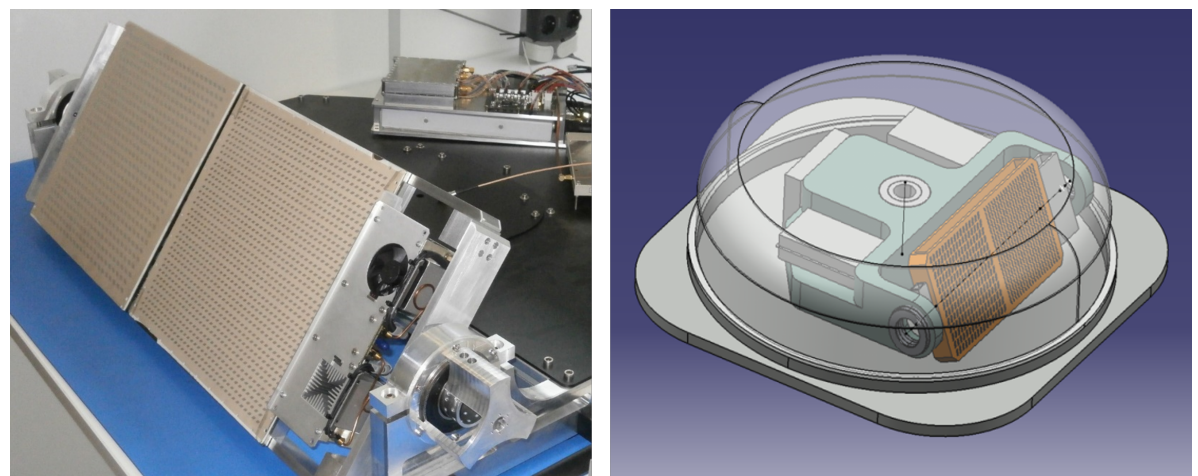

Figure 2.21: LOCOMO antenna mounted on rotatable axis. Two antenna subpanels for transmit and receive are visible. Two more are on the backside [214].

within the coupling waveguides shows good agreement between both. In addition, it is evidenced that the field remains confined in the groove.

Besides, LOCOMO gave the idea of designing E-plane splitters using Groove Gap Waveguide, which, in the end, proved to be a good alternative to get low-profile antennas in a single layer architecture. This project, which was a prior work, is standing outside the main scope of this thesis. Anyhow, a good summary of the complete system (Fig. 2.21), as well as the final results obtained can be consulted in [208].

\subsubsection{H-plane feeding networks in GGW}

There are few examples of H-plane feeding networks in GGW. Most GW networks used to feed array antennas are implemented with RGW or microstrip RGW [215]-[217]. The most common reason for not using corporate networks in GGW is their larger size compared with RGW-based ones.

One of the most recent examples of GW arrays fed by a H-plane feeding network was presented in [67]. The fundamental building block is a T-junction covering the V-band ( $50 \mathrm{GHz}$ to $70 \mathrm{GHz}$ ). The whole network is a 64 -way GGW power divider used to feed a high-gain and high-efficiency slot array antenna. The configuration of the T-junction is illustrated in Fig. 2.22. The topology provides in-phase power division with symmetrical amplitude distribution. As shown in Fig. 2.22, this power divider makes use of several nails to match the input port and split the incoming power from the input port to the output ones. Two different pin heights (A and B) are used as network tuning elements. The height of type-B nails is kept the same as that of all the other nails but the height of type-A nails is lowered. In order to satisfy the matching requirements 


\section{CHAPTER 2. CORPORATE FEEDING NETWORKS BASED ON GAP WAVEGUIDE}

over the desired frequency band, the position and height of the matching nails are optimized.

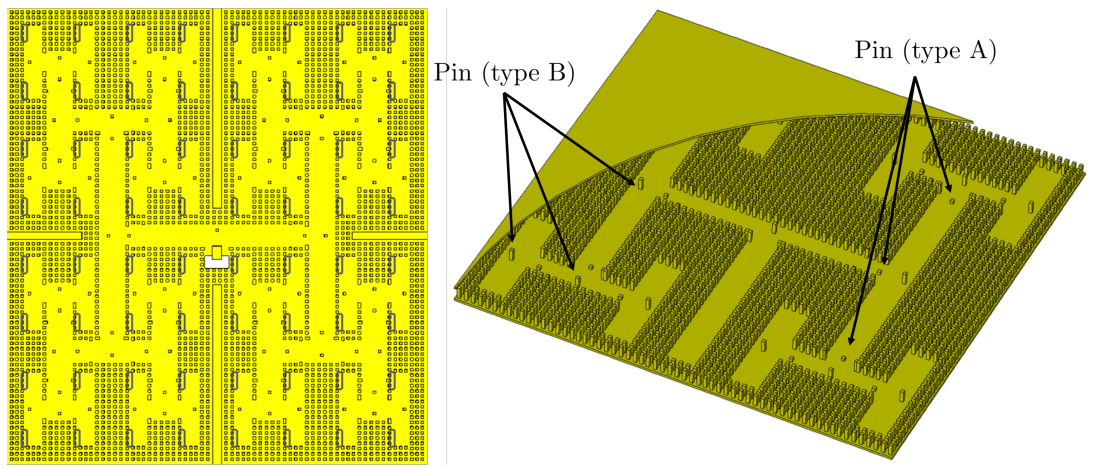

Figure 2.22: (a) Top view of the 64-way H-shaped GGW power divider and (b) 16-way one [67].
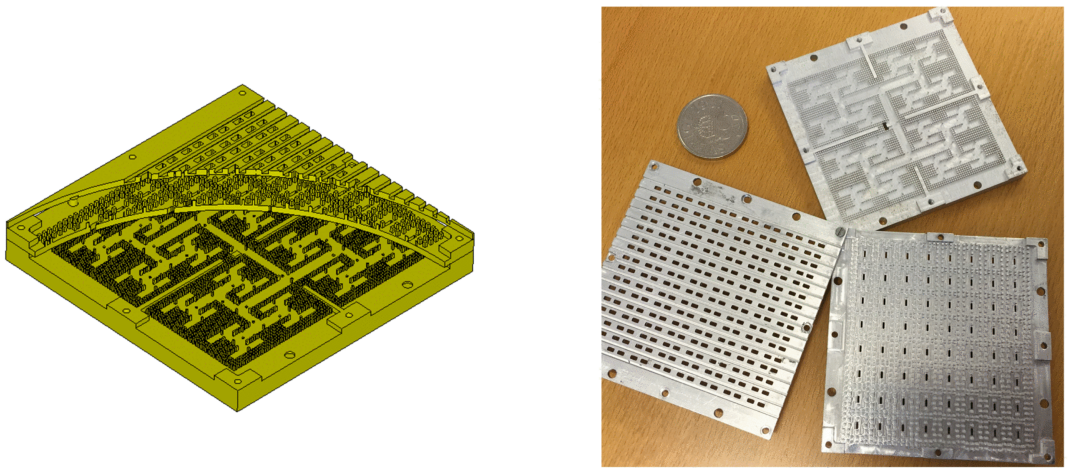

Figure 2.23: (a) Structure of the antenna array with $16 \times 16$ slots. (b) Manufactured antenna photograph. The dimensions of the antenna are $80 \times 80 \times 12.2 \mathrm{~mm}[67]$.

The total number of tuning nails in each 1-to-2 divider are three (type A) and three (type B) for achieving good performance over the desired frequency band. The overall performance of the antenna and experimental measurements can be checked in the aforementioned paper. The manufactured antenna, as well as each one of the constitutive layers, is shown in Fig. 2.23. Despite this solution is certainly satisfactory, it still presents some drawbacks. Firstly, the feeding network needs several layers to couple to the radiating layer, complicating the manufacturing and assembly process as well as increasing the total 


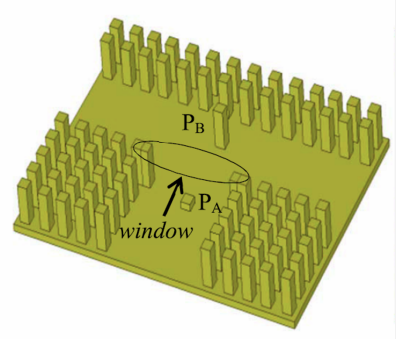

H-plane Power Divider

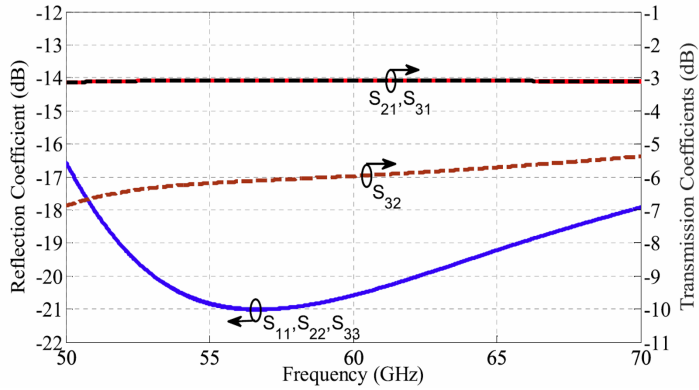

Figure 2.24: (Left) H-plane power divider of [67]. (Right) S-parameters.

antenna height. Besides, the height of the tuning nails (type B) may be a manufacturing challenge since it would be complicated to ensure the same height in every nail. This fact would eventually lead to unwanted imbalances on the output branches.

\subsubsection{E-plane feeding networks in GGW}

As a result of the above, an E-plane corporate feeding network is proposed here. The horizontally-polarized GGW is illustrated in Fig. 2.25. Placing the GGW vertically allows the branches of the distribution network to be closer to each other. Note that one of the ultimate goals when designing a feeding network is to enable arrays with close enough radiating elements.
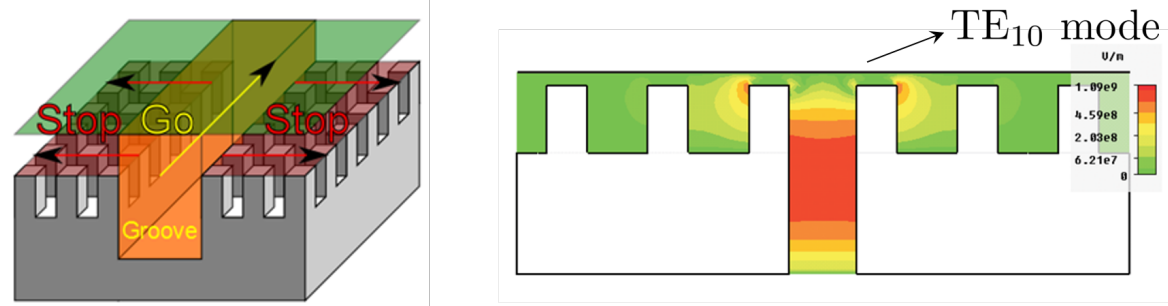

Figure 2.25: Narrow-wall GGW.

H-plane and E-plane GGWs use different tuning elements to achieve a good power balance between the ports of the divider, as shown in Figs 2.24 and 2.26. In the H-plane case, nails of different heights were used in the T-joint. In the E-plane case, two dividers are shown. The upper instance of Fig. 2.26 uses a step discontinuity, being width and length of the box the tunable parameters. 


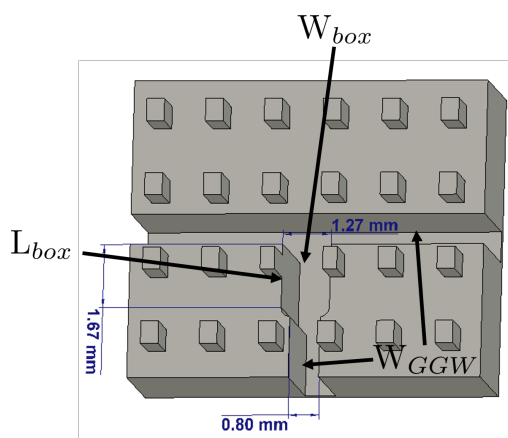

E-plane Power Dividers

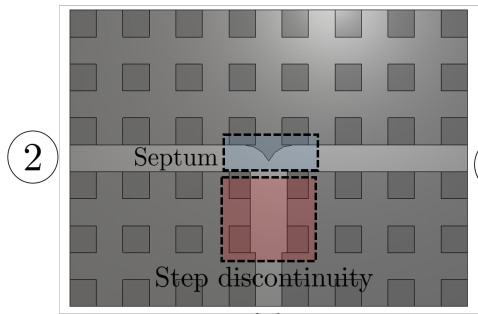

(1)
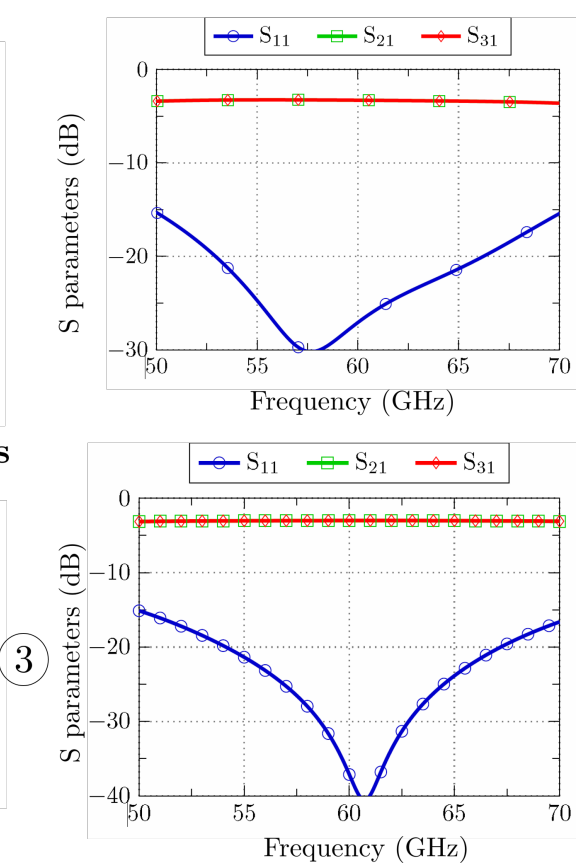

Figure 2.26: (Left) Proposed E-plane power dividers and (right) S-parameters obtained with each one.
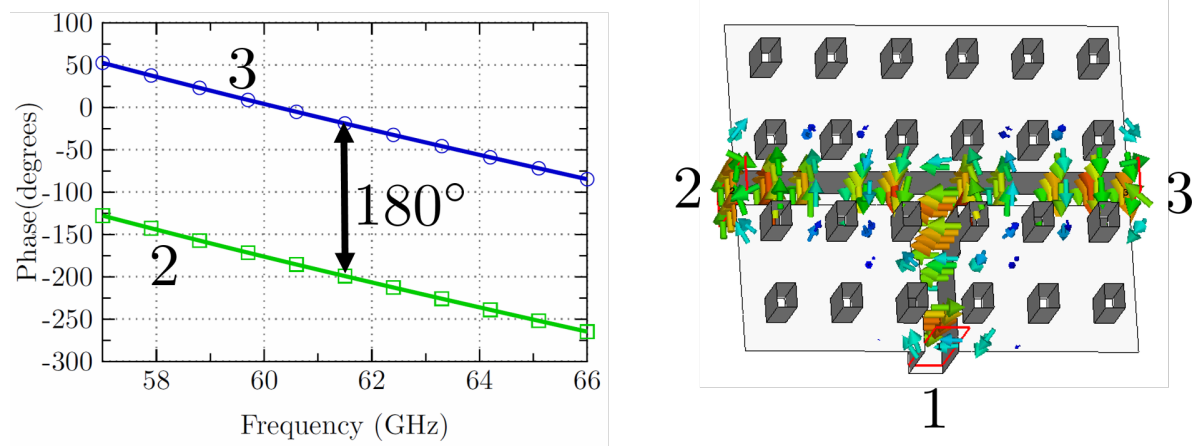

Figure 2.27: Behavior of the phase of the electric field in an E-plane power divider.

The second divider (bottom figure) uses also a septum to improve the overall 
matching. Both dividers are valid solutions since in both cases the reflection coefficient is lower than $-15 \mathrm{~dB}$ within a relative bandwidth larger than $30 \%$.
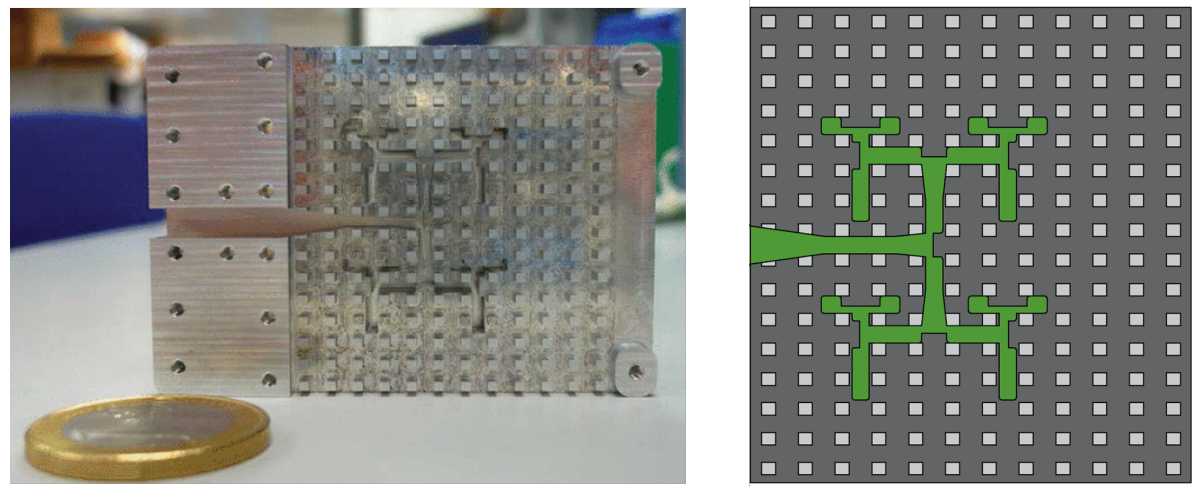

Figure 2.28: Fabricated $4 \times 4$ Ka-band array [175]: (a) without the cover to reveal the network and (b) layout of the corporate-feed network.
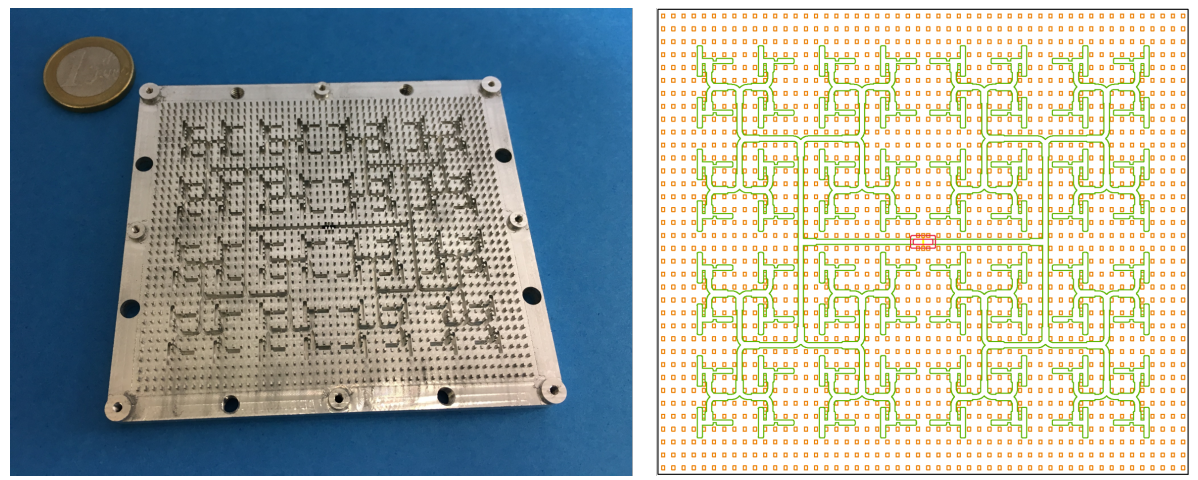

Figure 2.29: Fabricated $16 \times 16 \mathrm{~V}$-band array: (a) without the cover to reveal the network and (b) layout of the corporate-feed network.

However, the problem arising when using power dividers with horizontally polarized GGW is the well-known $180^{\circ}$ phase difference on output branches. Fig. 2.27 shows the behavior of the electric field where that $180^{\circ}$ phase hop is clearly seen between both ports. Conversely, the figure demonstrates that the field amplitude is divided equally between both arms thanks to the device symmetry. Nonetheless, it is possible to design E-plane distribution networks in GGW as long as a mechanism is conceived to fix this phase difference. 


\section{CHAPTER 2. CORPORATE FEEDING NETWORKS BASED ON GAP WAVEGUIDE}

The first antenna fed by an E-plane corporate network in GGW was presented in [175]. It is a small $4 \times 4$ Ka-band slot array which uses a GGW feeding network with asymmetrical arms. The final manufactured feeding layer and its layout can be seen in Fig. 2.28. It has also proved to be a valid solution in larger arrays, as shown in Fig. 2.29. It can be observed that branches in the left part are mirrored versions of those in the right part to counteract the $180^{\circ}$ phase shift. A detailed description of these prototypes is left for Chapter 3.

In [67], the H-plane feeding network required an intermediate layer to place the radiating elements close enough to avoid grating lobes. Comparing the dimensions of both networks, the E-plane network proposed here is $60 \%$ more compact than that H-plane version. On the other hand, while the height of the antenna is similar since placing the GGW vertically requires more height than horizontally, E-plane network is realized in one layer while H-plane requires two layers.

\subsection{RGW corporate-feed networks}

In the previous section two different ways to build distribution networks with GGW, E-plane and H-plane, have been explained. However, the most common all-metal distribution networks use RGW. On the one hand, RGW corporatefeed networks are more compact than vertically-polarized GGW. On the other hand, RGW avoids the above-mentioned phase problem in the power dividers.

One representative example of a typical network configuration in RGW is presented in [75]. There, a corporate-feed network for a $16 \times 16$-element slot array antenna is designed based on cascaded equally-split power dividers. Fig. 2.30 shows the configuration of the two-way ridge gap waveguide power divider as a building block of the full corporate-feed network.

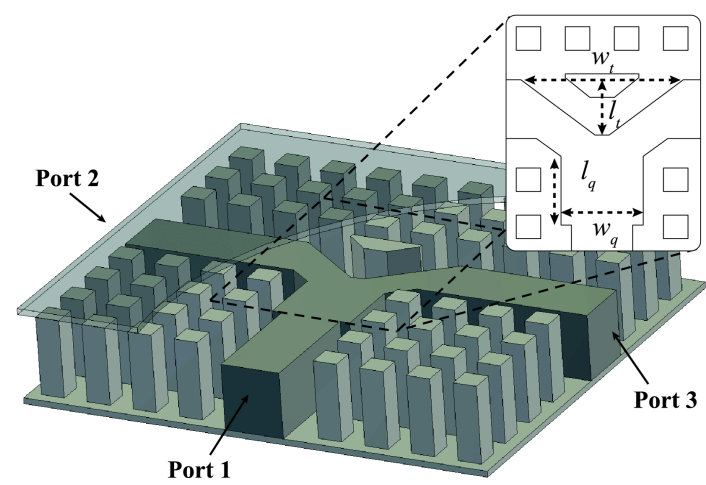

Figure 2.30: Geometry of the ridge gap waveguide power divider [75]. 


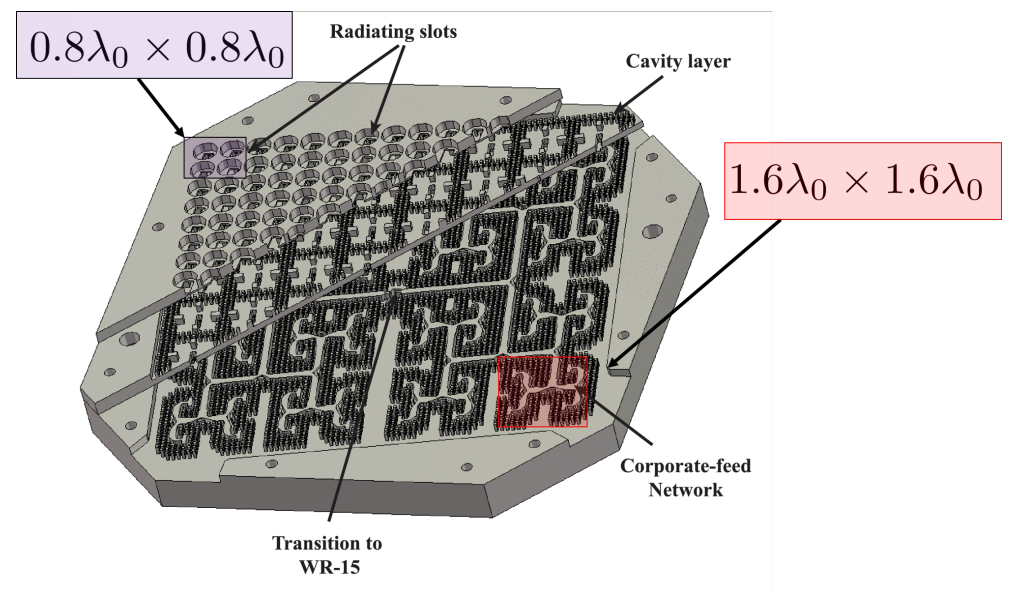

Figure 2.31: Full 1-to-64 corporate-feed network in RGW [75].

A quarter-wavelength matching box, as well as a gradual tapering from low to high impedance, are used to design a wideband power divider. The simulated performance of the power divider shows an input reflection coefficient below $20 \mathrm{~dB}$ with balanced amplitude and phase outputs over a frequency band from 55 to $75 \mathrm{GHz}$.

In this case, the ridge lines are separated by three nail rows, except near the T-shaped ridges and the coupling slots. Due to the limited room, the ridge lines are placed very closely in some places and therefore it could cause coupling between them. This effect has been seen and explained previously in Fig. 2.19. Besides, in [75] it is also demonstrated that the coupling is lower than $-20 \mathrm{~dB}$ in case of one row of nails, lower than $-37 \mathrm{~dB}$ using two rows of nails and $-50 \mathrm{~dB}$ for three rows of nails in the desired frequency band (57-68 GHz).

As can be observed in Fig. 2.31, a chandelier-shaped network is implemented, so that all output ports have the same phase. The need to arrange the network in this way makes it difficult to build a more compact structure. In fact, each network termination is separated $1.6 \lambda$ on both axes (X and $\mathrm{Y}$ ). That is the reason why a second distribution network, also called coupling layer, is needed above to finally feed the radiators. The resulting architecture responds to a multilayer antenna very similar to the H-plane GGW version presented in the previous section.

In view of the complexity of the afore-presented corporate-feed network, new topologies were investigated. The same group of researchers published, very recently (late 2017), a much more compact RGW network [218]. In this publication, unfortunately, the critical aspects of the network are not detailed. 


\section{CHAPTER 2. CORPORATE FEEDING NETWORKS BASED ON GAP WAVEGUIDE}

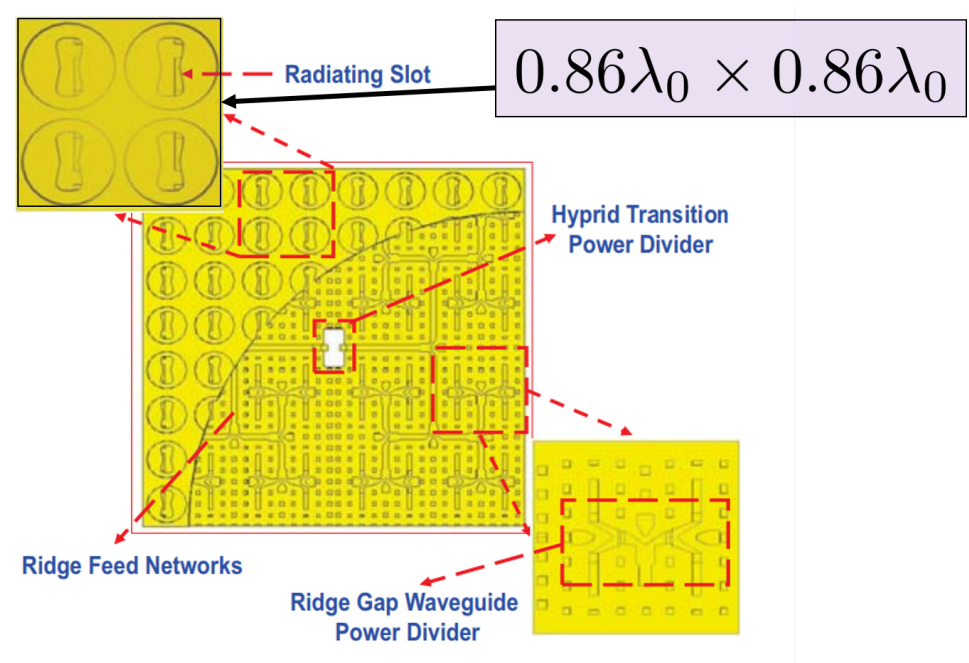

Figure 2.32: Schematic of the antenna presented in [218].

There, a RGW is capable of feeding, in one single layer, a two-dimensional array of $8 \times 8$ apertures in GW technology as depicted in Fig. 2.32. The chandeliertype corporate network is no longer used but a more compact H-shaped distribution network, avoiding the intermediate coupling layer. Radiating apertures are spaced $4.2 \mathrm{~mm}(0.86 \lambda)$ in both directions. It is a more compact solution than the previous one but, due to the lack of information in this short paper, it is difficult to assess and compare both designs.

As part of this work, and taking up the last developed GGW network (Fig. 2.29), other topologies are considered to address the $180^{\circ}$-phase-difference issue. Two possible alternatives have been implemented able to feed full-metal GW arrays: RGW and RGW-GGW networks.

In this context, as a contribution of the Thesis, an RGW power splitter was presented in [219]. The objective was to obtain a scalable network able to feed an array of large dimensions. A schematic drawing of the individual divider as well as the proposed architecture of an $8 \times 8$ array feeding network can be observed in Fig. 2.33. This figure is key to understand the problem with RGW networks and their need, in many cases, for intermediate coupling layers.

Although the unit cell is very compact $(0.93 \lambda \times 0.46 \lambda)$, the horizontal RGW branches force the apertures to be separated $0.46 \lambda$ and $0.73 \lambda$ alternatively. It can be concluded that the most limiting factor in the final array spacing involves the presence of the horizontal RGW sections.

Nonetheless, RGW networks are symmetric and more compact than those based on rectangular waveguides or vertically-polarized GGW. On the other 


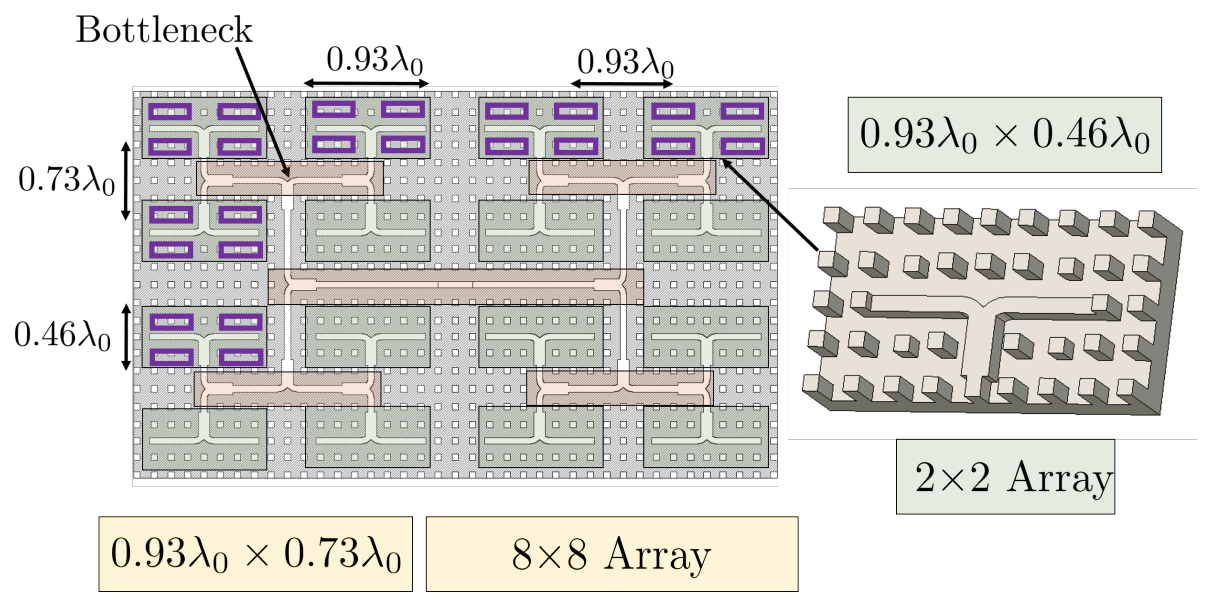

Figure 2.33: Schematic drawing with the cell presented in [219] and its integration in an $8 \times 8$ network.

hand, even more compact distribution networks would be possible using Eplane dividers, but the problem arises from the out-of-phase outputs of the splitters. The possibility to unify both types of networks in order to take advantage of their respective benefits will be addressed as the next step.

Table 2.1: Comparison of different proposals of RGW networks.

\begin{tabular}{|c|c|c|c|}
\hline Type & Spacing $\left(\lambda_{0}\right)$ & Layers & Band \\
\hline \hline Chandelier-shape [75] & $0.82 \times 0.82$ & $\mathbf{2}$ & $\mathrm{V}$ \\
\hline \hline H-shape [218] & $0.86 \times 0.86$ & 1 & $\mathrm{~V}$ \\
\hline \hline T-shape [219] & $0.93 \times 0.73$ & 1 & $\mathrm{~V}$ \\
\hline
\end{tabular}

\subsection{GGW-RGW corporate-feed networks}

As discussed above, E-plane dividers are able to provide very compact networks, but their inherent $180^{\circ}$ phase difference must be faced. A RGW network avoids such problem but more room is needed. An optimal solution combining both types of networks might hopefully lead to a compact in-phase approach. In order to verify the feasibility of such hybrid distribution networks, the first 

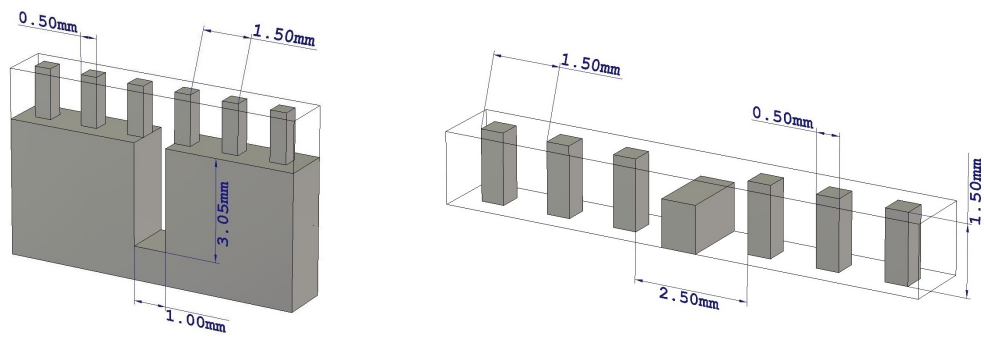

(a)

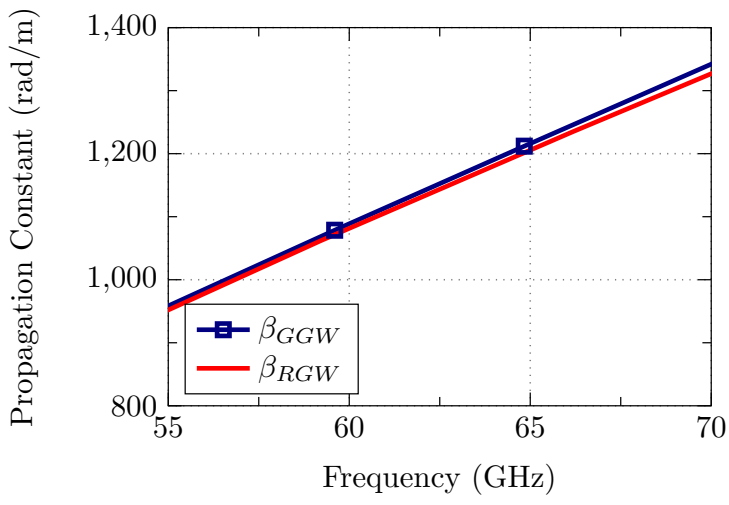

(b)

Figure 2.34: Basic dimensions of groove and ridge gap waveguides and (b) their respective propagation constant [220].

step is to demonstrate that ridge and groove waveguides are compatible and can be seamlessly combined.

For a certain height, width and periodicity of nails, the propagation constant is studied. Interestingly, an straightforward tuning of these parameters gives the same propagation constant in both waveguides. The basic dimensions of the groove and ridge gap waveguides are shown in Fig. 2.34a. This study was initially done for the V-band, though it can be easily extrapolated to any band by scaling the values. The propagation constant of RGW and GGW obtained with the dimensions shown in Fig. 2.34a is plotted in Fig. 2.34b. It is observed that perfect agreement between both, $\beta_{G G W}$ and $\beta_{R G W}$, can be achieved within the band of interest ( 57 to $66 \mathrm{GHz}$ ). The stopband of the bed of nails spans from $40 \mathrm{GHz}$ to $70 \mathrm{GHz}$. 


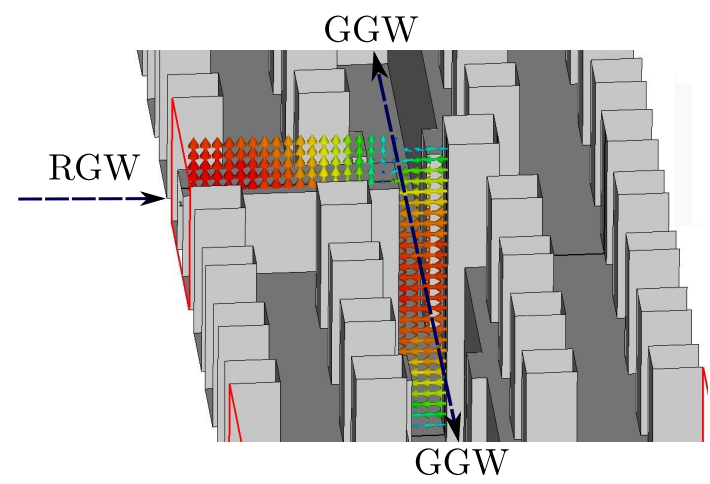

Figure 2.35: Electrical field representation at the discontinuity between RGW and GGW.

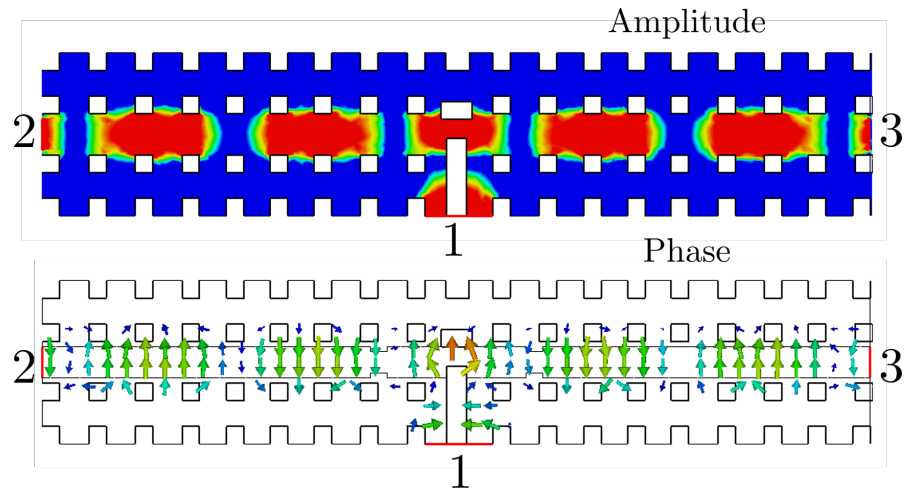

Figure 2.36: Amplitude and phase of a power divider combining GGW and RGW.

\section{Power splitter from RGW to GGW}

In order to couple the propagation modes from RGW to GGW, a power splitter has been designed. After several attempts the first conclusion was that the ridge must protrude a little bit into the GGW to be able to couple the field from the RGW. In Fig. 2.35, the behavior of the electric field and how it rotates from the RGW to the GGW can be observed. This good behavior of the field coupling has been crucial to achieve more compact hybrid networks, solving most of the drawbacks explained in previous designs.

Let us compare Fig. 2.27 with Fig. 2.36. E-plane divider is more compact in the horizontal plane since the GGW fits between two rows of nails. However, 


\section{CHAPTER 2. CORPORATE FEEDING NETWORKS BASED}

ON GAP WAVEGUIDE

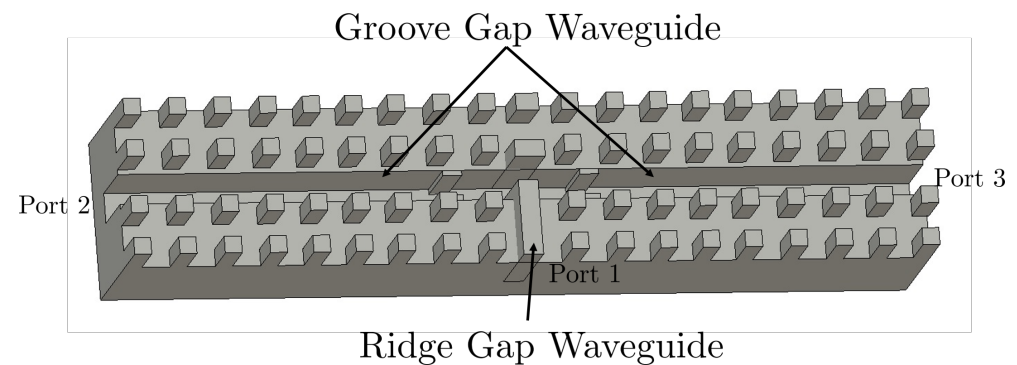

Figure 2.37: RGW to GGW transition.

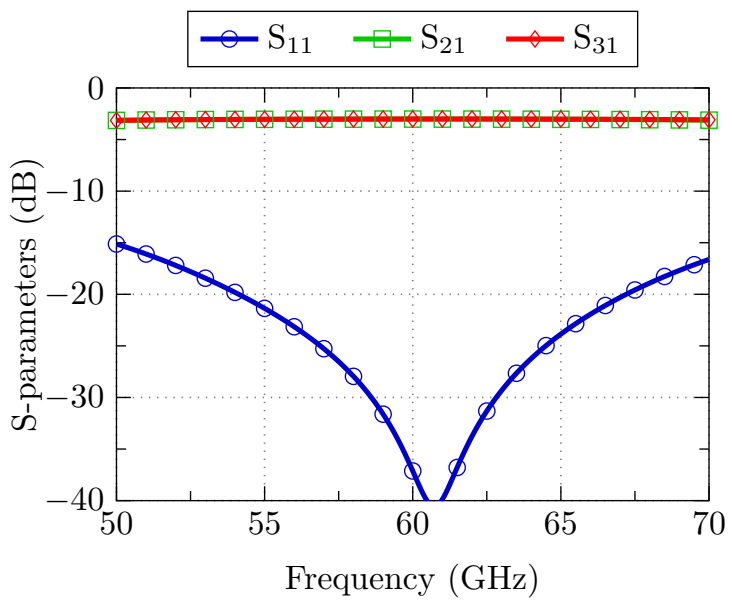

Figure 2.38: Simulated S-parameter magnitude for the RG transition.

this type of divider introduces a $180^{\circ}$ phase difference on both sides of the divider. Now, coupling the RGW to a GGW, the phase of all ports are equal without requiring to any asymmetry to fix this effect (Fig. 2.39). At the same time, this transition is a divider, since it splits the power from the RGW input port to both sides of the GGW. In Figs. 2.37 and 2.38 the divider and its Sparameters are shown. Despite this change of waveguide type, a remarkable $37 \%$ bandwidth for an $\mathrm{S}_{11}$ lower than $-10 \mathrm{~dB}$ is achieved.

\section{Design of a 1 to 4 power divider combining RGW to GGW}

A schematic drawing of a 1 to 4 power divider combining RGW and GGW is shown in Fig. 2.40. A central RGW (yellow block) is subsequently coupled to a GGW (blue block) which in turn is coupled to two RGWs on each side, 


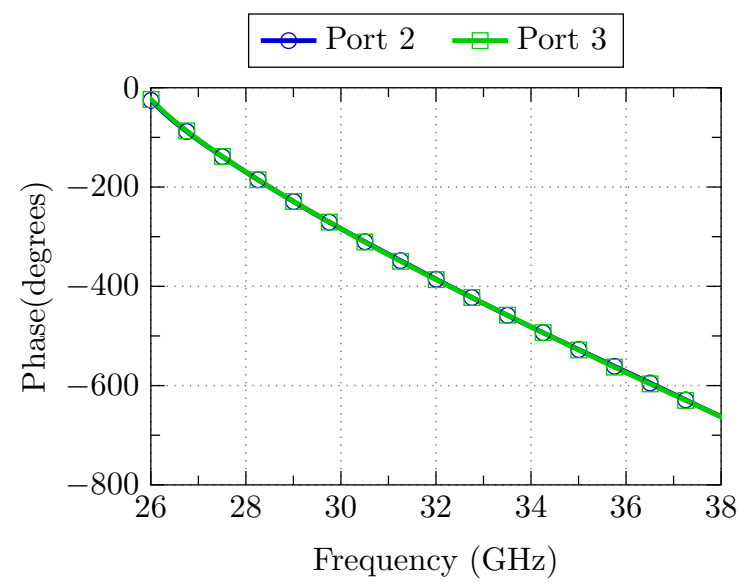

Figure 2.39: Simulated S-parameter phase for the output ports in the RG transition.

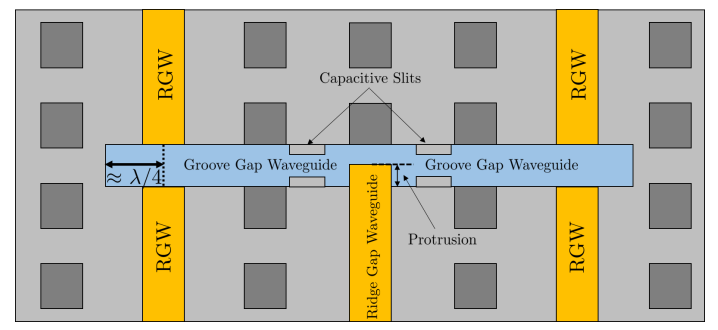

Figure 2.40: Schematic drawing of a 1 to 4 power divider combining RGW and GGW.

leading to a 1 to 4 power divider combining RGW and GGW. There are two fundamental parameters to be taken into account for a proper network design. A fundamental tuning parameter to match the network is the short-circuit distance of the GGW concerning the RGW position. This distance must be approximately $\lambda_{g} / 4$. In this way, E-field maximum occurs just at the input of the RGW for a proper power coupling. Moreover, capacitive slits can be included on both sides of the input RGW-GGW transition to improve the frequency bandwidth. Nonetheless, these slits are not an element as decisive as the tuning parameters mentioned above (protrusion and short-circuit).

In Fig. 2.41, the proposed power divider is shown. Fig. 2.42 shows the phase of the magnetic field when exciting the input port. It is observed that the four output ports have the same phase, just like in an all-RGW network. Fig. 2.43 


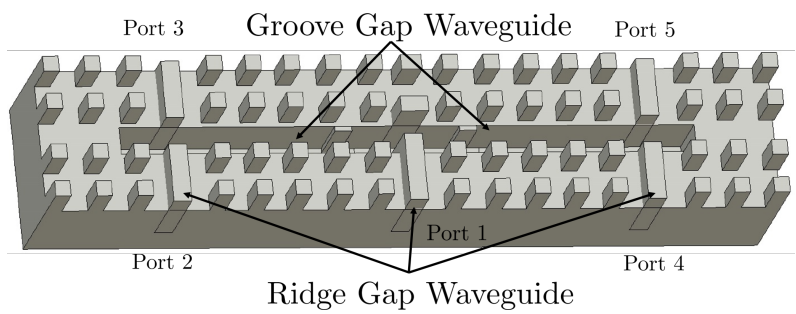

Figure 2.41: Combined ridge-groove-ridge divider (RGR).

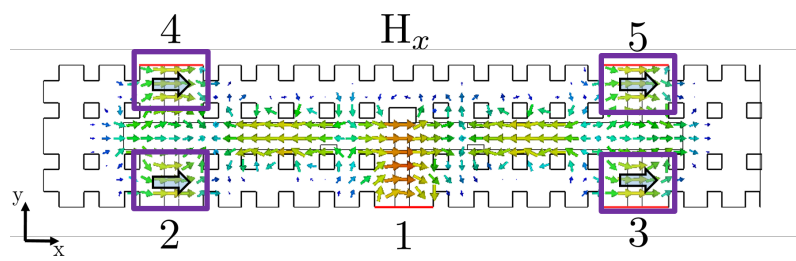

Figure 2.42: Magnetic field at the output ports of the 1 to 4 power divider.

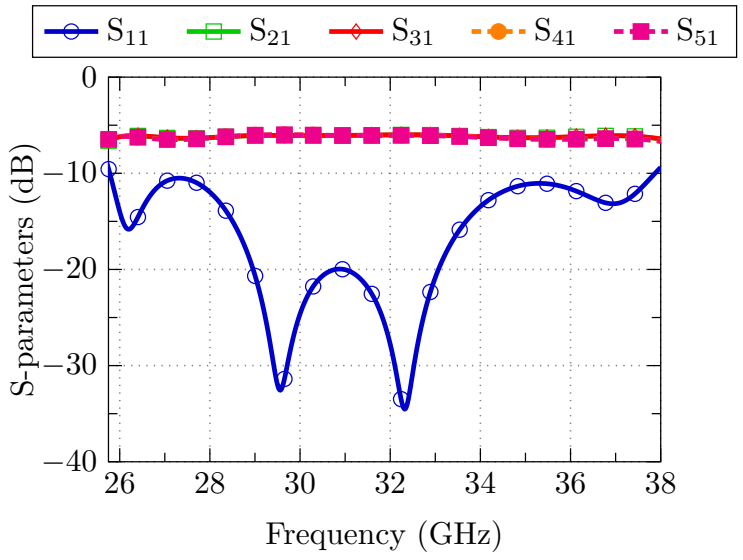

Figure 2.43: Simulated S-parameters of the RGR transition.

plots the simulated S-parameters versus frequency. The power is equally distributed to the output ports with a reflection coefficient lower than $-10 \mathrm{~dB}$ over a $37 \%$ frequency bandwidth. The RGW to GGW transition may help to specific corporate-feed networks that require a compact design. The primary advantage in combining these two types of waveguides is that they introduce a very profitable versatility in the design of corporate-feed networks. Whereas 


\subsection{GGW-RGW corporate-feed networks}

\begin{tabular}{cccc}
\hline & GGW & RGW & RG-GW \\
\hline Compact & $\checkmark$ & $\boldsymbol{x}$ & $\checkmark$ \\
Phase & $\boldsymbol{x}$ & $\checkmark$ & $\checkmark$ \\
\hline
\end{tabular}
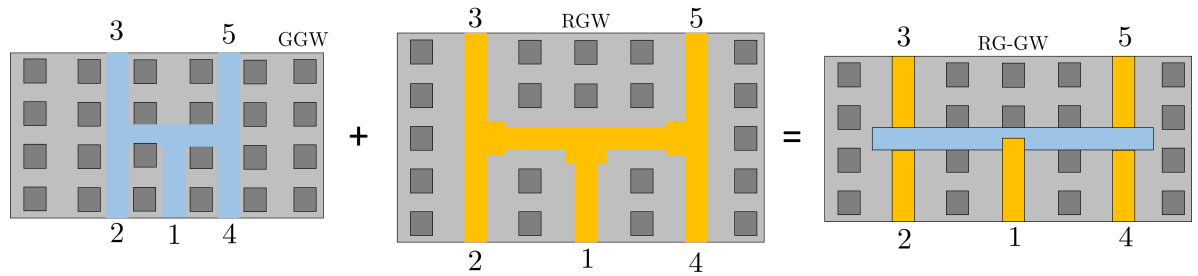

Figure 2.44: Evolution of the combination of a GGW network with a RGW network to obtain the hybrid network with both types of waveguides.

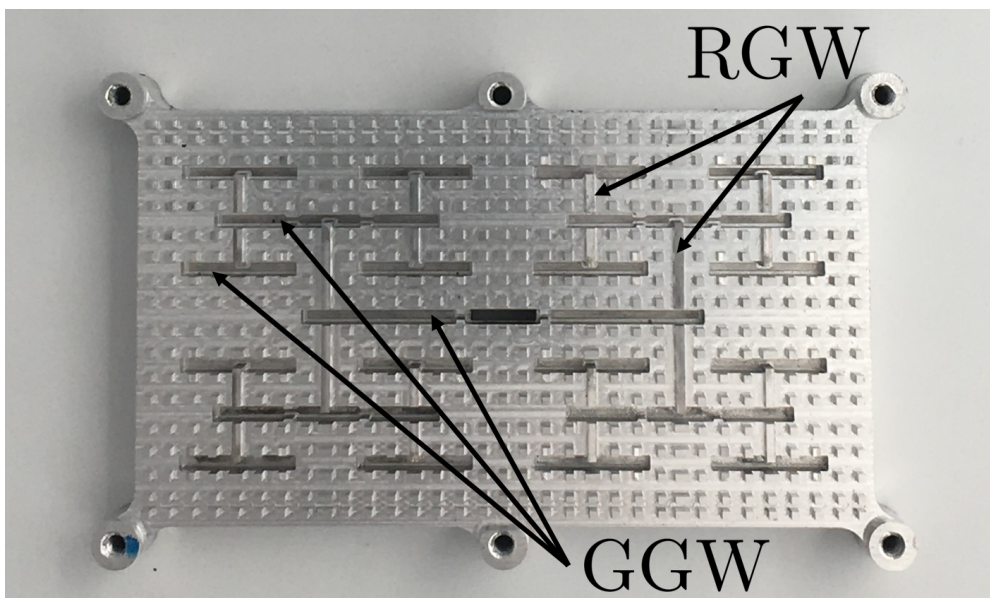

Figure 2.45: Fabricated ridge-groove feeding network.

RGW leads to symmetrical networks, GGW sections provide a more compact structure (Fig. 2.44). This hybrid network, one of the main outcomes of this Thesis, has been widely adopted in the arrays finally developed in Chapter 4 . As an example, Fig. 2.45 shows the combined ridge-groove network integrated into a manufactured antenna. 
CHAPTER 2. CORPORATE FEEDING NETWORKS BASED ON GAP WAVEGUIDE 


\section{Chapter 3}

\section{Radiating elements for Gap Waveguide Arrays}

"I believe any success in life is made by going into an area with a blind and furious optimism."

— Sylvester Stallone

The present chapter addresses the use of apertures, whether rectangular or circular, as radiating elements for GW arrays. These radiating elements are fed by the GW networks described in the previous Chapter 2. Rectangular apertures are cavity-backed by coaxial cavities while the circular apertures are fed by cylindrical cavities. However, to have a global understanding of the subject to be discussed, the fundamental principles of radiation of apertures are reviewed first.

The concept of radiating apertures was already known more than 300 years ago, as evidenced by the work carried out by Huygens in the late 17th century. Paradoxically, it was not until the mid-twentieth century, with the World War II that due attention was paid to this concept [221]. After the WWII and in later years, Watson [222] and Stevenson [223], proposed for the first time to use slots on the walls of a rectangular waveguide to couple and radiate energy. Later, Orefice and Elliot [224] proved they could control antenna parameters like external mutual coupling, input impedance, and radiation pattern by adjusting the length, spacing and orientation of the slot elements within a rectangular waveguide array. It was then that the basic concepts of slot waveguide arrays were established. Even today it remains a widely used solution because of its easy implementation and low cost, especially in the microwave band. 


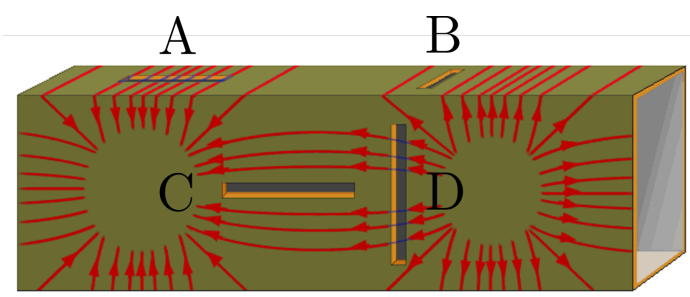

Figure 3.1: Some slot arrangements in a rectangular waveguide.

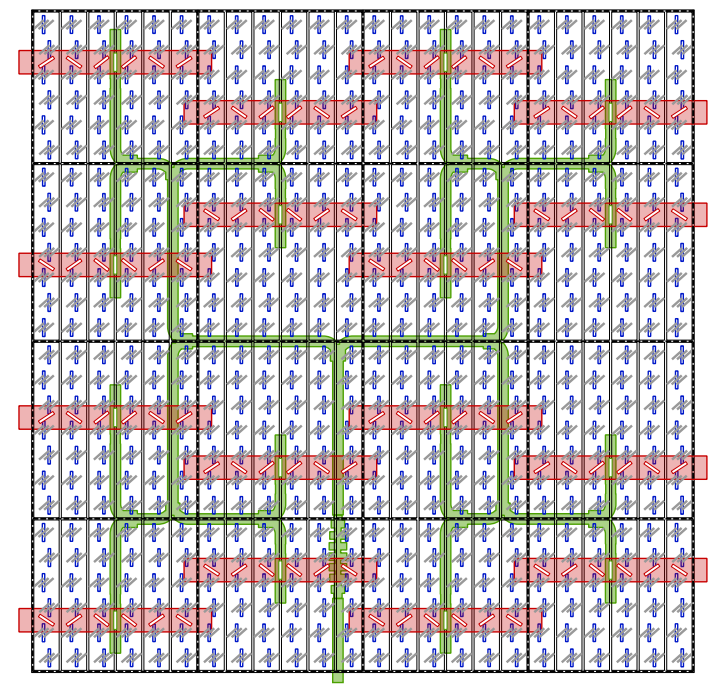

Figure 3.2: LOCOMO project. Layout of receive antenna comprising $4 \times 4$ subarrays. It shows parasitic dipoles (grey), radiating slots (blue), radiating waveguides (black) and coupling waveguides (red). Feed network is shown in green.

Slotted Waveguide Antennas (SWA) radiate energy through slots cut in a broad or narrow wall of a rectangular waveguide. These slots introduce discontinuities in the conductor and interrupt the flow of current along the waveguide. Hence, the current must flow around the edges of the slots, causing them to act as dipole antennas. The position, shape, and orientation of the slots will determine how (or if) they radiate. Fig. 3.1 shows a rectangular waveguide with a red-line schematic of the current distribution on the waveguide walls.

Among the main advantages of SWA are their relatively low weight and small volume, and their high power handling, high efficiency, and good match- 
ing properties. For this reason, SWA has been an ideal solution for many radar, communication, navigation, and high-power microwave applications. As mentioned in Chapter 2 the use of slotted waveguides was explored in the initial phases of the thesis for the implementation of a multilayer antenna (Fig. 3.2) for SATCOM applications [208].

\subsection{Slots fed by Coaxial Cavities}

A coaxial cavity resonator is a cavity structure resembling a finite-length coaxial transmission line which is closed at both ends and shorted at one end as illustrated in the Fig. 3.3. At the other end, a small gap is left, behaving as a capacitance between the inner and outer conductors. Typically, resonant coaxial cavities have been used for filter design [225]-[227], also known as combline filters. In this regard, the ability of gap waveguide technology to create high-Q resonators for waveguide cavity filters is also well documented [148]-[154]. In this case, thanks to the TEM nature of the coaxial cavity mode, its size can be freely reduced without worrying about the cutoff condition. In addition, it has been shown that coaxial cavities could also be a good alternative to feed slots, instead of using rectangular waveguides [175].

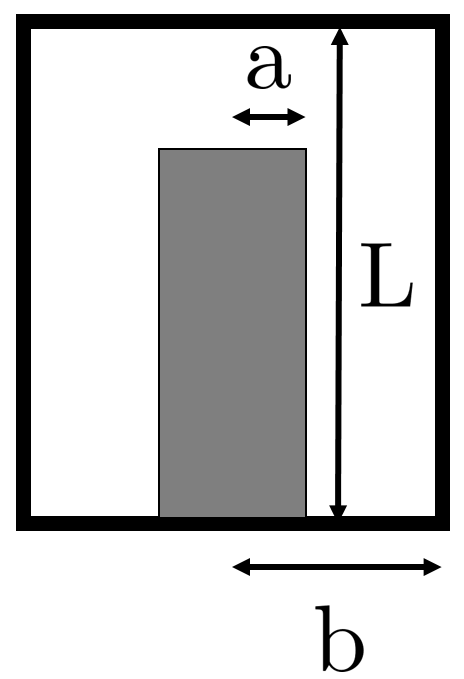

Figure 3.3: Coaxial cavity structure. 


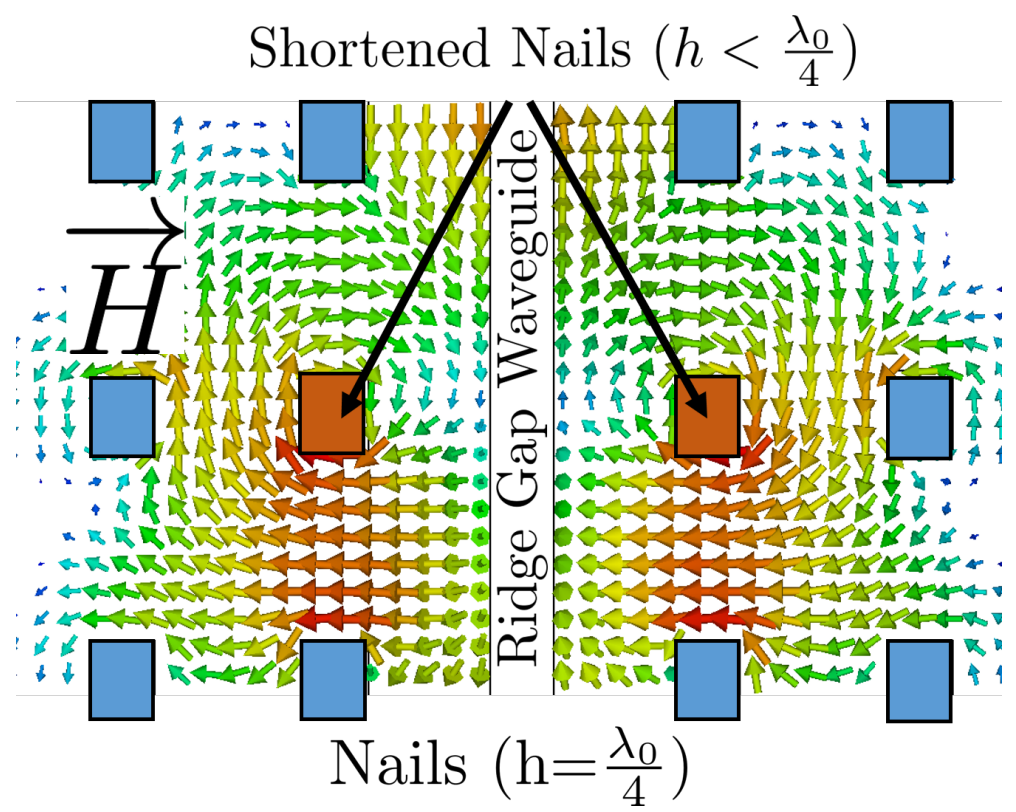

Figure 3.4: H-field distribution within gap waveguide coaxial cavities. The cavities are excited through a RGW.

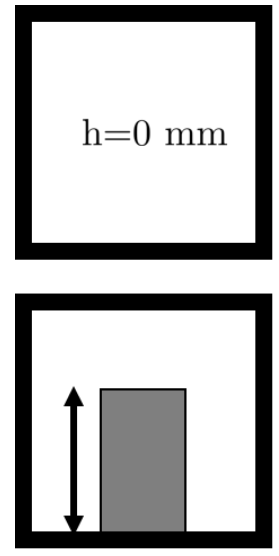

$\mathrm{h}=1.5 \mathrm{~mm}$
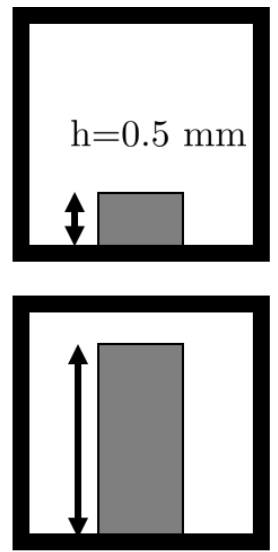

$\mathrm{h}=2 \mathrm{~mm}$
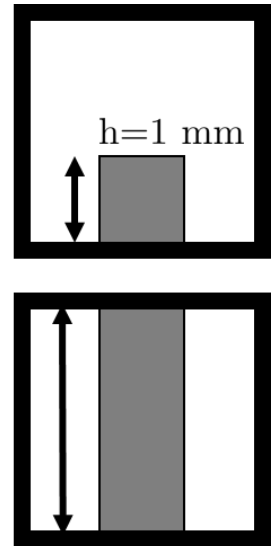

$\mathrm{h}=2.5 \mathrm{~mm}$

Figure 3.5: Different nail heights from $h=0 \mathrm{~mm}$ to $h=2.5 \mathrm{~mm}(\lambda / 4$ at $30 \mathrm{GHz})$ in steps of $0.5 \mathrm{~mm}$. 


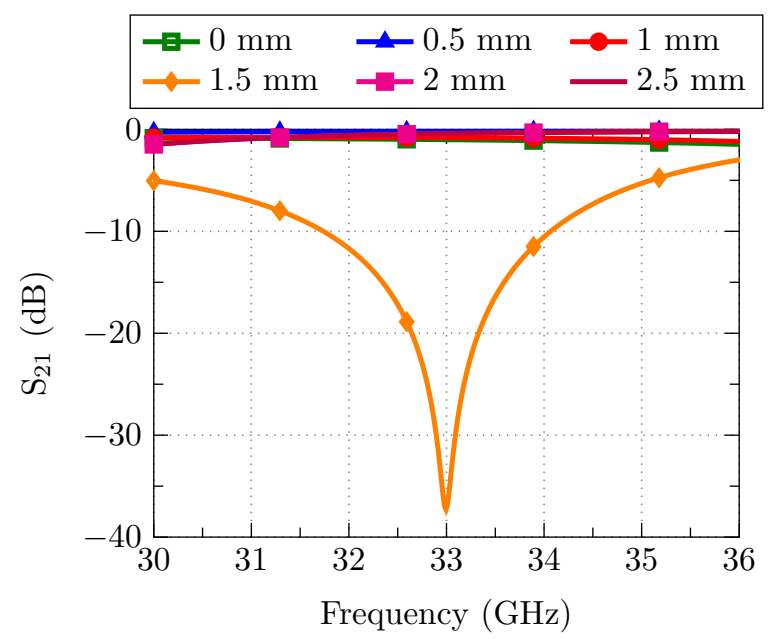

Figure 3.6: Transmission coefficient of the structure depicted in Fig. 3.4 for different nail heights.

\subsubsection{Coaxial Cavities in Gap Waveguide}

The typical feeding scheme of coaxial cavities in Gap Waveguide is presented in Fig. 3.4. It consists of a bed of nails where the height of two of them, drawn in orange, is reduced. The height of the rest of the nails is $\lambda / 4$. A parametric study of the shortened-nail height is performed from $h=0 \mathrm{~mm}$ to $h=2.5 \mathrm{~mm}$ (in steps of $0.5 \mathrm{~mm}$ ), as represented in Fig. 3.5. In Fig. 3.6 the transmission coefficient is shown. As it is observed, the coaxial cavity is resonant for $h=1.5 \mathrm{~mm}$. At the resonance frequency, the RGW properly excites the coaxial cavity mode. This fact can be seen in Fig. 3.4, where the typical loops of the magnetic field are created around the shortened nails.

Now, if a slot is conveniently placed over the resonator, parallel to the magnetic field, an efficient radiation is produced. The coaxial cavity provides a great versatility to locate the radiating apertures. From the behavior of the magnetic field, up to 4 possible slot arrangements can be defined for an inphase excitation (Fig. 3.7). The most symmetric options are (a) and (d) since they are equally separated from the feeding ridge. For options (b) and (c), though they are perfectly valid with regard to the H-field direction, slots would be unequally excited in amplitude.

In this example, the coaxial cavities are fed through a central ridge, but it can also be done through a groove, as shown in Fig. 3.8. In fact, this approach is more optimal since it allows to place closer the cavities exciting the radiating apertures above them. A similar behavior of the H-field in the cavities fed by 
(a)

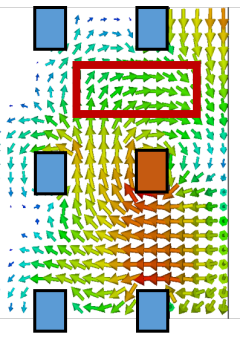

(c)

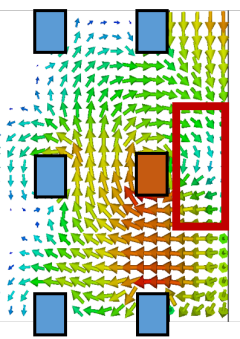

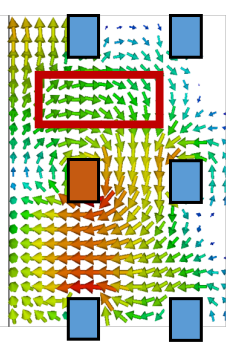

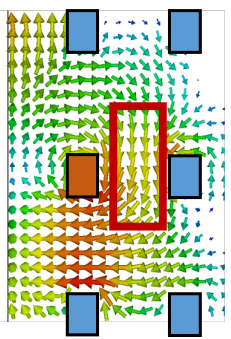

(b)
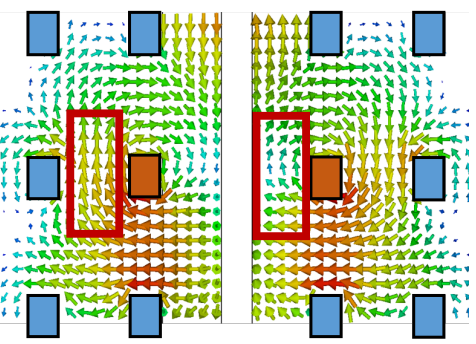

(d)
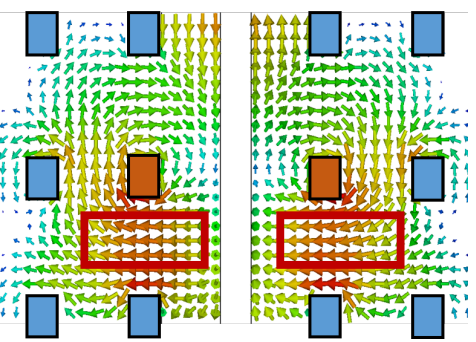

Figure 3.7: Different valid positions to place the apertures for an in-phase radiation.
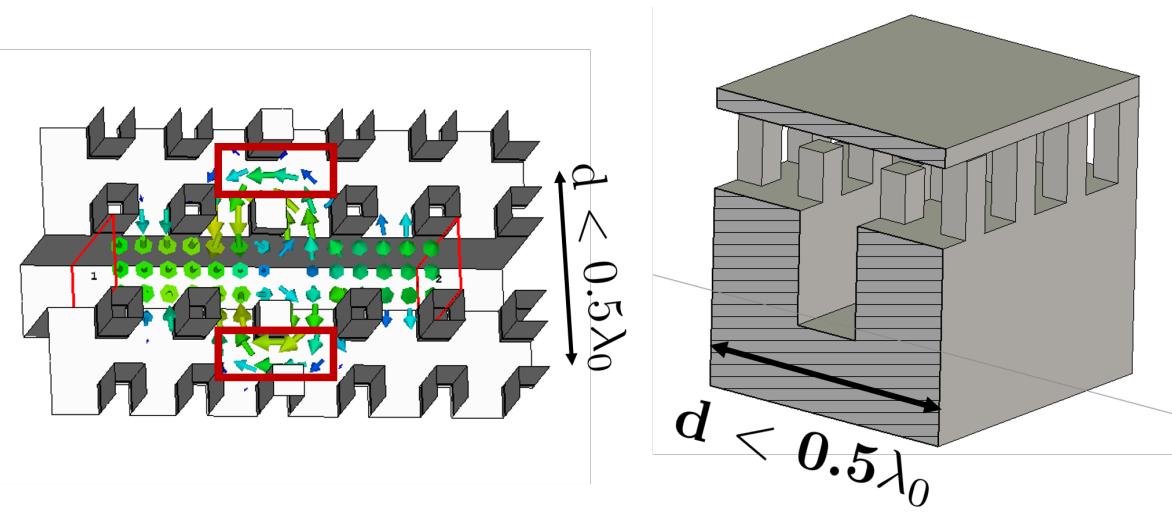

Figure 3.8: Coaxial cavities excited by a GGW.

a GGW is observed. Interestingly, distance between cavities can be less than $0.5 \lambda_{0}$. Since they are separated by the groove and the coaxial nails only, it is possible to bring the cavities closer by adjusting the nail periodicity and the groove width, if necessary. These first feeding examples demonstrates the versatility of the coaxial cavities excitation and their advantages in terms of compactness, a crucial property for array antenna design. 

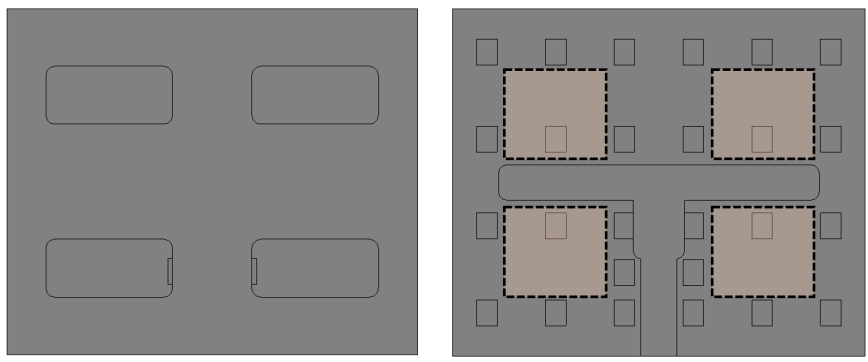

Figure 3.9: $2 \times 2$ Cavity-backed slot-antenna fed by a symmetric GGW.
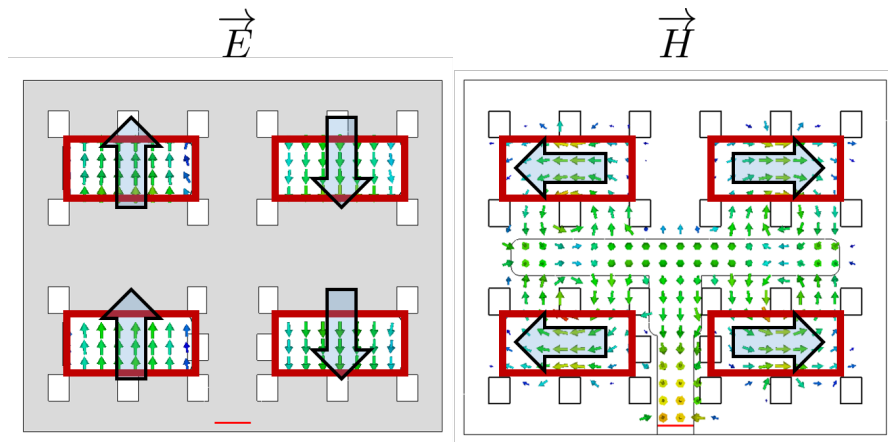

Figure 3.10: $\mathrm{E}$ and $\mathrm{H}$ field in a $2 \times 2$ cavity-backed slot array using a symmetric groove gap waveguide feeding.

\subsubsection{Groove Gap Waveguide feeding}

In the previous section, we have seen the basic structure of a coaxial cavity and how the magnetic field is confined around the shortened nail of the textured surface. It has also been seen that both the RGW and the GGW are able to excite these cavities. However, each option must be studied separately in order to understand their particularities. Intuitively, the GGW seems to be the best candidate since it is more compact and fits easily between two rows of nails. In addition, its horizontal polarization facilitates the field coupling to the shortened nail.

Nevertheless, as explained in Chapter 2, an E-plane 1:2 power splitter as the one depicted in Fig. 3.9 introduces a $180^{\circ}$ phase difference on each branch (Fig. 3.10). If this effect is not corrected, the cavities are excited with opposite phases on each side of the subarray. E and $\mathrm{H}$ fields of this subarray are shown 

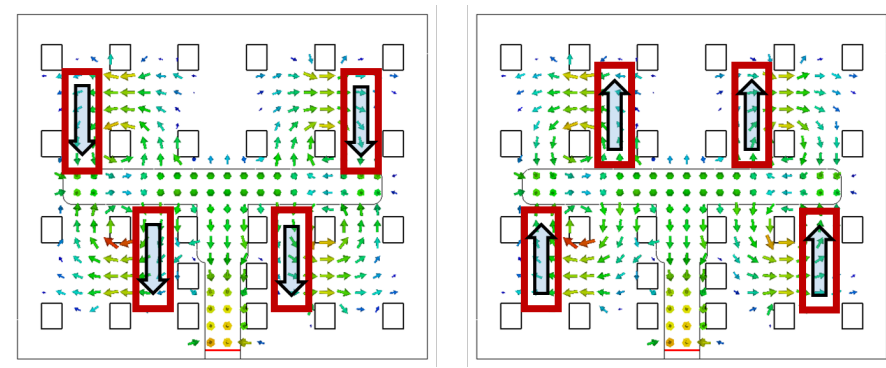

Figure 3.11: Location of the apertures for coherent radiation.
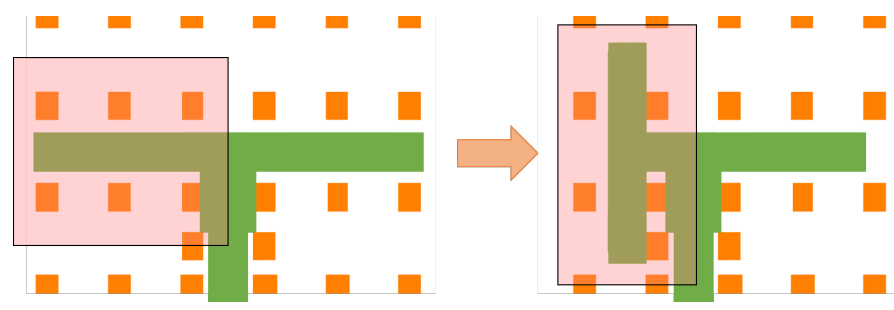

Figure 3.12: (Left) Symmetric GGW feeding. (Right) Asymmetric GGW feeding.

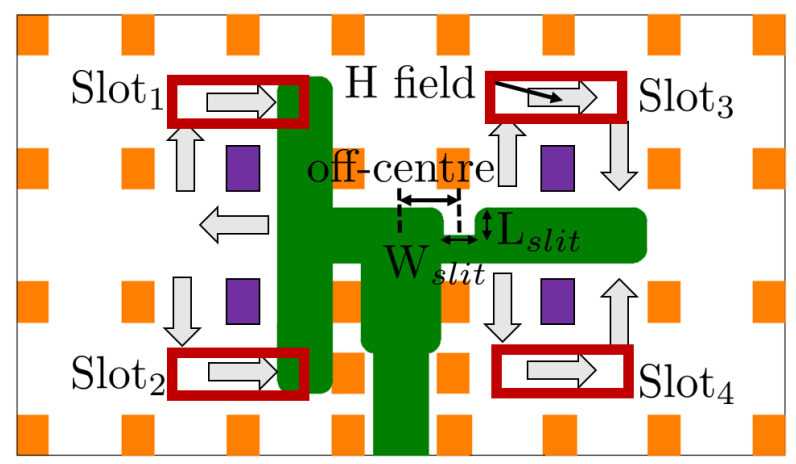

Figure 3.13: Unit cell of a $2 \times 2$ subarray fed by GGW. The most representative elements are shown: feeding network (green), magnetic field (arrows), shortened-nails (purple) and apertures (red).

in Fig. 3.10. However, Fig. 3.7 has demonstrated that there is some degree of versatility to place the radiating apertures. 


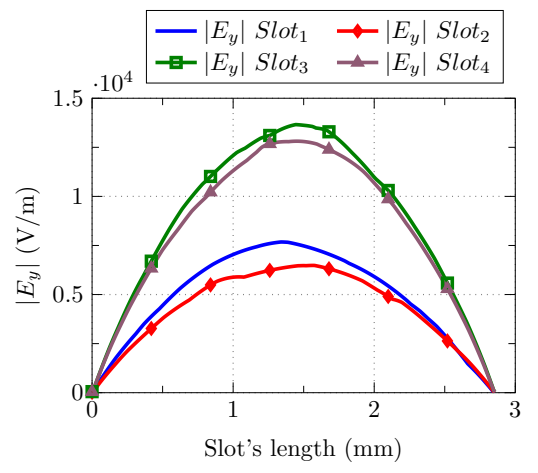

(a)

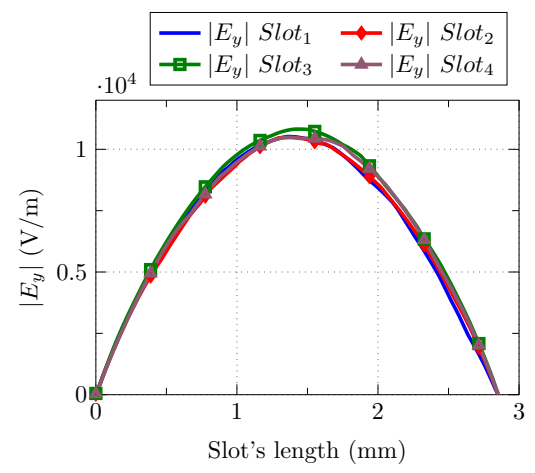

(b)

Figure 3.14: (a) Amplitude of the electric field in the apertures using a centered slit and (b) an off-center slit.
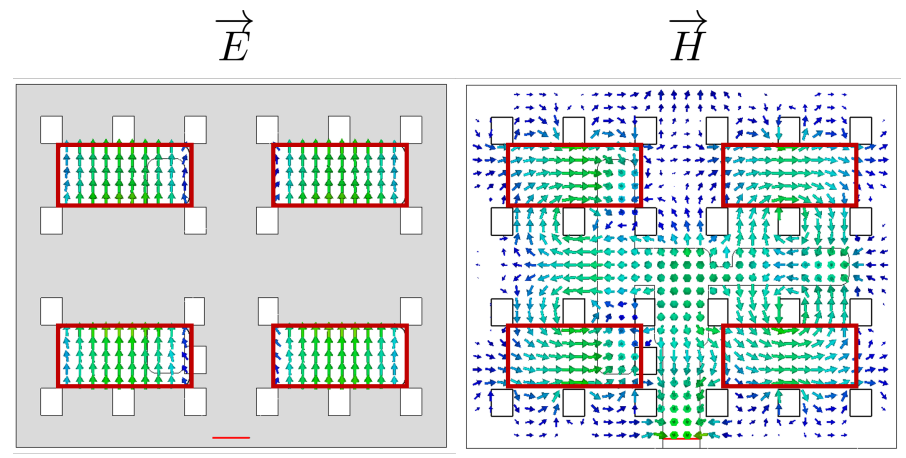

Figure 3.15: $\mathrm{E}$ and $\mathrm{H}$ field in a $2 \times 2$ cavity-backed slot antenna.

For this case, two possible slot arrangements are shown in Fig. 3.11, both leading to an array with unequally spaced apertures though. An effective solution to compensate the $180^{\circ}$ phase shift is to add a second splitter in the left arm, resulting in an asymmetrical disposition as illustrated in Fig. 3.12. Such asymmetry leads to a not uniform amplitude excitation of the radiating apertures.

Fig. 3.13 shows a schematic drawing of the new unit cell with the most representative elements. In order to attain an equal power distribution in the divider, an off-center slit is introduced. The tuning elements of the slit are its width, length, and position. It is observed that it has a small rounding on both sides because a milling radius of $0.2 \mathrm{~mm}$ has already been considered. 

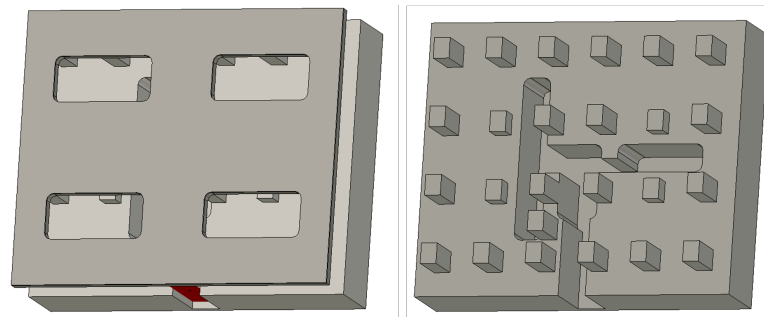

Figure 3.16: $2 \times 2$ cavity-backed slot-antenna fed by GGW.

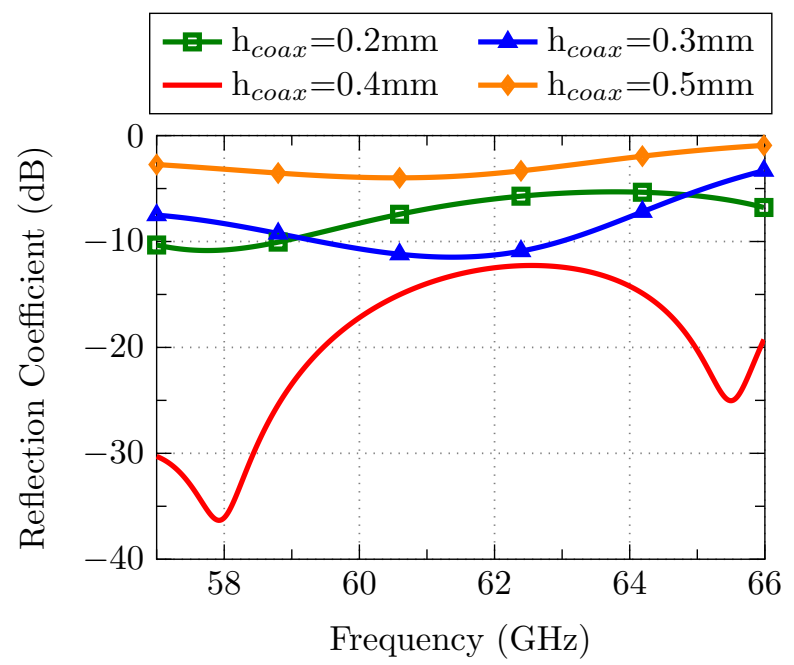

Figure 3.17: Reflection coefficient for different heights of the coaxial nails.

Placing the slit just in the center of the divider (off-center $=0$ in Fig. 3.13), the E-field magnitude on the apertures is different, as presented in Fig. 3.14a. However, if the slit is shifted to the right side, the E-field amplitudes can be compensated and finally equalized (Fig. 3.14b). The role of this slit is crucial to distribute evenly the power. Lastly, after fixing the position of the slit and having previously designed the optimal height of the coaxial nails, the same for all the cavities, a uniform field distribution can be obtained in the four apertures of the subarray, as shown in Fig. 3.15.

Finally, Fig. 3.16 shows the $2 \times 2$ subarray. The rectangular apertures are excited by coaxial cavities which are fed by the asymmetric GGW corporatefeed network. Fig. 3.17 shows a parametric study of the height of the coaxial nails to notice its relevant influence on the subarray performance. 


\subsubsection{Ridge Gap Waveguide feeding}

As pointed out at the beginning of the chapter, a ridge gap waveguide also manages to feed coaxial cavities. In a RGW, the field propagates between the ridge and the metallic top plate, more confinedly than in a horizontallypolarized GGW. However, by adjusting the distance between the short circuit of the RGW with respect to the cavity it is possible to achieve a good coupling. Following a similar scheme to the previous one, a $2 \times 2$ cell is taken as a demonstrator (Fig. 3.18).
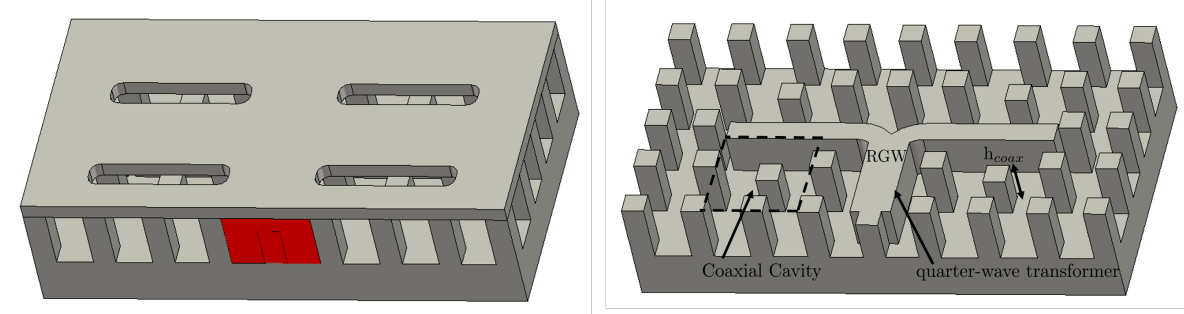

Figure 3.18: $2 \times 2$ Cavity-backed slot array fed by RGW.

The regular nails in this $\mathrm{V}$-band subarray are $1.25 \mathrm{~mm}$ high $\left(\lambda_{0} / 4\right.$ at $60 \mathrm{GHz}$. Regarding the coaxial nail, for the previous case fed by GGW, the height was set to $0.4 \mathrm{~mm}$ to achieve the cavity resonance. Conversely, for the RGW case, the coaxial nail must be longer since the field is more confined between the ridge and the top plate. In fact, the coaxial nail has to be as high as the ridge $(0.85 \mathrm{~mm})$ to achieve a proper coupling between them (Fig. 3.19).

Nonetheless, an important drawback of this approach is the way in which the apertures must be arranged. From Fig. 3.20 it is observed that there are two optimal arrangements to feed the apertures coherently. In both cases, a pair of apertures is necessarily closer to the RGW than the other pair. Such imbalance leads to an unequal distribution since the apertures closer to the RGW become more excited, as observed in Fig. 3.21. This fact does not have a noticeable impact at this subarray level, but it would be relevant in a larger array. Note that there would be consecutive rows of apertures more excited than others, thus degrading the radiation pattern performance.

This behavior is more easily observable in Fig. 3.20, where the magnitude of the magnetic field on the apertures is represented. To revert this effect, the nails of the coaxial cavities corresponding to the outermost apertures are moved outwardly with respect to the RGW. Fig. 3.22 shows this correction. By displacing the shortened nails (in purple) of the lower cavities, the magnitude of the electric field on the apertures becomes uniform. Despite this effective 
CHAPTER 3. RADIATING ELEMENTS FOR GAP

WAVEGUIDE ARRAYS

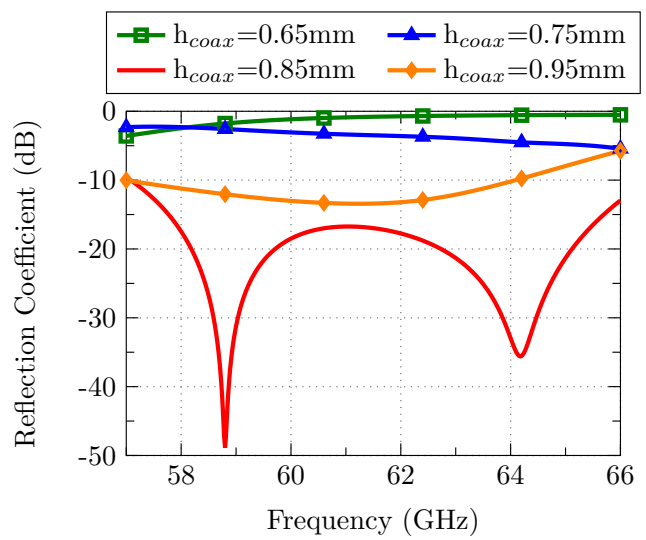

Figure 3.19: Reflection coefficient for different heights of the coaxial nail.

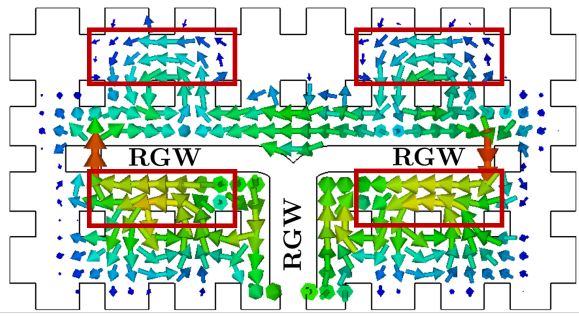

(a)

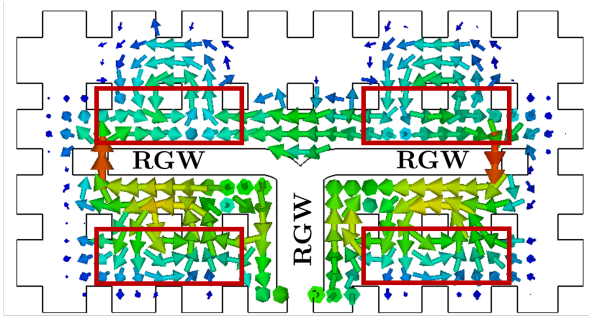

(b)

Figure 3.20: $\mathrm{H}$ field in a $2 \times 2$ cavity-backed antenna fed by RGW. The displayed field is sampled at the height of the shortened nail.

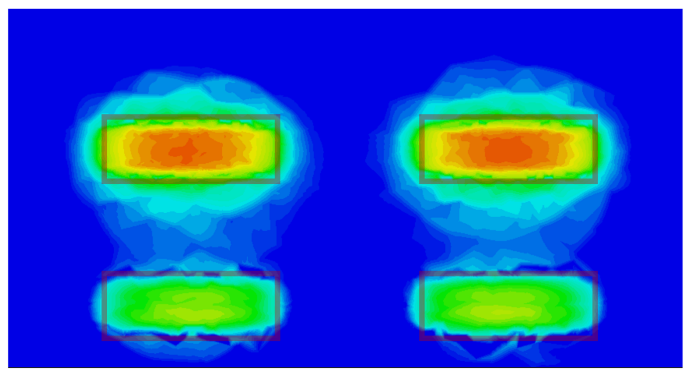

Figure 3.21: E field in a $2 \times 2$ cavity-backed antenna fed by RGW. The displayed field is sampled at the height of the apertures. 

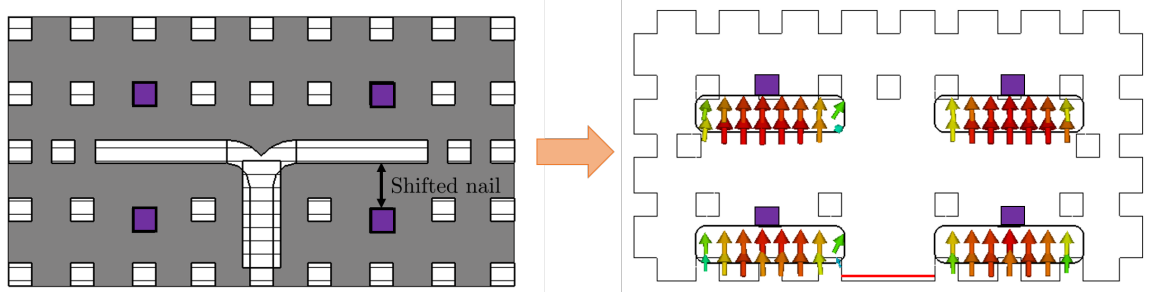

Figure 3.22: Field uniformization on the apertures by moving the nails of the coaxial cavities.

solution, the fact of needing more room to implement the RGW network reduces its attractiveness compared to the horizontally-polarized GGW feeding.

\subsubsection{GGW-RGW feeding}

As it has been developed throughout Chapter 2, an optimal solution has been conceived to uniformly excite arrays in a compact way. It consists of a hybrid network combining GGW and RGW. Following the same scheme of previous examples, $2 \times 2$ radiating apertures are fed by a GGW-RGW network. This subarray is shown in Fig. 3.23.
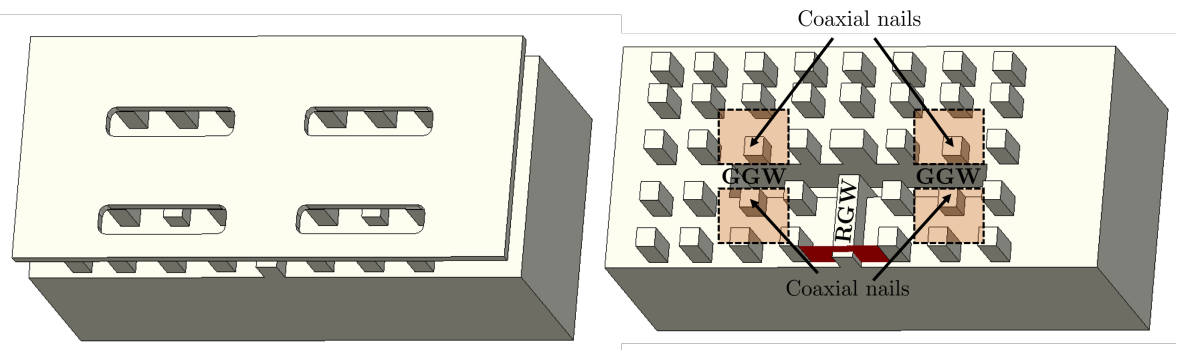

Figure 3.23: $2 \times 2$ cavity-backed slot array fed by GGW-RGW

In Fig. 3.24 the magnetic field for this sample is represented. Also in this same figure the location of the coaxial cavities is marked. In this case, the network does not need an extra arm on the left side to compensate for the phase difference. Besides, the network is vertically more compact compared to the RGW version. The simulated reflection coefficient using different coaxial heights is shown in Fig. 3.25, where a good matching performance is obtained for $h_{\text {coax }}=0.65 \mathrm{~mm}$. 


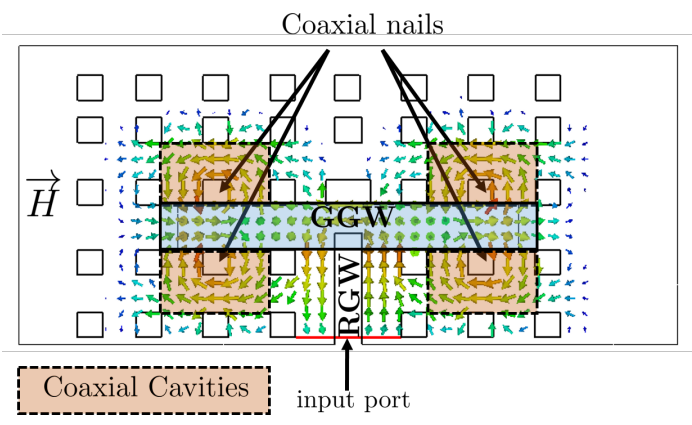

Figure 3.24: $\mathrm{H}$ field in the subarray fed by the RGW-GGW network. RGW, GGW and the position of the coaxial cavities are indicated.

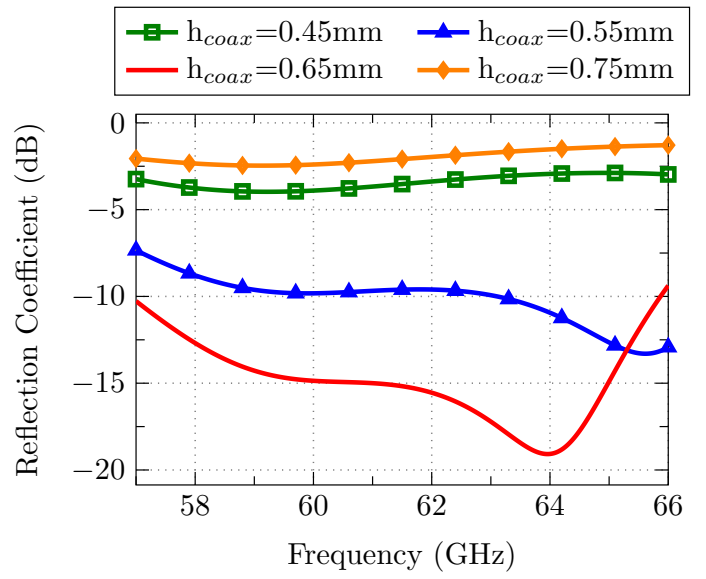

Figure 3.25: Reflection coefficient for different heights of the coaxial nail.

\subsubsection{Dual-Band Antennas}

We have seen how to host coaxial cavities and the feeding network in the same layer. Taking this solution as a starting point, one may try to extend this idea to host cavities with widely separated frequency bands within the same layer. Then, single-layer dual-band antennas could be conceived. Such solutions would be very attractive in SATCOM on the move applications, where $\mathrm{K}$-band is used to receive the signal and Ka-band to transmit it. Low-profile solutions typically use two dedicated antennas for this dual-band operation.

Bearing in mind that coaxial cavities must be around a quarter of the wavelength in height, it is possible to modify the resonance frequency by tuning the distance of the cavity floor with respect to the surrounding nails. Fig. 3.26 

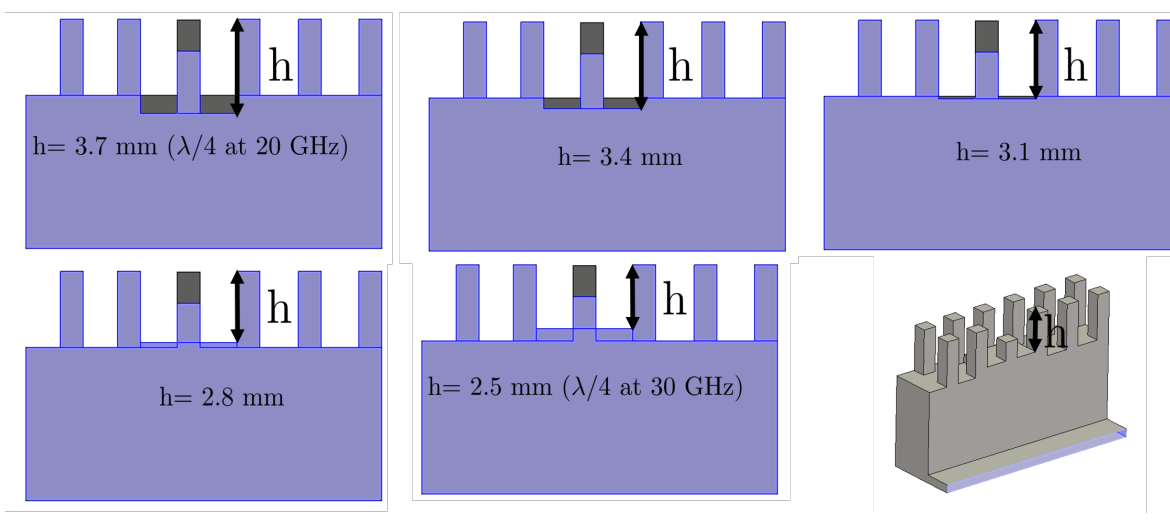

(a)

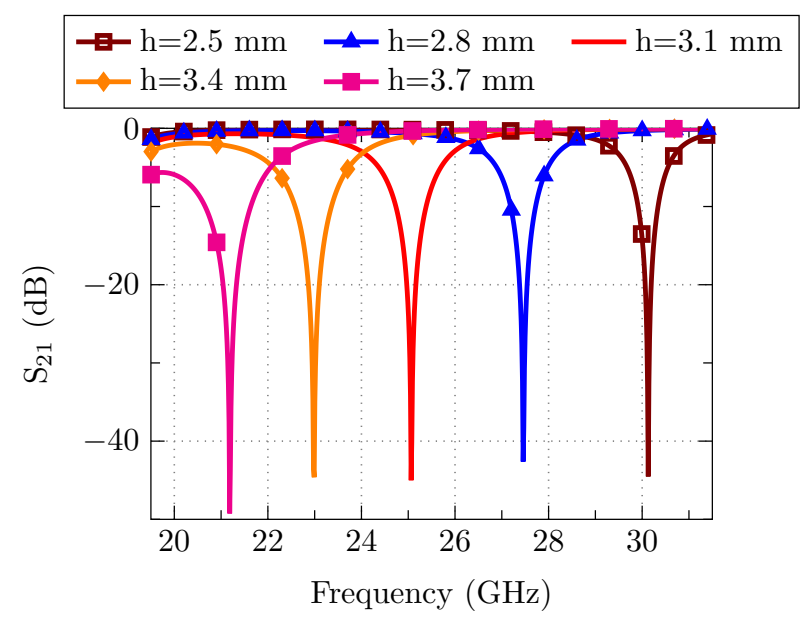

(b)

Figure 3.26: Transmission coefficients by varying the coaxial cavity height.

corroborates that, as the cavity ground moves away or approaches the lid, the resonance is shifted. A larger distance between the lid and the floor leads to a resonance at a lower frequency. On the other hand, if the floor is moved up, the resonance shifts to higher frequencies. Therefore, the resonance frequency of the coaxial cavities can be easily changed by tuning the height of the floor. This concept opens the door to host resonant cavities at widely separated frequencies in the same layer by simply adjusting the height of each cavity. 
Note that stopband bandwidth typically reaches an octave (Fig. 3.27), being enough to cover the two desired bands in $\mathrm{K}$ and Ka-band.

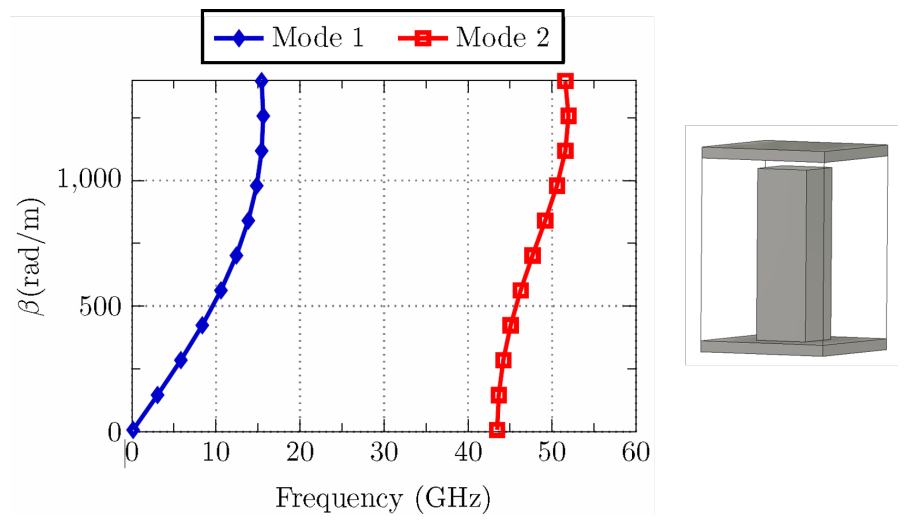

Figure 3.27: Stopband created for a nail height of $3.125 \mathrm{~mm}$, period of $2.25 \mathrm{~mm}$ and width of $1 \mathrm{~mm}$.

Upper-band Cavity

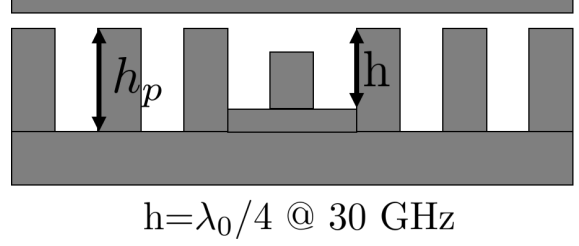

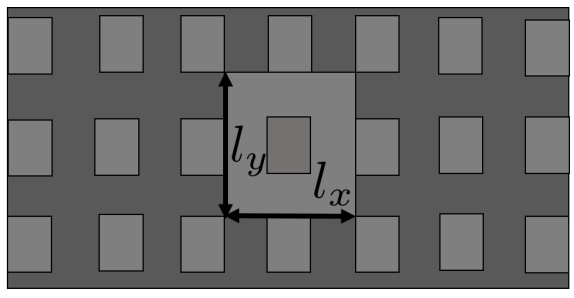

Figure 3.28: Side and top view of the cavity at $30 \mathrm{GHz}$.

\section{Upper Band Cavity}

Since the ultimate goal is to design a dual-band low-profile antenna for SATCOM applications, cavities resonating at $20 \mathrm{GHz}$ and $30 \mathrm{GHz}$ has been pursued. Fig. 3.28 shows a cavity where the floor has moved upwards $(h=2.5 \mathrm{~mm}$ and $\left.h_{p}=3.125 \mathrm{~mm}\right)$. As a consequence, the cavity resonance is shifted to a higher frequency, in this case $30 \mathrm{GHz}$.

One interesting feature is that the periodicity and width of the nail do not have to be altered. The two only tuning parameters are the height of the cavity floor $(h)$, as a coarse tuning, and the coaxial height $\left(h_{\text {coax }}\right)$ as a fine tuning. A side and top view of the cavity is shown in Fig. 3.28. The values of $l_{x}$ and $l_{y}$ are determined by the thickness of the nails and the period between them. 
In this regard, the choice of the nail periodicity will be crucial to place the cavities as close as possible. In Fig. 3.29 a perspective view of the cavity and the excited magnetic field loop at $30 \mathrm{GHz}$ are shown. In Fig. 3.30 the response of the S-parameters is observed.
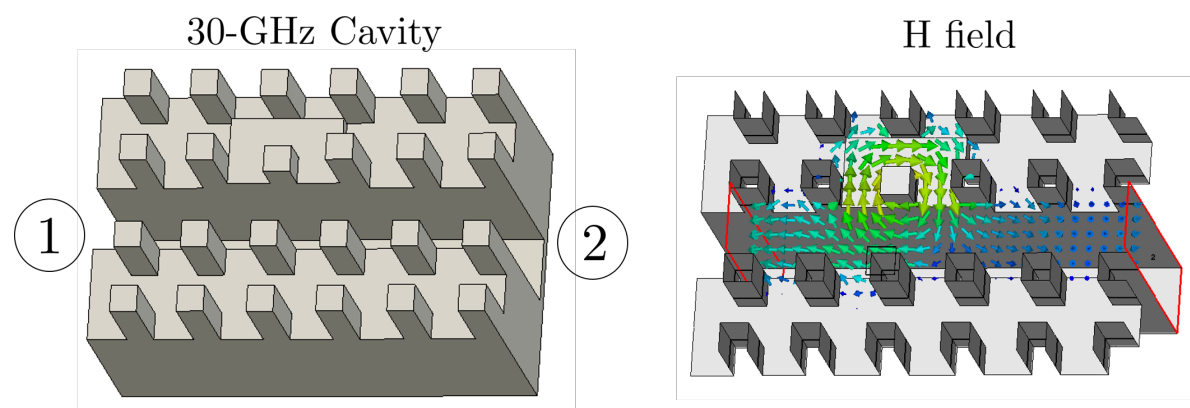

Figure 3.29: (a) Cavity operating at $30 \mathrm{GHz}$ and (b) magnetic field distribution.

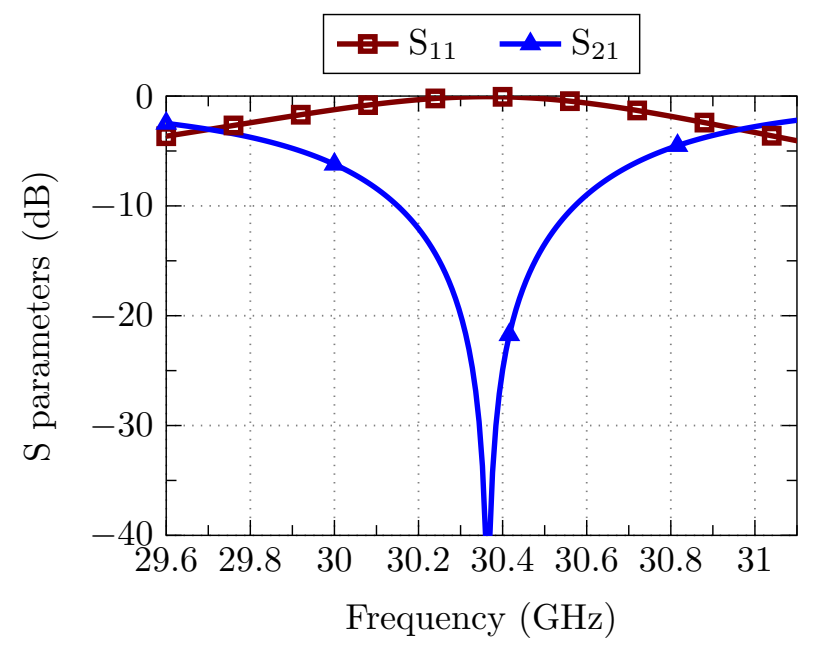

Figure 3.30: S-parameters of the $30-\mathrm{GHz}$ cavity.

\section{Lower Band Cavity}

For the cavity resonating at $20 \mathrm{GHz}$ the floor is moved down. Now $h$ is $3.75 \mathrm{~mm}$, which corresponds to $\lambda_{0} / 4$ at $20 \mathrm{GHz}$ (Fig. 3.31). The height of the nails $h_{p}$ is $3.125 \mathrm{~mm}$, as in the upper band cavity. This way, the excavation depth in 
CHAPTER 3. RADIATING ELEMENTS FOR GAP WAVEGUIDE ARRAYS

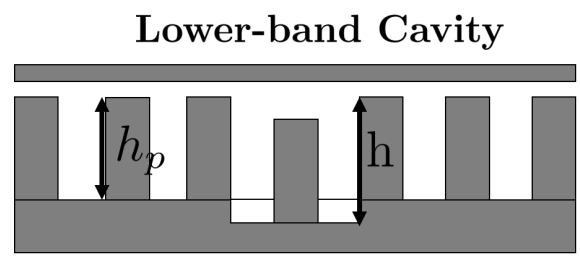

$$
\mathrm{h}=\lambda_{0} / 4 @ 20 \mathrm{GHz}
$$

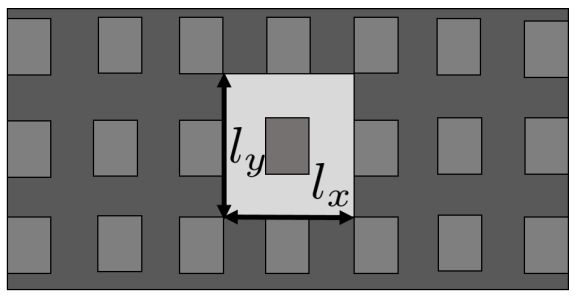

Figure 3.31: Side and top view of the cavity at $20 \mathrm{GHz}$.
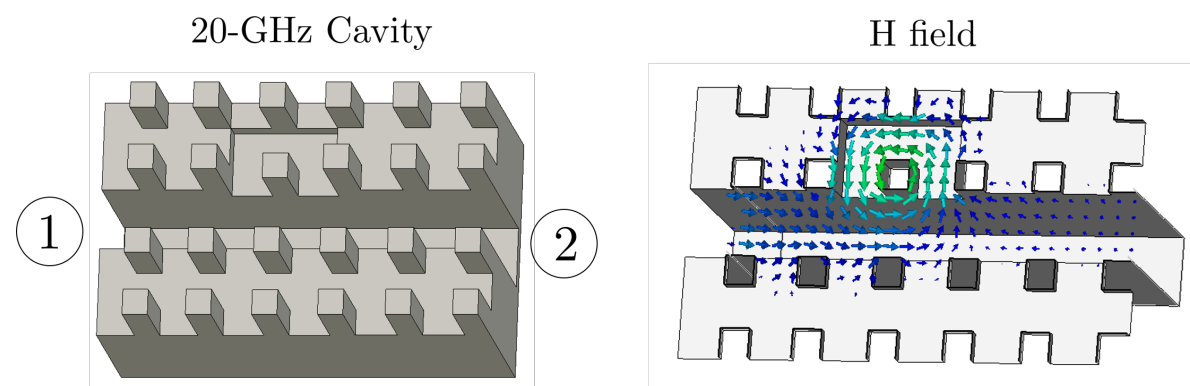

Figure 3.32: (a) Cavity operating at $20 \mathrm{GHz}$ and (b) magnetic field distribution.

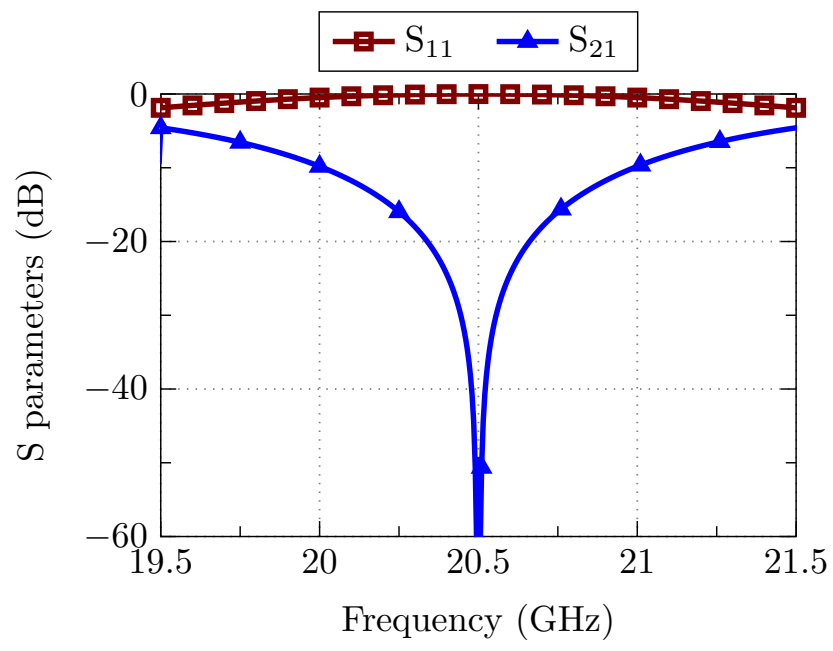

Figure 3.33: S-parameters of the 20-GHz cavity. 
the lower band cavity and the mound height in upper band cavity is the same, $0.625 \mathrm{~mm}$.

Fig. 3.32 shows the perspective view of the cavity and the magnetic field loop generated at $20 \mathrm{GHz}$. Now, since the floor of the cavity is moved down, the frequency has shifted to a lower frequency and a very strong resonance is observed at $20.5 \mathrm{GHz}$ (Fig. 3.33).

\section{$2 \times 2$ Dual-Band Slot Array}

Once the feasibility of housing resonant cavities at two widely separated bands within the same bed of nails has been demonstrated, a $2 \times 2$ subarray with 2 cavities of each type is presented. In this first example, half of the cavities have been designed at $20 \mathrm{GHz}$ and the other half at $30 \mathrm{GHz}$.

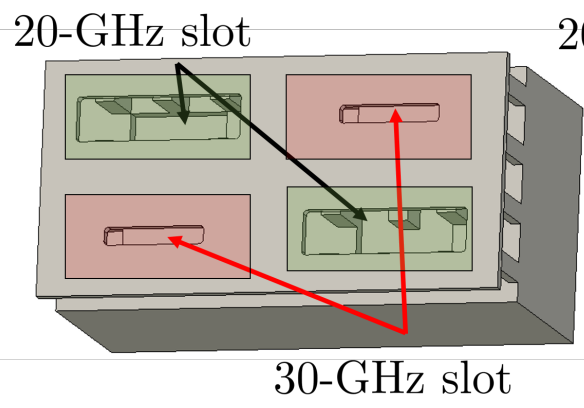

20-GHz cavity

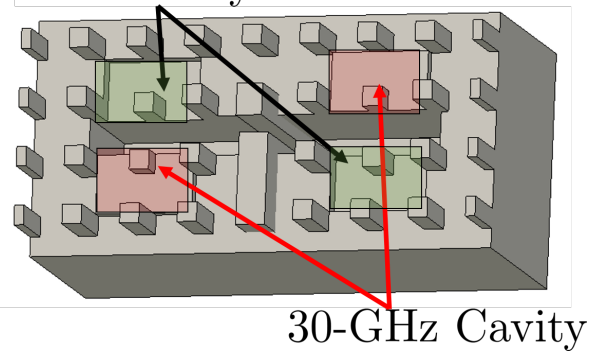

Figure 3.34: $2 \times 2$ Dual-band cavity-backed slot-antenna fed by a combined RGW-GGW network.

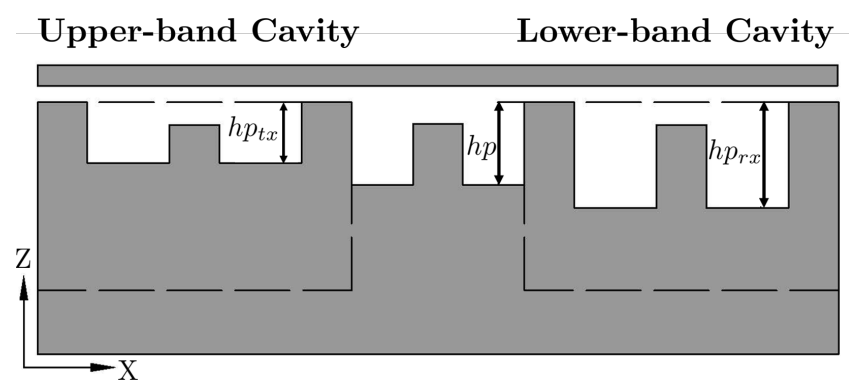

Figure 3.35: Side view of a bed of nails housing cavities of different heights.

One of the key aspects to design a dual-band array in a single layer is the disposition of the radiating apertures as well as the cavities excitation. It is not an easy challenge since apertures are excited in very separated bands. All the 


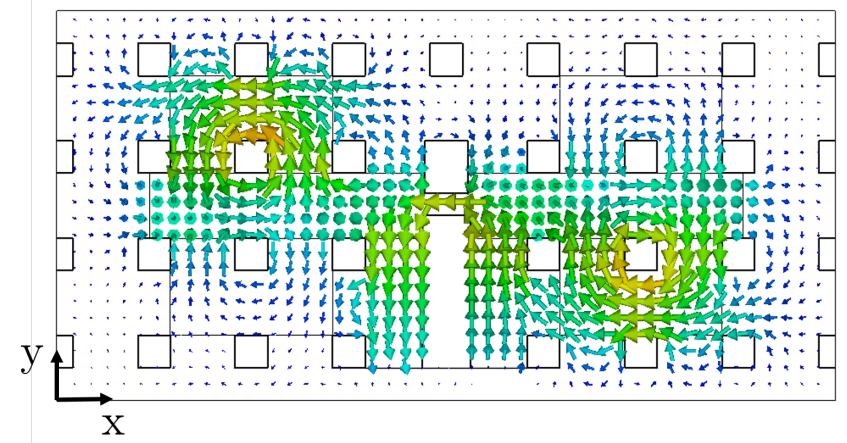

(a)

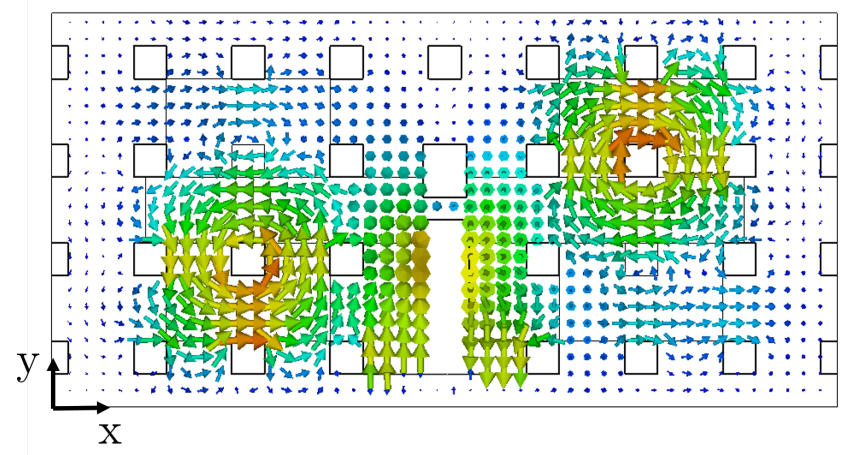

(b)

Figure 3.36: (a) H-field at $20 \mathrm{GHz}$ and (b) H-field at $30 \mathrm{GHz}$.

details will be given in Chapter 4, but an arrangement alternating RX and TX apertures is adopted, shown in Figs. 3.34 and 3.35. The spacing between the apertures is $12 \mathrm{~mm}$ in the X-plane and $6 \mathrm{~mm}$ in the Y-plane. Therefore, since $\lambda_{0}$ is $10 \mathrm{~mm}$ at $30 \mathrm{GHz}$, TX apertures are spaced more than one wavelength in the X-plane. Consequently, grating lobes will appear along the XZ plane in the upper band. Fig. 3.36 shows how the H-field is trapped around the coaxial nails. As expected, upper band cavities are excited at $30 \mathrm{GHz}$ and the lower band cavities at $20 \mathrm{GHz}$. Finally, Fig. 3.37 shows the simulated radiation patterns for both main planes. A $4 \times 4$ array factor has been applied to the basic cell to emulate a larger array.

In short, the coaxial cavities implemented in GW technology revealed themselves as an effective solution to implement simpler approaches of dual-band arrays. The versatility of these cavities allows to achieve, in a single layer, 


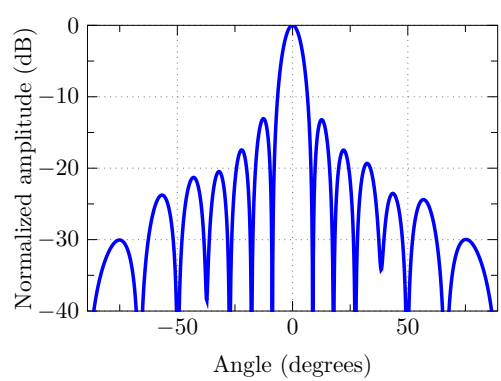

(a)

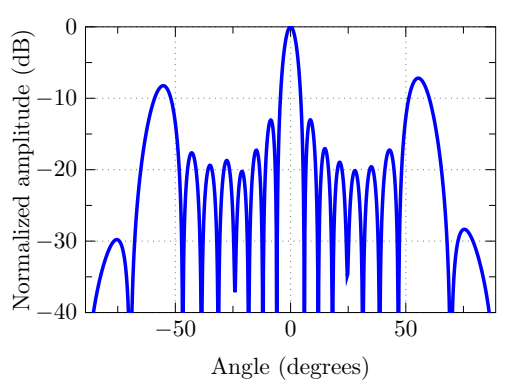

(c)

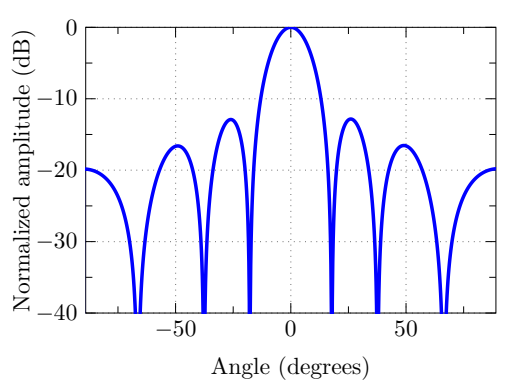

(b)

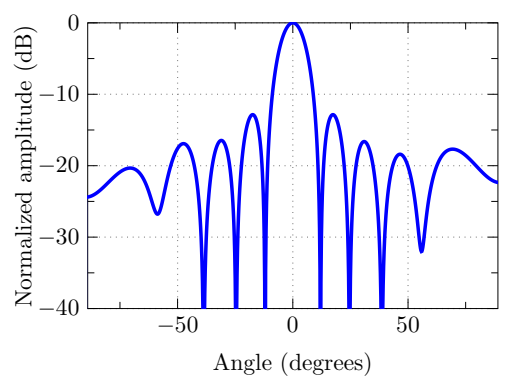

(d)

Figure 3.37: Radiation patterns at both bands: (a) XZ-plane (20 GHz); (b) YZ-plane (20 GHz); (c) XZ-plane $(30 \mathrm{GHz})$; (d) YZ-plane $(30 \mathrm{GHz})$.

resonant cavities at different frequencies just by adjusting their vertical size. This fact enables practical designs of high-gain antennas able to share in the same panel two arrays working in widely separated frequency bands.

\subsection{Cylindrical Cavities}

In this section, alternative radiating elements based on cylindrical cavities are presented. In the strict sense of the word, though, these cavities are not resonant but rather a guided medium through which the signal propagates and couples to radiating circular apertures. The term cylindrical cavity will be used hereinafter. Fig. 3.38 shows the basic model of the radiating element. It is a circular waveguide fed at one of its sides. Obviously, the cylinder could also be fed from the back. In that case, it would basically behave as an open circular waveguide. The employed lateral feeding is more convenient for a single-layer architecture making use of a corporate feeding network. 

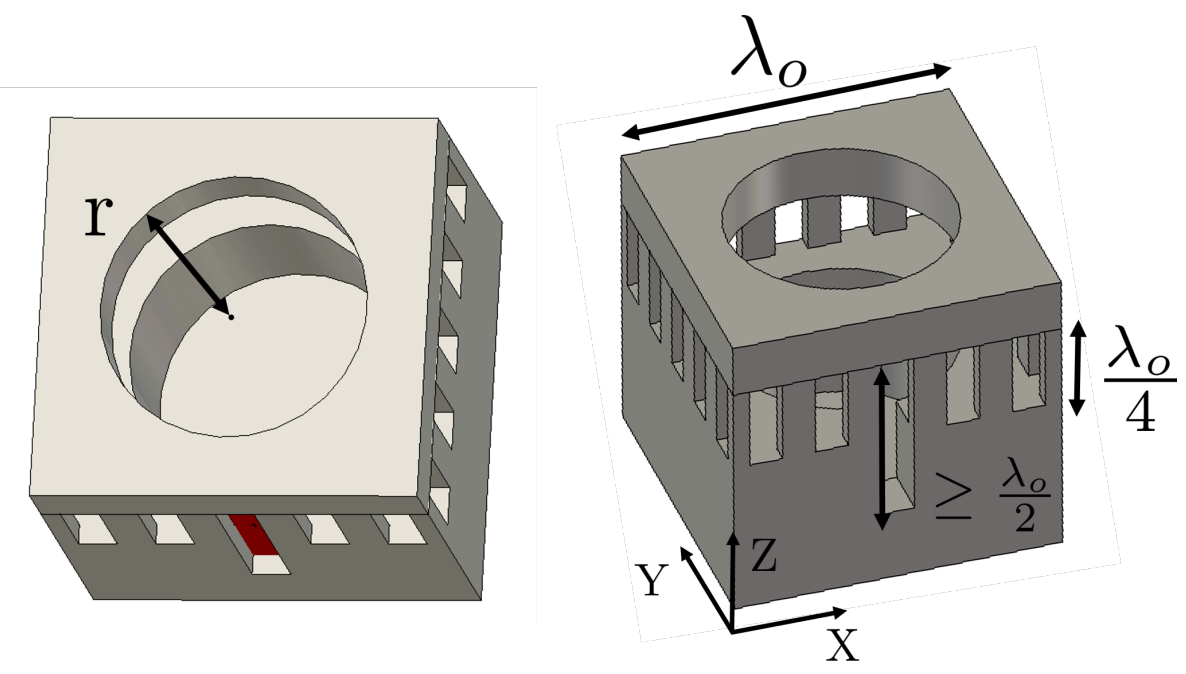

Figure 3.38: Different views of the cylindrical cavity.

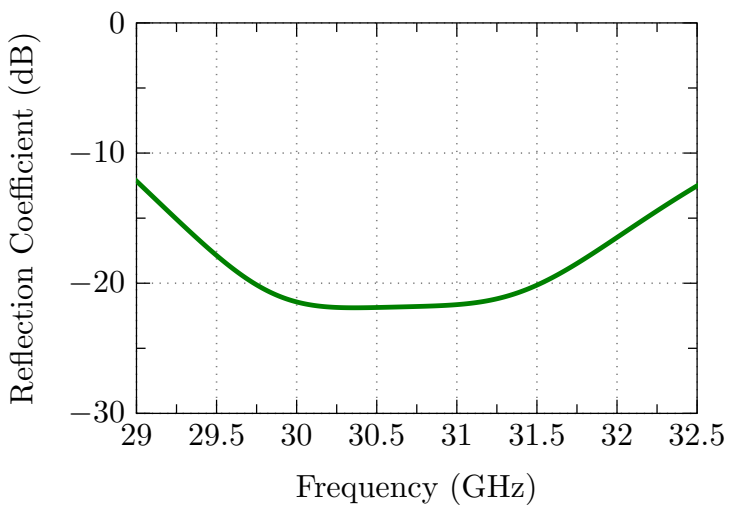

Figure 3.39: Reflection coefficient of the basic cell using a cylindrical cavity fed by a GGW.

As it will be discussed later, this feeding mechanism enables to conceive new array concepts, such as dual-polarized, circularly-polarized or dual-band antennas. In the coaxial cavity case, it would be difficult to get dual-polarized antennas in a single layer. A possible solution would be to add a second layer. Thus, each layer could radiate with orthogonal linear polarizations. Anyway, stacking coaxial cavities does not seem evident with the proposed architecture. 


\subsection{Cylindrical Cavities}

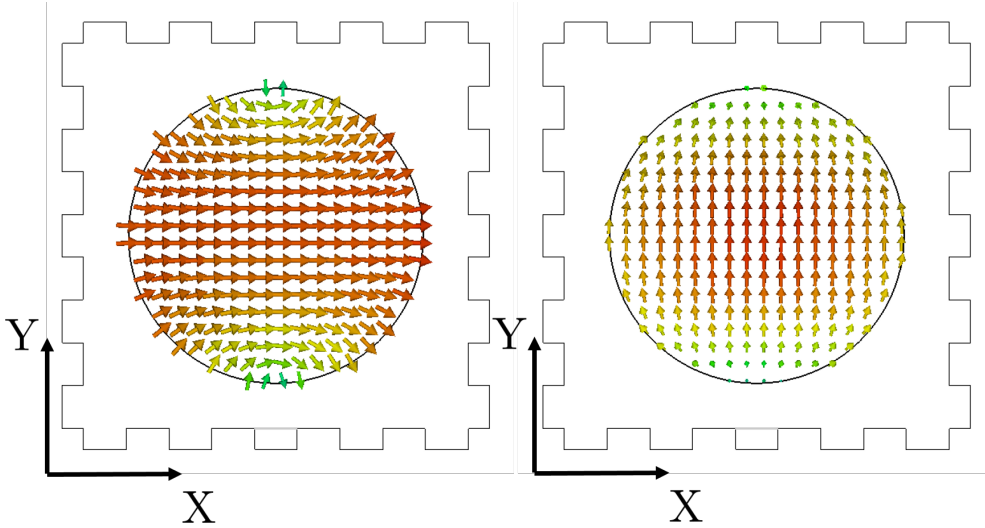

Figure 3.40: (Left) $\mathrm{E}_{x}$ and (right) $\mathrm{H}_{y}$ on a radiating element based on a cylindrical cavity.
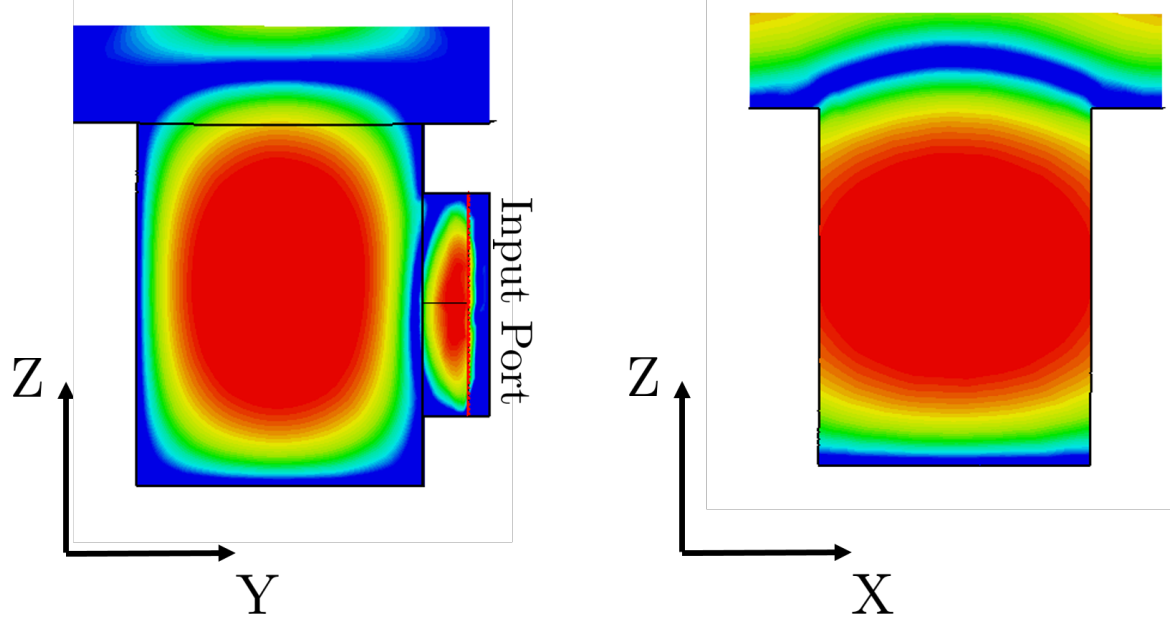

Figure 3.41: $E_{x}$ field component in $\mathrm{YZ}$ and $\mathrm{XZ}$ planes for the radiating element based on a cylindrical cavity.

With cylindrical cavities, however, it seems simpler to excite them at different heights to achieve dual-polarized radiators.

When using the cylindrical cavities as radiating elements, the main tuning parameter is the radius of the aperture, which is approximately determined by $\mathrm{r} \simeq \lambda_{0} / \pi$, i.e., a diameter of $0.64 \lambda_{0}$. The rest of tuning parameters are 


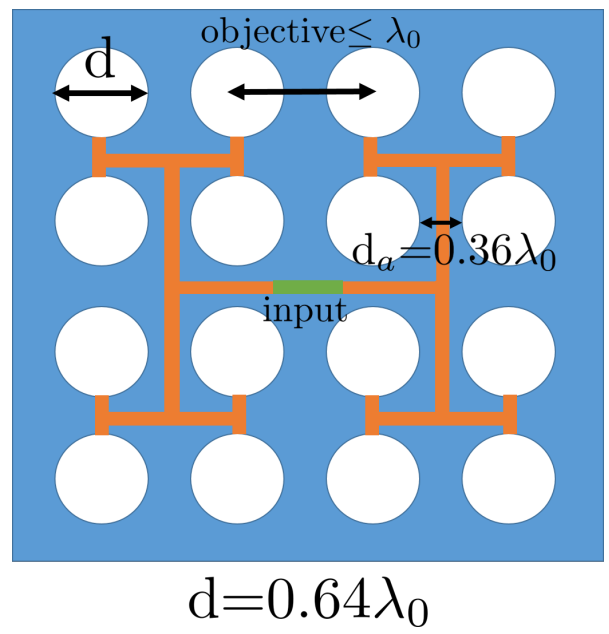

Figure 3.42: Outline of a $4 \times 4$ array using cylindrical cavities. The room left to locate the corporate distribution network is less than $0.36 \lambda_{0}$ in both dimensions.

indicated in Fig. 3.38. For a unit cell working at $30 \mathrm{GHz}$, the nails height and the aperture radius is $2.5 \mathrm{~mm}$ and $3.2 \mathrm{~mm}\left(\lambda_{0} / \pi\right)$ respectively. The simulated $S_{11}$ is plotted in Fig. 3.39, achieving a bandwidth of $13 \%$ for $S_{11}<-10 \mathrm{~dB}$.

Regarding the field on the aperture, a uniform electric field is observed in Fig. 3.40. In this figure, the distribution of electric and magnetic fields on the circular aperture can be observed. A great polarization purity is achieved. In Fig. 3.41 the amplitude of the $E_{x}$ component is represented in two cuts corresponding to the $\mathrm{YZ}$ and $\mathrm{XZ}$ planes. The colored graphs show how the electric field is coupled from the input port to the radiating circular aperture.

Keeping in mind the final goal of designing two-dimensional arrays, the schematic situation can be seen in Fig. 3.42 when cylindrical cavities are used. On the one hand, apertures should be located at a distance less than $\lambda_{0}$ both vertically and horizontally to avoid grating lobes. On the other hand, the diameter of the apertures must be around $0.64 \lambda_{0}$. So, the network must fit between the remaining $0.36 \lambda_{0}$ distance between the apertures. Verticallypolarized GGW network is discarded because it would be too wide (at least $\left.0.5 \lambda_{0}\right)$. Thus, the RGW or the horizontally-polarized GGW arise as possible options.

\subsubsection{Cylindrical Cavities fed by GGW}

The most immediate way to feed this type of cavity is through a horizontallypolarized GGW. This type of feeding has already been shown in Fig. 3.38. As 
can be observed, a feeding GGW fits in the room left between two adjacent nail rows. As it is well known, the cut-off frequency of a horizontally-polarized GGW is determined by its height. Therefore, narrow waveguides can be used to design compact networks fulfilling the space requirements shown in Fig 3.42. Let us now see the dimensions and performance of these cavities in a sample $2 \times 2$ array (Fig. 3.43).
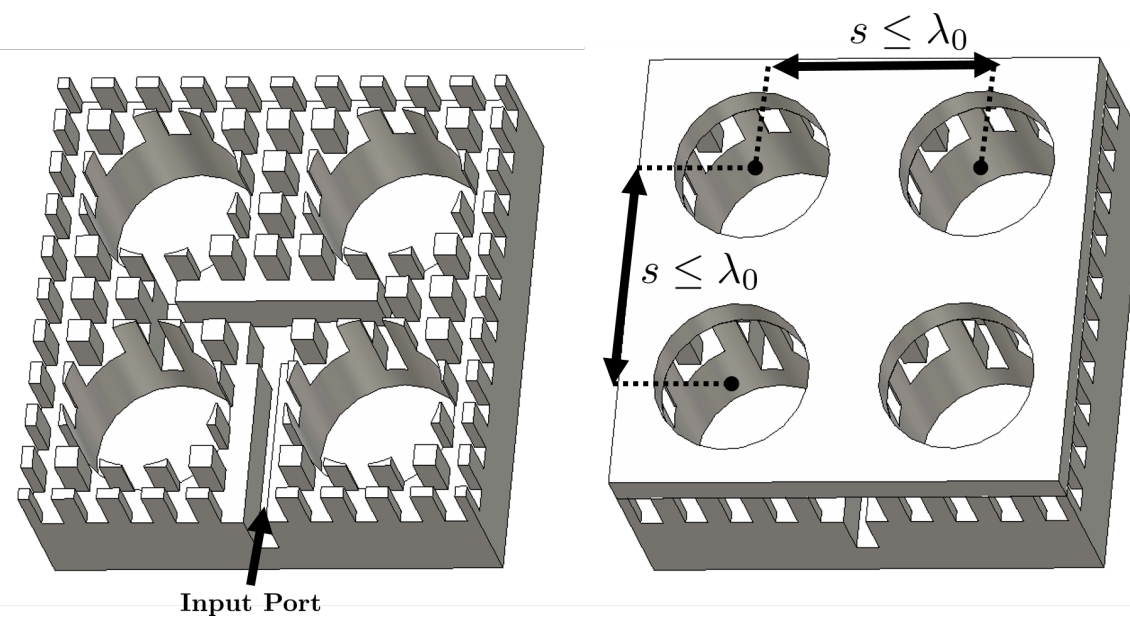

Figure 3.43: $2 \times 2$ Cylindrical cavities fed by GGW.

A horizontally-polarized GGW feeding network is implemented to distribute the signal between the unit cells, choosing a groove width of $1 \mathrm{~mm}\left(0.1 \lambda_{0}\right)$. However, as it was demonstrated throughout Chapter 2, the E-plane powerdividers produce a phase difference of $180^{\circ}$ in each of the arms. Therefore, if the distribution network only uses E-plane GGW dividers as building blocks, the cavities would be excited with opposite phases (Fig. 3.44), leading to a broadside null in the radiation patterns.

Different solutions could be implemented to solve this issue. As it was discussed already, the phase inversion in the power-dividers can be avoided using ridge waveguides. Then, the feasibility of coupling the RGW guided mode to the cylindrical cavity must be first demonstrated.

\subsubsection{Cylindrical Cavities fed by RGW}

The cylindrical cavity fed laterally through a RGW is shown in Fig. 3.45. The protrusion of the RGW inside the cavity ensures a proper field coupling. The reflection coefficient of the unit cell is shown in Fig 3.46, where a matching value better than $-10 \mathrm{~dB}$ is obtained in a $10 \%$ frequency bandwidth. 


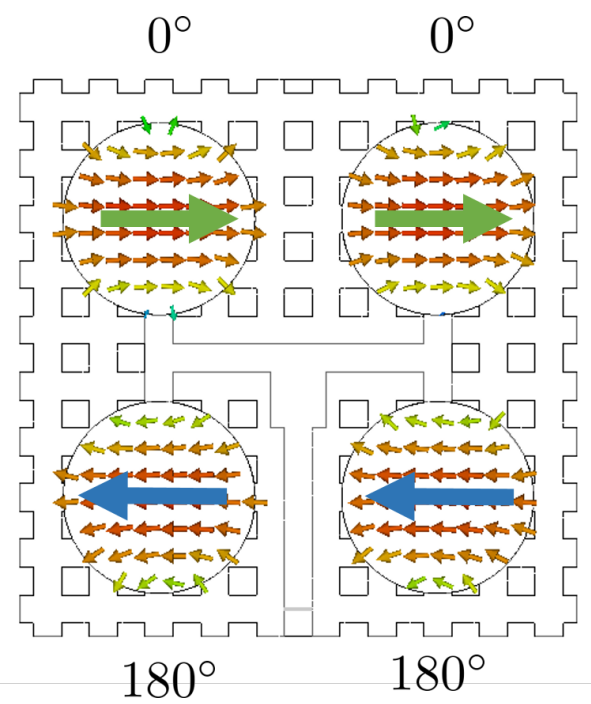

Figure 3.44: E-field in the circular apertures of a $2 \times 2$ antenna fed by GGW.
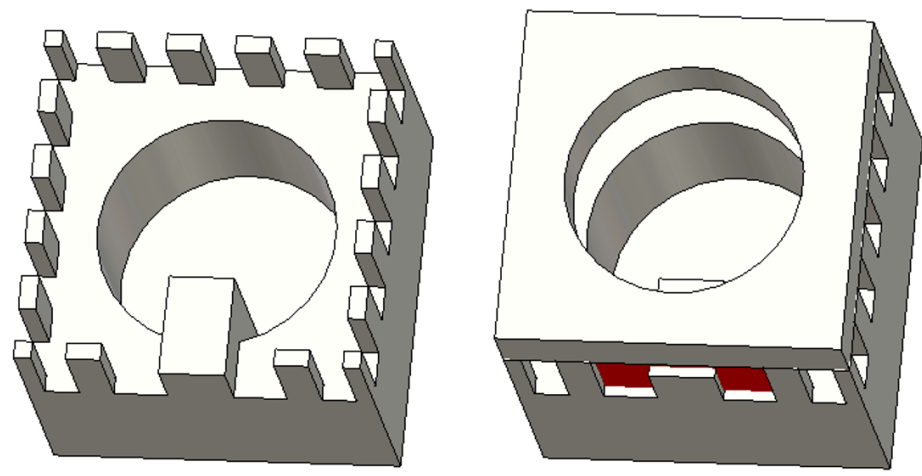

Figure 3.45: Cylindrical cavity fed by RGW.

Note that the RGW protrusion has not been fully optimized and even better results can be achieved, being this example a proof of concept. Therefore, a single radiator can be excited through an RGW feeding port. Extension to a two-dimensional array is more troublesome. The reason why the RGW alone does not allow compact designs has already been discussed. Fig. 3.47 shows the lack of room to place the RGW with enough isolation (orange box). 


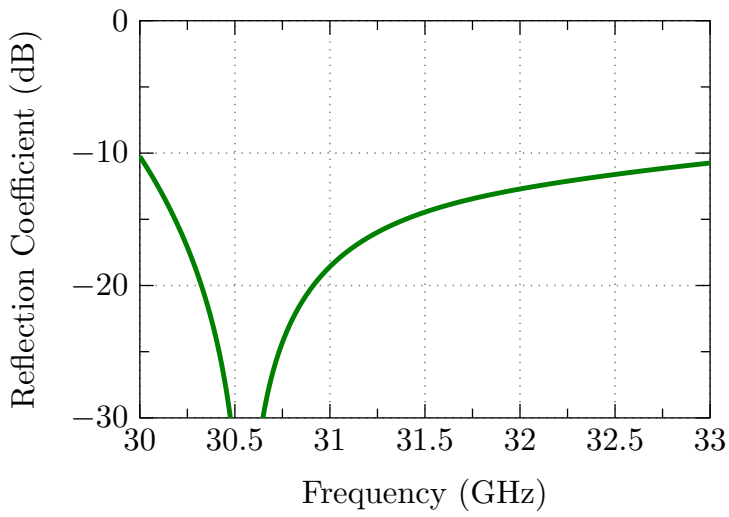

Figure 3.46: Reflection coefficient of the basic cell using a cylindrical cavity fed by a RGW.

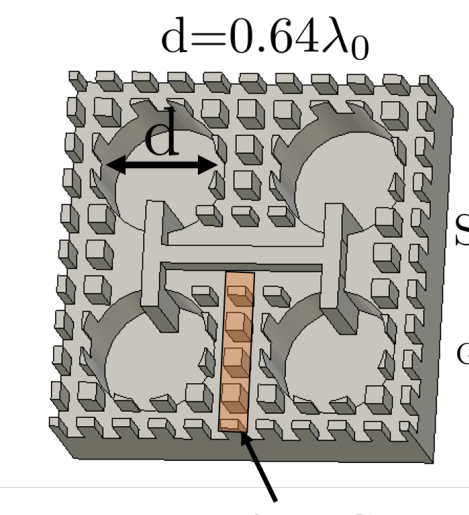

No space for RGW
Ridge Gap Waveguide

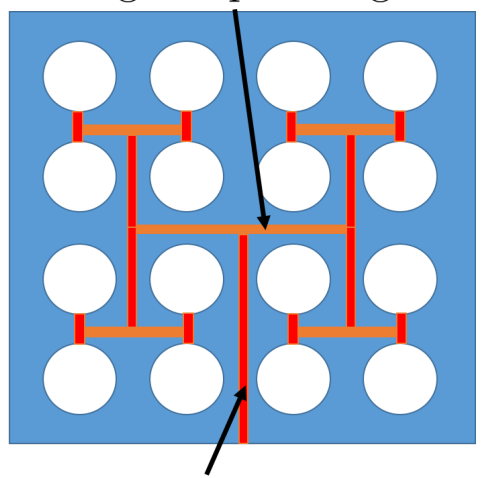

H-Pol Groove Gap Waveguide

Figure 3.47: Compact and symmetrical distribution network solution: a combined of RGW with GGW.

Consequently, the objective marked by Fig. 3.42 cannot be met by using RGW-based feeding networks. Notice that cavities and radiators might be separated to have enough room to place the RGW distribution network, but this action would lead to grating lobes on the radiation patterns. At this point, the effective solution reached for the coaxial cavities can also serve in this case. The hybrid RGW-GGW network can be compact enough to meet the discussed requirements, as sketched in Fig. 3.47. 


\subsubsection{Chamfered Cylindrical Cavities}

Taking the cylindrical cavities as starting point, it should be easy to modify them to achieve circular polarization. Keep in mind that this feature is one of the typical antenna requirements for SATCOM. The new radiating element is shown in Fig. 3.48, being a chamfered version of the previous circular aperture.

To achieve good circular polarization purity, two tuning parameters are used: the angle and the size of the chamfer. The shorter the cavity is, the more abrupt the chamfer must be to obtain two orthogonal modes excited with $90^{\circ}$ phase difference. Here the cavity is chosen high enough so that the chamfers are not too abrupt. This favors the access to the cavity from its side without difficulties by a groove gap waveguide.
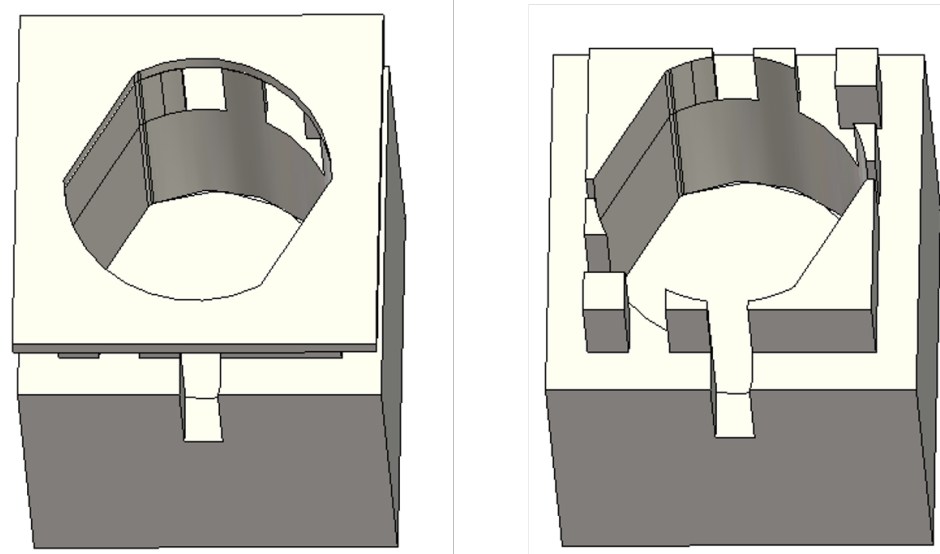

Figure 3.48: Circularly-polarized basic cell.

The fundamental aspect for this low-profile approach is the fact that the polarizer is embedded into the radiating cavity itself. This type of structure reminds a typical circularly-polarized patch design in microstrip technology (Fig. 3.49). A top view of the unit cell is shown in Fig. 3.50. The chamfers are not made of square-shaped nails but thick metal triangles to ensure the robustness of the piece. Note that this piece is essential to achieve a good circular polarization purity.

The unit cell can be extended to obtain a high-gain array by making use of a hybrid GGW-RGW corporate-feed network. A $2 \times 2$ sample array is shown in Fig. 3.51. An axial ratio below $3 \mathrm{~dB}$ is obtained from $29.5 \mathrm{GHz}$ to $31 \mathrm{GHz}$. It is a compact circularly-polarized subarray where the feeding network, the radiating cavities and the polarizer are hosted in the same layer. 


\subsection{Cylindrical Cavities}
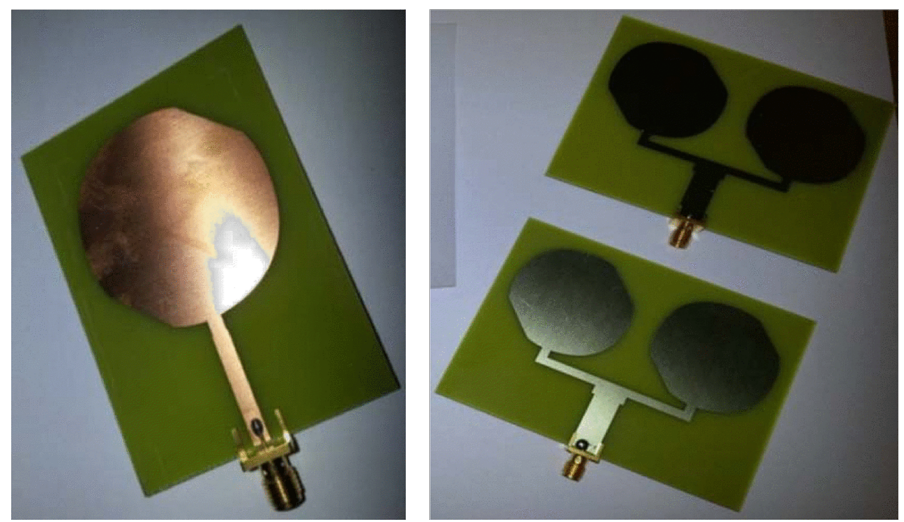

Figure 3.49: Fabricated dual patch antenna [228].

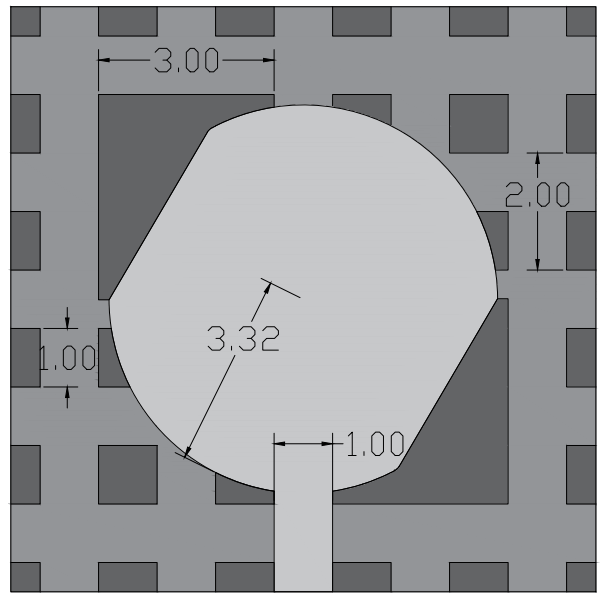

Figure 3.50: Top view and relevant dimensions of the basic cell.

\subsubsection{Dual-Polarized Cavities}

Antennas with dual polarization have also been explored. In this case, it is no longer a single-layer antenna but a multilayer one to achieve the dual operation. The lower layer radiates with linear polarization and the upper layer with the orthogonal component. The coupling between the feeding layer 1 and the feeding layer 2 is via a longitudinal slot. The radiating layer is shared by both polarizations.

The full structure is shown in Fig. 3.52. The cylindrical cavity is fed at different heights. In order to improve the isolation between both ports, a 


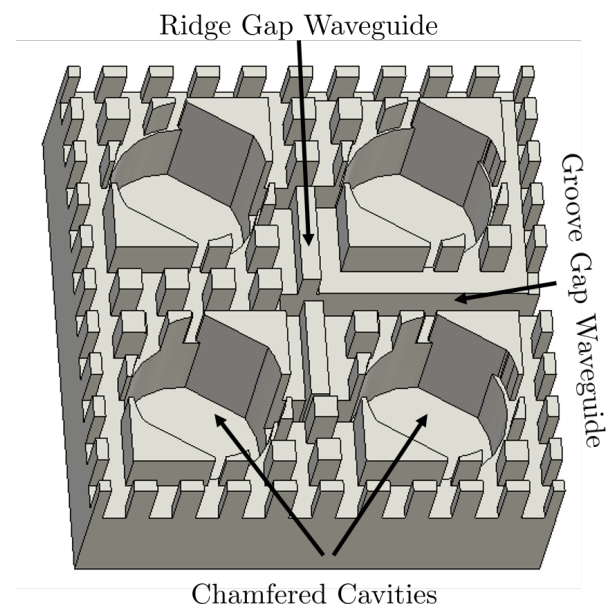

(a)

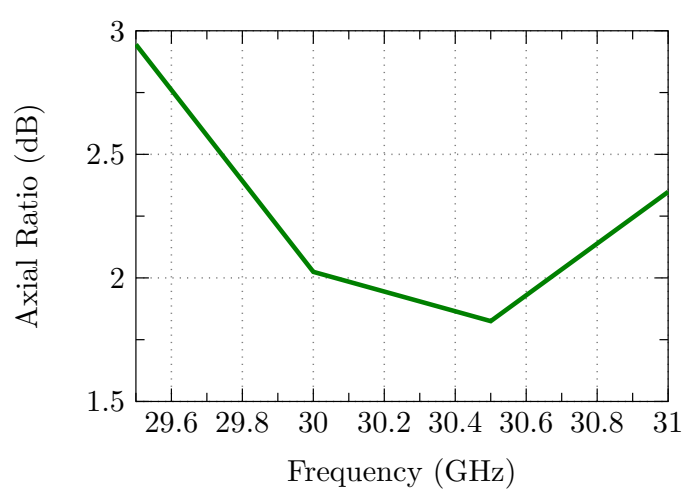

(b)

Figure 3.51: (a) $2 \times 2$ single-layer circularly-polarized antenna. (b) Simulated axial ratio.

longitudinal slot is placed in the middle of the cylinder. In Fig. 3.53 the different layers of the subarray are shown in a top view.

Fig. 3.54 shows the S-parameters of the $2 \times 2$ array cell. The reflection coefficients are not identical since the conditions are not the same for both levels. The lower level couples to the upper one by a longitudinal slot while the intermediate layer radiates directly through the circular aperture. Nonetheless, both reflection coefficients are below $-10 \mathrm{~dB}$ from $29.5 \mathrm{GHz}$ to $31 \mathrm{GHz}$. The isolation between ports is greater than $40 \mathrm{~dB}$ within the whole bandwidth. 


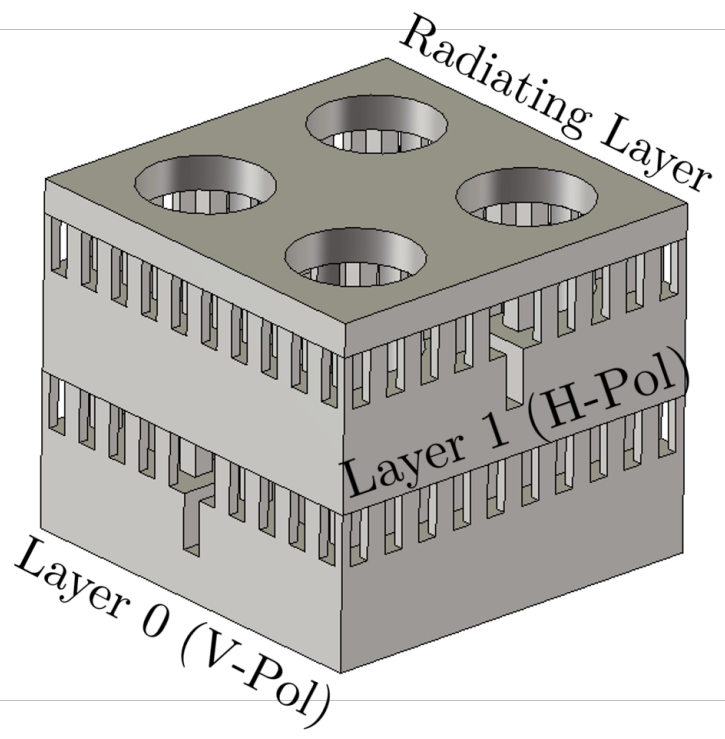

Figure 3.52: $2 \times 2$ dual-polarized antenna.

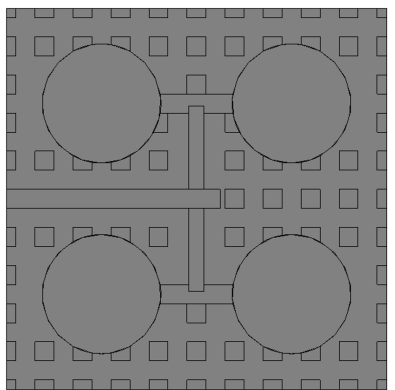

Layer 0 (V-Pol)

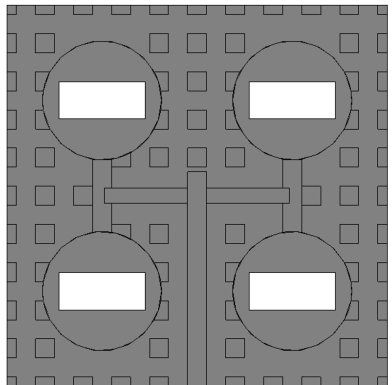

Layer 1 (H-Pol)

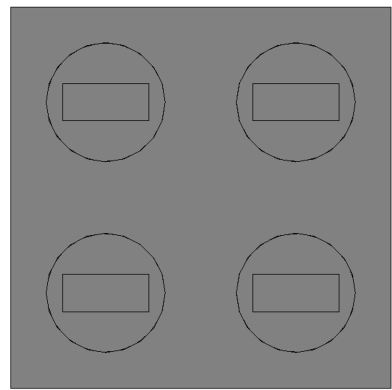

Radiating Layer

Figure 3.53: Layers of the dual-polarized antenna from bottom to top.

\subsubsection{Dual-Band Cavities}

The previous scheme explored the possibility to feed arrays through different layers to achieve a dual operation. Following the same concept, dual-band antennas in GW are also feasible.

As seen at the beginning of this section, the diameter of the circular cavity determines its working frequency. Keeping this in mind, two stacked cavities with different diameters can be combined for a dual frequency operation. The 


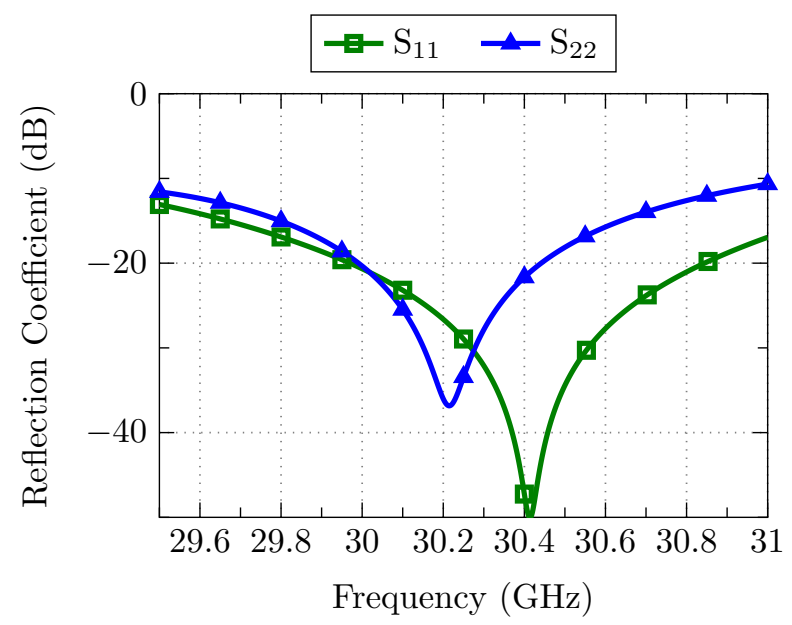

(a)

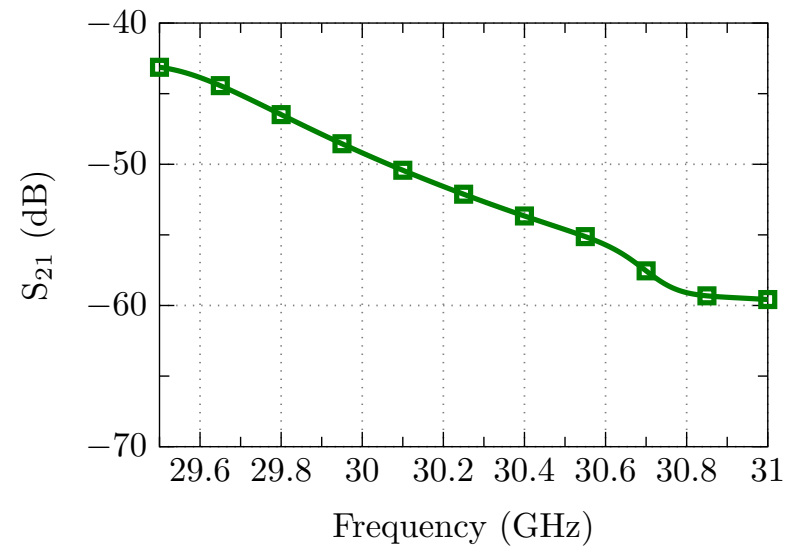

(b)

Figure 3.54: S-parameters of the $2 \times 2$ dual-polarized antenna.

targeted bands are the same used for the single-layer solution based on coaxial cavities. Hence, the diameter of the aperture working at $20 \mathrm{GHz}$ is $\mathrm{d}=9.5 \mathrm{~mm}$ while that operating at $30 \mathrm{GHz}$ is $\mathrm{d}=6.5 \mathrm{~mm}$. Obviously, the $30-\mathrm{GHz}$ cavity is placed below, so the fields at $30 \mathrm{GHz}$ can propagate through the cylindrical 20$\mathrm{GHz}$ waveguide to finally be radiated. In addition, to achieve greater decoupling between both layers, the radiating field will also be orthogonal by rotating one layer $90^{\circ}$ with respect to the other. Fig 3.55 shows the concept of stacked 
cylindrical cavities removing the nails in the contour of the cell. It is observed that the two ports are fed at different points rotated $90^{\circ}$.

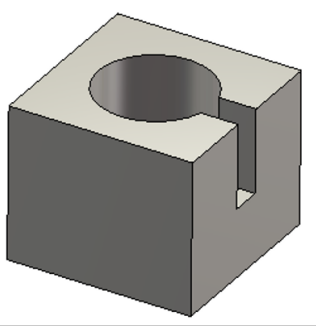

30-GHz Cavity

Layer 0

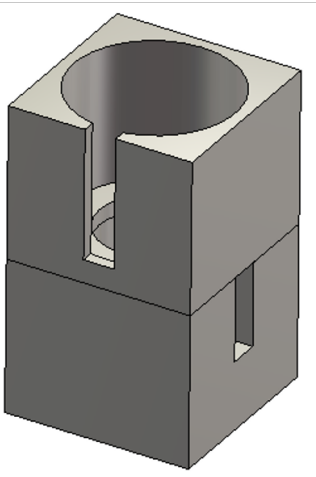

20-GHz Cavity

Layer 1

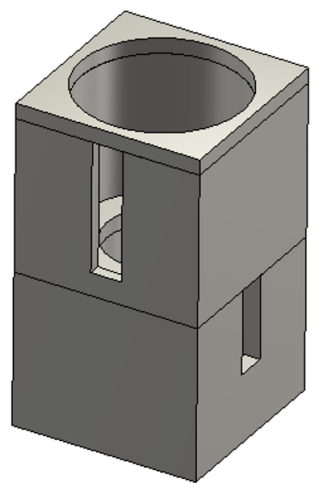

Radiating Aperture

Layer 2

Figure 3.55: Layers of the dual-band unit cell from bottom to top.
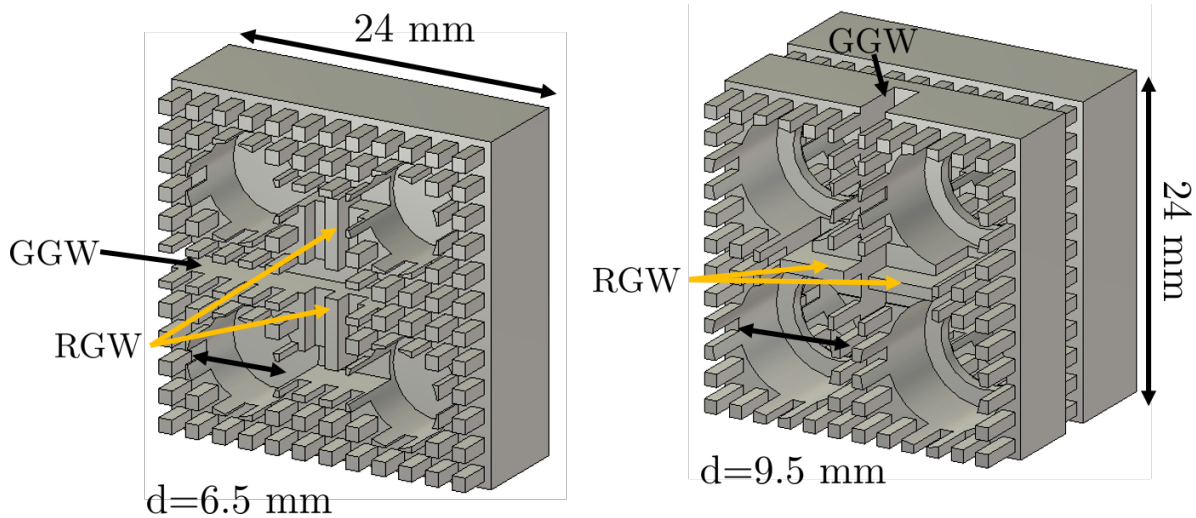

Figure 3.56: Perspective view of layer 0 and layer 1 of the dual-band antenna.

In Figs. 3.56 and 3.57, the $2 \times 2$ subarray is shown. All dimensions in each layer must be consistent with the corresponding working frequency. Therefore, the nails of the lower layer have a height of a quarter wavelength at $30 \mathrm{GHz}$, which is $2.5 \mathrm{~mm}$. In the upper layer, working at $20 \mathrm{GHz}$, the height of the nails is $3.75 \mathrm{~mm}$. The nail width and periodicity are equal in both cases for ease of design. The distance between apertures is $0.8 \lambda_{0}$ at $20 \mathrm{GHz}$ and $1.2 \lambda_{0}$ 


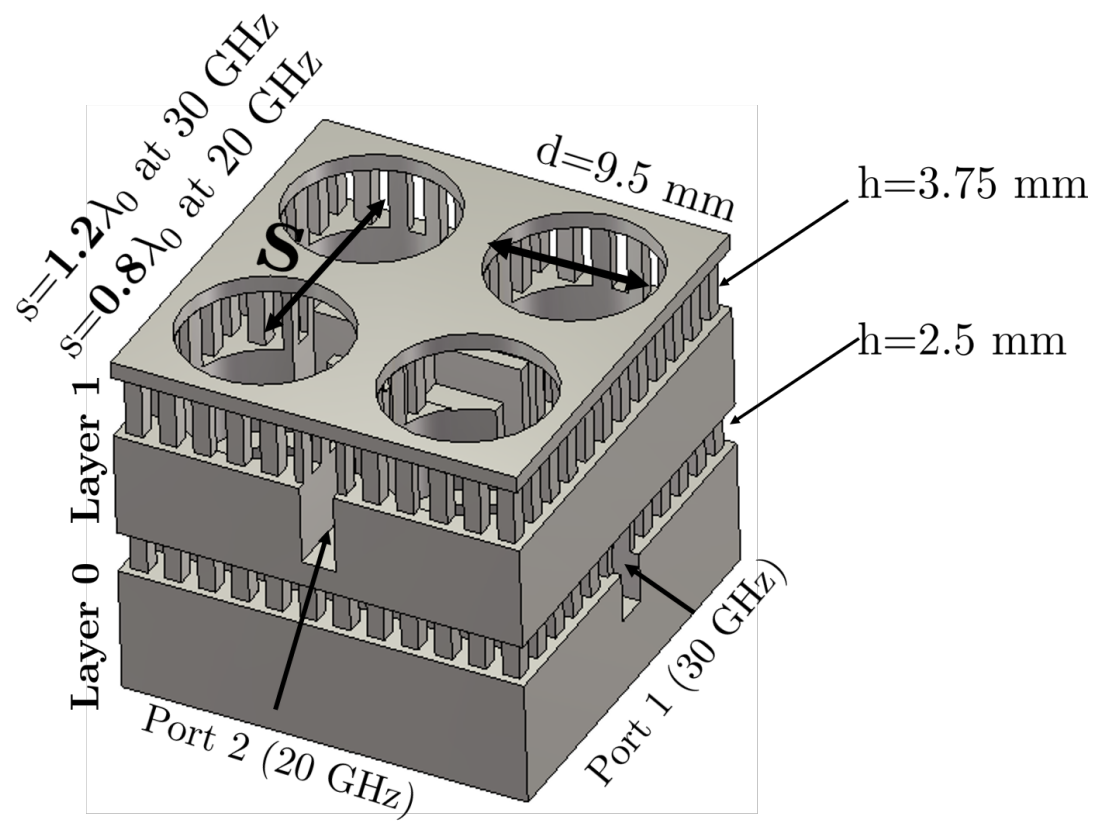

Figure 3.57: $2 \times 2$ dual-band array antenna.

at $30 \mathrm{GHz}$. This separation between apertures would lead to grating lobes at $30 \mathrm{GHz}$ but they will be mitigated by using a waffle-grid placed on top of the antenna. More details will be given in Chapter 4 .

In Fig 3.58, the S-parameters of the $2 \times 2$ subarray are represented. Fig. $3.58 \mathrm{a}$ shows the reflection coefficient of the antenna in the $20-\mathrm{GHz}$ band. The simulated $\mathrm{S}_{11}$ is below $-10 \mathrm{~dB}$ within a bandwidth from $19.5 \mathrm{GHz}$ to $22 \mathrm{GHz}$, widely covering the K-band typically used in SATCOM applications. On the other hand, Fig. 3.58b shows the $\mathrm{S}_{11}$-parameter at the $30 \mathrm{GHz}$ band. A bandwidth of $11 \%$ is observed below $-15 \mathrm{~dB}$. Finally, Fig. 3.58c represents the $S_{21}$ parameter between both ports, confirming the expected high isolation. 


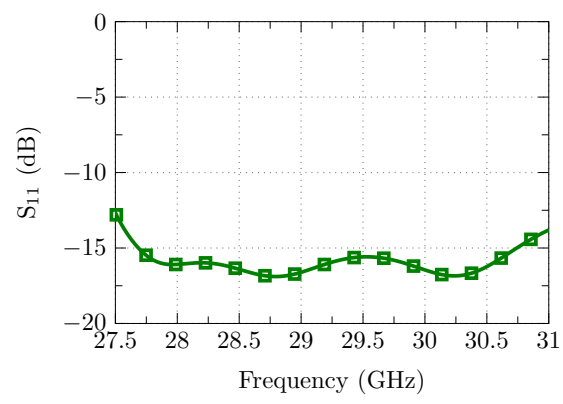

(a)

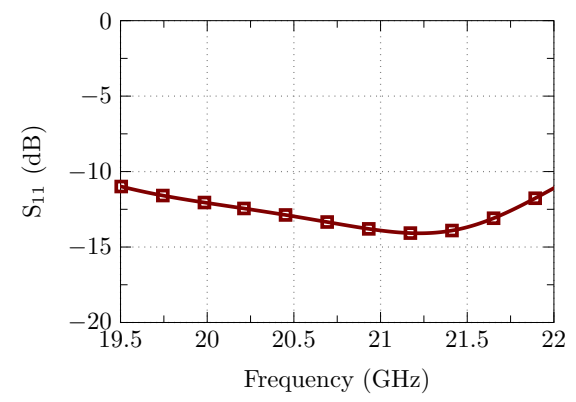

(b)

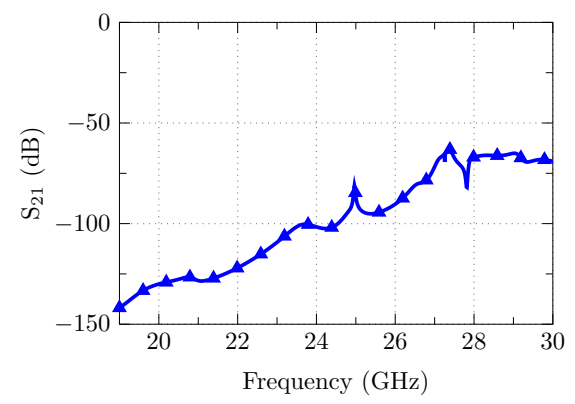

(c)

Figure 3.58: S-parameters of the $2 \times 2$ dual-band array antenna: (a) $\mathrm{S}_{11}$ (Port 1); (b) $\mathrm{S}_{11}$ (Port 2); (c) $\mathrm{S}_{21}$. 
CHAPTER 3. RADIATING ELEMENTS FOR GAP WAVEGUIDE ARRAYS 


\title{
Chapter 4
}

\section{Gap Waveguide Array Antennas}

\author{
"You can't connect the dots \\ looking forward; you can only \\ connect them looking backwards. \\ So you have to trust that the dots \\ will somehow connect in your \\ future."
}

- Steve Jobs

This Chapter brings together the concepts that have been detailed in Chapters 2 and 3. Gap Waveguide Arrays will be presented here. In most cases, it has been possible to carry out not only the design but also the manufacture and measurement of the prototypes. The feasibility and performance of the antennas and their possible applications will be studied.

Firstly, a $16 \times 16 \mathrm{~V}$-band antenna with circular polarization is presented. The wide frequency band available, over $7 \mathrm{GHz}$ of continuous spectrum, makes the V-band very desirable for applications requiring high transmission bandwidth such as indoor and outdoor communications.

The other prototypes were developed to operate at 20 or $30 \mathrm{GHz}$, aiming at future applications in satellite communications on the move. Different prototypes have been designed in the Ka-band, linearly-polarized (LP), dualpolarized (DP), circularly-polarized (CP) and dual-band (DB) array antennas have been considered. Several prototypes have been manufactured using different manufacturing techniques such as CNC milling or 3-D printing. These antennas provide desirable features for SATCOM such as lightness, low profile 


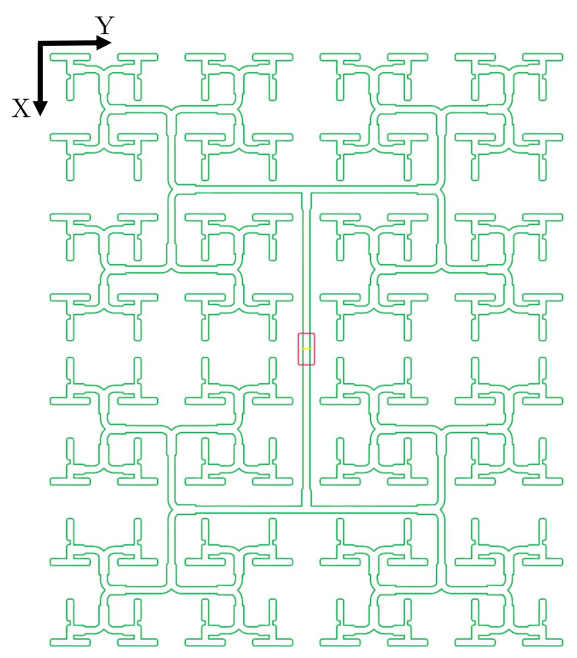

(a)

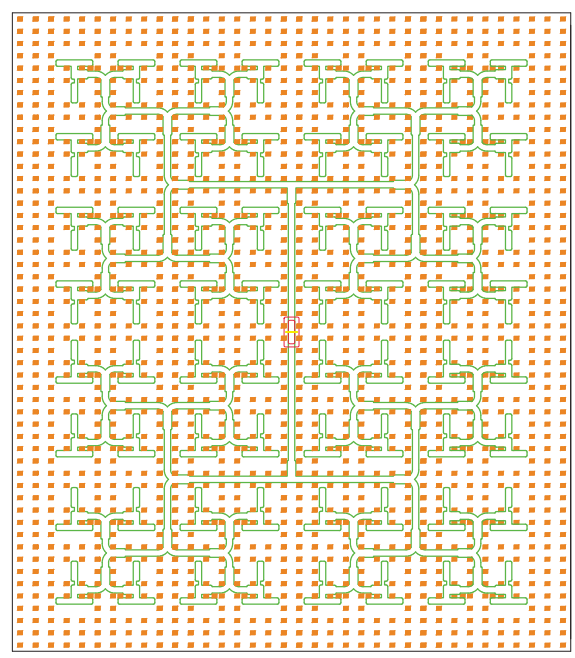

(b)

Figure 4.1: E-plane GGW corporate-feeding network: (a) without and (b) with the nails surrounding the network.

and high efficiency. In addition, the use of GW ensures good assembly and avoids leakage.

\section{1 $16 \times 16$ Single-Layer Array Antenna in V- band}

The $60 \mathrm{GHz}$ band has become very attractive due to the huge unlicensed bandwidth available worldwide [229]. One of the challenges for mm-wave wireless systems is to develop highly directive planar antennas with good aperture efficiency. Expected low cost of manufacturing process for gap waveguide technology, compared to hollow waveguide array antennas at mm-wave frequency bands can be a considerable advantage. Among the recent contributions, those carried out in Chalmers Antenna Group stand out [68],[72],[74],[191], as it has already been discussed in previous chapters. Nevertheless, most of these antennas use a feeding network composed of two layers. Here, a more compact $16 \times 16 \mathrm{~V}$-band antenna in Gap Waveguide is proposed.

The full array consists on 64 unit cells as described on Section 3.1.2. The basic cells are fed by a 1 to 64 corporate network designed with GGW. Splitters use step discontinuities to achieve the proper matching, like that in the unit 


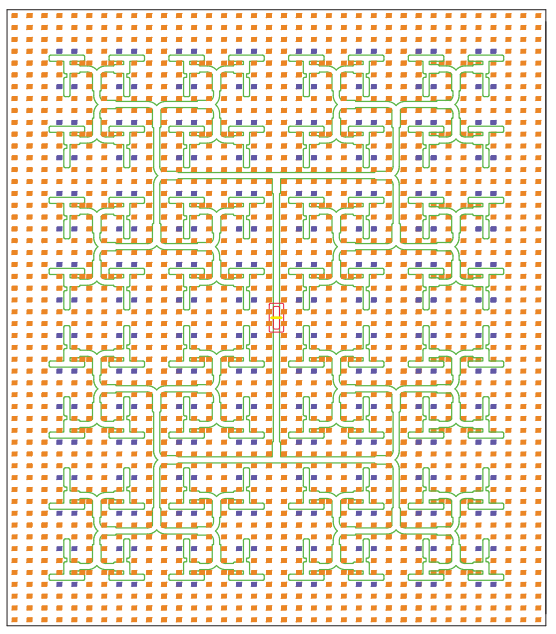

(a)

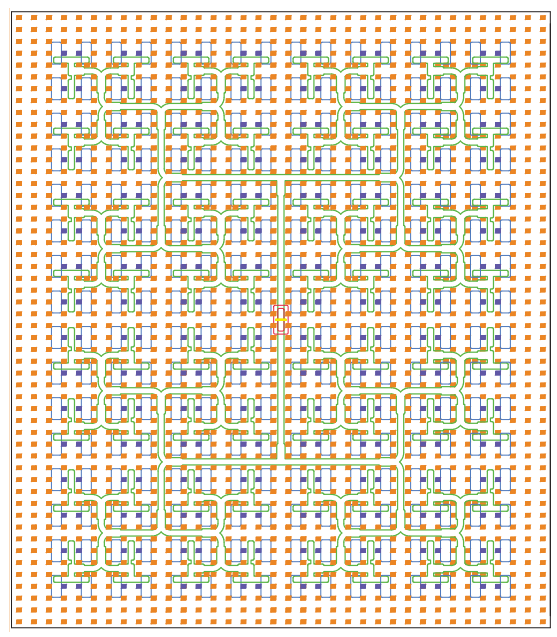

(b)

Figure 4.2: (a) Full corporate networks and cavities. Coaxial nails depicted in purple. (b) Apertures (blue) are added over the network.

cell. In Figs. 4.1 and 4.2 a top view of the layers that makes up the antenna is presented. First, 4.1a shows the 1 to 64 corporate-feed distribution network. This distribution network is made with an E-plane GGW. As it was already explained, this network allows a more compact distribution of the signal. At the end of the network, it is necessary to add one extra divider on one of the sides to compensate the phase at those output ports. In $4.1 \mathrm{~b}$, the nails of the bed are highligthed in orange. All nails have the same height and width. The horizontal and vertical periodicity is slightly different, though. The spacing is larger in the horizontal dimension because enough room must be left to place the apertures. As for the slots, the ratio $W_{\text {slot }} / L_{\text {slot }}$ was optimized to get the best possible impedance bandwidth. However, this ratio should not exceed 0.5 because to avoid cross polarization [72]. In our case, this ratio is set to $W_{\text {slot }} / L_{\text {slot }}=0.46$, so a trade-off between getting the desired frequency bandwidth and not worsening the crosspolar component is reached.

Then, in Figs. 4.2a and 4.2b, the shortened-nails (purple) are emphasized for the sake of clarity. The shortened-nails are responsible for creating the coaxial cavities. Finally, the apertures (blue) are placed on top. Therefore the antenna is hosting both the distribution network and the radiating cavities in a single layer.

The antenna is fed from the backside. Feeding from one side would reduce the complexity of the design but it would spoil pattern symmetry. The feeder 
is in fact a key part for good performance. A transition must be designed from the WR-15 to the GGW. It is not a straigthforward transition since the field must be divided at the very input port. In Fig. 4.3 the fundamental parameters of the transition are shown.

\section{Input transitions from WR-15 to GGW}

A transition connects a standard WR-15 waveguide (port of the V-band array antenna), to two GGW (initial stage of the feeding network). Fig. 4.4 depicts top and back views of the transition. As can be observed, the transition is an $\mathrm{H}$-plane power divider with a central slit to balance the power evenly to both branches.

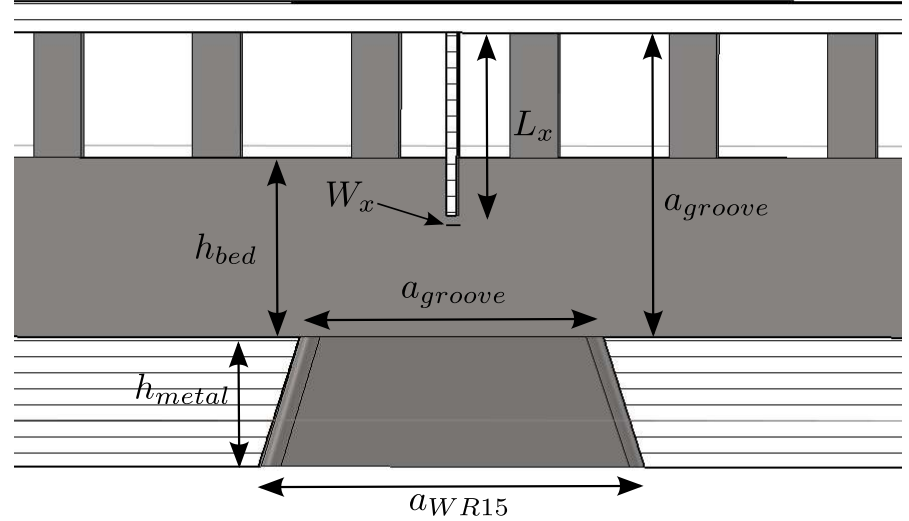

Figure 4.3: Cross-section of the RW-to-GGW transition. The values of the basic design parameters are presented in Table 4.1 .

Table 4.1: Optimized dimensions of the RW-to-GGW transition

\begin{tabular}{|c|c|}
\hline Parameter & Value $(\mathbf{m m})$ \\
\hline $\mathrm{W}_{x}$ & 0.1 \\
\hline $\mathrm{L}_{x}$ & 1.7776 \\
\hline $\mathrm{h}_{\text {pin }}$ & 1.2195 \\
\hline $\mathrm{a}_{W R 15}$ & 3.7592 \\
\hline $\mathrm{b}_{W R 15}$ & 1.8796 \\
\hline $\mathrm{a}_{\text {groove }}$ & 2.9561 \\
\hline $\mathrm{b}_{\text {groove }}$ & 0.8 \\
\hline
\end{tabular}




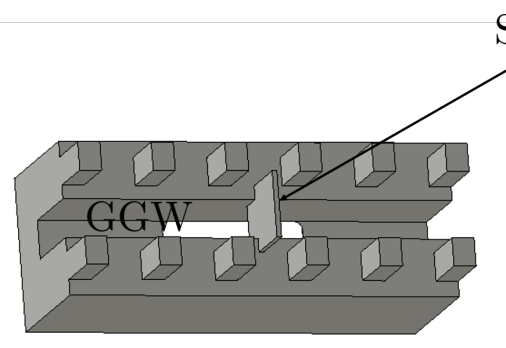

Front View
Slit

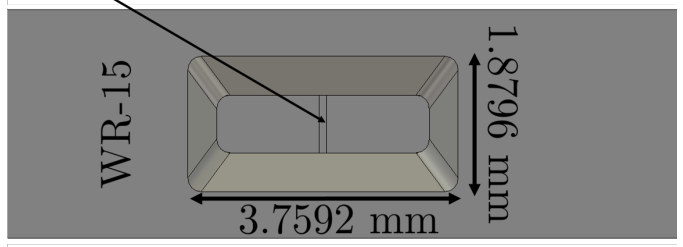

Back view

Figure 4.4: (Left) RW-GGW transition without a lid where the slit is observed. (Right) Back view of the transition

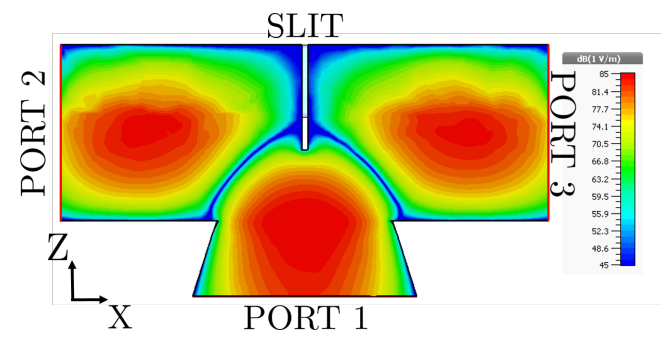

Figure 4.5: Magnitude of the electric field within the GGW transition.

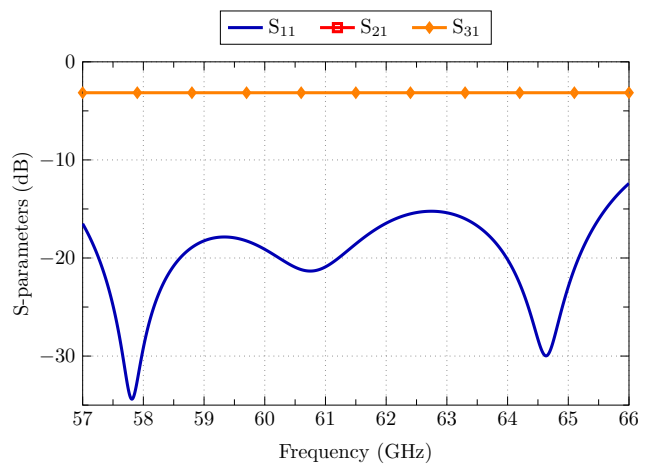

Figure 4.6: Simulated S-parameters of the RW-to-GGW transition. 


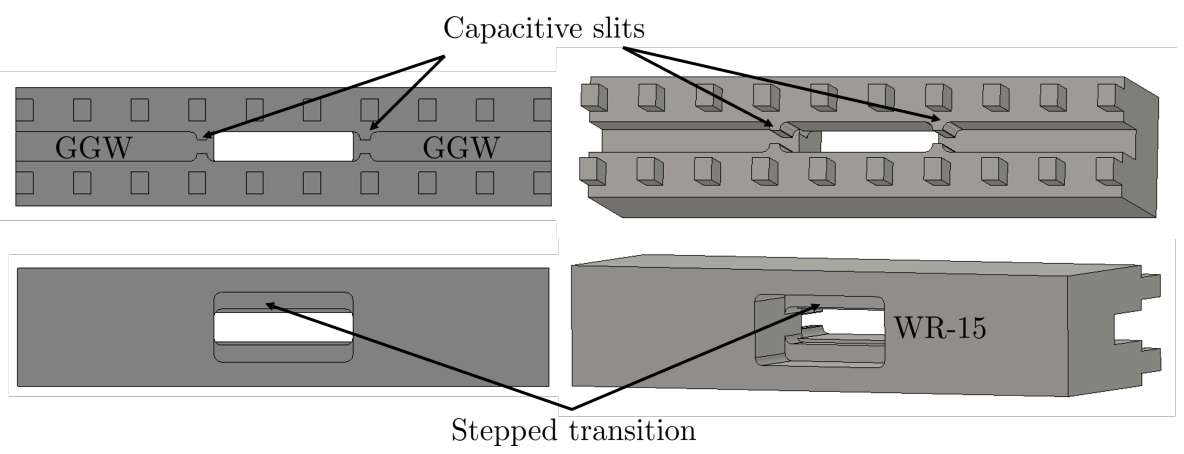

Figure 4.7: RW-GGW transition using capacitive slits.

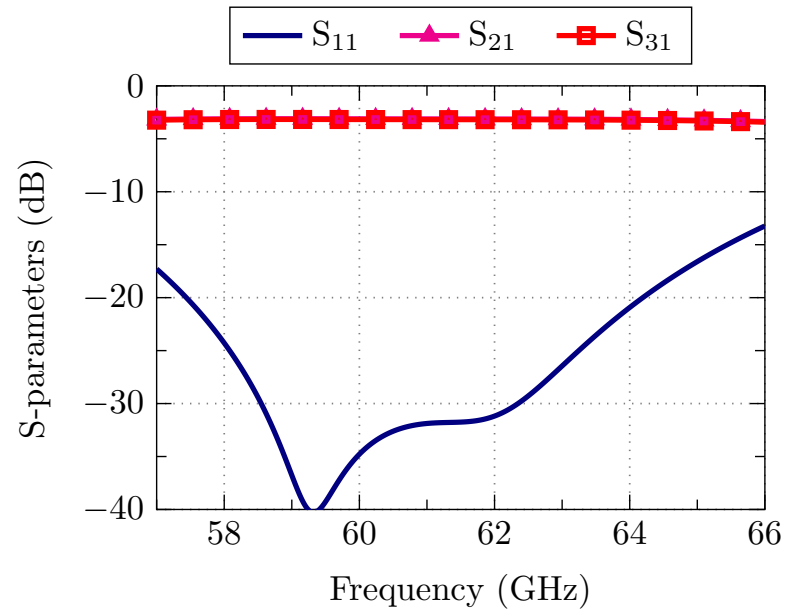

Figure 4.8: Simulated S-parameters of a RW-to-GGW transition using capacitive slits.

The dimensions of the WR-15 standard waveguide are $3.7592 \times 1.8796 \mathrm{~mm}^{2}$, whereas the inner dimensions of the GGW are $2.9561 \times 0.8 \mathrm{~mm}^{2}$. Note that the GGW presents one very narrow dimension due to the closeness to the final stages of the corporate feeding network (see Fig. 4.1a). Such a difference in dimensions forces us to use a tapered transition between both waveguides, as illustrated in Fig. 4.3. This tapering is more convenient than a stepped transition since, at V-band, steps needed would be of the order of microns. Such accuracy would be somehow hard to guarantee using a conventional milling process. The transition has been optimized separately from the rest of the antenna to speed up the design process. The optimization process has been mainly focused on 
tuning depth $\left(L_{x}\right)$ and width $\left(W_{x}\right)$ of the central slit, which have a substantial effect on the matching to the input impedance. The optimized values of the transition parameters are included in Table 4.1 .

Fig. 4.5 displays the electric field on XZ-plane of the RW-to-GGW transition at $61.5 \mathrm{GHz}$. As can be observed, the use of a slit in the central part of the transition is enough to divide the power into both sides efficiently. The main drawback of this system, however, is that, the width must be as thin as possible $(0.1 \mathrm{~mm})$ for proper performance. In the $\mathrm{V}$-band, this value is not critical, but, maybe, this strip would be barely tens of microns wide at higher frequencies, which would complicate fabrication process. The simulated S-parameters of the transition are shown in Fig. 4.6. As can be seen, the RW-to-GGW transition presents identical transmission parameters, $S_{21}$ and $S_{31}$, while $S_{11}$-parameter is below $-15 \mathrm{~dB}$ within a $15 \%$ relative bandwidth.

As an alternative, a second transition from WR-15 to GGW was designed. This transition (Fig. 4.7) eliminates the central slit and uses capacitive windows in the GGW instead. They are placed on both sides of the input waveguide. Sparameters (Fig. 4.8) are similar to that of the first transition. The advantage is that it can also be used at frequencies above $60 \mathrm{GHz}$ since the size of the capacitive windows is less sensitive to assembly and fabrication issues.

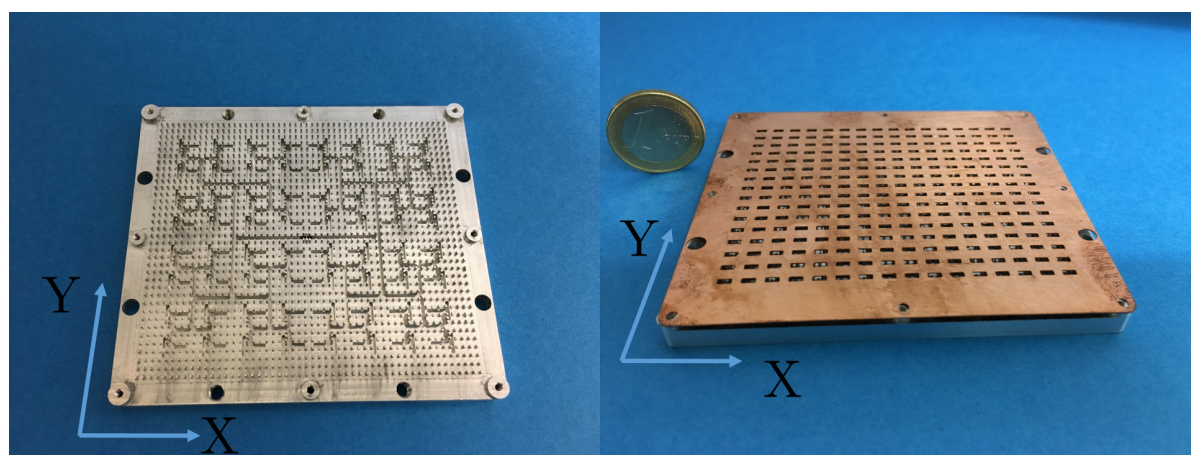

Figure 4.9: Manufactured Linear-Polarized Antenna in the V-band. (Left) Corporate-feeding network. (Right) Copper plate with the apertures.

\section{Experimental results of the Linearly-polarized antenna}

The antenna was manufactured in the facilities of the electromagnetic radiation group at iTEAM using a milling machine. The prototype is shown in Fig. 4.9. The array was designed taking into account the inner rounded corners with a maximum radius of $200 \mu \mathrm{m}$ due to restrictions on the dimensions of the milling tools. The slots of the antenna are also designed with rounded corners to have 
a real manufacturing environment. The spacing of the apertures in the $\mathrm{x}$ direction is $0.92 \lambda_{0}$, and $0.78 \lambda_{0}$ in the y-direction. Simulated E-plane radiation pattern is shown in Fig.4.10a. Sidelobe level remains below $-10 \mathrm{~dB}$ for the entire bandwidth. However, grating lobes become significant only at the upper end of the frequency band. At $66 \mathrm{GHz}$, the electrical spacing between slots is $\lambda_{0}$ in the E-plane. The simulated H-plane pattern is shown in Fig.4.10c. In this case, the radiation patterns do not exhibit those high grating lobes at 66 $\mathrm{GHz}$ because they are canceled due to the radiation pattern of the slot itself. Both cases present excellent cross polarization suppression, better than $60 \mathrm{~dB}$.

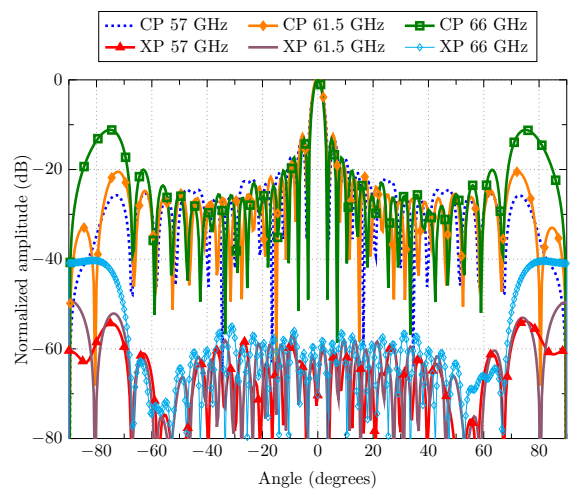

(a)

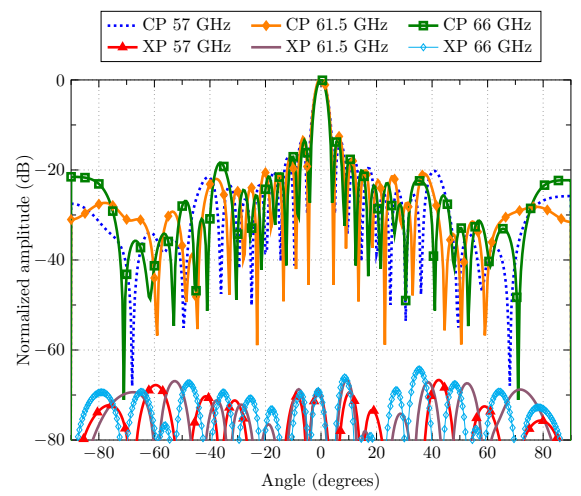

(c)

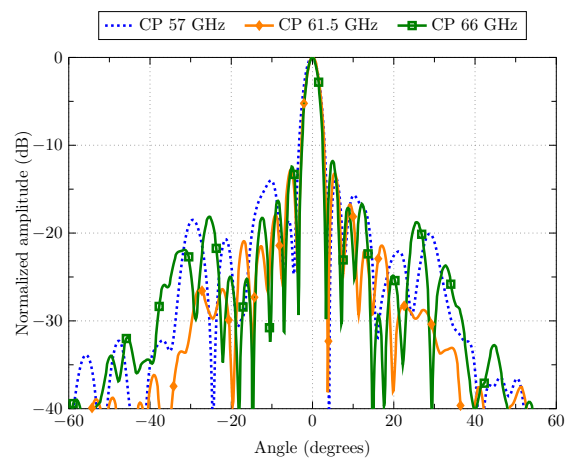

(b)

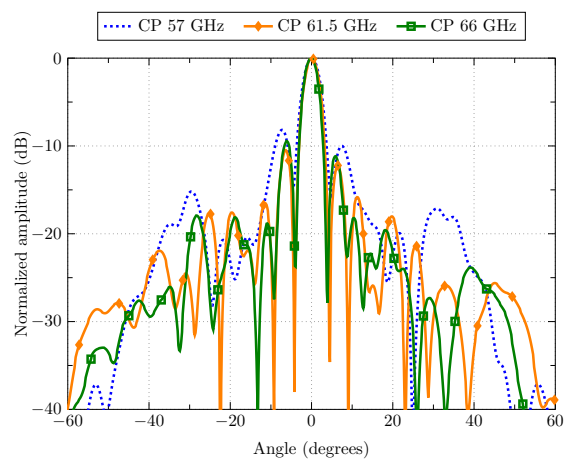

(d)

Figure 4.10: For several frequencies : (a) Simulated E-plane pattern. (b) Measured E-plane pattern. (c) Simulated H-plane pattern. (d) Measured Hplane pattern. 


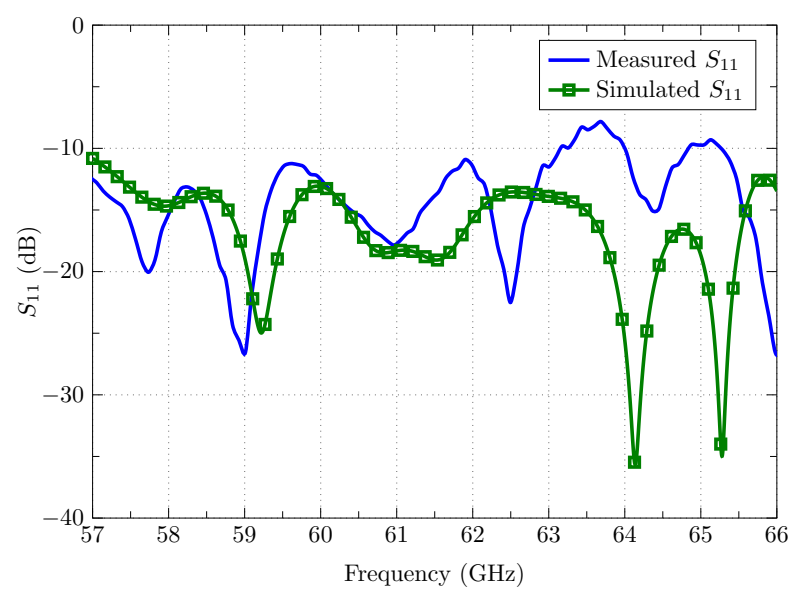

Figure 4.11: Measured and simulated reflection coefficient of the linearlypolarized antenna

In the right column of Fig. 4.10, the measured radiation patterns for both main cuts are shown. The large bandwidth of the distribution network allows a great stability of the patterns for the entire bandwidth, from $57 \mathrm{GHz}$ to 66 $\mathrm{GHz}$. However, a slight asymmetry in the radiaton patterns is observed. This is due to two reasons. First of all, there is some uncertainty in the alignment of the antenna in the anechoic chamber and secondly, there is a slight warping of the copper plate containing the slots. A thicker plate would solvable it easily.

Regarding the reflection coefficient, a comparison between simulation and measurement is presented in Fig. 4.11. It can be observed that there is a good agreement of both curves for the lower part of the band, but not for the upper part. However, the $\mathrm{S}_{11}$ remains close to the target of $-10 \mathrm{~dB}$ over the entire band.

Finally, after manufacturing and measurement, a metrological study was made. Some discrepancies between the designed and manufactured model were observed. A deviation of $150 \mu \mathrm{m}$ on the depth of the groove was found. Grooves depth is fundamental to control $\beta_{w g}$ and probably it is one of the main reasons for not obtaining a better reflection coefficient.

\section{Polarizer}

As it is well known, circular polarization may be very advantageous for certain applications. For that reason, a polarizing layer was designed to seamlessly convert the linear into circular polarization, keeping the same array as a feeder. In [230] a metal cavity mounted on the slot was proposed to achieve a circularly- 


\section{CHAPTER 4. GAP WAVEGUIDE ARRAY ANTENNAS}
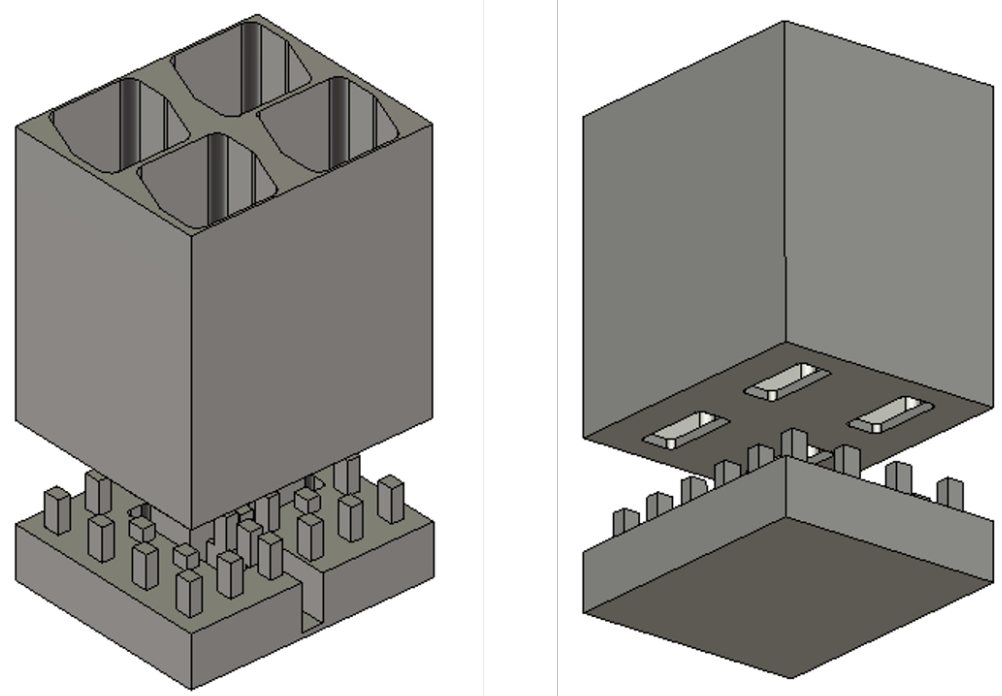

Figure 4.12: 3D sketch of the unit cell with the polarizer.
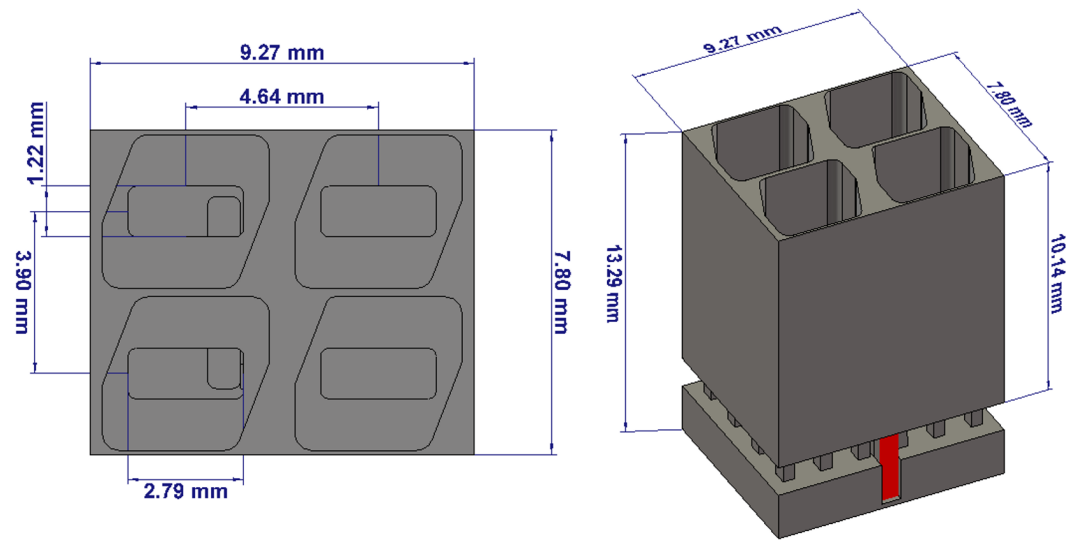

Figure 4.13: Different sketches showing the dimensions of the polarizer.

polarized antenna. Setting the height and the cross-section of the cavity, two orthogonal modes with equal amplitude but with a phase-shift of $90^{\circ}$ can be obtained. Here the same concept is used to transform LP into CP. Neither the cavities nor the slots dimensions have been changed with respect to the linearly-polarized array antenna. 


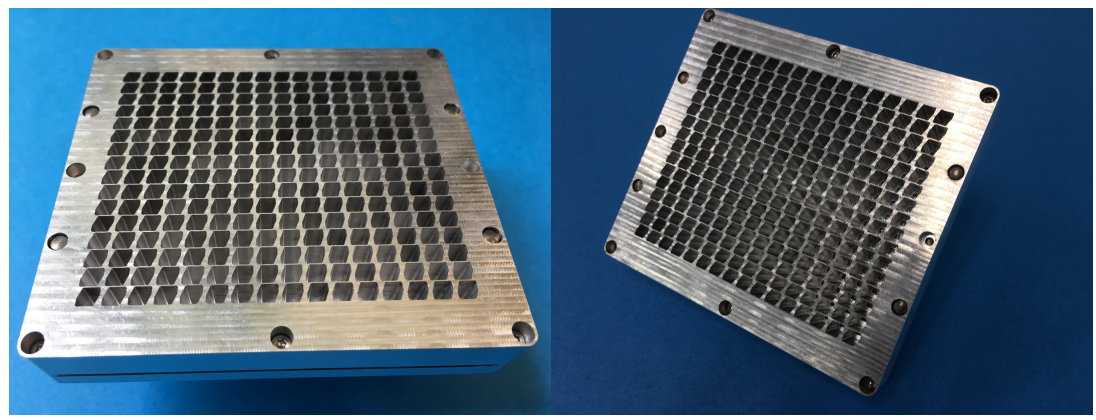

Figure 4.14: Manufactured $16 \times 16$ polarizer in the $\mathrm{V}$ band.

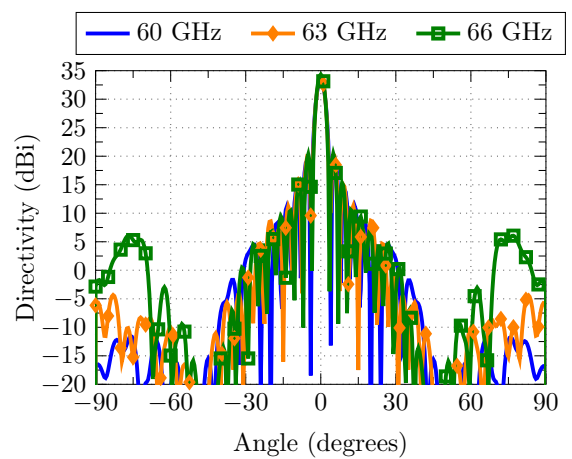

(a)

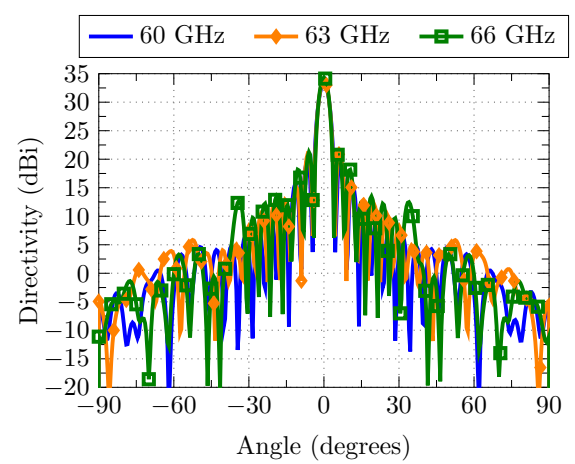

(b)

Figure 4.15: For several frequencies : (a) Simulated YZ-plane pattern. (b) Simulated XZ-plane pattern.

The bed of nails with the E-plane splitter is the same as previously. Above the bed of nails, the new radiators are located. The height of the metal cavity is key in order to get a good polarization purity. The cross-section could be designed in different ways, always under the premise of getting a phase shift of $90^{\circ}$ between two orthogonal modes. Here, a chamfered square is used. In Fig. 4.12 two images of the unit cell are shown. In the new piece, the slots are integrated directly with the polarizer as drawn in the right subfigure. By using a single piece of metal as radiating layer, possible air gaps are avoided. Fig. 4.13 shows a top view of the RHCP radiating element where the cross-section is observed. In those figures the most relevant dimensions of the polarizer have been included. The manufactured polarizer is shown in Fig. 4.14. 


\section{CHAPTER 4. GAP WAVEGUIDE ARRAY ANTENNAS}

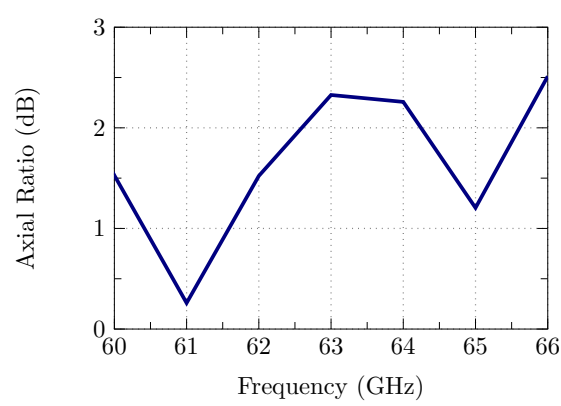

(a)

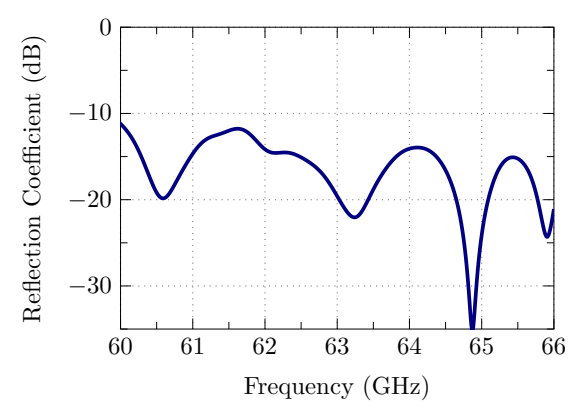

(b)

Figure 4.16: (a) Simulated Axial Ratio. (b) Simulated $\mathrm{S}_{11}$ parameter for the circularly-polarized array.

Simulated radiation patterns presented in Figs. 4.15a and 4.15b demonstrate that the grating lobes are not a significant problem even at $66 \mathrm{GHz}$, the highest operation frequency. Interestingly, that wide aperture spacing in the x-direction is compensated by the element radiation pattern. However, the higher the working frequency, the higher the grating lobes in the YZ-plane.

Nevertheless, it was not a simple task to achieve good polarization purity in a bandwidth as large as defined above. The working band is from $60 \mathrm{GHz}$ to $66 \mathrm{GHz}$, which is almost $10 \%$. The preliminary axial ratio and reflection coefficient are presented in Fig. 4.16. The axial ratio remains below $2.5 \mathrm{~dB}$ over the entire bandwidth, being better in the lower part of the band. Regarding the $\mathrm{S}_{11}$-parameter, the simulation shows a value below $-12 \mathrm{~dB}$ from 60 to 66 $\mathrm{GHz}$.

\section{Conclusions}

One distinctive feature of this antenna is the possibility to alternate the lids for linear or circular polarization, using the same bed of nails. Unfortunately, measured results of the circularly-polarized antenna are not available so far. Yet, measurements on the linearly-polarized antenna verify the potential of the GW coaxial cavities. In addition, the GGW feeding network has enabled a compact array design in a single-layer architecture. 


\section{2 $8 \times 8$ Single-Layer Cavity-Backed Slot Array Antenna}

The following prototype, as well as all successive ones, work on Ka-band. From now on this will be the band of interest in order to develop novel antennas for SATCOM applications on the move. Therefore, the goal is to design lighter and more compact that can compete with current solutions. This first antenna has two main differences with respect to the previous one, beyond the change in frequency from $\mathrm{V}$ to Ka band. Firstly, the antenna is not fed by a GGW distribution network. Now the signal is distributed corporately through a combination of GGW and RGW. The advantages of this second type of network have already been discussed in previous chapters.

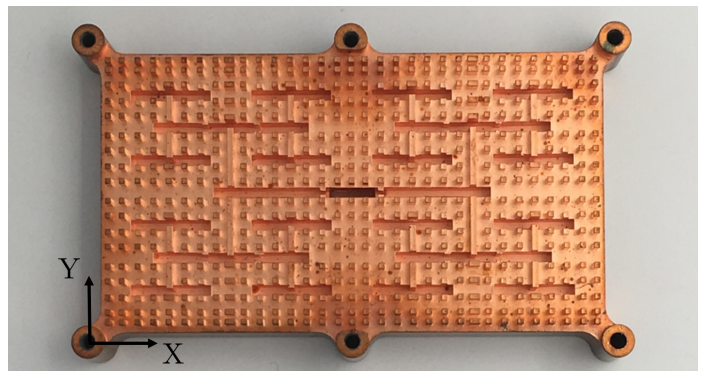

(a)

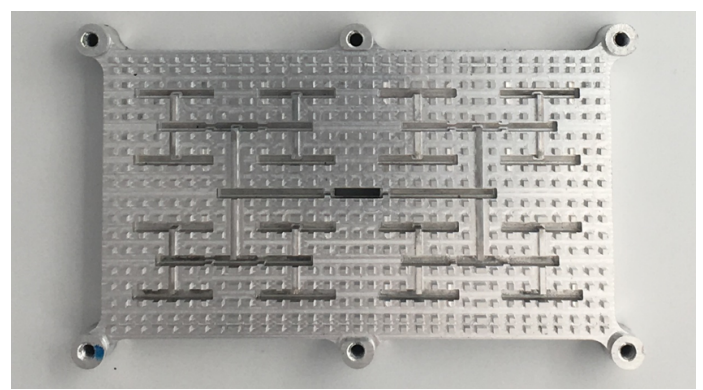

(b)

Figure 4.17: Antenna prototypes. They are uncovered to clearly show the feeding network and cavities in GW technology. Both antennas are identical and share the same radiating layer. (a) Prototype 1: 3D printed. (b) Prototype 2: Machined aluminum [231].

In addition, a study of performance of different fabrication techniques has been carried out on this model. Two identical antennas has been fabricated 


\section{CHAPTER 4. GAP WAVEGUIDE ARRAY ANTENNAS}

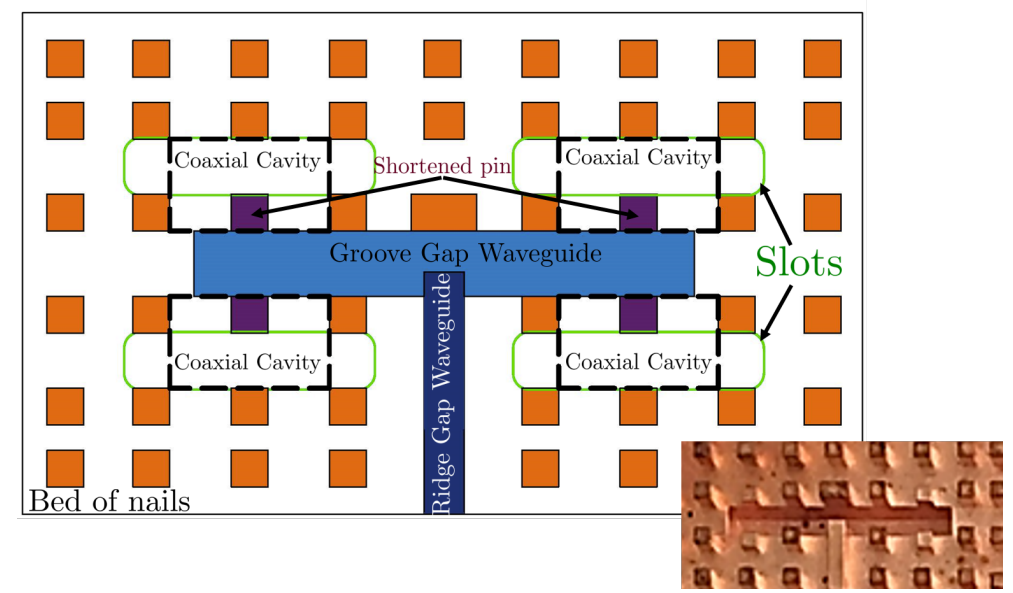

Figure 4.18: Depiction of the basic radiating element. The most important parts that composed the cell are detailed. The slots, colored in green, are located above the coaxial cavities which are framed in a black dashed line [231].

using CNC Milling and 3D printing (Fig. 4.17). The design decisions taken to get an $8 \times 8$ single-layer antenna are explained next.

\section{Basic radiating cell}

The $2 \times 2$-element subarray represents the basic radiating structure of the proposed gap waveguide antenna. To simulate an array environment, periodic boundary walls are placed in the external region of the basic radiating element to take into account the mutual coupling in an ideal infinite 2-D array. Firstly, the corporate-feeding network and the coaxial cavities are presented in Fig 4.18. Next, the four elements in the subarray are coupled to the branches of a combined groove-ridge gap waveguide corporate-feeding networks as explained in chapter 3. Nail height in the coaxial cavities is $h_{s}=1.35 \mathrm{~mm}$, the rest of the nails exhibit the same height ( $\lambda / 4$ at the central operating frequency), $h_{p}=2.44$ $\mathrm{mm}$.

The nails are not distributed in an even grid but this does not affect the bandgap of the textured surface. The non-uniformity is done on purpose. On the one hand, the periodicity of the nails is wider in the central part of the unit cell, thus facilitating possible tolerance problems during subsequent in-house milling tasks (prototype 2). In the surroundings of the unit cell, on the other hand, the periodicity of the nails can be narrower thanks to the absence of grooves. In the central part, nails are spaced $2.4 \mathrm{~mm}$ and $2.5 \mathrm{~mm}$ on the $\mathrm{X}$ and $\mathrm{Y}$ axis, respectively. In the surroundings, $2.1 \mathrm{~mm}$ and $1.5 \mathrm{~mm}$, respectively. 
The 16 cells comprising the complete antenna are identical to the one shown in Fig. 4.18.

\section{Radiating layer}

Two identical feeding layers have been manufactured, one with 3D-printing and the other with a CNC Milling technique. However, to be able to adequately compare the experimental results, it is convenient to have the least number of uncertainties. For this reason it was decided that both antennas would share the same radiating layer. This radiating layer was manufactured on the same milling machine as the second prototype. Some ribs have been included to provide greater consistency to this layer. This solution is better than making a thicker radiating layer which would lead to a noticeable increase in weight in larger arrays. Finding a trade-off between weight, height and performance, a good choice is to use a ribbed grid. Fig. 4.19 shows a conventional slots plate on the left, and on the right the proposed radiating layer including ribs.

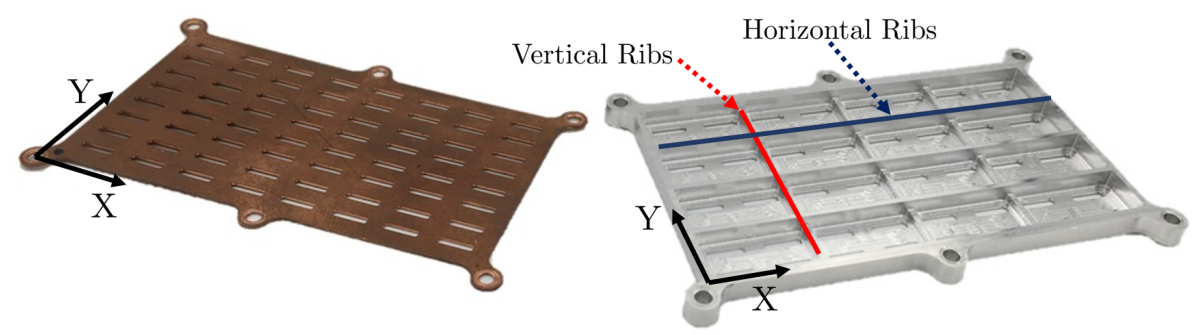

Figure 4.19: Radiating layers. The one on the left corresponds to a conventional slots plate $(h=0.5 \mathrm{~mm})$. On the right, a ribbed grid $\left(h_{\max }=3.5 \mathrm{~mm}\right)$ is added.

Adding these ribs, the metal sheet can hardly be bent. Nevertheless, this solution presents some issues to be solved. The $y$-component of the electric field will be forced to be null on the vertical rib walls. This produces a nonuniform aperture illumination, which degrades the radiation pattern in the H-plane (XZ). Such degradation is not observed in the E-plane (YZ) since the electric field is normal to the horizontal ribs. A quick and effective solution is simply to reduce the height of the vertical ribs in order to achieve a more uniform illumination of the antenna. Therefore the height of the ribs is set to $3 \mathrm{~mm}$ horizontally and $1 \mathrm{~mm}$ vertically.

As shown in Fig. 4.20, if vertical ribs were $3 \mathrm{~mm}$ in height (left figure), field nulls would appear along the $y$-dimension on the vertical rib walls. On the other hand, if the height of the vertical ribs is reduced to $1 \mathrm{~mm}$ (right figure), such effect is partly alleviated, leading to a more uniform illumination. This problem does not occur in the YZ-plane (horizontal ribs) because the field is 


\section{CHAPTER 4. GAP WAVEGUIDE ARRAY ANTENNAS}
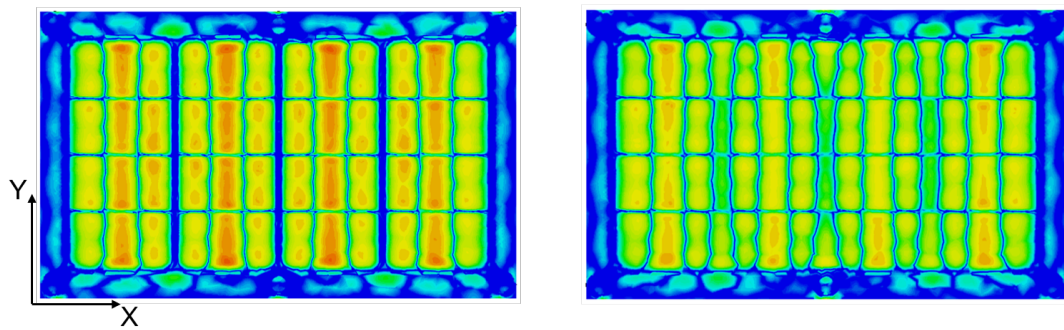

Figure 4.20: Electric field $E_{y}$ across the apertures. On the left figure using single-height grid. On the right, two-heights grid, being vertical ribs shorter than horizontal ones [232].

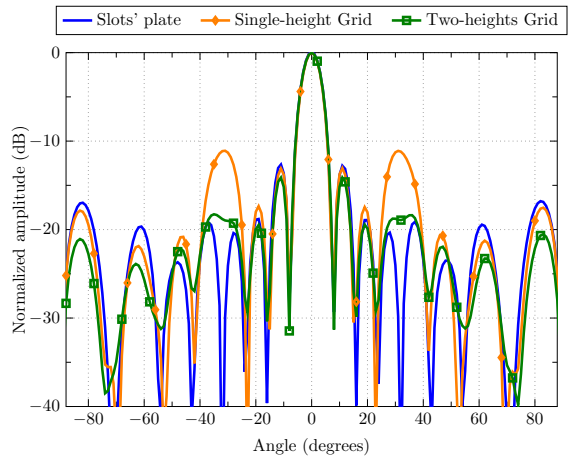

(a)

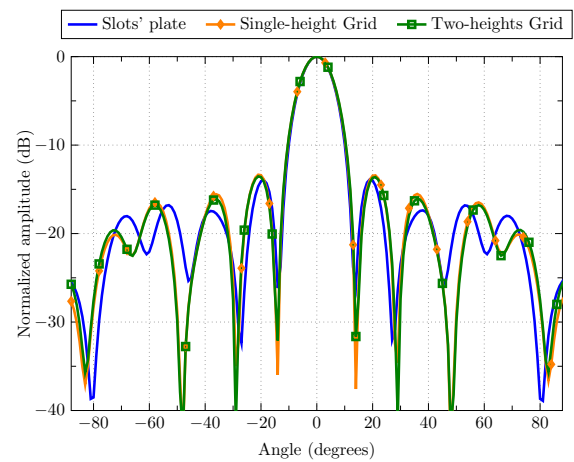

(b)

Figure 4.21: Radiation patterns using different configurations in the radiating layer [232]: (a) XZ-plane @ $30 \mathrm{GHz}$. (b) YZ-plane @ $30 \mathrm{GHz}$.

normal to the horizontal rib walls. The amount of metal added is minimal and the weight of the antenna is not greatly altered. Its effect is clearly seen in the behavior of the radiation patterns (Fig. 4.21). First, it is observed how the E-plane pattern is immune to these changes in height. This is not the case, however, for the H-plane pattern, where it is clearly observed that if the height of the grid is not lowered, side lobes would increase considerably.

\section{Experimental results}

As explained above, the study presented here is aimed at assessing the viability of additive manufacturing by comparing its electrical performance with milling. Both antennas are shown in Fig. 4.17 without the top plate. The first prototype 
4.2 $8 \times 8$ Single-Layer Cavity-Backed Slot Array Antenna

Table 4.2: Measured antenna parameters of 3D-printed prototype.

\begin{tabular}{|c|c|c|c|c|c|c|}
\hline Frequency $(\mathrm{GHz})$ & 28.5 & 29 & 29.5 & 30 & 30.5 & 31 \\
\hline \hline Directivity $(\mathrm{dBi})$ & 24.82 & 24.5 & 25.42 & 26.03 & 25.87 & 25.72 \\
\hline Gain $(\mathrm{dBi})$ & 23.96 & 23.89 & 24.45 & 25.55 & 25.15 & 24.58 \\
\hline Antenna Efficiency (\%) & 82.0 & 87.01 & 80.08 & 89.62 & 84.84 & 77.04 \\
\hline
\end{tabular}

Table 4.3: Measured antenna parameters of aluminum prototype.

\begin{tabular}{|c|c|c|c|c|c|c|}
\hline Frequency $(\mathrm{GHz})$ & 28.5 & 29 & 29.5 & 30 & 30.5 & 31 \\
\hline \hline Directivity $(\mathrm{dBi})$ & 25.39 & 25.58 & 25.85 & 26.09 & 26.24 & 26.22 \\
\hline Gain (dBi) & 24.48 & 24.43 & 24.82 & 25.64 & 25.40 & 25.31 \\
\hline Antenna Efficiency (\%) & 81.17 & 76.74 & 78.89 & 90.02 & 82.26 & 81.08 \\
\hline
\end{tabular}

has been manufactured using SLA printing, followed by copper plating. SLA is a laser-based technology which uses an UV-sensitive liquid resin. A UV laser beam scans the surface of the resin and selectively hardens the material corresponding to the object's cross section, thus building the $3 \mathrm{D}$ body from the bottom up. The required supports for constructing overhangs and cavities, are automatically generated and manually removed at the end of the process.

Once the solid object is built, it is subject to a metallic coating process whose outcome is critical for the final electrical performance. In fact, techniques such as physical vapour deposition or electroplating, require access to every internal surface to correctly plate the structure [233]-[234]. As can be inspected in Fig. 4.17a, the targeted 3D structure was conceived to be free of intricate tunnels and allows a proper copper deposition by a straightforward procedure. The 3D-printed version was manufactured by Protolabs company. Conversely, the second prototype has been fully built inhouse by making use of the well-known CNC milling technique. This process requires a milling machine, aluminum material and several cutting bits, which must be equal to or smaller than the smallest feature of the desired pattern.

Both antennas have been measured under the same environment and during the same period of time, to avoid imbalances in the measurement equipment and calibration. The measured radiating parameters of both antennas are shown in Tables 4.2 and 4.3. The mean efficiency is above $80 \%$ for both prototypes within the band of interest, being that of the 3D-printed version slightly higher. Recall that conductivity of copper is better than that of aluminum. Fig. 4.22 shows the radiation patterns in the main cuts ( $\mathrm{E}$ and $\mathrm{H}$ plane) for several frequencies. In each subfigure, the patterns of the $3 \mathrm{D}$-printed and aluminum antennas, in green and yellow, respectively, are compared. All the measured patterns present an overall good agreement except for one visible 


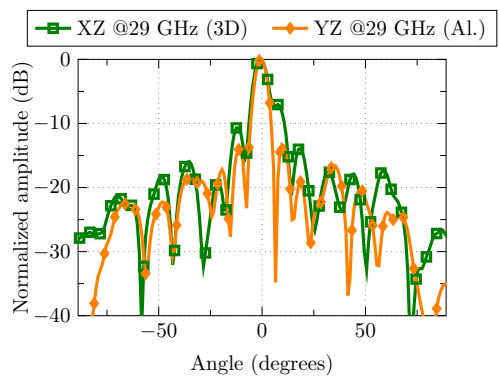

(a)

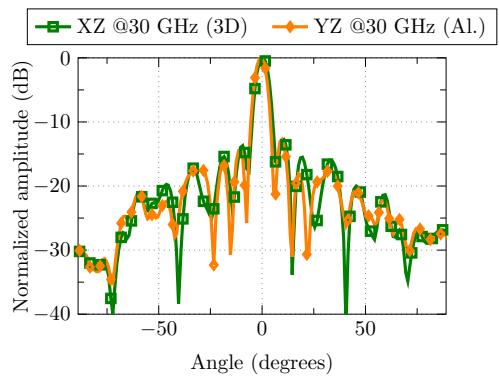

(c)

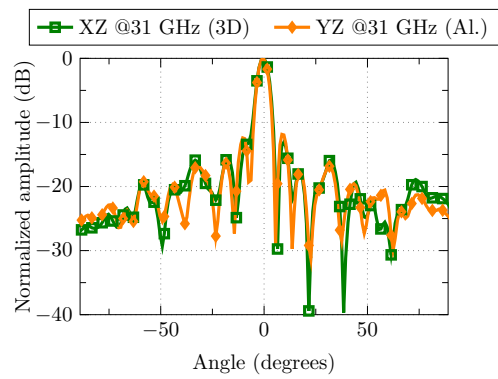

(e)

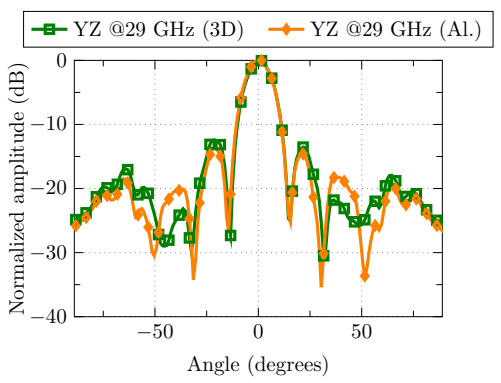

(b)

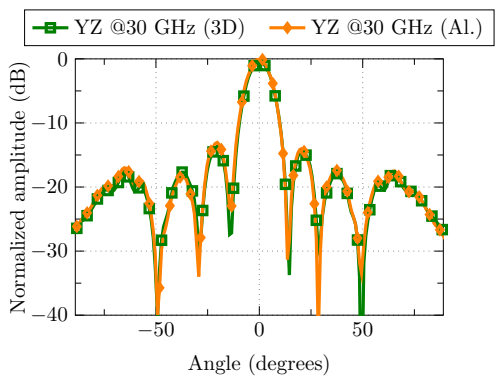

(d)

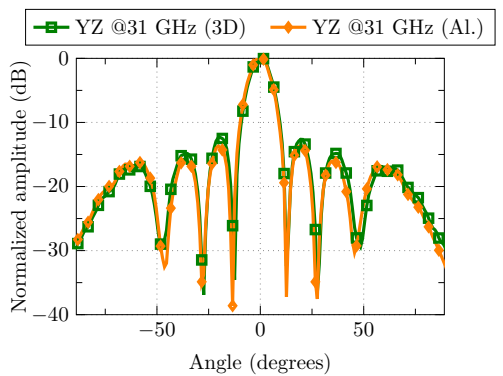

(f)

Figure 4.22: Measured radiation patterns of both prototypes (aluminum and 3D-printed) at several frequencies: (a) H-plane at $29 \mathrm{GHz}$; (b) E-plane at 29 $\mathrm{GHz}$; (c) H-plane at $30 \mathrm{GHz}$; (d) E-plane at $30 \mathrm{GHz}$; (e) H-plane at $31 \mathrm{GHz}$; (f) E-plane at $31 \mathrm{GHz}[231]$.

discrepancy at $29 \mathrm{GHz}$ in the $\mathrm{H}$ plane. Finally, the magnitude of the measured reflection coefficient is plotted in Fig. 4.23. A good agreement is shown between 


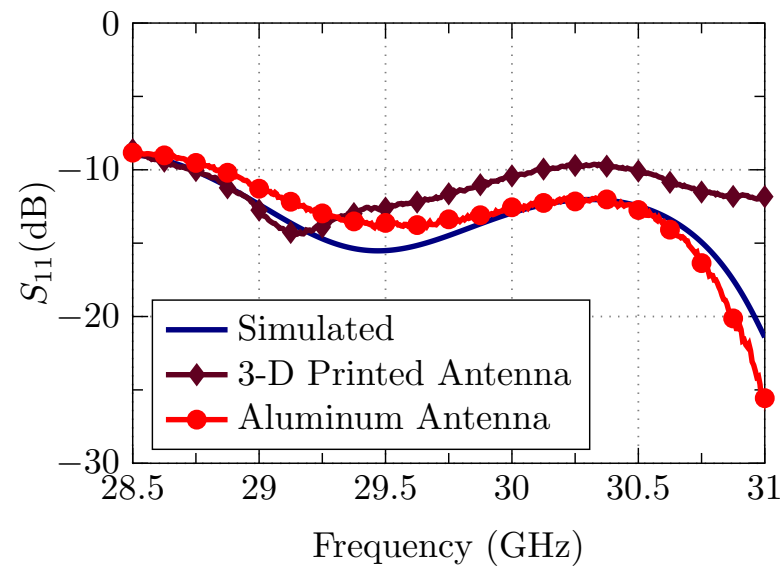

Figure 4.23: Measured input reflection coefficient for both antennas [231].

Table 4.4: Metrological study of antenna dimensions.

\begin{tabular}{|c|c|c|c|}
\hline \multirow{2}{*}{ Parameter } & \multirow{2}{*}{ Designed (mm) } & \multicolumn{2}{|c|}{ Mean deviation $(\mu \mathrm{m})$} \\
\hline & & Aluminum & 3D printing \\
\hline Groove width & 1.6 & 7 & 37 \\
\hline Groove depth & 3.473 & 13 & 33 \\
\hline Ridge width & 0.986 & 13 & 14 \\
\hline Ridge height & 1.548 & 41 & 44 \\
\hline Nail width & 0.9 & 15 & 11 \\
\hline Nail height & 2.439 & 3 & 9 \\
\hline Shortened-nail width & 0.9 & 7 & 7 \\
\hline Shortened-nail height & 1.35 & 12 & 23 \\
\hline
\end{tabular}

both experimental results, despite the aluminum version reveals a greater similarity to the simulated result.

\section{Discussion between 3D printing and CNC manufacturing}

A metrological study was carried out on both antennas to analyze the fabrication accuracy. The measured deviation from ideal dimensions of different constitutive parameters are shown in Table 4.4. Predictably, the analysis of the tabulated data reveals that the mean manufacturing deviation committed by the milling technique is lower than that observed for the 3D-printed antenna.

Figs. $4.24 \mathrm{~b}, 4.24 \mathrm{~d}$ and $4.24 \mathrm{f}$ show three zoomed pictures of the 3D-printed antenna. Several imperfections in the sampled GGWs are evidenced. Such 


\section{CHAPTER 4. GAP WAVEGUIDE ARRAY ANTENNAS}

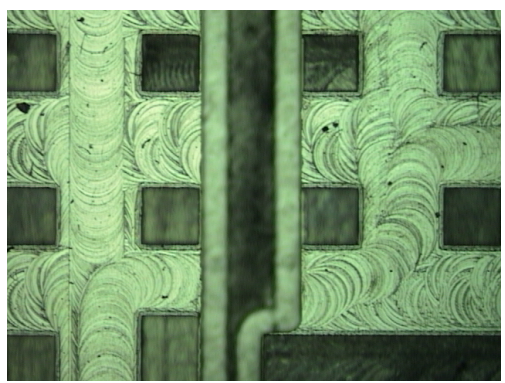

(a)

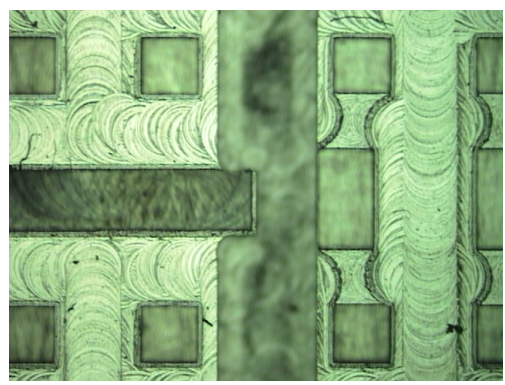

(c)

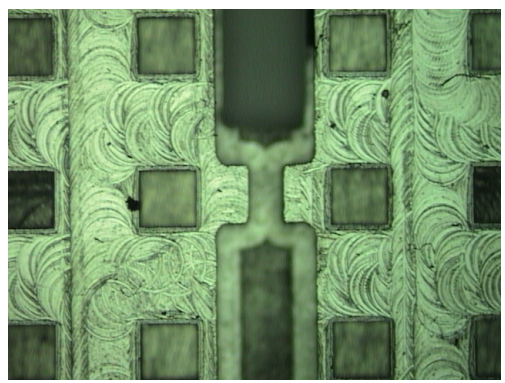

(e)

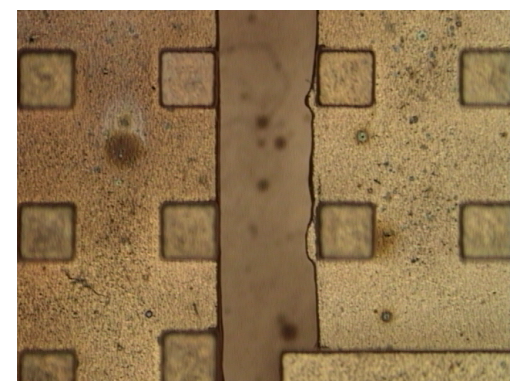

(b)

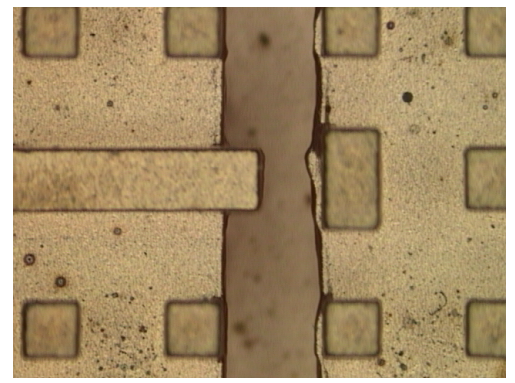

(d)

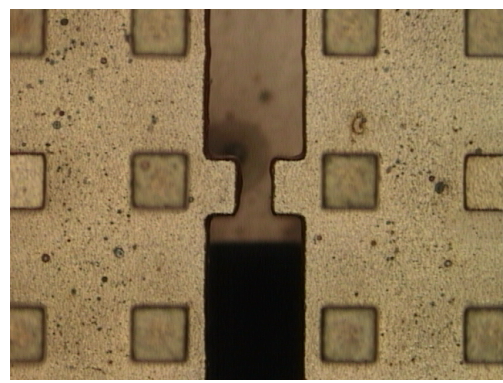

(f)

Figure 4.24: Zoomed sampled views of the prototypes [231]: (a), (c), (e) aluminum antenna; (b), (d), (f) 3D-printed antenna.

inaccuracies in the waveguides' walls were detected throughout the antenna. Conversely, those defects are not present in the aluminum piece, where the circular ripple left by the milling cutter is visible instead (see Figs. 4.24a, 4.24c and $4.24 \mathrm{e}$ ). Such ripple, however, is merely one micron thick and its effect on electrical performance is negligible. One might state, therefore, that the overall 
fabrication quality of the aluminum antenna is notably better than that observed for the 3D-printed prototype. This fact contradicts to a certain extent what it was affirmed in [193], where larger roughness was observed in the aluminum version. It must be pointed out that the aluminum breadboard in [193] was subject to a sanding process, which is undoubtedly responsible for such roughness. As it is already suggested in [193], an eventual finishing process for aluminum pieces may lead to additional roughness which significantly increases the conductor losses. In our study, the ohmic losses are similar in both prototypes as it is evidenced from the measured antenna efficiency. Note that no finishing process has been applied here. On the other hand, 3D printing technique inherently avoids the typical rounded corners of milled structures.

\section{Conclusions}

Making the same array using two very different manufacturing techniques has allowed us to validate and appreciate the advantages and disadvantages of each of them. Milling remains the most conservative and safe technique. The manufacturing tolerances at these frequencies are not critical and the experimental results behave as expected. However, it is an expensive technique, and hardly acceptable for mass production. Additive manufacturing, on the other hand, still has certain accuracy limitations, however, it is much cheaper than milling and also allows lighter prototypes. The experimental measures achieved with the additive manufacturing prototype have shown it as a reliable technique.

\section{3 $4 \times 4$ Single-Layer Circularly-Polarized Array Antenna}

The next antenna makes use of the chamfered cylindrical cavities explained in Chapter 3. As explained, these cavities radiate circular polarization in a very compact way by hosting the feeding network and the polarizer in the same layer. So, the basic radiating element and the feed network are put together here to build a $4 \times 4$ array (Fig. 4.25). The size chosen is considered large enough to demonstrate how these two structures can be combined in an array, being the extension to larger arrays formally straightforward. The apertures are uniformly spaced and surrounded by the corporate feeding network combining RGW and GGW.

Fig. 4.26 shows the final 3D layout before manufacturing. Input section and one of the cavities have been zoomed in for clarity. The antenna input is located at the back surface, making use of a transition to a WR-28 standard waveguide. The lack of room left by the corporate network and the apertures forces to excite the RGW by a reduced cross-section waveguide. Hence, a stepped transition to 


\section{CHAPTER 4. GAP WAVEGUIDE ARRAY ANTENNAS}

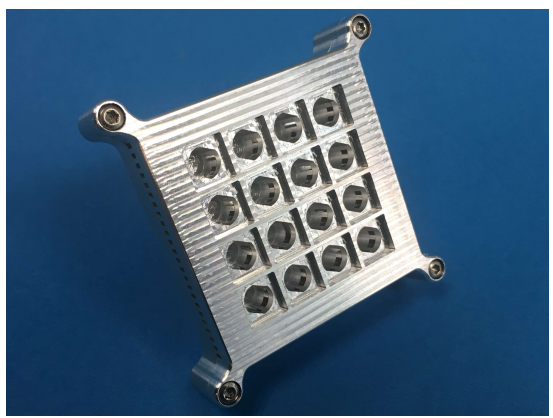

(a)

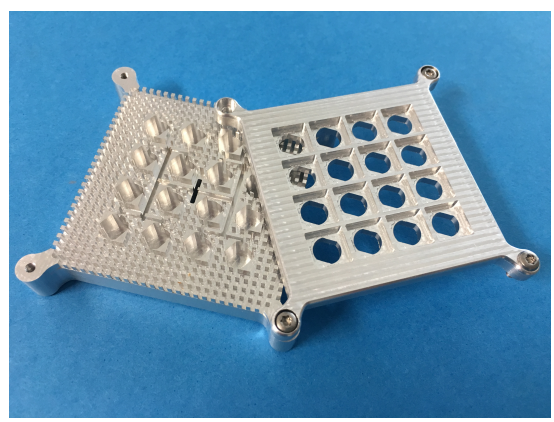

(b)

Figure 4.25: (a) Perspective view of the manufactured antenna. (b) Antenna array prototype [235].

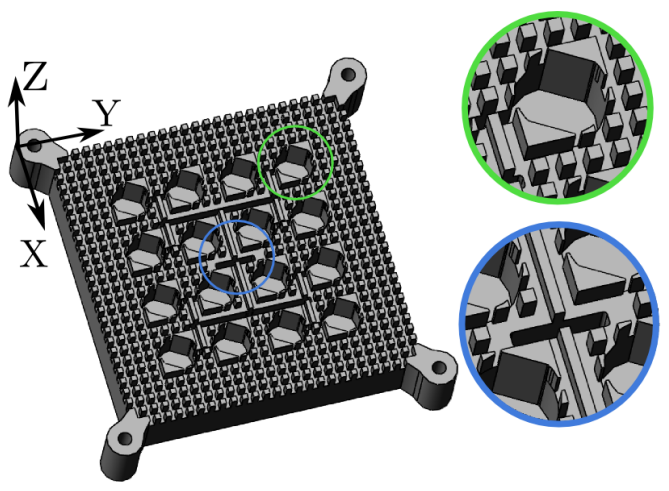

Figure 4.26: $3 \mathrm{D}$ model of the $4 \times 4$ antenna. Detailed views of one radiating cavity and the input transition are enlarged [235].

a WR-28 waveguide has been designed separately. It was found that one single step was enough to achieve good impedance matching. A cross-sectional view of the transition from WR-28 standard waveguide to RGW is shown in Fig. 4.27. Regarding the dimensions of the textured surface, the nail height and width, $h_{p}$ and $l_{p}$, respectively, as well as the nail periodicity, $p_{p}$, are the following: $h_{p}=2.5 \mathrm{~mm}, l_{p}=1 \mathrm{~mm}$ and $p_{p}=2 \mathrm{~mm}$. The band-gap performance with these values, goes from $22 \mathrm{GHz}$ to $53 \mathrm{GHz}$, which broadly covers the required frequency range. This is certainly far more bandwidth than necessary, but it 


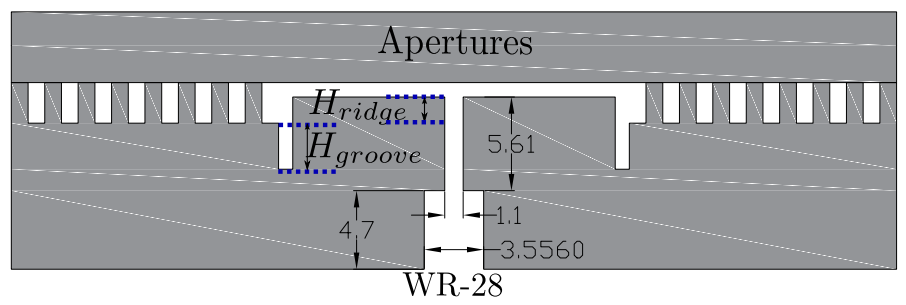

Figure 4.27: Cross-sectional side view of the $4 \times 4$ antenna. A complete view from the WR-28 input port to the first GGW, passing through the input RGW, can be seen. Most relevant dimensions of the WR-28 to RGW transition are shown [235].

can also be convenient. A wide stopband may be advantageous if, for example, a frequency shift occurs due to inaccuracies in nails manufacturing. In such case the operation frequency band would still be within the bed of nails' stopband.

\section{Radiating Layer: Waffle grid}

Given the size of the chamfered holes and the room required by the waveguide feeding network within the bed of nails environment, the apertures have been spaced as close as possible, i.e., $10 \mathrm{~mm}$ in our case, as illustrated in Fig. 4.28a. Such array spacing corresponds to $1.03 \lambda_{0}$ at the maximum frequency of the intended band, $31 \mathrm{GHz}$, therefore grating lobes will still be present. To alleviate their effect, a waffle grid has been added to the lid. The waffle grid frames each chamfered aperture into a larger square aperture. If an appropiate waffle height is chosen, both fundamental degenerate modes are properly excited at the square apertures. Hence, such grid preserves the circular polarization while increasing the effective aperture area of the unit cell. This field filling effect at the aperture level leads to a significant reduction in grating lobe level, as shown in Fig. 4.28b. Sidelobes at $\theta=70^{\circ}$ are lowered by more than $5 \mathrm{~dB}$. Notice that the thickness of the waffle grid itself is $2 \mathrm{~mm}$ only, a reasonable value in terms of the total antenna height. Finally, Fig. 4.29 shows the aperture electric field at different heights, where the uniform excitation of the array elements can be noticed.

\section{Manufacturing process}

The described antenna has been fully manufactured and measured inhouse. For manufacturing, a Datron M-25 CNC Milling Machine has been used. Machine tolerances and milling restrictions influenced some of the dimensions finally chosen. In particular, groove depth and width are constrained by the milling 


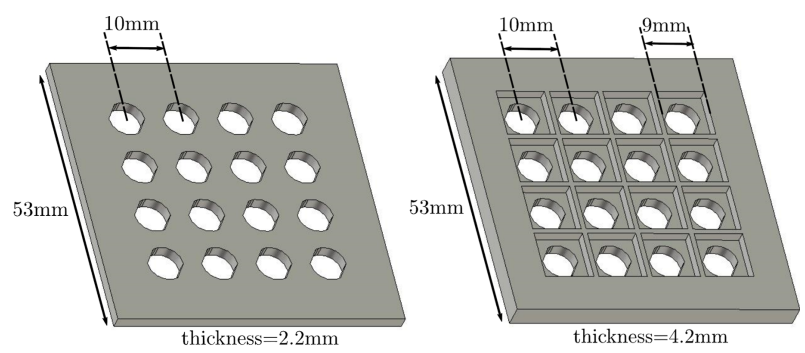

(a)

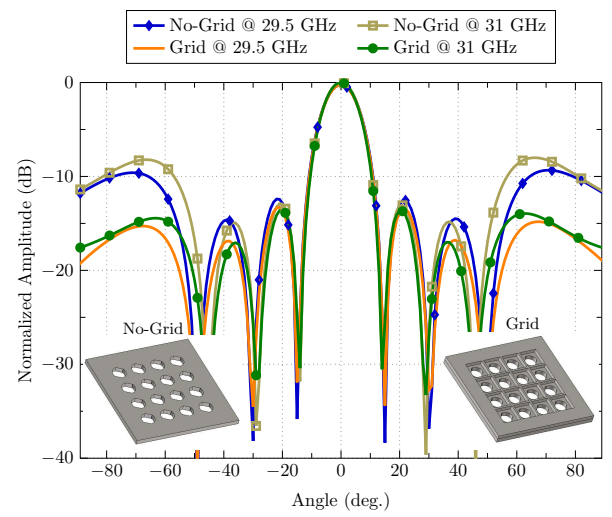

(b)

Figure 4.28: (a) Antenna lid showing the chamfered apertures (left) and antenna lid with the waffle grid framing the original apertures (right). (b) Radiation patterns with and without grid. Patterns are at the lower and upper frequency of the band (XZ-plane) [235].

tools available. Notice that the deeper the groove, the wider the tool must be. Grooves cannot be made as deep and narrow as one would wish. Given the operation band, a groove depth of at least $6 \mathrm{~mm}\left(h_{p}+H_{\text {groove }}\right)$ is required, which forced us to use a milling tool of $0.4 \mathrm{~mm}$ in diameter. Thicker tools would damage groove walls due to unavoidable vibrations.

Despite these fabrication precautions, a couple of errors during the milling process were detected. The first one was caused by inconsistencies of the CNC milling machine, while the other can be attributed to a milling software bug. The most serious one was a hole perforated onto one of the input ridges. It was decided to try to fix this pitfall by filling the wrong drill with a conducting paste (LPKF ProConduct). 

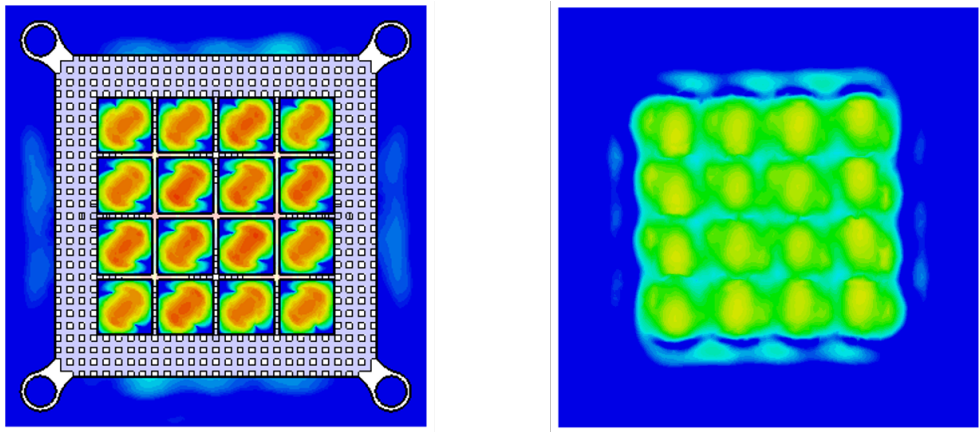

Figure 4.29: Electric fields distribution for two different heights. (Left) Inside the Waffle-Grid. (Right) Above the Waffle-Grid.

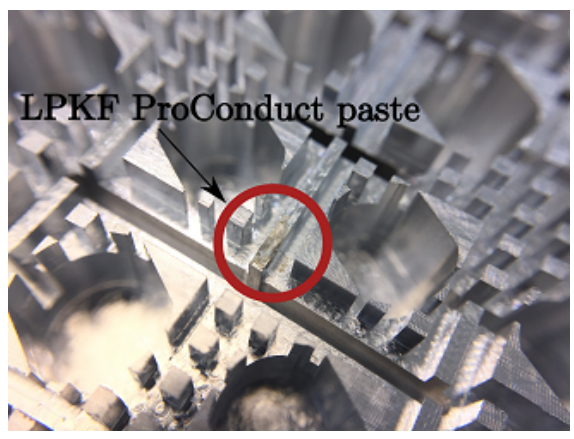

(a)

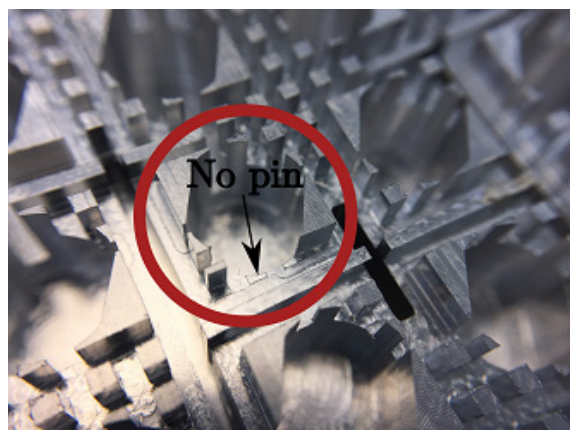

(b)

Figure 4.30: (a) Picture of the RGW where the drilled hole has been covered by the LPKF ProConduct paste. (b) Detail of the sector where a nail was incorrectly milled out [235].

Fig. 4.30a shows the fixed hole covered with the paste. The second error detected was related to a missing nail in one of the cavities, apparently swept away by the machine. In Fig. $4.30 \mathrm{~b}$ it can be seen that the tool overdrilled a metallic part between the input ridge and one cavity, removing one nail and part of the cavity chamfer. Since only one row of nails is used to isolate the ridge from the cavities, that missing nail played a key role. Unfortunately, this error could not be fixed and its effect will manifest itself as pattern asymmetries along the XZ plane. 


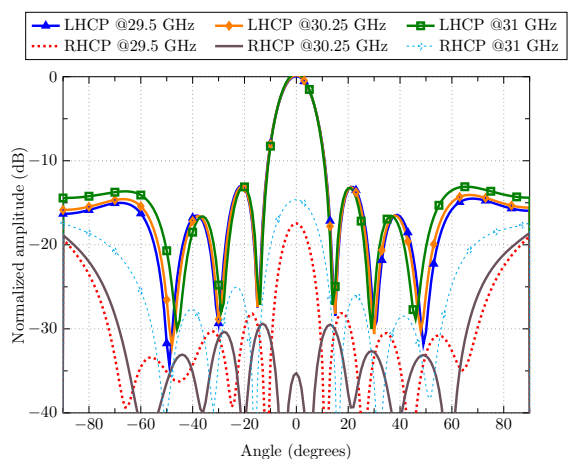

(a)

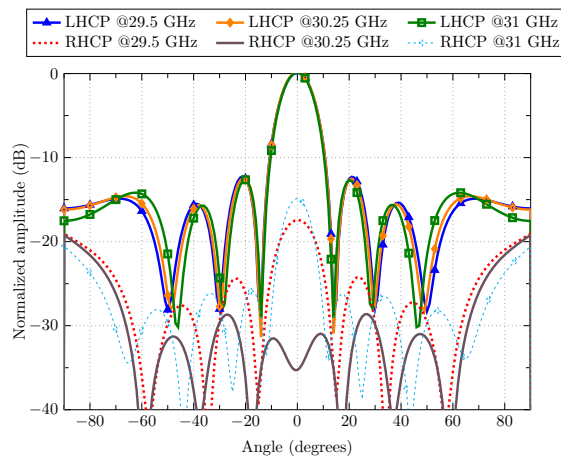

(c)

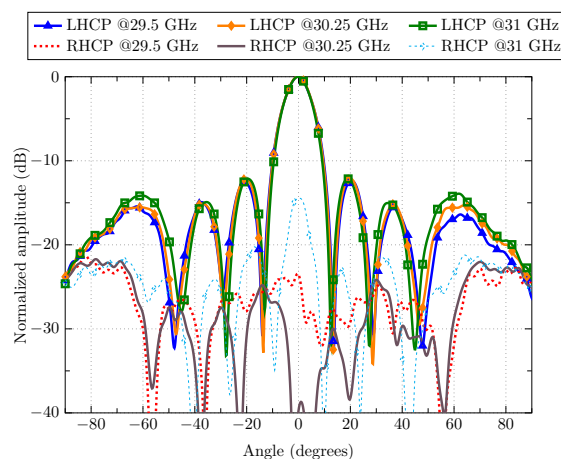

(b)

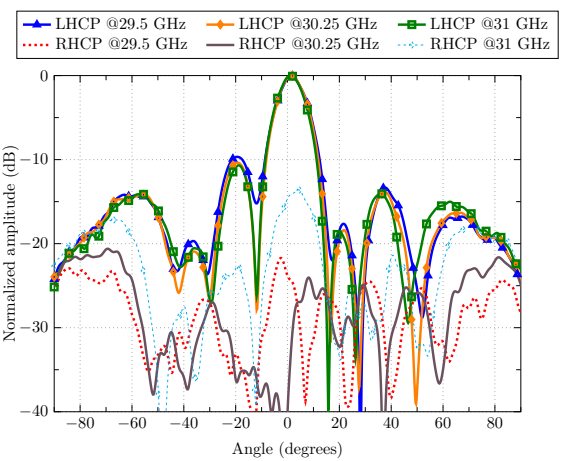

(d)

Figure 4.31: For several frequencies : (a) Simulated E-plane pattern. (b) Measured E-plane pattern. (c) Simulated H-plane pattern. (d) Measured Hplane pattern [235].

Table 4.5: Measured broadside antenna parameters.

\begin{tabular}{|c|c|c|c|c|}
\hline Frequency $(\mathrm{GHz})$ & 29.5 & 30 & 30.5 & 31 \\
\hline \hline Directivity $(\mathrm{dBi})$ & 21.94 & 22.18 & 22.3 & 22.44 \\
\hline Gain $(\mathrm{dBi})$ & 21.83 & 21.95 & 22.25 & 22.40 \\
\hline Antenna Efficiency (\%) & 97.5 & 94.84 & 98.9 & 99.1 \\
\hline
\end{tabular}

\section{Experimental results}

The fabricated prototype was subject to a measurement campaign at several sampled frequencies within the band. For the sake of comparison, simulated 


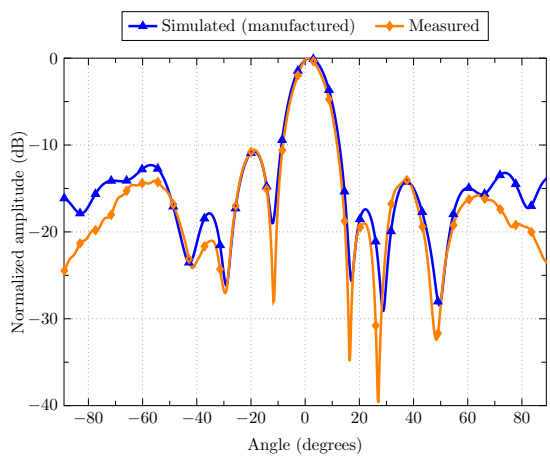

Figure 4.32: Copolar radiation pattern (XZ-plane) at $31 \mathrm{GHz}$ including the machining errors in simulation [235].

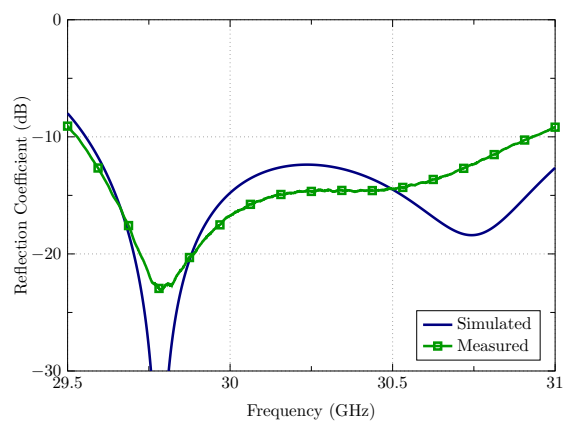

(a)

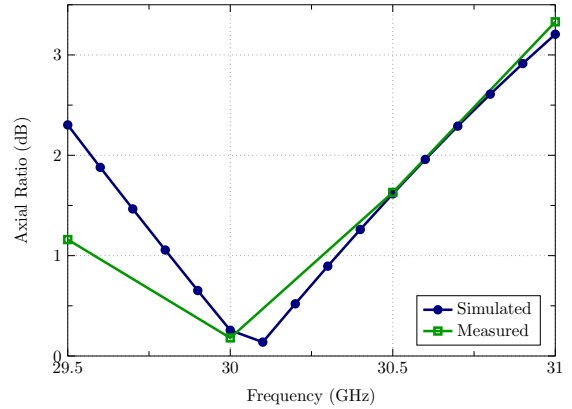

(b)

Figure 4.33: (a) Measured and simulated reflection coefficient versus frequency. (b) Measured and simulated axial ratio versus frequency [235].

copolar and cross-polar radiation patterns are first shown in Figs. 4.31a and 4.31c. These graphs represent $\phi=90^{\circ}$ and $\phi=0^{\circ}$ cuts, respectively. As can be observed, both cuts reveal an excellent stability versus frequency. First sidelobes appear at $-13 \mathrm{~dB}$ level, as can be expected from a uniform illumination. Measured radiation patterns in the main cuts are shown in Figs. 4.31b and 4.31d. Remarkable agreement between simulations and measurements can be noticed along YZ-plane $\left(\phi=90^{\circ}\right)$. XZ-plane, conversely, reveals a clear asymmetry which can be attributed to the manufacturing errors discussed above.

As a further proof of the statement above, the antenna was resimulated including the machining errors detected. Fig. 4.32 shows a much better agreement between measurements and simulations for XZ-plane. Although it is 
difficult to quantify exactly the amount of badly milled metal, this estimation clearly demonstrates the cause behind the XZ-plane pattern asymmetry.

Fig. 4.33 a plots the measured $S_{11}$-parameter for the band of interest. It remains below $-10 \mathrm{~dB}$, as predicted by simulations. With respect to the circular polarization purity, measured and simulated axial ratio are shown in Fig. 4.33b. Nearly ideal $0-\mathrm{dB}$ axial ratio has been sampled at $30 \mathrm{GHz}$, rising up to $1 \mathrm{~dB}$ and $3 \mathrm{~dB}$ for the lower and upper band edges, respectively. Satisfactory agreement with simulations can be noticed, being measurements even better in the lower part of the band. Nevertheless, notice that an axial ratio below $3 \mathrm{~dB}$ might be obtained just by a slight re-tuning toward higher frequencies. Such re-tuning can be done by modifying the angle of the chamfers or the waffle grid height.

Finally, the measured directivity, gain and antenna efficiency are shown in Table 4.5 for four equispaced frequencies at broadside direction. Measured efficiency values, over 94\%, are particularly remarkable for this $30 \mathrm{GHz}$ targeted band. Such good results are consequence of the full-metallic waveguiding technology and the gap-waveguides capability to prevent field leakage. Notice, however, that directivity figures are obtained from a numerical integration of the measured full radiation pattern. Therefore, it is affected by the presence of the positioner and subjected to certain uncertainty in the tenths of $\mathrm{dB}$. Obviously, antenna efficiency is directly affected by this uncertainty. Also, it must be recalled that, as any corporate-fed array, efficiency values will decrease as aperture size becomes larger. Gain measurements has been carried out at the anechoic chamber facilities of Universidad Politécnica de Madrid (UPM), Spain, which are ISO-17025 standard certified. The uncertainty in measured gain values for an antenna of this size in Ka-band is around $\pm 0.18 \mathrm{~dB}$. Therefore, even in the worst case, the measured efficiency values would be above $90 \%$.

\section{Conclusions}

Combined GGW-RGW network has allowed us to feed uniformly and in phase not only the coaxial cavities but also the cylindrical cavities. Truncated circular waveguides allow, in a single layer, to feed circularly-polarized radiators. The compact architecture avoids using a multilayer array. Also, its structure resembles to microstrip arrays but completely metallic, which leads to a highefficiency array in the Ka band (above $90 \%$ ). The proposed array architecture may be seamlessly enlarged to any size thanks to the scalability of the gap-based corporate feeding network, making this solution very attractive for medium to high-gain applications. 


\section{4 $8 \times 8$ Dual-Polarized Array Antenna}

The three prototypes seen so far are based on single-layer solutions. The next antennas presented are multilayer structures but they offer additional features such as dual-polarization or dual-band.
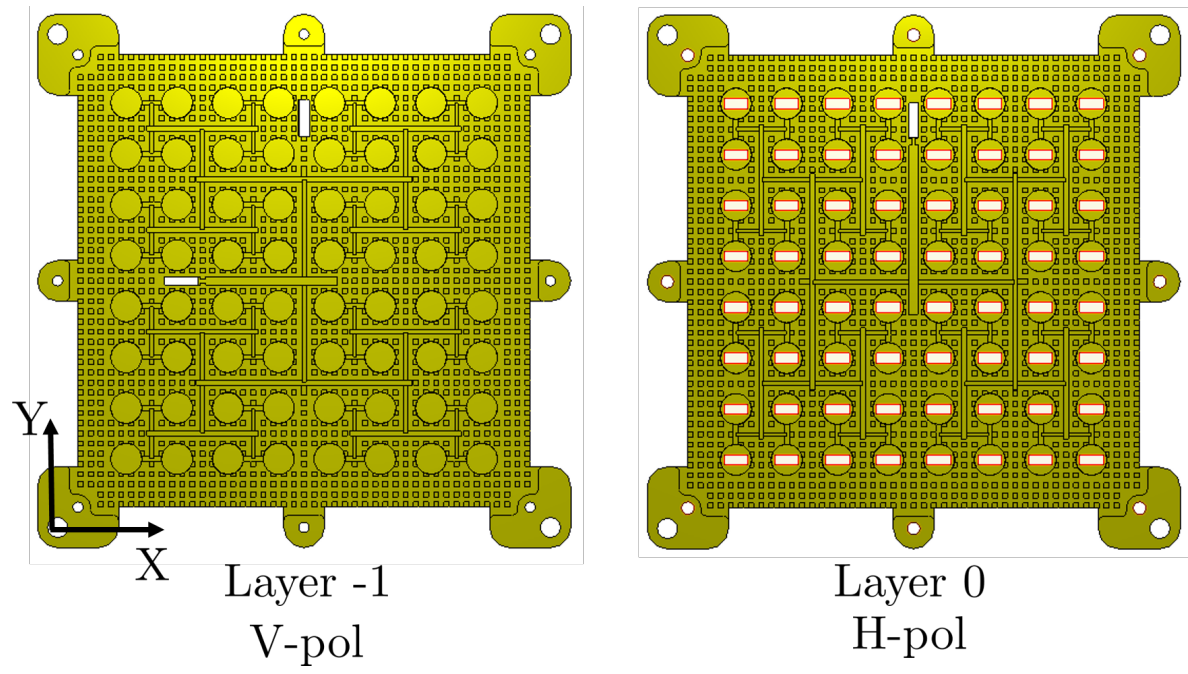

Figure 4.34: Top view of layers -1 and 0 of the antenna.

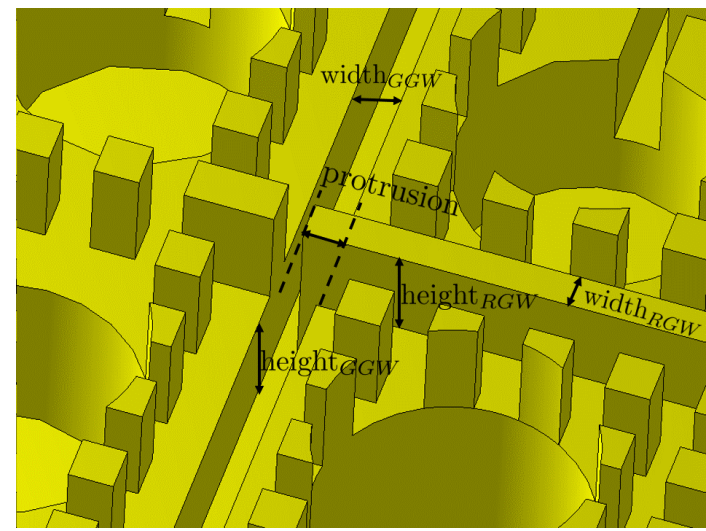

Figure 4.35: Combined RGW-GGW divider. The fundamental parameters of each waveguide are indicated. 


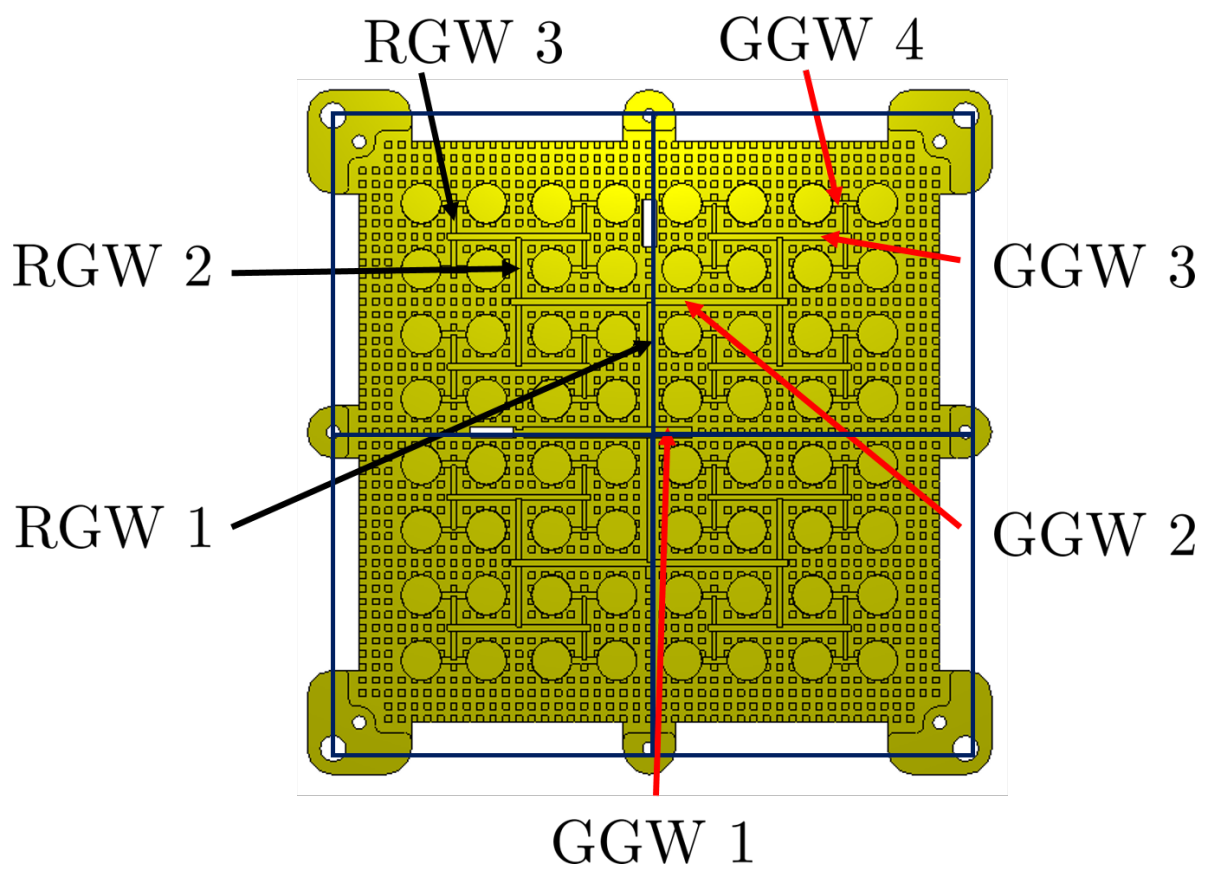

Figure 4.36: The total network is composed of 4 GGW and 3 RGW of different dimensions. By symmetry the network is identical in each quadrant.

Table 4.6: Relevant dimensions of the distribution network in both layers.

\begin{tabular}{|l|c|c|c|}
\hline & Width $\mathbf{( m m})$ & Height $(\mathbf{m m})$ & \multirow{2}{*}{} \\
\cline { 2 - 3 } Groove 1 & 1.75 & 2.61 & \\
Groove 2 & 1 & 2.57 & \\
\cline { 2 - 3 } Groove 3 & 1 & 2.57 & \\
\cline { 2 - 3 } Groove 4 & 1 & 2.67 & 0.37 \\
\hline & Width $\mathbf{( m m )}$ & Height $(\mathbf{m m})$ & Protrusion $\mathbf{( m m )}$ \\
\hline Ridge 1 & 1 & 2.3 & 0.36 \\
\hline Ridge 2 & 0.78 & 1.5 & 0.4 \\
\hline
\end{tabular}

The first multilayer prototype is a dual-polarized antenna that uses stacked cylindrical cavities as radiating element. These cylindrical cavities are excited in such a way that in the top layer the two orthogonal components of the field (horizontal and vertical linear polarization) are radiated. A preliminary 
approach at this antenna was the result of a collaboration with the Chalmers group [182]. Here we will concentrate on an evolved version of it.

Fig. 4.34 shows each of the layers that compose the antenna in the absence of the top lid. The periodicity of nails, height and width are exactly the same for each layer. The diameter of the apertures as well as the dimensions of the corporate-feed distribution network are similar too.

Fig. 4.35 shows one of the RGW-GGW dividers of the network with the legend providing the most relevant parameters. Fig. 4.36 illustrates that the full antenna can be divided into four identical quadrants (blue squares of the figure). The arrows indicate that three different RGW and four GGW sets can be found in the whole 1-to-64 corporate network. Waveguide dimensions are indicated in Table 4.6.

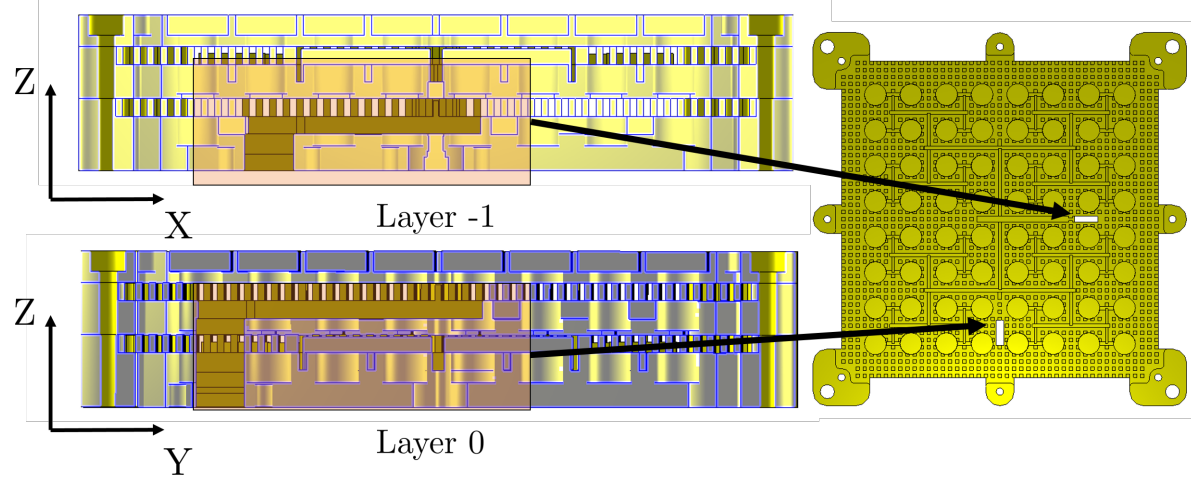

Figure 4.37: Side view of the transitions and top view of the antenna where their location on it is indicated.

\section{Input Ports}

There are just a few differences between both layers but these are key. In the lower layer (Layer -1), the floor of the cylindrical cavities is solid conductor. However, in the intermediate layer (Layer 0), the floor contains a slot. This slot is responsible for coupling both layers. The slot is placed perpendicular to the field of Layer 0, so there is no coupling to Layer -1 . Another difference is the input port of each layer. The input port to the lower layer is directly connected from the back of the antenna while the input port of the intermediate layer must cross Layer -1 , as illustrated in Fig. 4.37. A good realization of this transition will be key to obtaining good matching.

In both cases the transitions have been designed following the same strategy. A standard WR-28 input waveguide - whose dimensions are $7.112 \mathrm{~mm} \times 3.556 \mathrm{~mm}$ 


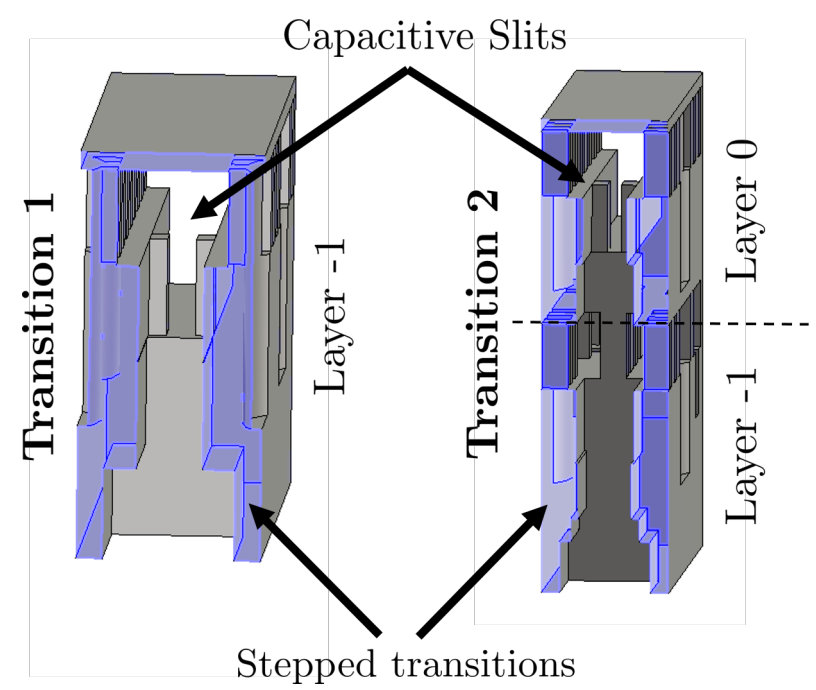

Figure 4.38: Side view showing the stepped transition and the capacitive windows at the GGW input.

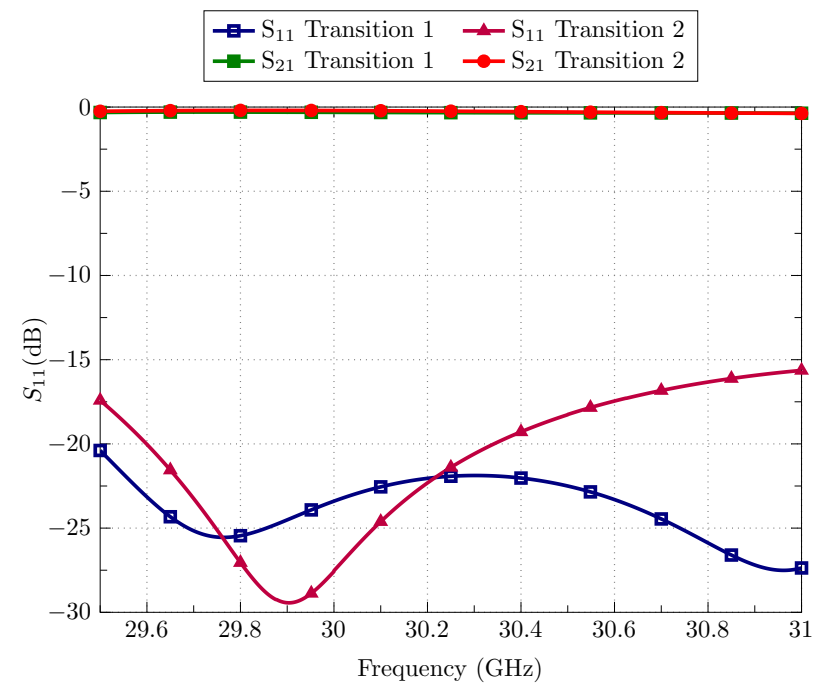

Figure 4.39: Scattering parameters of the two input transitions.

- must be adapted with a series of steps to the width of the GGW, which in both cases is $1.75 \mathrm{~mm}$, as shown in the Table 4.6 . 


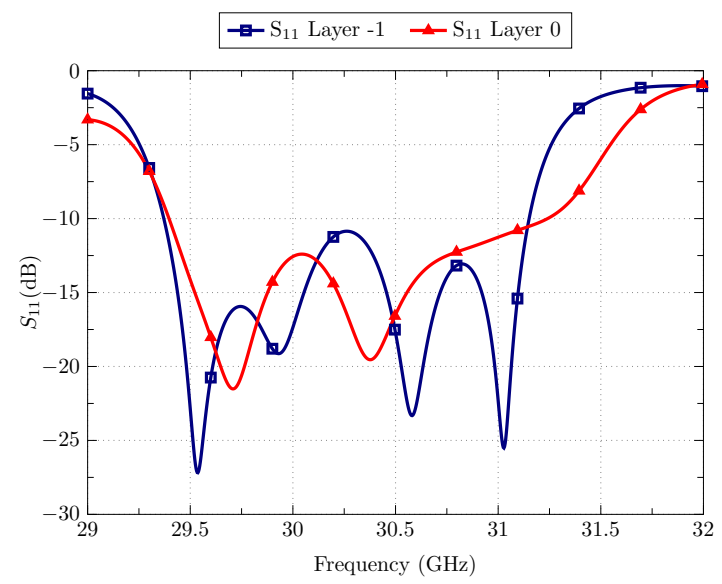

Figure 4.40: Simulated reflection coefficients of the Dual-Polarized Antenna.

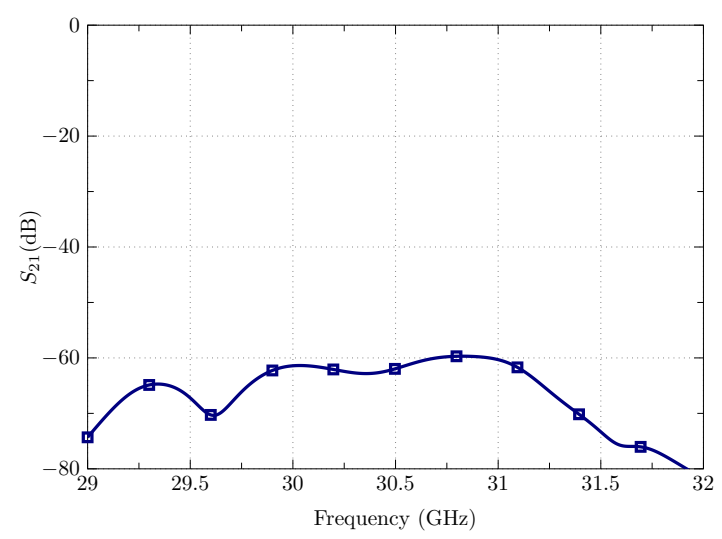

Figure 4.41: Simulated $\mathrm{S}_{21}$ parameters of the Dual-Polarized Antenna.

It should be noted that this groove is $75 \%$ wider than the rest of the GGW's of the antenna precisely to favor the matching from the WR-28 to the input port. The capacitive slits at the GGW input are as relevant as the steps. These capacitive windows can be clearly seen in Fig. 4.38. Both inputs have these slits. Scattering parameters of the two input transitions were optimized separately and are shown in Fig. 4.39 . 


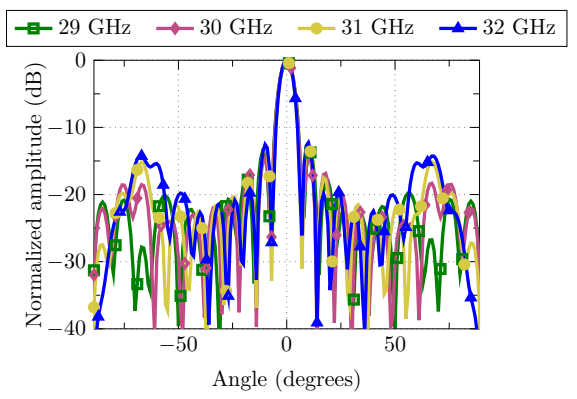

(a)

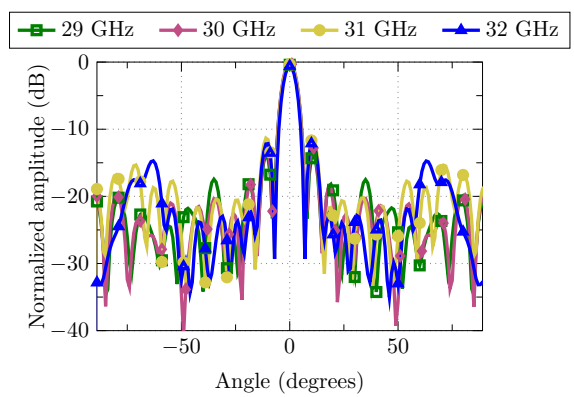

(c)

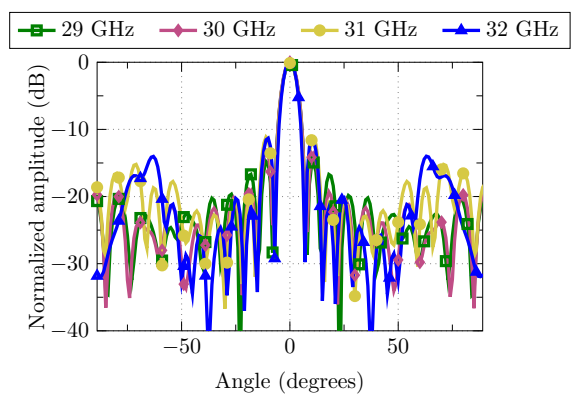

(b)

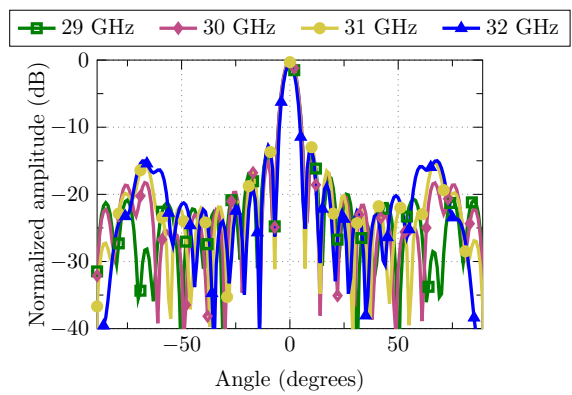

(d)

Figure 4.42: For several frequencies : (a) Simulated XZ-plane pattern (Layer -1). (b) Simulated YZ-plane pattern (Layer -1). (c) Simulated XZ-plane pattern (Layer 0).(d) Simulated YZ-plane pattern (Layer 0).

\section{Simulated Results}

Any two-port antenna has the added challenge of having to provide good isolation between them. Fig. 4.40 shows that both $\mathrm{S}_{11}$-parameters are below the target of $-10 \mathrm{~dB}$ from $29.5 \mathrm{GHz}$ to $31 \mathrm{GHz}$ which is a usual band for transmit in the SOTM applications in the Ka band. Fig. 4.41 shows the isolation between ports, with a decoupling of more than $60 \mathrm{~dB}$ in the entire bandwidth. Regarding the radiation patterns in Fig. 4.42, as it would be expected from a dual-polarized antenna, the XZ-plane cut of polarization 1 is exactly the same as the YZ-plane cut of polarization 2 and analogously in the other cuts. Finally, Fig. 4.43 shows the crosspolar component of the vertically-polarized component, being less than $-60 \mathrm{~dB}$ in the whole band. The same results are obtained for the horizontal polarization. 


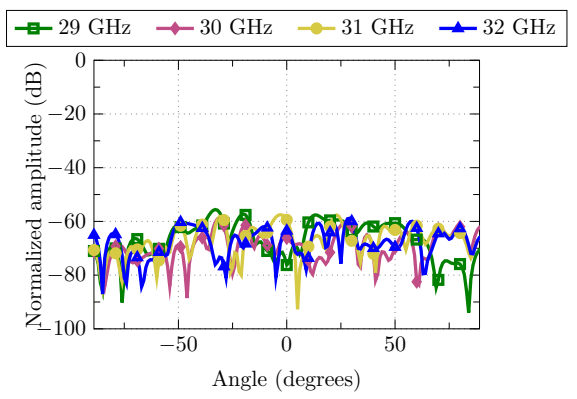

(a)

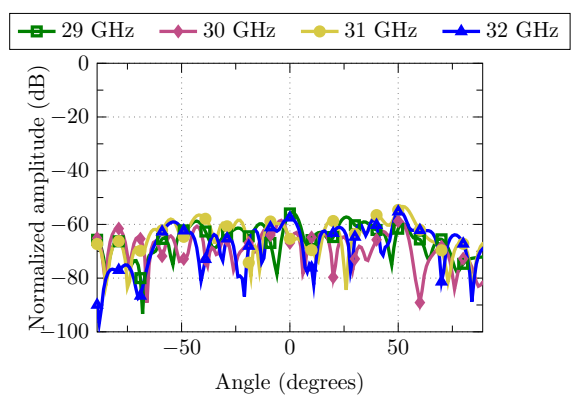

(b)

Figure 4.43: (a) Crosspolar of XZ-plane for V-pol (Layer -1). (b) Crosspolar of YZ-plane for V-pol (Layer -1).

\section{Manufacturing process}

This time the manufacturer of the prototype was the Chinese company NextProto. A lower cost option was attempted to check the performance that can be expected. Fig. 4.44 shows pictures of the fabricated antenna. Looking at the antenna in detail using a microscope, it was observed that sensitive manufacturing parts such as the distribution network, transitions or input ports were far from an ideal fabrication. These serious manufacturing errors led to a very poor measured results.

Fig. 4.45 illustrates in the images of the left column two critical parts which are key for good antenna functioning. In Figs. 4.45a and 4.45b, a power divider from RGW to GGW is shown and in Figs. $4.45 \mathrm{c}$ and $4.45 \mathrm{~d}$ a transition from GGW to RGW is detailed. The figures on the right side correspond to two dividers at the same frequency manufactured later following the agreed tolerances. Comparing Figs. $4.45 \mathrm{a}$ and $4.45 \mathrm{~b}$ it is clear that in the first case there is no straight continuity in the GGW, making the performance of the transition worse since the fields are not well coupled from the RGW to the GGW. This inconsistency is more noticeable in Figs. $4.45 \mathrm{c}$ and $4.45 \mathrm{~d}$. Whereas Fig. $4.45 \mathrm{~d}$ clearly shows a groove with uniform and straight walls, Fig. $4.45 \mathrm{c}$ is a deformed groove with asymmetric walls and rounded inner corners showing less than acceptable tolerance (of the order of $0.2 \mathrm{~mm}$ ).

This study has allowed us to identify the most sensitive parts in the GW manufacture and to what extent bad manufacturing results in inconsistent and unacceptable measurements. The tolerances required in GW technology are not at all limiting and even less at $30 \mathrm{GHz}$ but poor manufacturing without taking due care can cause the errors shown here. 


\section{CHAPTER 4. GAP WAVEGUIDE ARRAY ANTENNAS}
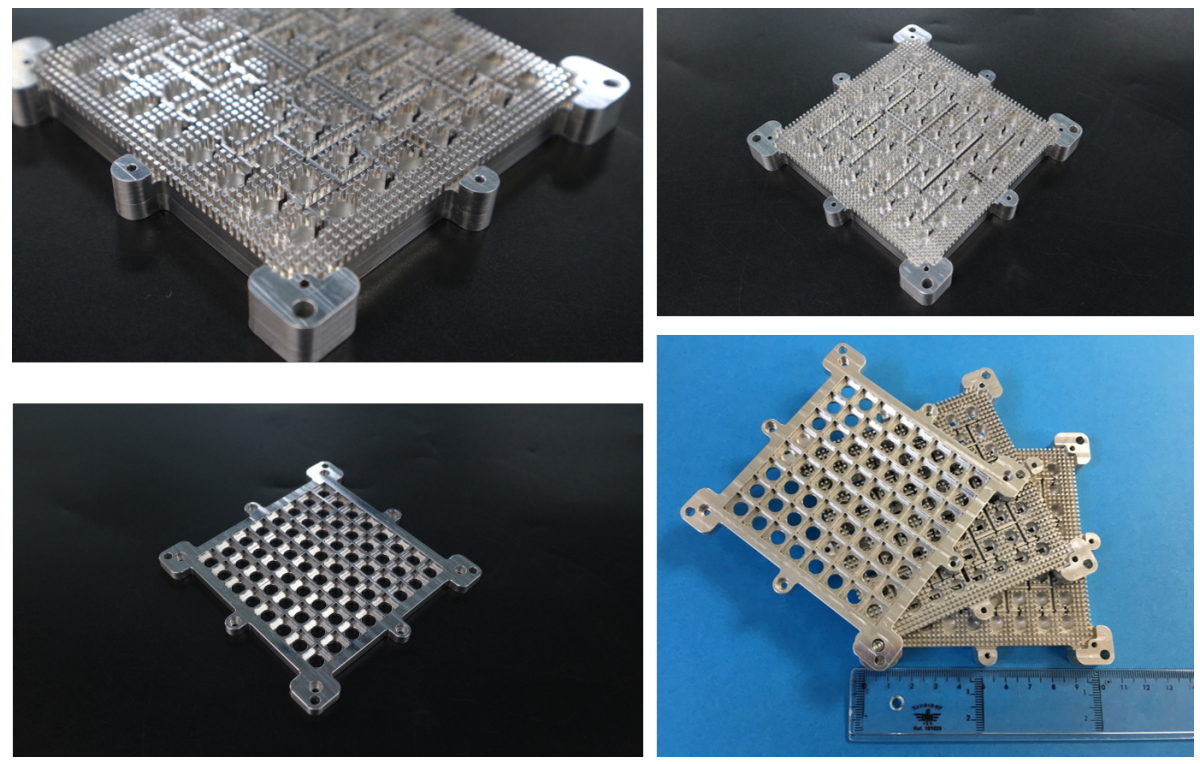

Figure 4.44: Photographs in detail of the fabricated dual-polarized antenna.

A second antenna was manufactured at iTEAM-UPV facilities. The new model comply with the expected tolerances so that we could compare our manufacturing capacity with that of NextProto. Fig. 4.46 shows the antenna in the manufacturing process in the iTEAM-UPV facilites.

\section{Experimental results}

The experimental validation of the manufactured prototype is observed in Figs. 4.47 and 4.48. The first of these figures corresponds to the reflection coefficient measured for each of the ports. The left figure corresponds to port 1 (which feeds layer -1 ) and the right figure to port 2 (which feeds Layer 0). In these figures a comparison is made between simulation and measurement. Although the agreement is not perfect, it maintains a similar behavior.

These measurements have a reflection coefficient below $-10 \mathrm{~dB}$ from 29.5 to $30.8 \mathrm{GHz}$ (close to the goal of covering a 1.5-GHz bandwidth). As for port 2, best matching is achieved from $29.8 \mathrm{GHz}$ to $31.2 \mathrm{GHz}$. Overlapping both results and taking the most restrictive results, the antenna presents an adaptation of $1 \mathrm{GHz}(29.8$ to $30.8 \mathrm{GHz})$ below $-10 \mathrm{~dB}$.

Regarding the radiation patterns, the main cuts are presented at various frequencies (from $29.5 \mathrm{GHz}$ to $31 \mathrm{GHz}$ ) and with both polarizations (horizontal and vertical). The results are highly satisfactory. Uniform patterns with 


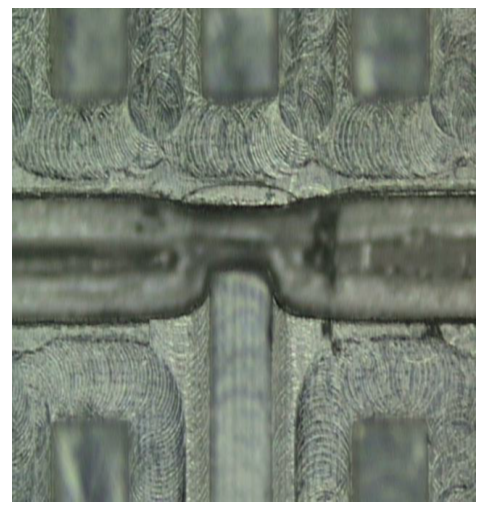

(a)

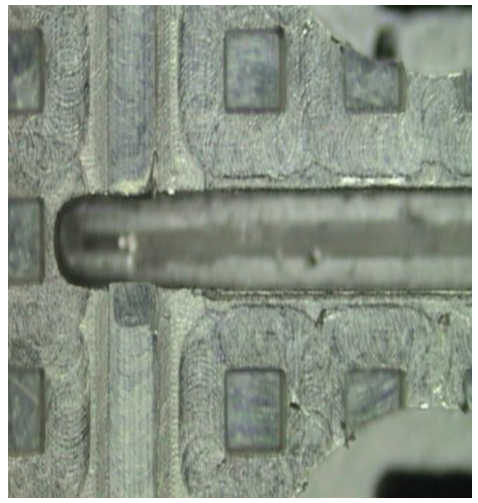

(c)

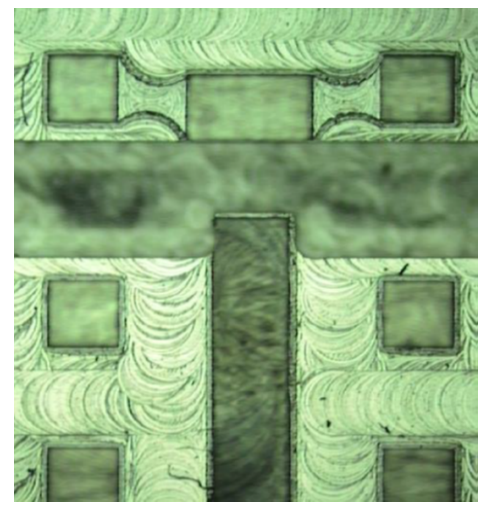

(b)

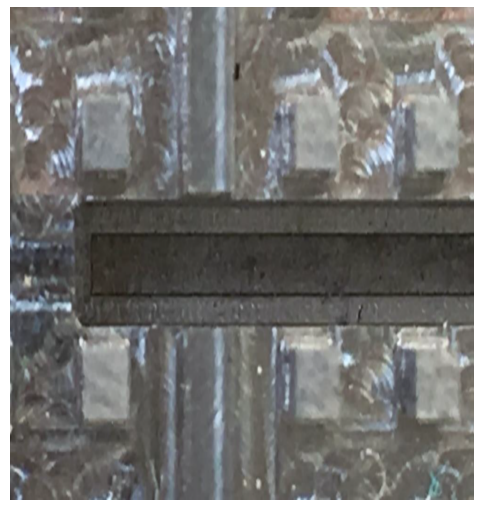

(d)

Figure 4.45: Pictures of the metrological study to validate the manufacturing accuracy. (a) and (c) correspond to NextProto manufacturing. (b) and (d) to UPV manufacturing.

well-defined lobes, very similar to those obtained in simulation. This antenna has a directivity of $27 \mathrm{dBi}$ and an antenna efficiency better than $75 \%$ over the entire bandwidth.

\section{Conclusion}

Dual-polarized array antenna has been an evolution that complements the single-layer circularly-polarized array previously described. The circularly- 


\section{CHAPTER 4. GAP WAVEGUIDE ARRAY ANTENNAS}

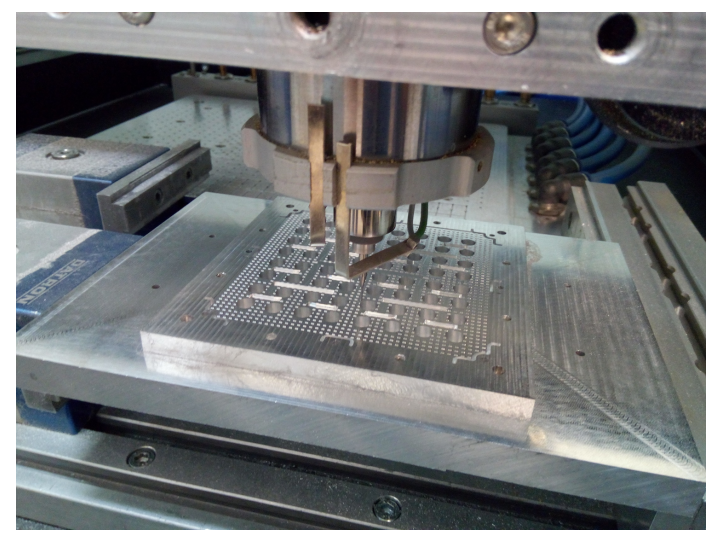

Figure 4.46: Antenna during the manufacturing by milling.

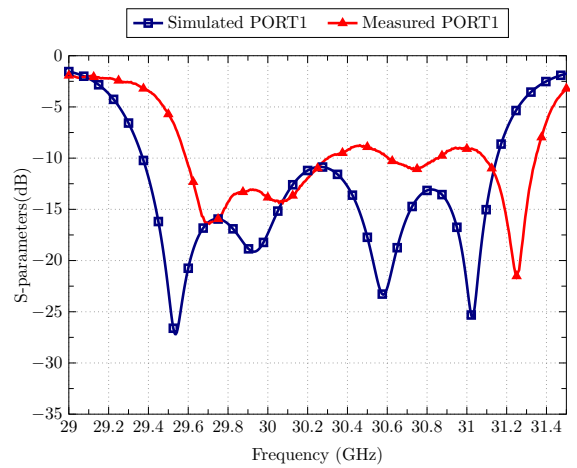

(a)

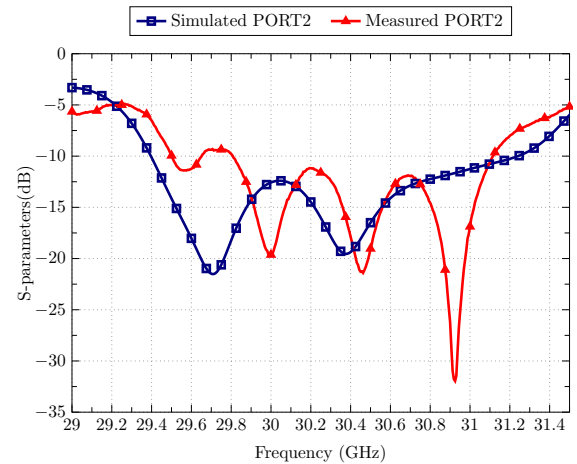

(b)

Figure 4.47: Measured and simulated $\mathrm{S}_{11}$ parameters in both ports.

polarized array presented earlier is unable to provide both polarizations. Therefore, a trade-off has been reached adding an intermediate layer to provide both horizontal and vertical polarization. Now, by feeding the ports conveniently, it is possible to radiate both orthogonal linear components or RHCP and LHCP. The integration of a hybrid coupler in the back side of the antenna remains open as a future development. 


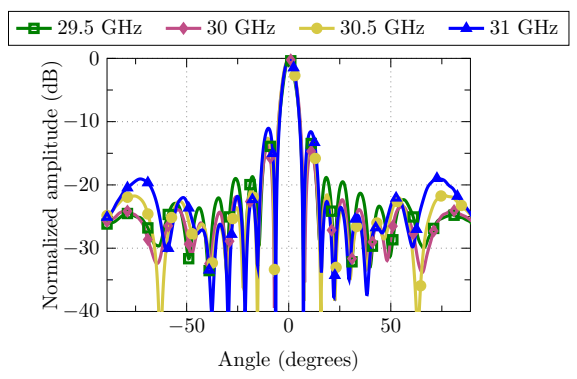

(a)

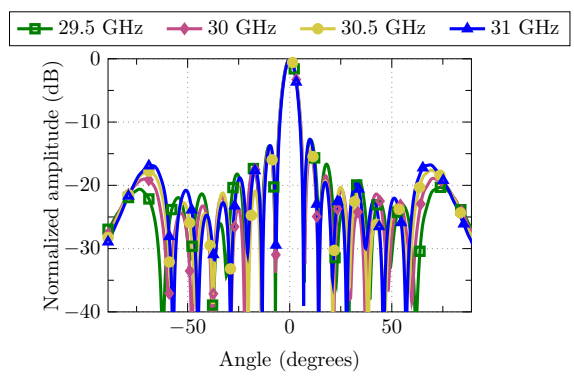

(c)

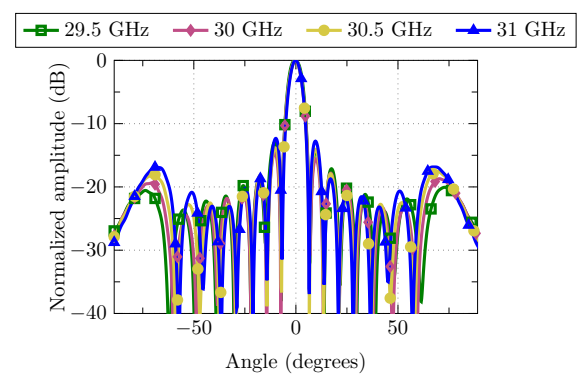

(b)

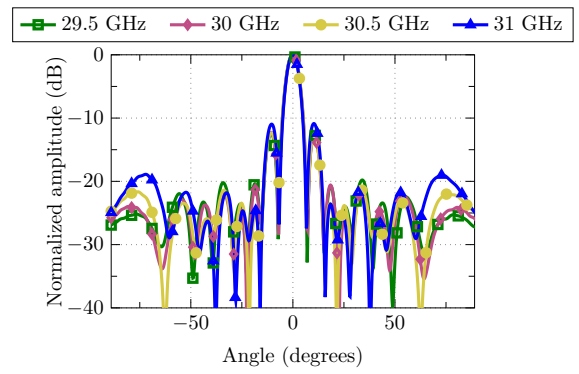

(d)

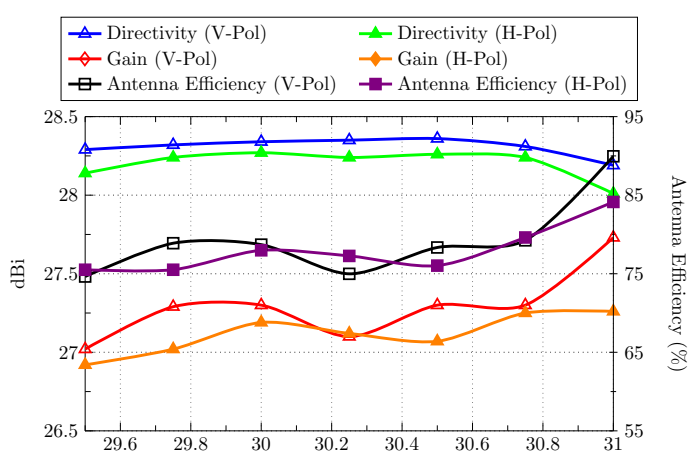

(e)

Figure 4.48: For several frequencies : (a) Measured XZ-plane pattern (Layer 1). (b) Measured YZ-plane pattern (Layer -1). (c) Measured XZ-plane pattern (Layer 0).(d) Measured YZ-plane pattern (Layer 0). (e) Measured directivity and gain. 


\section{5 $8 \times 8$ Single-Layer Dual-Band Cavity-Backed Slot Array Antenna}

The following architecture propose the use of coaxial cavities to integrate two antennas for widely separated frequency bands within the same panel. As seen in Chapter 3, adjusting the height of these cavities it is possible to have resonators at different frequencies. Now, since the objective is to provide new solutions for SATCOM applications, bands 19.7-21.2 GHz for receive and 29.5$31 \mathrm{GHz}$ for transmit have been assumed.

\section{Discussion on the relative position of the radiating elements within the panel}

Once the versatility to create coaxial cavities that resonate at different frequencies has been proven, the question is how to place these cavities in a single layer. In order to obtain a radiation pattern without grating lobes a limiting factor is the spacing between apertures.

Spacing larger than $\lambda_{0}$ will lead to the appearance of the grating lobes. If the elements are evenly distributed (same number of TX and RX apertures), TX radiation pattern will have higher SLL than the RX one. A first guess to solve this issue is to place more TX apertures than RX ones, but even this solution is not as simple because what is improved for the TX pattern will worsen the RX pattern by having fewer elements. All these circumstances make the arrangement of the elements in the panel a key decision.

It was decided to make an analytic study of the best possible solution, assessing the pros and cons of each of the possible options:

- A 2 to 1 vertical ratio. That is 2 times more TX apertures than RX in an horizontal arrangement.

- A 2 to 1 ratio in horizontal. Same as the previous case but in a vertical arrangement.

- A grid layout, where there is the same number of RX as TX apertures, alternately arranged.

Arrangement 1 proposes to place two TX elements for each RX one in the vertical dimension to obtain radiation patterns with the lowest possible SLL at both frequencies ( $\mathrm{RX}$ and TX). It must be emphasized that the analytic study only considers isotropic sources, therefore the Array Factor (AF) is not multiplied by any element pattern.

In Fig. 4.49 TX slots are marked with red crosses. RX slots are drawn as blue circles. The distance between elements is the minimum distance allowed by 


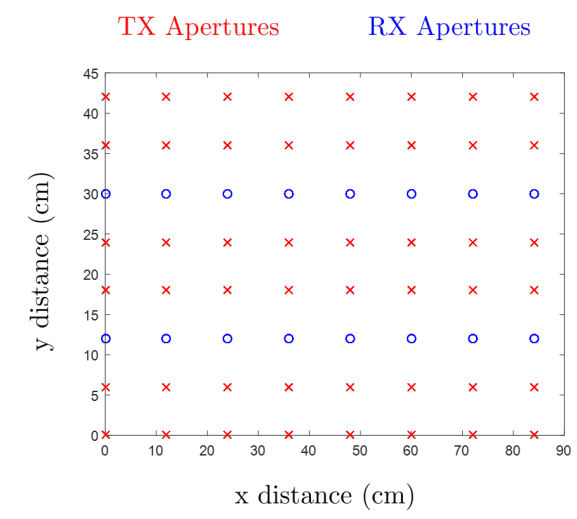

Figure 4.49: Arrangement 1. Two rows of TX radiating elements are placed for each row of RX.

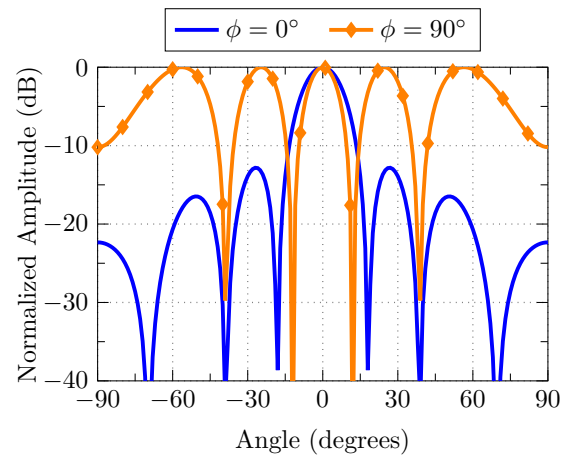

(a)

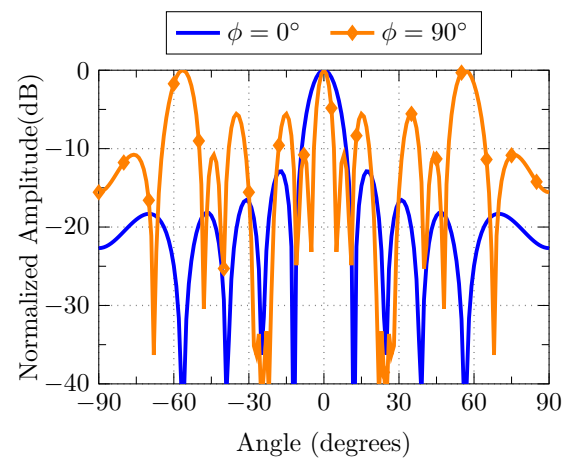

(b)

Figure 4.50: Simulated AF using the arrangement 1: (a) $20 \mathrm{GHz}$ and (b) 30 $\mathrm{GHz}$.

the RGW-GGW distribution network. On the X-axis, the minimum distance at which the elements can be placed is $12 \mathrm{~mm}$ while on the $\mathrm{Y}$-axis the minimum distance is $6 \mathrm{~mm}$. On the $\mathrm{Y}$-axis it is much easier to place coaxial cavities because they are placed on both sides of the GGW. In the X-axis, the elements must be more separated since the power divider is in-between them.

Fig. 4.50a represents the AF for RX elements (blue circles) in the two main cuts $\left(\phi=0^{\circ}\right.$ and $\left.\phi=90^{\circ}\right)$. XZ-plane shows good appearance since the elements are spaced $12 \mathrm{~mm}$, which is $0.8 \lambda_{0}$ at $20 \mathrm{GHz}$. For YZ-plane, the spacing is $24 \mathrm{~mm}$, which is $1.6 \lambda_{0}$, and grating lobes show up. For the TX pattern in 


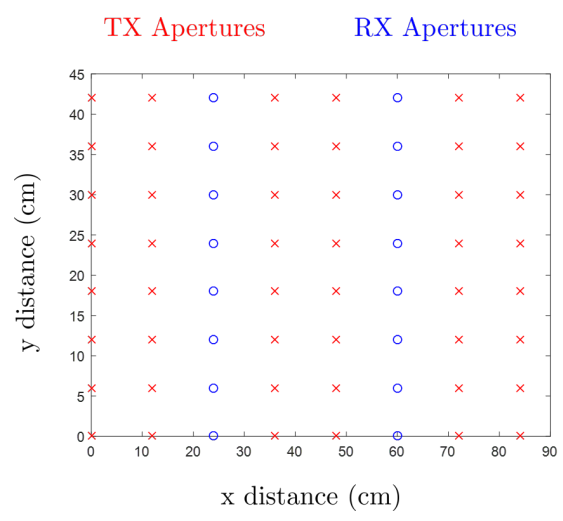

Figure 4.51: Arrangement 2. Two columns of TX radiating elements are placed for each column of RX.

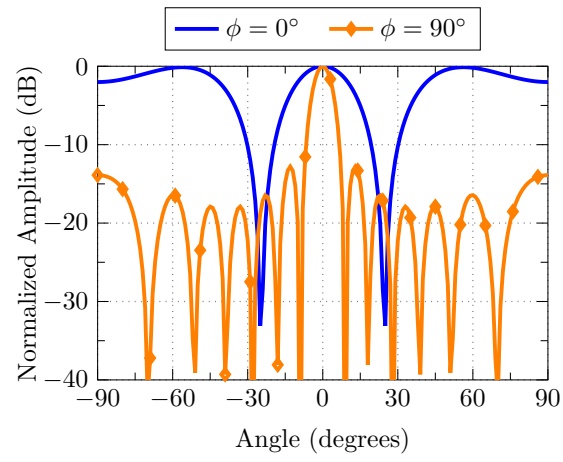

(a)

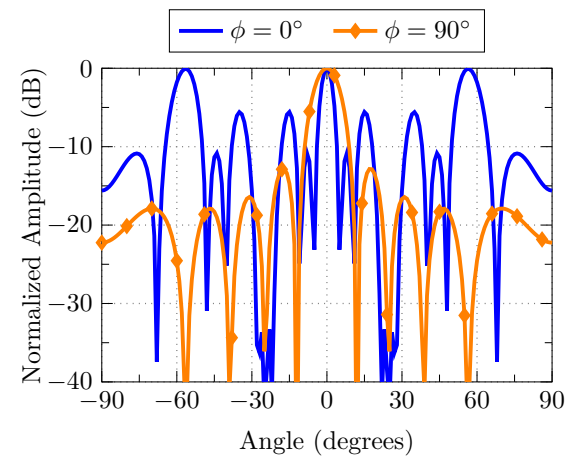

(b)

Figure 4.52: Simulated AF using the arrangement 2: (a) $20 \mathrm{GHz}$ and (b) 30 $\mathrm{GHz}$.

Fig. $4.50 \mathrm{~b}$, the $\phi=0^{\circ}$ cut remains fairly stable, but the $\phi=90^{\circ}$ cut exhibits grating lobes at $\theta=60^{\circ}$.

In the second arrangement, a 2 to 1 ratio in the horizontal dimension is used. See Fig. 4.51. As expected, the situation is similar with regard to the grating lobes but in the orthogonal cut now, as seen in Fig. 4.52.

A first conclusion that can be drawn is that an entire column or row of elements of a given frequency results in a shadow area for the other frequency that it is too large. This leads to degradation of the radiation pattern. For these reason, a trade-off must be reached. Whatever the element distribution, 


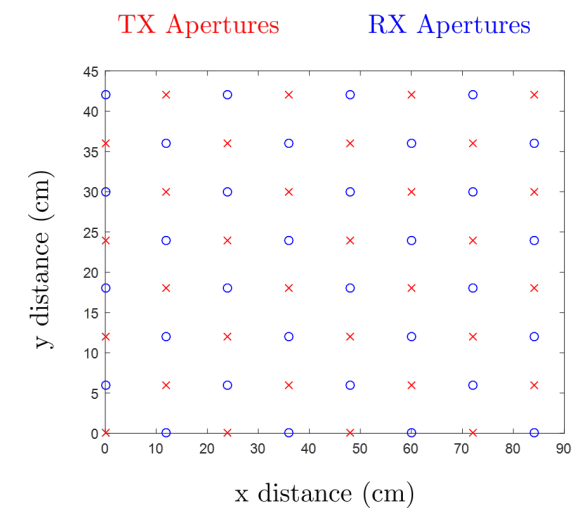

Figure 4.53: Arrangement 3. Elements are interleaved.

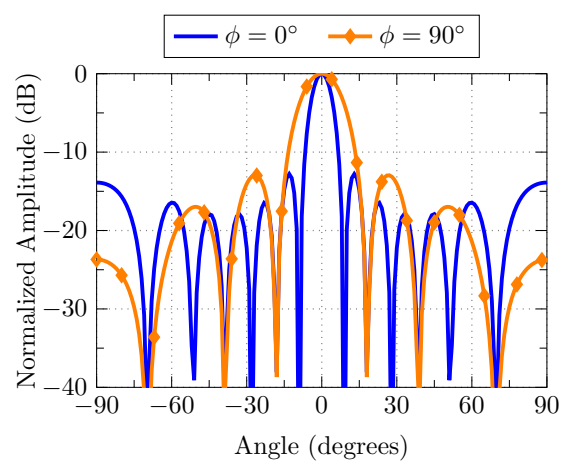

(a)

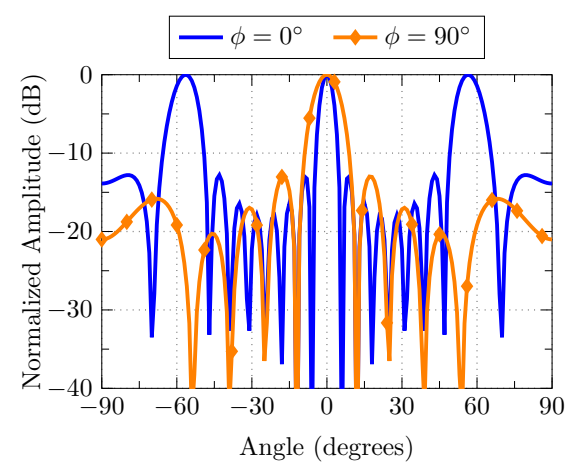

(b)

Figure 4.54: Simulated AF using the arrangement 3: (a) $20 \mathrm{GHz}$ and (b) 30 $\mathrm{GHz}$.

keeping two well-separated bands in a single panel seems difficult to achieve. However, the solution could be to be able to get at least 3 of the 4 main cuts with acceptable SLLs. Ultimately the grating lobes in the fourth cut could be mitigated using ad-hoc superstrates or sets of longitudinal grooves between elements, as it will be explained later.

The third arrangement (Fig. 4.53) corresponds to a lattice where both types of slots are interleaved. Fig 4.54a shows a uniform AF in both cuts at $20 \mathrm{GHz}$. In the Fig. 4.54b, which corresponds to the TX AF, grating lobes only appear for $\phi=0^{\circ}$ cut. Therefore, even if it is not the ideal scenario yet, it can be a good starting pont to the desired single-layer dual-band array. 


\section{CHAPTER 4. GAP WAVEGUIDE ARRAY ANTENNAS}
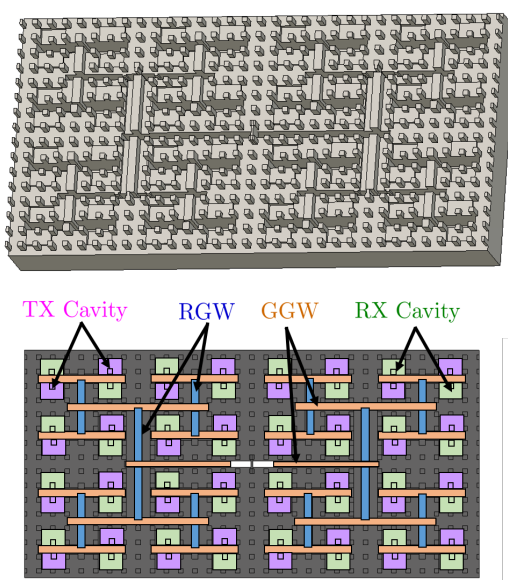

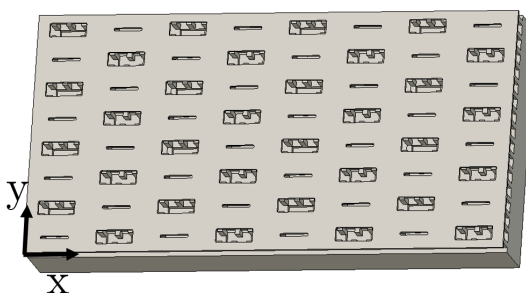

RX Apertures

TX Apertures

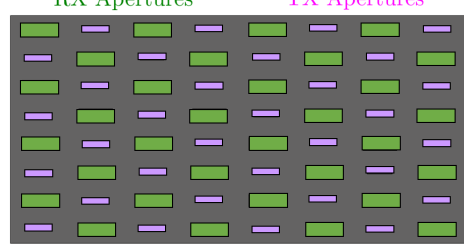

Figure 4.55: Array perspective and frontal views (with and without cover) where the parts that make up the distribution network, the location of the coaxial cavities and each type of aperture have been coloured for clarity.

\section{Antenna Geometry}

This section describes the design and dimensions of the arrangement chosen after the previous discussion. The antenna consists of 64 elements distributed in 8 rows by 8 columns. Of the 64 elements, 32 are TX and 32 are RX, being interleaved in the panel.

The $8 \times 8$ antenna array is illustrated in Fig. 4.55. The upper part of this figure shows the CST model of the array. The location and spacing of the elements have been kept as in the discussion above, $12 \mathrm{~mm}$ in the $\mathrm{X}$-axis and $6 \mathrm{~mm}$ in the Y-axis. In the lower part, the elements of the array are sketched and the most relevant parts of the antenna are indicated. First of all, the full-corporate feed network is observed. GGW sections are depicted in orange while RGW sections of the network are coloured in blue. It should be noted that each end of the network feede two coaxial cavities, one for each frequency, RX and TX. Therefore the 1 to 32 network can feed 64 elements in a corporate way, symmetrically and uniformly.

In tables (4.7 and 4.8) all array dimensions are shown. Firstly, the dimensions of the nails as well as their periodicity are indicated. The dimensions of the respective GGW and RGW are also shown. As in the case of the dualpolarized antenna, the waveguides are identical by symmetry and there are only three GGW and two RGW with different dimensions. The numbering 
Table 4.7: Design parameters of the Dual-band Array (Feeding Layer)

\begin{tabular}{|c|c|c|}
\hline \multicolumn{3}{|c|}{ Feeding Layer. } \\
\hline Component & Parameter & Value $\mathbf{( m m}$ ) \\
\hline \multirow{2}{*}{ Nails } & $w \times h$ & $1 \times 3$ \\
& Period & 3 \\
\hline RGW (1) & & $2.4 \times 1.6$ \\
\hline RGW (2) & \multirow{4}{*}{$(h \times w)$} & $2 \times 1.6$ \\
\hline GGW (1) & & $9.1 \times 1.2$ \\
\hline GGW (2) & & $9.1 \times 1.1$ \\
\hline GGW (3) & \multirow{3}{*}{$h e i g h t$} & 1.4 \\
\hline TX Nail & & 2.2 \\
\hline RX Nail & & 0.57 \\
\hline TX Cavity & & -1 \\
\hline RX Cavity & &
\end{tabular}

Table 4.8: Design parameters of the Dual-band Array (Radiating Layer).

\begin{tabular}{|c|c|c|}
\hline \multicolumn{3}{|c|}{ Radiating Layer } \\
\hline Component & Parameter & Value $(\mathbf{m m})$ \\
\hline \multirow{4}{*}{ RX Apertures } & Length & 7.4 \\
\cline { 2 - 3 } & Width & 2.8 \\
\cline { 2 - 3 } & Spacing along $X$ & 12 \\
\cline { 2 - 3 } TX Apacing along $Y$ & 6 \\
\hline \multirow{4}{*}{ Apertures } & Length & 5.6 \\
\cline { 2 - 3 } & Width & 0.6 \\
\cline { 2 - 3 } & Spacing along $X$ & 12 \\
\cline { 2 - 3 } & Spacing along $Y$ & 6 \\
\hline
\end{tabular}

starts from the input port located in the middle of the array. The height of the coaxial nails is shown too. Finally, the cavities are either on a pedestal, TX cavities, or slightly sunk, RX cavities. The indicated dimension is the displacement with respect to the floor of the bed of nails. For that reason, RX cavity height shows a negative value since it is deeper than the floor of the bed of nails.

\section{Preliminary results}

Preliminary simulated results of the antenna are presented here. The reflection coefficient of the array is shown in Fig. 4.56. The two working bands are clearly 
marked. In the RX band the antenna is well matched from $19.5 \mathrm{GHz}$ to 21 $\mathrm{GHz}$ while the TX band covers from $29.5 \mathrm{GHz}$ to $31 \mathrm{GHz}$.

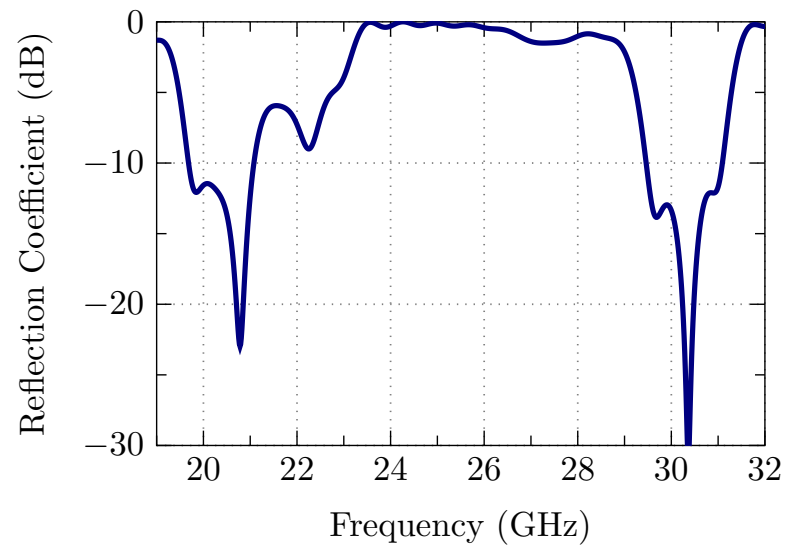

Figure 4.56: Simulated reflection coefficient of the dual-band array.

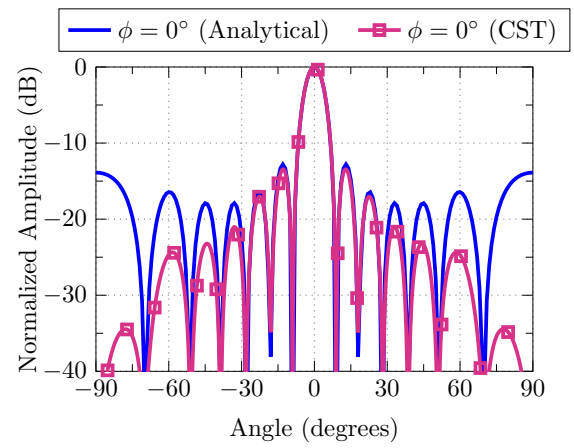

(a)

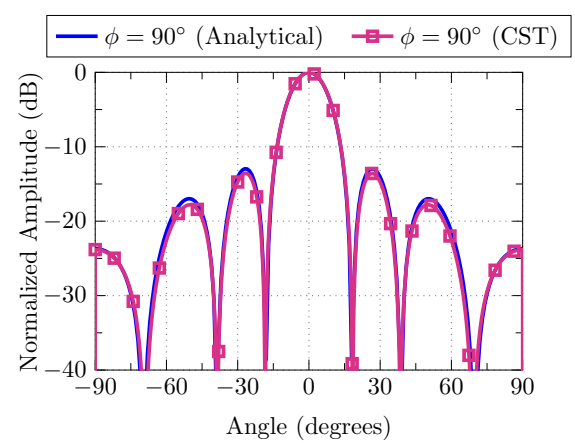

(b)

Figure 4.57: Comparison at $20 \mathrm{GHz}$ of the Array Factor calculated analytically and radiation patterns simulated in CST. (Left) XZ-plane, (Right) YZ-plane.

As for the radiation patterns, it is now possible to compare the agreement between the previous analysis and the complete electromagnetic simulation. Fig. 4.57 compares the RX simulated patterns with the previous AFs. H-plane is identical since the slot radiates omnidirectionally in this cut. On the other hand, E-plane patterns are not equal due to the effect of the slot pattern, left subfigure. The radiation null of the slot favors the progressive decrease of the 


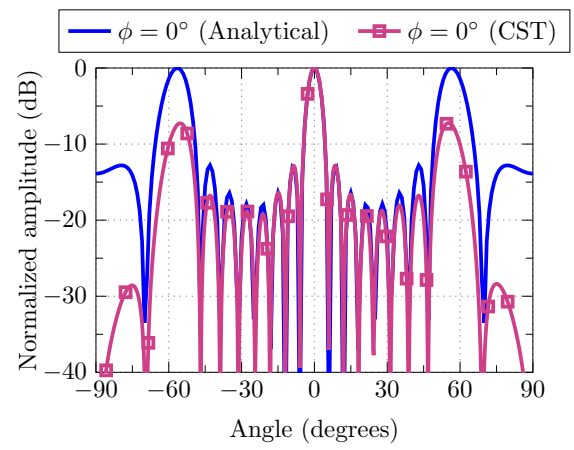

(a)

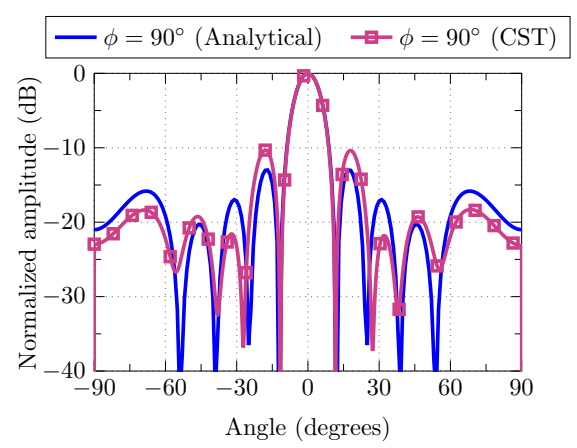

(b)

Figure 4.58: Comparison at $30 \mathrm{GHz}$ of the Array Factor calculated analytically and the radiation patterns simulated in CST. (Left) XZ-plane, (Right) YZplane.

lobes in the XZ-plane $\left(\phi=0^{\circ}\right)$. This behavior is also noticeable in the figures corresponding to the patterns for the TX band, see Fig. 4.58. The radiation null of the slot in $\mathrm{X}$-axis helps to reduce grating lobes slightly. Nevertheless, a great deal of improvement is required to be able to meet the specifications of SATCOM patterns.

\section{Ad-hoc solutions for grating lobes reduction}

Even if the feasibility of the antenna has been proven, there is still plenty of room for improvement. The interleaved arrangement provided certain advantages but TX patterns need to be improved. A well-known solution is to flare the apertures. In this way it is possible to reduce the grating lobes. Fig. 4.59 shows the $8 \times 8$ antenna where the radiating apertures have been flared.

Fig. 4.60 shows the comparison of the TX patterns between the $8 \times 8$ slotted antenna (Fig. 4.49) and the same antenna including horns as radiating element (Fig. 4.59). Horns reduce the lobes very slightly so they can not be considered a valid solution. Another alternative is to add a grid over the horns. Then, certain uniformity of the field at the TX band is achieved without modifying the field at the RX band.

Fig. 4.61 shows the new sheet that is placed over the horns. It is observed that the size of the RX apertures fits the RX horns. However, on the TX horns, the sheet subdivides into 4 slots, distributed in $2 \times 2$. This surface manages to uniform the field in the aperture. Comparing the patterns of the original design 


\section{CHAPTER 4. GAP WAVEGUIDE ARRAY ANTENNAS}

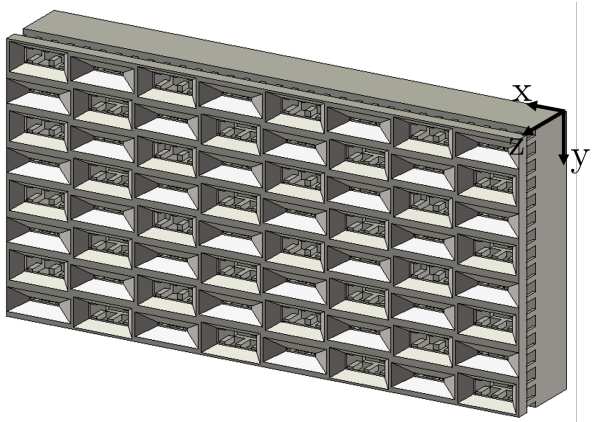

Figure 4.59: $8 \times 8$ Single-Layer Dual-Band Antenna.

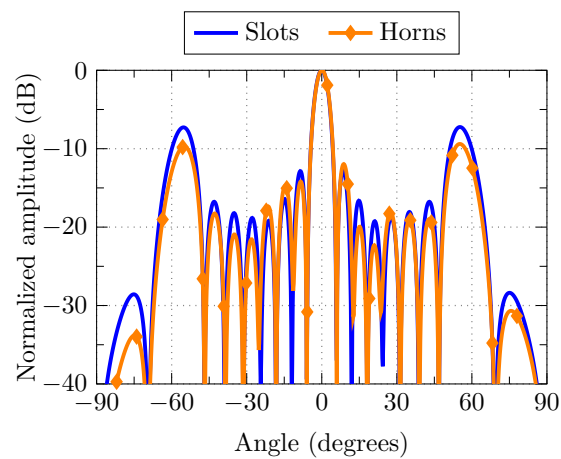

(a)

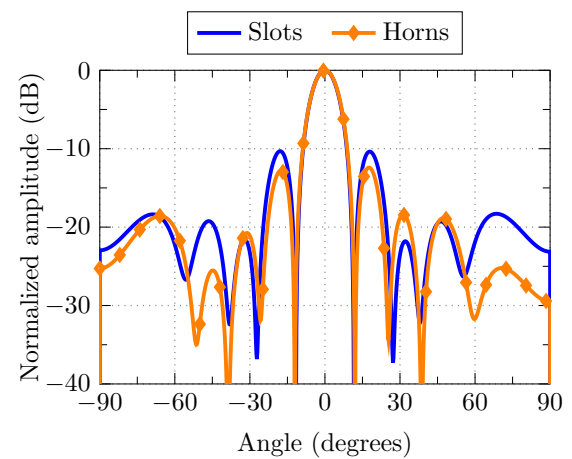

(b)

Figure 4.60: Comparison of radiation patterns at $30 \mathrm{GHz}$ using slots and using horns.

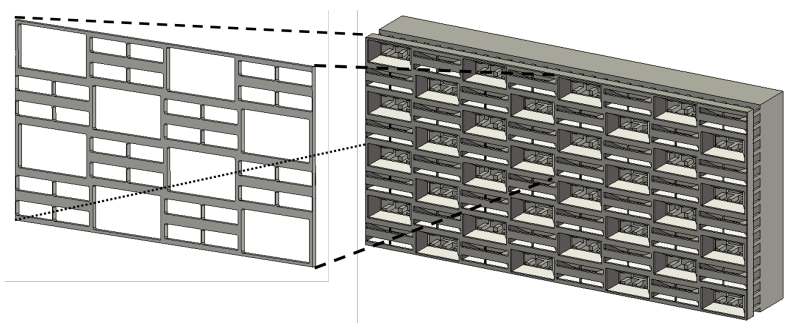

Figure 4.61: A grid is placed over the dual-band array to reduce the SLL.

and the one using horns, this grid achieves to reduce the SLL by more than 7 $\mathrm{dB}$ in the XZ-plane (Fig. 4.62) which was the most critical cut. 


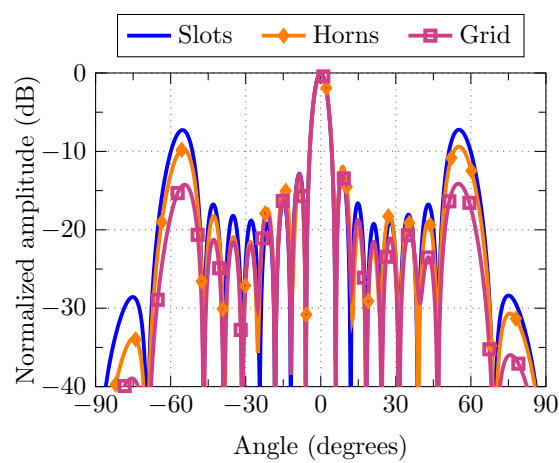

(a)

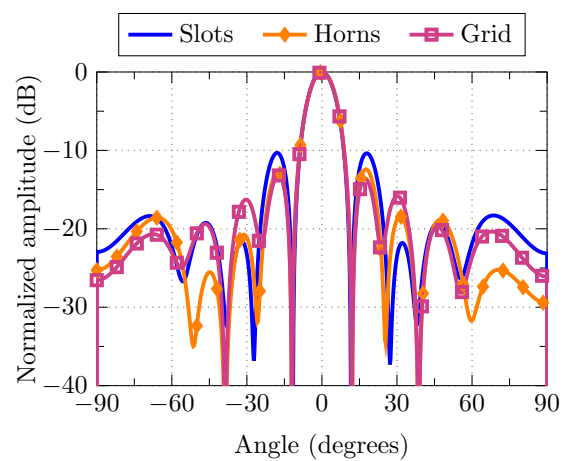

(b)

Figure 4.62: Comparison of radiation patterns at $30 \mathrm{GHz}$ using slots, horns and a grid.

\section{Conclusions}

Proposed single-layer dual-band array is composed by 64 apertures, 32 for $20 \mathrm{GHz}$ and 32 for $30 \mathrm{GHz}$. When employing just a single panel to hold apertures of two separate bands is important both the element arrangement and the spacing between them. A spacing of $12 \mathrm{~mm}$ (in the particular case studied) between apertures in the horizontal plane remains a bottleneck that leads to grating lobes in the XZ-plane at $30 \mathrm{GHz}$. A possible solution is a more compact network but there is also a constraint determined by the length of the slots at $20 \mathrm{GHz}$. Finally, horns and grids have been used to mitigate this effect and grating lobes have been reduced.

\section{6 $8 \times 8$ Dual-Band Array Antenna using Cylin- drical Cavities}

An alternative way of approaching dual-band antennas is through a multilayer antenna array, using the cylindrical cavities already seen in previous sections. For the case study, the working frequencies will be the same as in the previous array. The field distribution will be through an RGW-GGW network, which has proven to be the most efficient solution in this technology to distribute the signal in a compact way.

The proposed architecture is depicted in Fig. 4.63. It is very similar to the dual-polarized array. The distinctive feature, however, is in the intermediate layer where the radius of the cavities is larger, to account for the different 


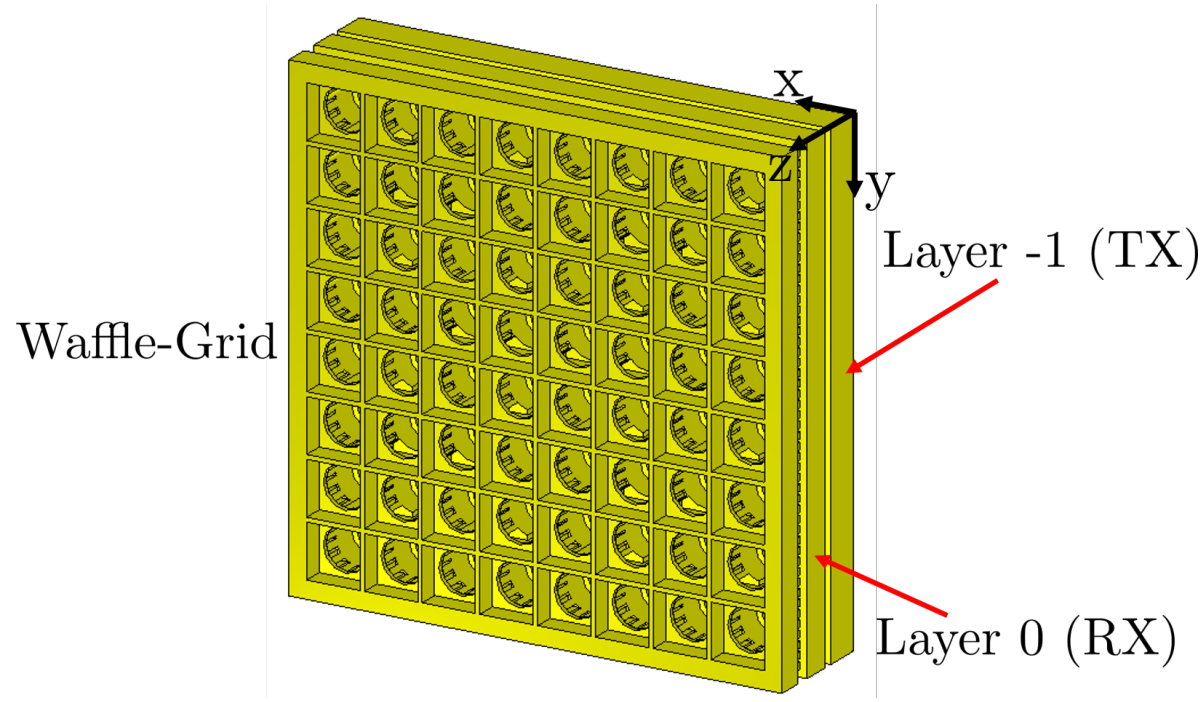

Figure 4.63: 3D model of an $8 \times 8$ antenna using cylindrical cavities.

working frequency. On top of the antenna, a waffle grid will be used. RX and TX fields are orthogonal to each other.

\section{TX Layer Design}

In Fig. 4.64 the lower layer is shown. From now on it will be referred to as Layer -1 . Here, all the GGW have the same dimensions in height and width. The total size of the dual-band antenna is $10 \mathrm{~cm} \times 10 \mathrm{~cm}$, instead of the $9 \mathrm{~cm} \times 9 \mathrm{~cm}$ of the dual-polarized antenna.

TX elements are spaced $12 \mathrm{~mm}, 1.2 \lambda_{0}$ apart, at the center frequency (30 $\mathrm{GHz}$ ). Obviously, this is not desirable but there is no option since these apertures must be perfectly aligned with the upper layers. In the upper layer the $\mathrm{RX}$ cavities show larger radius and require more room. This fact forces us to locate the TX cavities more spaced than desired. A $1.2 \lambda_{0}$ spacing, entails grating lobes in the TX pattern. However, the waffle-grid in the lid could mitigate this effect, as has already been demonstrated. All the relevant parameters of Layer -1 are shown in Table 4.9. 


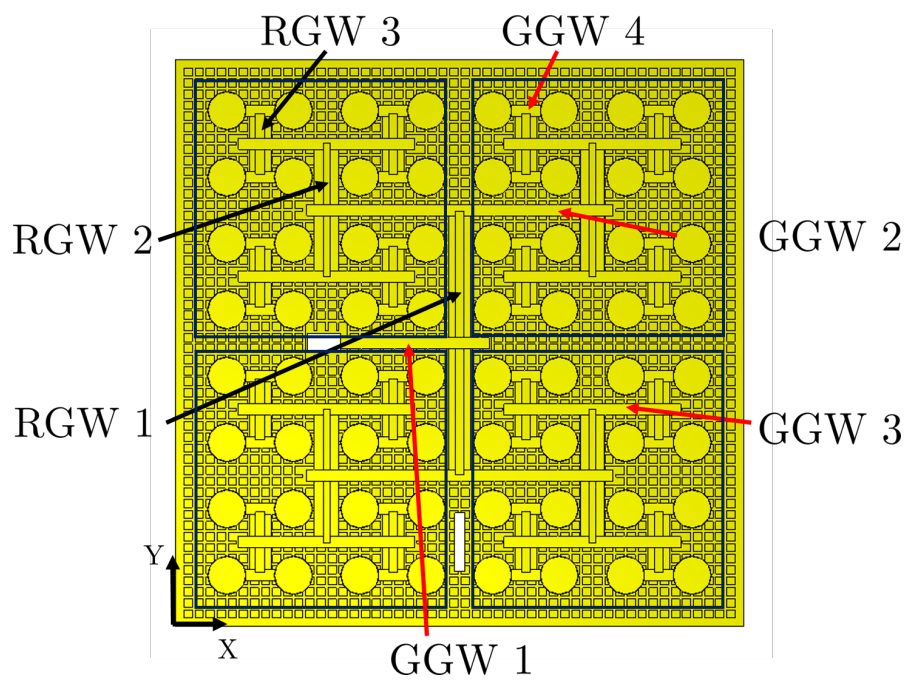

Figure 4.64: Top view of the lower layer (Layer -1 ). The different waveguides are indicated.

\begin{tabular}{|c|c|c|}
\hline \multicolumn{3}{|c|}{ Feeding Layer (TX) } \\
\hline Component & Parameter & Value $\mathbf{( m m}$ ) \\
\hline \multirow{2}{*}{ Nails } & $w \times h$ & $1.5 \times 2$ \\
& Period & 2 \\
\hline RGW (1) & \multirow{2}{*}{ Dimensions } & $2 \times 1.6$ \\
\cline { 1 - 1 } RGW (2) & & $1.5 \times 1.2$ \\
\cline { 1 - 1 } RGW (3) & $(h \times w)$ & $1.6 \times 1.6$ \\
\cline { 1 - 1 } GGW (all) & & $5.5 \times 2$ \\
\hline \multirow{2}{*}{ Cavity } & Radius & 3.42 \\
\cline { 3 - 3 } & Height & 5.9 \\
\hline
\end{tabular}

Table 4.9: Dimensions of the feeding layer (TX).

\section{RX Layer Design}

The intermediate layer (Layer 0) is responsible for the radiation at the RX band. The cylindrical cavities work at $20 \mathrm{GHz}$, therefore they have larger diameter than the cavities in Layer -1 .

Both layers are coupled by a circular aperture equal to the radius of the TX cavity. In this case a longitudinal slot is not used since both cavities are mutually decoupled because they operate at widely separated frequencies. Since the RX cavity has a larger radius, TX field can propagate through the 


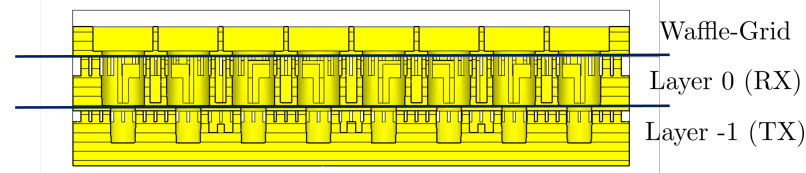

(a)

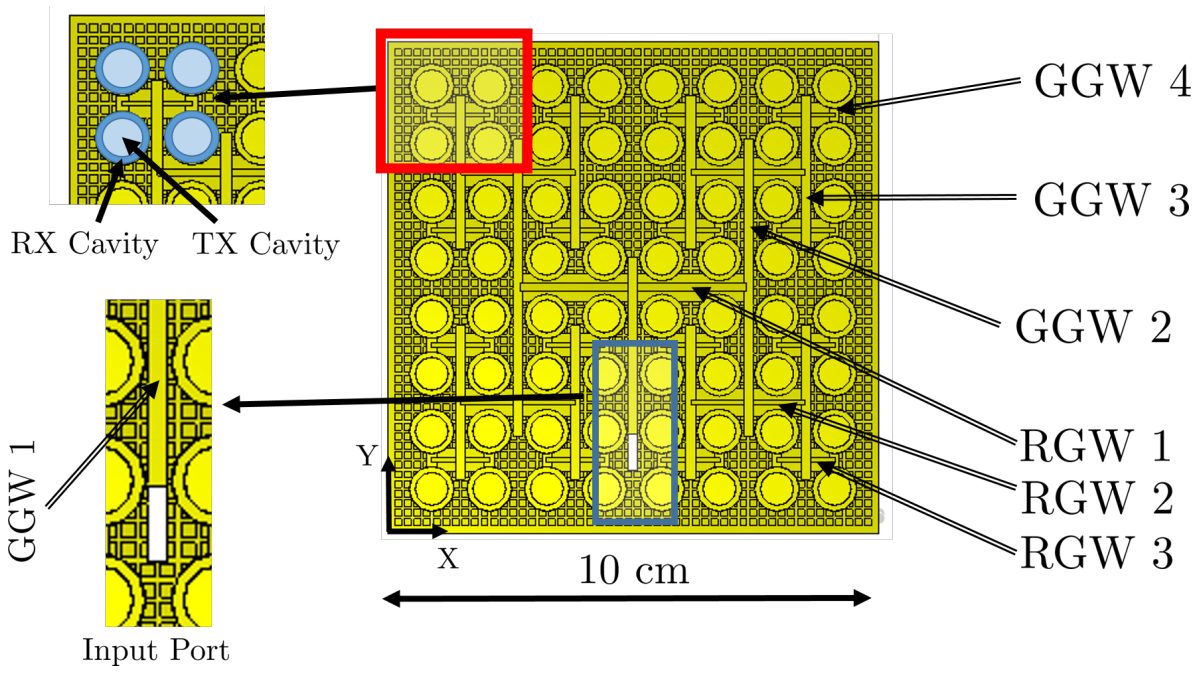

(b)

Figure 4.65: (a) Side view of the antenna. The different radius of TX and RX cavities are observed. (b) Front view of Layer 0 . The fundamental parts of the network are indicated by arrows.

\begin{tabular}{|c|c|c|}
\hline \multicolumn{3}{|c|}{ Feeding Layer (RX) } \\
\hline Component & Parameter & Value $\mathbf{( m m})$ \\
\hline \multirow{2}{*}{ Nails } & $w \times h$ & $1.5 \times 3.5$ \\
& Period & 2 \\
\hline RGW (1) & \multirow{2}{*}{ Dimensions } & $3.2 \times 1.7$ \\
\cline { 1 - 1 } RGW (2) & & $3.2 \times 1.1$ \\
\cline { 1 - 1 } RGW (3) & & $2.5 \times 0.9$ \\
\cline { 1 - 1 } GGW (all) & & $8.5 \times 2$ \\
\hline \multirow{2}{*}{ Cavity } & Radius & 4.7 \\
\cline { 3 - 3 } & Height & 9 \\
\hline
\end{tabular}

Table 4.10: Dimensions of the feeding layer (RX). 
RX cylinder. Interestingly, the orthogonality of the propagated components increases the decoupling between both layers. Fig. 4.65 details the fundamental parts of this intermediate layer. Again the distribution network consists of a combination of $4 \mathrm{GGW}$ and $3 \mathrm{RGW}$ of different dimensions. Input port and a $2 \times 2$ subarray of the antenna are also shown in detail. The TX input port crosses the lower layer to reach the intermediate level. As for the cylindrical cavities, they are stacked, being the TX cavity highlighted in light blue while the RX cavity is shown in dark blue. A table gathering the values of each dimension is shown in 4.10 .

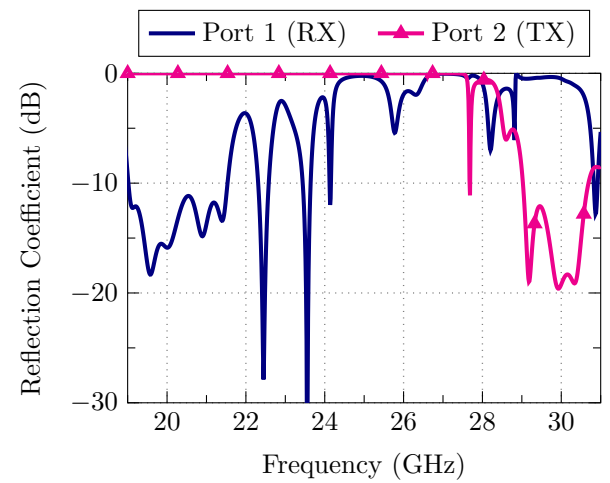

Figure 4.66: Simulated reflection coefficient of the dual-band antenna using cylindrical cavities.

\section{Preliminary results}

Some preliminary results are shown below. First, the reflection coefficient of the complete $8 \times 8$ antenna is shown in Fig. 4.66. The two RX-TX bands are clearly marked. The blue line is the reflection coefficient for port 1 , which feeds the intermediate layer. The RX band is below $-10 \mathrm{~dB}$ from $19 \mathrm{GHz}$ to 21.5 $\mathrm{GHz}$, therefore covering beyond the band of interest, from $19.7 \mathrm{GHz}$ to 21.2 $\mathrm{GHz}$. The pink line represents the reflection coefficient obtained at port 2, which is the one that feeds the lower layer. The $\mathrm{S}_{11}$-parameter is lower than $-10 \mathrm{~dB}$ from $29 \mathrm{GHz}$ to $30.6 \mathrm{GHz}$, therefore obtaining more than $5 \%$ of relative bandwidth.

In Fig. 4.67 radiation patterns for XZ- and YZ-planes for both bands are shown. As expected, grating lobes show up in the XZ-plane for the TX band. The rest of the patterns look fairly stable with frequency patterns, corresponding to the uniform illumination of the apertures. 


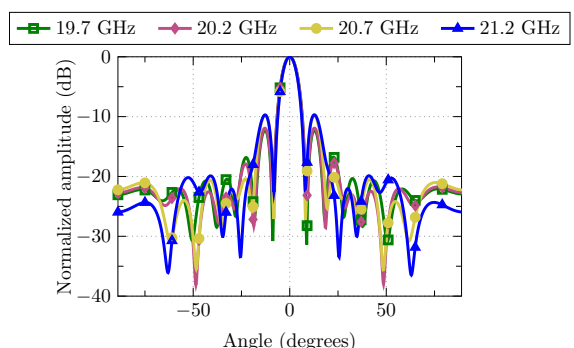

(a)

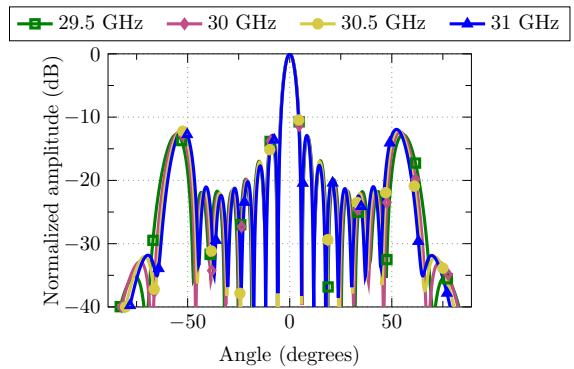

(c)

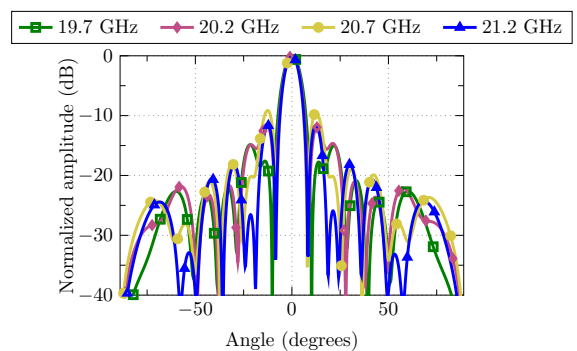

(b)

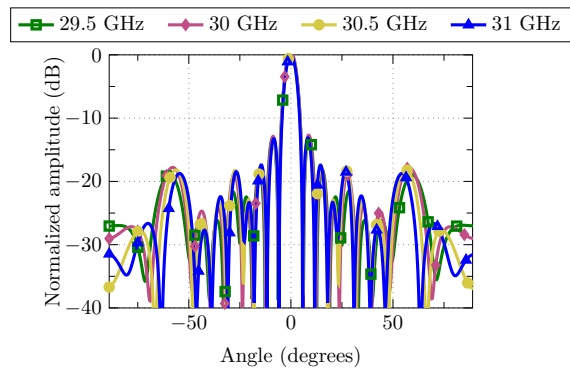

(d)

Figure 4.67: Simulated radiation patterns for several frequencies: (a) Simulated XZ-plane pattern (RX). (b) Simulated YZ-plane pattern (RX). (c) Simulated XZ-plane pattern (TX).(d) Simulated YZ-plane pattern (TX).

\section{Conclusions}

A $8 \times 8$ dual-band antenna array using cylindrical cavities has been presented. Distance between apertures in the horizontal plane is similar to the single-layer array $(12 \mathrm{~mm})$. Now, the radiating elements stacked instead of interleaved. Lower layer radiates at $30 \mathrm{GHz}$ and the intermediate layer at $20 \mathrm{GHz}$. A waffle grid is used as a lid to uniform the field in the apertures. Interestingly, the multilayer structure could be extended up to 4 levels. In that case, the two orthogonal polarization at $20 \mathrm{GHz}$ and $30 \mathrm{GHz}$ might be achieved. This would lead to an antenna capable of radiating either LHCP or RHCP at 20 and 30 $\mathrm{GHz}$. The drawback in that case would be its relatively high profile, around 8 $\mathrm{cm}$ in total. 


\title{
Chapter 5
}

\section{Conclusions and Future Work}

\author{
"Our responsibility is to do what \\ we can, learn what we can, \\ improve the solutions, and pass \\ them on."
}

— Richard Feynman

The objectives established at the beginning of the thesis have been approached and worked in depth until they have been accomplished within a expected grade of success. During these years, several new concepts and findings have shown up. From the perspective obtained at this point of the thesis, it is worth stopping and looking back at the work done.

In Chapter 1, a state of the art on those high-gain antennas at mm-wave band related to the scope of the Thesis was made. Some features of antennas reviewed are desirable for SATCOM. These are low-profile, low-cost, high-gain or beam-steering capability (mechanical, hybrid or electronic). In addition, a deep review regarding Gap Waveguide technology was carried out. From this broad research work it is concluded that:

- As the mm-waves are capable of transmitting large amounts of data with greater efficiency, they hold a major application across the telecommunication industry. Intensive research and development activities and investments from the private and public sectors is being carried out.

- Satcom On-The-Move market has experienced a rapid growth in recent years. In situations where no terrestrial communication infrastructure 


\section{CHAPTER 5. CONCLUSIONS AND FUTURE WORK}

exists (e.g. disaster scenarios), SOTM systems represent one of the best solutions. SOTM is a strong candidate in many other applications such as mobile TV, public security, rescue, maritime, aeronautical and military applications.

- There exists solutions based on parabolic reflectors, waveguide arrays, lenses, reflectarrays or horn arrays meeting several SATCOM needs, though there is still room for new contributions. In recent years, GW technology has proven to be a candidate able to provide high-gain low-profile antennas. In addition, the GW prevents leakage losses thanks to its inherent contactless shielding.

In Chapter 2, several printed and full-metal high impedance surfaces have been overviewed. The role of HIS on GW has been shown. Then, those corporate feeding networks based on Gap Waveguide that were used or developed through this thesis haven been analyzed. The following are the conclusions in this regard:

- There are different periodic structures such as mushroom-type, uniplanar, zig-zag wires or holey glide-symmetry that can be used to achieve a parallel-plate stopband for wave propagation. However, some of these technologies are printed while others are bulky, which limits their use in high-gain and low-profile arrays.

- The contactless feature of GW is a key aspect of the technology. To understand its potential, a comparative study between the GGW and RW performance was done. This contactless feature is key for multilayer antennas at the mm-wave band. As the joints across the waveguides must be a perfect metal to metal contact, even a minimum air gap can greatly spoil the device performance. Using the GW ensures a good assembly in large arrays.

- Existing GW networks use either GGW or RGW. A new network combining both has been presented. This network provides in-phase power division with symmetrical amplitude distribution. The primary advantage to combine these two types of waveguides is that they introduce a very profitable versatility in the design of corporate-feed networks. Whereas RGW leads to symmetrical networks, GGW provides a more compact structure.

Chapter 3 is devoted to develop radiating elements suitable for GW Arrays. Main conclusions extracted along the process are:

- The coaxial cavities have opened new possibilities in the implementation of more compact arrays. To date, most contributions of GW antennas in 
mm-wave followed a similar 3-layer scheme. Now, the versatility of the coaxial cavities have enabled single-layer architectures in GW.

- The capability of housing resonant cavities at different frequencies in the same bed of nails has also been demonstrated. Nevertheless, there is an obvious limitation in obtaining single-layer dual-band arrays using coaxial cavities. By sharing the same level, the slots at two widely separated bands leads to the appearance of grating lobes. Today it seems unfeasible to achieve a single-layer dual-band antenna with as good performance as that of a two dedicated panel arrays.

- The ability of cylindrical cavities to be placed in a stacked fashion has allowed to design circularly-polarized, dual-polarized and dual-band antennas, all of them quite desirable in SATCOM applications.

- The spacing between circular apertures is constrained by their radius, which is frequency dependent. Only a combined RGW-GGW network has proved to be a viable feeding of this type of radiating elements.

In Chapter 4, each of the elements detailed above are connected together to configure different kinds of arrays. From the results obtained it can be affirmed that:

- The coaxial cavities are more attractive for very low-profile linearlypolarized arrays. The cylindrical cavities, on the other hand, partially sacrifice the low profile feature in exchange for providing circular, dualpolarized or dual-band antenna arrays.

- The additive manufacturing will become a viable and economical alternative to conventional techniques such as milling. While milling performance is still better, the boom of additive manufacturing will soon reach enough accuracy to meet specifications. On the other hand, roughness is an aspect to be taken into account in $3 \mathrm{D}$ manufacturing in the mm-wave band, and can be very limiting in terms of losses.

- There exist very few high-gain, lightweight, low-cost and low-profile array antennas in the mm-wave band, despite being a demand in the SATCOM market. Although the GW antenna arrays contributed throughout this thesis are not yet integrated in a complete system, they are competitive approaches covering needs not met before.

\section{Future work}

This Thesis has reached significant entity to be finished here. However, as any research work, several issues remain open. Some future work lines have arisen 
during the research process as issues to solve or to improve, whereas others are its natural continuation. All of them are compiled in the following list:

- Due to the problems encountered to properly measure the $16 \times 16 \mathrm{~V}$-band Array Antenna, some features such as the polarization purity is pending validation.

- The waffle-grid has proven to be an effective solution to reduce grating lobes in antennas using cylindrical cavities. Other grids can be evaluated in order to find the best possible lid.

- The dual-band single-layer antenna still has some more milestones to cover. Although the reflection coefficient meets specification for both frequency bands, the radiation patterns can be improved. Grids able to reduce the grating lobes have been presented but better solutions must be investigated, even resorting to printed structures. In addition, the manufacture and measurement of the antenna is still pending.

- The dual-band antenna using cylindrical cavities has not been manufactured either. The architecture is very similar to the dual-polarized antenna. The promising performance found in simulations remains to be demonstrated experimentally.

- The use of cylindrical cavities would allow us to stack 4 layers, 2 for RX and 2 more for TX, therefore achieving a dual-polarized dual-band antenna. A future work would evaluate the viability of such solution or explore other alternatives to achieve that performance, especially appealing for SATCOM terminals.

- One valuable feature, not addressed in this thesis, involves the implementations of beam-steering mechanisms, either electronic or hybrid electronicmechanic. The contactless feature of the GW technology becomes a crucial property to exploit for that objective. The steering property would definitely provide the desired low-profile characteristic for SOTM terminals. 


\section{Appendix A}

\section{Technical Results}

\section{A.1 JCR Indexed journals}

- M. Ferrando-Rocher, J. I. Herranz-Herruzo, A. Valero-Nogueira, B. Bernardo, A.U. Zaman and J. Yang, " $8 \times 8$ Ka-Band Dual-Polarized Array Antenna based on Gap Waveguide Technology," Submitted for publication: IEEE Transactions on Antennas and Propagation, 2018.

- M. Ferrando-Rocher, J. I. Herranz-Herruzo, A. Valero-Nogueira, and A. Vila-Jiménez, "Single-layer Circularly-polarized Ka-band antenna using Gap Waveguide technology," IEEE Transactions on Antennas and Propagation, 2018.

- M. Ferrando-Rocher, J. I. Herranz, A. Valero-Nogueira, and B. Bernardo, "Performance assessment of gap waveguide array antennas: CNC milling vs. 3D printing," IEEE Antennas and Wireless Propagation Letters, 2018.

- E. Rajo-Iglesias, M. Ferrando-Rocher, and A. U. Zaman, "Gap waveguide technology for millimeter-wave antenna systems," IEEE Communications Magazine, vol. 56, no. 7, 2018.

- M. Baquero-Escudero, A. Valero-Nogueira, M. Ferrando-Rocher, B. Bernardo, and V. E. Boria, "Compact Combline Filter Embedded in a Bed of Nails," Submitted for publication: IEEE Transactions on Microwave Theory and Techniques, 2018.

- J. I. Herranz-Herruzo, A. Valero-Nogueira, M. Ferrando-Rocher, B. Bernardo, A. Vila, and R. Lenormand, "Low-cost Ka-band switchable 
RHCP/LHCP antenna array for mobile SATCOM terminal," IEEE Transactions on Antennas and Propagation, vol. 66, no. 5, pp. 2661-2666, 2018.

- M. Ferrando-Rocher, J. I. Herranz-Herruzo, A. Valero-Nogueira, and V. M. Rodrigo, "Circularly polarized slotted waveguide array with improved axial ratio performance," IEEE Transactions on Antennas and Propagation, vol. 64, no. 9, pp. 4144-4148, 2016.

\section{A.2 International Conferences}

- M. Ferrando-Rocher, J. I. Herranz-Herruzo, A. Valero-Nogueira, and M. Baquero-Escudero, "Flatness enhancement of gap waveguide slot arrays using a ribbed-grid plate," in IEEE International Symposium on Antennas and Propagation APS-URSI 2018, Boston, United States 8-13 July 2018 .

- M. Ferrando-Rocher, A. Valero-Nogueira, J. I. Herranz-Herruzo, and D. Sanchez-Escuderos, "Single-layer dual-band subarray for $20 / 30 \mathrm{GHz}$ using gap waveguide technology," in IEEE International Symposium on Antennas and Propagation APS-URSI 2018, Boston, United States 8-13 July 2018 .

- D. Sanchez-Escuderos, M. Ferrando-Rocher, A. Valero-Nogueira, and J. I. Herranz-Herruzo, "Linear to circular FSS transformer for dualpolarized applications," in IEEE International Symposium on Antennas and Propagation APS-URSI 2018, Boston, United States 8-13 July 2018

- M. Ferrando-Rocher, D. Sánchez-Escuderos, J. I. Herranz-Herruzo, and A. Valero-Nogueira, "Design of broadband gap waveguide transitions for millimeter-wave antenna arrays," in 48th European Microwave Conference (EuMC), 2018. IEEE, 2018.

- M. Ferrando-Rocher, A. Valero-Nogueira, J. I. Herranz-Herruzo, and B. Bernardo-Clemente, "8 x 8 Single-layer 30-GHz antenna with a combined ridge-groove gap waveguide network," in 12th European Conference on Antennas and Propagation (EUCAP), London (United Kingdom) 9-13 April 2018. IEEE, 2018.

- D. Sanchez-Escuderos, M. Ferrando-Rocher, A. Valero-Nogueira, and J. I. Herranz-Herruzo, "Dual-polarized frequency selective surface for SOTM applications," in 12th European Conference on Antennas and Propagation (EUCAP), London (UK) 9-13 April 2018. IEEE, 2018. 
- M. Ferrando-Rocher, J. Herranz-Herruzo, A. Valero-Nogueira, and B. Bernardo-Clemente, "SATCOM on-the-move antenna with mechanically switchable circular polarization," in the Loughborough Antennas \& Propagation Conference (LAPC), Loughborough (United Kingdom) 12-13 November $201 \%$.

- M. Ferrando-Rocher, A. Zaman, J. Yang, and A. Valero-Nogueira, "A dual-polarized slotted-waveguide antenna based on gap waveguide technology," in 11th European Conference on Antennas and Propagation (EUCAP), Paris (France) 19-24 March 2017. IEEE, 2017, pp. 37263727 .

- M. Ferrando-Rocher, A. Valero-Nogueira, and J. I. Herranz-Herruzo, "New feeding network topologies for high-gain single-layer slot array antennas using gap waveguide concept," in 11th European Conference on Antennas and Propagation (EUCAP), Paris (France) 19-24 March $201 \%$. IEEE, 2017, pp. 1654-1657.

- M. Ferrando-Rocher, A. Valero-Nogueira, J. I. Herranz-Herruzo, and A. Berenguer, "V-band single-layer slot array fed by ridge gap waveguide," in IEEE International Symposium on Antennas and Propagation APS-URSI 2016, Fajardo, Puerto Rico 26 June-1 July 2016. IEEE, 2016, pp. 389-390.

- A. Berenguer, M. Baquero-Escudero, M. Ferrando-Rocher, B. Bernardo-Clemente, and V. E. Boria, "An effective post-manufactured tuning method for gap waveguide components," in IEEE International Symposium on Antennas and Propagation APS-URSI 2016, Fajardo, Puerto Rico 26 June-1 July 2016. IEEE, 2016, pp. 493-494.

- M. Ferrando-Rocher, A. Valero-Nogueira, J. I. Herranz-Herruzo, A. Berenguer, and B. Bernardo-Clemente, "Groove gap waveguides: A contactless solution for multilayer slotted-waveguide array antenna assembly," in 10th European Conference on Antennas and Propagation (EUCAP), Davos (Switzerland) 10-15 April 2018. IEEE, 2016, pp. 1-4.

- A. Berenguer, V. Fusco, M. Ferrando-Rocher, and V. E. Boria, "A fast analysis method for the groove gap waveguide using transmission line theory," in 10th European Conference on Antennas and Propagation (EUCAP), Davos (Switzerland) 10-15 April 2018. IEEE, 2016, pp. 1-5.

- J. Herranz-Herruzo, M. Ferrando-Rocher, A. Valero-Nogueira, R. Lenormand, A. Hirsch, J. Almeida, M. Arnaud, and L. Barthe, "LOCOMO 
Satcom terminal: A switchable RHCP/LHCP array antenna for on-themove applications in ka-band," in IEEE International Symposium on Antennas and Propagation APS-URSI 2015, Vancouver, Canada 19 -25 July 2015. IEEE, 2015, pp. 210-211.

- J. I. Herranz-Herruzo, A. Valero-Nogueira, M. Ferrando-Rocher, B. Bernardo-Clemente, R. Lenormand, A. Hirsch, J.-L. Almeida, M. Arnaud, and L. Barthe, "Low cost switchable RHCP/LHCP antenna for SOTM applications in Ka-band," in 9th European Conference on Antennas and Propagation (EUCAP), Lisbon (Portugal) 12-17 April 2015. IEEE, 2015, pp. 1-4.

- A. Valero-Nogueira, J. I. Herranz-Herruzo, M. Ferrando-Rocher, R. Lenormand, A. Hirsch, and J.-L. Almeida, "Switchable RHCP/LHCP slotted waveguide array antenna for satcom on-the-move applications in Ka-band," in 8th European Conference on Antennas and Propagation (EUCAP), The Hague (Netherlands) 6-11 April 2014. IEEE, 2014, pp. 2047-2051.

\section{A.3 National Conferences}

- M. Ferrando-Rocher, A. Valero-Nogueira, D. Sanchez-Escuderos, and J. I. Herranz-Herruzo, "Antena dual $(\mathrm{K} / \mathrm{Ka})$ de bajo perfil utilizando tecnoloía de guía gap." in XXXIII Simposium Nacional de la Unión Científica Internacional de Radio (URSI 2018) Granada, Spain, 2018.

- R. Caballero-Nagore, J. Teniente-Vallinas, M. Ferrando-Rocher, and A. Valero-Nogueira, " $440 \mathrm{GHz}$ fabrication of a slotted waveguide antenna based on gap waveguide technology," in XXXIII Simposium Nacional de la Unión Científica Internacional de Radio (URSI 2018) Granada, Spain, 2018.

- M. Ferrando-Rocher, J. I. Herranz-Herruzo, A. Valero-Nogueira, and V.-J. Antonio, "Single-layer circularly-polarized ka-band antenna using gap waveguide technology," in XXXII Simposium Nacional de la Unión Científica Internacional de Radio (URSI 2017) Cartagena, Spain, 2017.

- M. Ferrando-Rocher, J. I. Herranz-Herruzo, A. Valero-Nogueira, and B. Bernardo-Clemente, "Antena plana con polarización circular para comunicaciones por satélite en banda Ka: desafíos y resultados," in XXXI Simposium Nacional de la Unión Científica Internacional de Radio (URSI 2016) Madrid, Spain, 2016. 
- M. Ferrando-Rocher, J. I. Herranz-Herruzo, A. Valero-Nogueira, B. Bernardo-Clemente, and V.-J. Antonio, "Diseño y medidas de una antena TX con polarización en banda Ka y distribución Taylor 25 dB, " in $X X X$ Simposium Nacional de la Unión Científica Internacional de Radio (URSI 2015) Pamplona, Spain, 2015.

- M. Ferrando-Rocher, J. I. Herranz-Herruzo, A. Valero-Nogueira, M.G. Sara, and V.-J. Antonio, "Array de guías ranuradas con polarización circular conmutable para aplicaciones satcom en movimiento en banda Ka," in XXIX Simposium Nacional de la Unión Científica Internacional de Radio (URSI 2014) Valencia, Spain, 2014.

\section{A.4 Other publications}

\section{A.4.1 JCR Indexed journals}

- T. Makdissy, R. Gillard, E. Fourn, M. Ferrando-Rocher, E. Girard, H. Legay, and L. Le Coq, "Phoenix' reflectarray unit cell with reduced size and inductive loading," IET Microwaves, Antennas \& Propagation, vol. 10, no. 12, pp. 1363-1370, 2016.

\section{A.5 Projects}

- NEW ANTENNAS FOR SATCOM ON THE MOVE IN THE KA-BAND. (UPV, 2017-2019)

Funded by Ministry of Economy, Industry and Competitiveness (€112.000 budget).

- mmWAVES IN LTCC TECHNOLOGY FOR ANTENNA SYSTEMS 2020. (UPV, 2015-2017)

Funded by Ministry of Economy, Industry and Competitiveness (€261.000 budget).

\section{- LOW COST AND COMPACT KA-BAND MOBILE SATCOM TERMINAL (UPV, 2013-2016)}

Funded by ESA and developed in collaboration with the companies Thales Alenia Space, TTI Norte, Vectra Wave and iXBlue (€159.000 budget). 


\section{A.6 Patents}

- A. Valero-Nogueira, M. Ferrando-Rocher and J. I. Herranz-Herruzo, "Antena de alta ganancia dual en frecuencia en banda Ka", European Patent, PCT/ES2018/070645 , October 2018

\section{A.7 Awards}

\section{- Young Scientist Award URSI 2017}

Contribution: Single-Layer Circularly-Polarized Ka-band Antenna in Gap Waveguide

- Jury prize to the best oral presentation (Pitch Challenge) in the II Meeting of PhD Students. Doctoral School UPV.

- Audience prize to the best oral presentation (Pitch Challenge) in the II Meeting of PhD Students. Doctoral School UPV. 


\section{References}

[1] M. Marcus and B. Pattan, "Millimeter wave propagation: spectrum management implications," IEEE Microwave Magazine, vol. 6, no. 2, pp. 54$62,2005$.

[2] E. Rajo-Iglesias, M. Ferrando-Rocher, and A. U. Zaman, "Gap Waveguide Technology for Millimeter-Wave Antenna Systems," IEEE Communications Magazine, vol. 56, no. 7, pp. 14-20, 2018.

[3] P.-S. Kildal, E. Alfonso, A. Valero-Nogueira, and E. Rajo-Iglesias, "Local metamaterial-based waveguides in gaps between parallel metal plates," IEEE Antennas and Wireless Propagation Letters, vol. 8, pp. 84-87, 2009.

[4] P.-S. Kildal, A. U. Zaman, E. Rajo-Iglesias, E. Alfonso, and A. ValeroNogueira, "Design and experimental verification of ridge gap waveguide in bed of nails for parallel-plate mode suppression," IET Microwaves, Antennas 83 Propagation, vol. 5, no. 3, pp. 262-270, 2011.

[5] "ETSI - New Industry Specification Group on Millimetre Wave Transmission at ETSI," https://www.etsi.org/, (Accessed on 03/01/2018).

[6] Y. Peng, "On-Chip Low Profile Metamaterial Antennas for Wireless Millimetre-wave Communications," 2012.

[7] P. Adhikari, "Understanding millimeter wave wireless communication," Loea Corporation, 2008.

[8] "FCC to Release More Millimeter Wave Spectrum," https://spectrummattersindeed.blogspot.com.es/2017/10/ fcc-to-release-more-millimeter-wave.html.

[9] L. Rayleigh, "On the passage of electric waves through tubes, or the vibrations of dielectric cylinders," The London, Edinburgh, and Dublin Philosophical Magazine and Journal of Science, vol. 43, no. 261, pp. 125132, 1897. 


\section{REFERENCES}

[10] C. R. Anderson and T. S. Rappaport, "In-building wideband partition loss measurements at 2.5 and $60 \mathrm{GHz}$," IEEE transactions on wireless communications, vol. 3, no. 3, pp. 922-928, 2004.

[11] R. C. Daniels, J. N. Murdock, T. S. Rappaport, and R. W. Heath, "60 GHz wireless: Up close and personal," IEEE Microwave magazine, vol. 11, no. 7, pp. 44-50, 2010.

[12] E. Perahia, C. Cordeiro, M. Park, and L. L. Yang, "IEEE 802.11 ad: Defining the next generation multi-Gbps Wi-Fi," in Consumer Communications and Networking Conference (CCNC), 2010 7th IEEE. IEEE, 2010, pp. 1-5.

[13] L. Eastwood, S. Migaldi, Q. Xie, and V. Gupta, "Mobility using IEEE 802.21 in a heterogeneous IEEE 802.16/802.11-based, IMT-advanced (4G) network," IEEE Wireless Communications, vol. 15, no. 2, 2008.

[14] "Institut fur Telekommunikationssysteme: Neighbor Discovery and Tracking in mm-Wave Networks," https://www.tkn.tu-berlin.de/.

[15] J. V. Evans and A. Dissanayake, "The prospects for commercial satellite services at Q-and V-band," Space communications, vol. 15, no. 1, pp. $1-19,1998$.

[16] L. Marcellini, R. L. Forti, G. Bellaveglia, A. Colasante, F. Maggio, and O. Russo, "Multi-reflector multi-band antennas for airborne and maritime broadband applications," in Antennas and Propagation in Wireless Communications (APWC), 2013 IEEE-APS Topical Conference on. IEEE, 2013, pp. 1131-1134.

[17] S. Y. Eom, S. H. Son, Y. B. Jung, S. I. Jeon, S. A. Ganin, A. G. Shubov, A. K. Tobolev, and A. V. Shishlov, "Design and test of a mobile antenna system with tri-band operation for broadband satellite communications and DBS reception," IEEE Transactions on antennas and propagation, vol. 55, no. 11, pp. 3123-3133, 2007.

[18] R. Liu, C. Ji, Z. Zhao, and T. Zhou, "Metamaterials: reshape and rethink," Engineering, vol. 1, no. 2, pp. 179-184, 2015.

[19] N. Chahat, J. Sauder, M. Thomson, R. Hodges, and Y. Rahmat-Samii, "CubeSat deployable Ka-band reflector antenna for deep space missions," in Antennas and Propagation $\&$ USNC/URSI National Radio Science Meeting, 2015 IEEE International Symposium on. IEEE, 2015, pp. 2185-2186. 
[20] S. Gao, Y. Rahmat-Samii, R. E. Hodges, and X.-X. Yang, "Advanced Antennas for Small Satellites," Proceedings of the IEEE, vol. 106, no. 3, pp. 391-403, 2018.

[21] Y. Zhai, D. Xu, and Y. Zhang, "Ka-Band Lightweight High-Efficiency Wideband 3D Printed Reflector Antenna," International Journal of Antennas and Propagation, vol. 2017, 2017.

[22] D. Sánchez-Escuderos, M. Cabedo-Fabrés, E. Antonino-Daviu, and M. Ferrando-Bataller, "Extended low-profile planar lens antenna with multilayer metallic-hole array," in Antennas and Propagation (EuCAP), 2015 9th European Conference on. IEEE, 2015, pp. 1-5.

[23] O. Quevedo-Teruel, J. Miao, M. Mattsson, A. Algaba-Brazalez, M. Johansson, and L. Manholm, "Glide-Symmetric Fully Metallic Luneburg Lens for 5G Communications at K a-Band," IEEE Antennas and Wireless Propagation Letters, vol. 17, no. 9, pp. 1588-1592, 2018.

[24] J. R. Costa, C. A. Fernandes, G. Godi, R. Sauleau, L. Le Coq, H. Legay et al., "Compact Ka-band lens antennas for LEO satellites," 2008.

[25] X. Wu, G. V. Eleftheriades, and T. E. van Deventer-Perkins, "Design and characterization of single-and multiple-beam mm-wave circularly polarized substrate lens antennas for wireless communications," IEEE Transactions on Microwave Theory and Techniques, vol. 49, no. 3, pp. 431-441, 2001.

[26] Y. J. Cheng, W. Hong, K. Wu, Z. Q. Kuai, C. Yu, J. X. Chen, J. Y. Zhou, and H. J. Tang, "Substrate integrated waveguide (SIW) Rotman lens and its Ka-band multibeam array antenna applications," IEEE Transactions on antennas and propagation, vol. 56, no. 8, pp. 2504-2513, 2008.

[27] J. Thornton, "Wide-scanning multi-layer hemisphere lens antenna for Ka band," IEE proceedings-microwaves, antennas and propagation, vol. 153, no. 6 , pp. 573-578, 2006.

[28] J. S. Silva, E. B. Lima, J. R. Costa, C. A. Fernandes, and J. R. Mosig, "Tx-Rx lens-based satellite-on-the-move Ka-band antenna," IEEE Antennas and Wireless Propagation Letters, vol. 14, pp. 1408-1411, 2015.

[29] J. S. Silva, M. García-Vigueras, M. Esquius-Morote, J. R. Costa, C. A. Fernandes, and J. R. Mosig, "Lens-based Ka-band antenna system using planar feed," in Antennas and Propagation (EuCAP), 2015 9th European Conference on. IEEE, 2015, pp. 1-4. 
[30] — - "A planar feed for SOTM Ka-band lens antennas," in Antennas and Propagation 8 USNC/URSI National Radio Science Meeting, 2015 IEEE International Symposium on. IEEE, 2015, pp. 226-227.

[31] D. Santilián-Haro, E. Antonino-Daviu, D. Sánchez-Escuderos, and M. Ferrando-Bataller, "Single layer metasurface lens antenna," in $A n$ tennas and Propagation (EUCAP), 2017 11th European Conference on. IEEE, 2017, pp. 1134-1138.

[32] D. Sanchez-Escuderos, H. C. Moy-Li, E. Antonino-Daviu, M. CabedoFabres, and M. Ferrando-Bataller, "Microwave planar lens antenna designed with a three-layer frequency-selective surface," IEEE Antennas and Wireless Propagation Letters, vol. 16, pp. 904-907, 2017.

[33] C. Pfeiffer and A. Grbic, "A printed, broadband Luneburg lens antenna," IEEE Transactions on Antennas and Propagation, vol. 58, no. 9, pp. 3055-3059, 2010.

[34] A. R. Weily and N. Nikolic, "Dual-polarized planar feed for low-profile hemispherical Luneburg lens antennas," IEEE Transactions on Antennas and Propagation, vol. 60, no. 1, pp. 402-407, 2012.

[35] J. Thornton, D. Smith, S. Foti, and Y. Jiang, "Reduced height Luneburg lens antennas for satellite communications-on-the-move," in Microwave Techniques (COMITE), 2015 Conference on. IEEE, 2015, pp. 1-4.

[36] C. Diallo, E. Girard, H. Legay, and R. Sauleau, "All-metal Ku-band Luneburg lens antenna based on variable parallel plate spacing Fakir bed of nails," in Antennas and Propagation (EUCAP), 2017 11th European Conference on. IEEE, 2017, pp. 1401-1404.

[37] A. A. Brazález, L. Manholm, M. Johansson, O. Quevedo-Teruel, and J. Miao, "Investigation of a Ka-band Luneburg lens made of a glidesymmetric holey structure," in Antennas and Propagation (ISAP), 2017 International Symposium on. IEEE, 2017, pp. 1-2.

[38] H. Legay, D. Bresciani, E. Labiole, R. Chiniard, E. Girard, G. Caille, D. Calas, R. Gillard, and G. Toso, "A $1.3 \mathrm{~m}$ facetted reflectarray in $\mathrm{Ku}$ band," in Antenna Technology and Applied Electromagnetics (ANTEM), 2012 15th International Symposium on. IEEE, 2012, pp. 1-4.

[39] P. De Vita, A. Freni, P. Pirinoli, and R. Zich, "A novel broadband singlelayer printed reflectarray antenna," in Antennas and Propagation Society International Symposium, 2007 IEEE. IEEE, 2007, pp. 1449-1452. 
[40] T. Smith, U. Gothelf, O. S. Kim, and O. Breinbjerg, "Design, manufacturing, and testing of a $20 / 30-\mathrm{GHz}$ dual-band circularly polarized reflectarray antenna," IEEE Antennas and Wireless Propagation Letters, vol. 12, pp. 1480-1483, 2013.

[41] J. A. Encinar, L. S. Datashvili, J. A. Zornoza, M. Arrebola, M. SierraCastañer, J. L. Besada-Sanmartin, H. Baier, and H. Legay, "Dualpolarization dual-coverage reflectarray for space applications," IEEE Transactions on Antennas and Propagation, vol. 54, no. 10, pp. 28272837, 2006.

[42] J. A. Encinar and J. A. Zornoza, "Broadband design of three-layer printed reflectarrays," IEEE Transactions on Antennas and Propagation, vol. 51, no. 7, pp. 1662-1664, 2003.

[43] J. A. Encinar, "Design of two-layer printed reflectarrays using patches of variable size," IEEE Transactions on Antennas and Propagation, vol. 49, no. 10, pp. 1403-1410, 2001.

[44] R. Florencio Díaz, J. A. Encinar Garcinuño, R. R. Boix, V. Losada, and G. Toso, "Reflectarray antennas for dual polarization and broadband telecom satellite applications," IEEE Transactions on antennas and propagation, vol. 63, no. 4, pp. 1234-1246, 2015.

[45] E. Carrasco, J. A. Encinar, and M. Barba, "Bandwidth improvement in large reflectarrays by using true-time delay," IEEE Transactions on Antennas and Propagation, vol. 56, no. 8, pp. 2496-2503, 2008.

[46] T. Chaloun, V. Ziegler, and W. Menzel, "Design of a dual-polarized stacked patch antenna for wide-angle scanning reflectarrays," IEEE Transactions on Antennas and Propagation, vol. 64, no. 8, pp. 33803390, 2016.

[47] G. Perez-Palomino, M. Barba, J. Encinar, R. Cahill, R. Dickie, and P. Baine, "Liquid crystal based beam scanning reflectarrays and their potential in SATCOM antennas," in 11th European Conf. Antennas and Propagation (EuCAP), Paris, France, 2017, pp. 3428-3431.

[48] Q. Luo and S. Gao, "Smart antennas for satellite communications on the move," in Antenna Technology: Small Antennas, Innovative Structures, and Applications (iWAT), 2017 International Workshop on. IEEE, 2017, pp. 260-263.

[49] H. Legay and L. Shafai, "New stacked microstrip antenna with large bandwidth and high gain," IEE Proceedings-Microwaves, Antennas and Propagation, vol. 141, no. 3, pp. 199-204, 1994. 


\section{REFERENCES}

[50] E. Nishiyama, M. Aikawa, and S. Egashira, "Stacked microstrip antenna for wideband and high gain," IEE Proceedings-Microwaves, Antennas and Propagation, vol. 151, no. 2, pp. 143-148, 2004.

[51] J. Huang, "A Ka-band circularly polarized high-gain microstrip array antenna," IEEE Transactions on antennas and propagation, vol. 43, no. 1, pp. 113-116, 1995.

[52] P. Sadri-Moshkenani, J. Rashed-Mohassel, and M. Shahabadi, "Microstrip Antenna Array Fed by a Low-Loss Gap-Waveguide Feed Network," IEEE Transactions on Antennas and Propagation, 2018.

[53] J. Liu, A. Vosoogh, A. U. Zaman, and J. Yang, "Design and fabrication of a high-gain $60-\mathrm{GHz}$ cavity-backed slot antenna array fed by inverted microstrip gap waveguide," IEEE Transactions on Antennas and Propagation, vol. 65, no. 4, pp. 2117-2122, 2017.

[54] K. Sakakibara, J. Hirokawa, M. Ando, and N. Goto, "High-gain and highefficiency single-layer slotted waveguide array for use in $22 \mathrm{GHz}$ band," Electronics Letters, vol. 32, no. 4, pp. 283-284, 1996.

[55] — - "Single-layer slotted waveguide arrays for millimeter wave applications," IEICE transactions on communications, vol. 79, no. 12, pp. $1765-1772,1996$.

[56] K. Sakakibara, Y.-i. Kimura, A. Akiyama, J. Hirokawa, M. Ando, and N. Goto, "Alternating phase-fed waveguide slot arrays with a singlelayer multiple-way power divider," IEE Proceedings-Microwaves, Antennas and Propagation, vol. 144, no. 6, pp. 425-430, 1997.

[57] M. Sano, J. Hirokawa, and M. Ando, "Single-layer corporate-feed slot array in the $60-\mathrm{GHz}$ band using hollow rectangular coaxial lines," IEEE Transactions on Antennas and Propagation, vol. 62, no. 10, pp. 50685076, 2014.

[58] M. Zhang, J. Hirokawa, and M. Ando, "Design of a double-layer slotted waveguide array with a partially corporate feed circuit installed in the bottom layer and its fabrication by diffusion bonding of laminated thin plates in 38GHz band," ISAP, Session: TB2, vol. 2, 2009.

[59] — - "Design of a partially-corporate feed double-layer slotted waveguide array antenna in $39 \mathrm{GHz}$ band and fabrication by diffusion bonding of laminated thin metal plates," IEICE transactions on communications, vol. 93, no. 10, pp. 2538-2544, 2010. 
[60] — - "Fabrication of a slotted waveguide array at $94 \mathrm{GHz}$ by diffusion bonding of laminated thin plates," in Antennas and Propagation Society International Symposium, 2009. APSURSI'09. IEEE. IEEE, 2009, pp. $1-4$.

[61] T. Tomura, J. Hirokawa, T. Hirano, and M. Ando, "A 45 Linearly Polarized Hollow-Waveguide 16×16-Slot Array Antenna Covering 71-86 GHz Band," IEEE Transactions on Antennas and Propagation, vol. 62, no. 10, pp. 5061-5067, 2014.

[62] Y. Miura, J. Hirokawa, M. Ando, Y. Shibuya, and G. Yoshida, "A 60GHz double-layer waveguide slot array with more than $32 \mathrm{dBi}$ and $80 \%$ efficiency over $5 \mathrm{GHz}$ bandwidth fabricated by diffusion bonding of laminated thin metal plates," in Antennas and Propagation Society International Symposium (APSURSI), 2010 IEEE. IEEE, 2010, pp. 1-4.

[63] J. Hirokawa, M. Zhang, and M. Ando, "94GHz single-layer slotted waveguide array by diffusion bonding of laminated thin plates," in 2008 Intl. Symp. Antennas Propagat., 2008.

[64] — , $94 \mathrm{GHz}$ fabrication of a slotted waveguide array antenna by diffusion bonding of laminated thin plates," in Sensors, 2009 IEEE. IEEE, 2009, pp. 907-911.

[65] M. Sano, J. Hirokawa, and M. Ando, "Design of a corporate-fed $2 \times 2-$ element slot array on a hollow rectangular coaxial line," in Electromagnetic Theory (EMTS), Proceedings of 2013 URSI International Symposium on. IEEE, 2013, pp. 606-607.

[66] A. Vosoogh, M. S. Sorkherizi, A. U. Zaman, J. Yang, and A. A. Kishk, "An Integrated Ka-Band Diplexer-Antenna Array Module Based on Gap Waveguide Technology With Simple Mechanical Assembly and No Electrical Contact Requirements," IEEE Transactions on Microwave Theory and Techniques, 2017.

[67] A. Farahbakhsh, D. Zarifi, and A. U. Zaman, "60-GHz Groove Gap Waveguide Based Wideband H-Plane Power Dividers and Transitions: For Use in High-Gain Slot Array Antenna," IEEE Transactions on Microwave Theory and Techniques, vol. 65, no. 11, pp. 4111-4121, 2017.

[68] D. Zarifi, A. Farahbakhsh, A. U. Zaman, and P.-S. Kildal, "Design and fabrication of a high-gain $60-\mathrm{GHz}$ corrugated slot antenna array with ridge gap waveguide distribution layer," IEEE Transactions on Antennas and Propagation, vol. 64, no. 7, pp. 2905-2913, 2016. 
[69] A. U. Zaman and P.-S. Kildal, "Different gap waveguide slot array configurations for mmwave fixed beam antenna application," in Antennas and Propagation (EuCAP), 2016 10th European Conference on. IEEE, 2016, pp. 1-4.

[70] P. Taghikhani, J. Yang, and A. Vosoogh, "High gain V-band planar array antenna using half-height pin gap waveguide," in Antennas and Propagation (EUCAP), 2017 11th European Conference on. IEEE, 2017, pp. $2758-2761$.

[71] P. Liu, Q. Hu, and Z. Chen, "E-band double layer cavity backed slot array antenna," in Antennas and Propagation (ISAP), 2017 International Symposium on. IEEE, 2017, pp. 1-2.

[72] A. U. Zaman and P.-S. Kildal, "Wide-band slot antenna arrays with single-layer corporate-feed network in ridge gap waveguide technology," IEEE Transactions on antennas and propagation, vol. 62, no. 6, pp. 29923001, 2014.

[73] _ - "Slot antenna in ridge gap waveguide technology," in Antennas and Propagation (EUCAP), 2012 6th European Conference on. IEEE, 2012, pp. 3243-3244.

[74] A. Vosoogh and P.-S. Kildal, "Corporate-fed planar 60-GHz slot array made of three unconnected metal layers using AMC pin surface for the gap waveguide," IEEE Antennas and Wireless Propagation Letters, vol. 15, pp. 1935-1938, 2016.

[75] A. Vosoogh, P.-S. Kildal, and V. Vassilev, "Wideband and High-Gain Corporate-Fed Gap Waveguide Slot Array Antenna With ETSI Class II Radiation Pattern in V-Band," IEEE Transactions on Antennas and Propagation, vol. 65, no. 4, pp. 1823-1831, 2017.

[76] Y. Li and K.-M. Luk, "Low-cost high-gain and broadband substrateintegrated-waveguide-fed patch antenna array for $60-\mathrm{GHz}$ band," IEEE Transactions on Antennas and Propagation, vol. 62, no. 11, pp. 5531$5538,2014$.

[77] A. Dadgarpour, B. Zarghooni, B. S. Virdee, and T. A. Denidni, "Millimeter-wave high-gain SIW end-fire bow-tie antenna," IEEE Transactions on Antennas and Propagation, vol. 63, no. 5, pp. 2337-2342, 2015.

[78] Y. J. Cheng, Y. X. Guo, and Z. G. Liu, "W-band large-scale high-gain planar integrated antenna array," IEEE Transactions on Antennas and Propagation, vol. 62, no. 6, pp. 3370-3373, 2014. 
[79] L. Wang, Y.-X. Guo, and W.-X. Sheng, "Wideband high-gain 60-GHz LTCC L-probe patch antenna array with a soft surface," IEEE transactions on antennas and propagation, vol. 61, no. 4, pp. 1802-1809, 2013.

[80] J. Wu, Y. J. Cheng, and Y. Fan, "Millimeter-wave wideband highefficiency circularly polarized planar array antenna," IEEE Transactions on Antennas and Propagation, vol. 64, no. 2, pp. 535-542, 2016.

[81] K. X. Wang and H. Wong, "A wideband millimeter-wave circularly polarized antenna with 3-D printed polarizer," IEEE Trans. Antennas Propag, vol. 65, no. 3, pp. 1038-1046, 2017.

[82] J. Cao, H. Wang, S. Mou, S. Quan, and Z. Ye, "W-Band High-Gain Circularly Polarized Aperture-Coupled Magneto-Electric Dipole Antenna Array With Gap Waveguide Feed Network," IEEE Antennas and Wireless Propagation Letters, vol. 16, pp. 2155-2158, 2017.

[83] J. Xi, B. Cao, H. Wang, and Y. Huang, "A novel 77 GHz circular polarization slot antenna using ridge gap waveguide technology," in Microwave Conference (APMC), 2015 Asia-Pacific, vol. 3. IEEE, 2015, pp. 1-3.

[84] T. Li and F. Fan, "Design of Ka-band $2 \times 2$ circular polarization slot antenna array fed by ridge gap waveguide," in 2017 Sixth Asia-Pacific Conference on Antennas and Propagation (APCAP). IEEE, 2017, pp. $1-3$.

[85] E. Garcia-Marin, J. L. Masa-Campos, and P. Sanchez-Olivares, "Diffusion Bonding Manufacturing of High Gain W-Band Antennas for 5G Applications," IEEE Communications Magazine, vol. 56, no. 7, pp. 21-27, 2018.

[86] Y. Li, Z. Zhang, W. Chen, Z. Feng, and M. F. Iskander, "A dualpolarization slot antenna using a compact CPW feeding structure," IEEE Antennas and Wireless Propagation Letters, vol. 9, pp. 191-194, 2010.

[87] J. L. Vazquez-Roy, V. Krozer, and J. Dall, "Wideband dual-polarization microstrip patch antenna array for airborne ice sounder," IEEE Antennas and Propagation Magazine, vol. 54, no. 4, pp. 98-107, 2012.

[88] F. Bongard, M. Gimersky, S. Doherty, X. Aubry, and M. Krummen, "3D-printed Ka-band waveguide array antenna for mobile SATCOM applications," in Antennas and Propagation (EUCAP), 2017 11th European Conference on. IEEE, 2017, pp. 579-583.

[89] S.-G. Zhou, G.-L. Huang, T.-H. Chio, J.-J. Yang, and G. Wei, "Design of a wideband dual-polarization full-corporate waveguide feed antenna 
array," IEEE Transactions on Antennas and Propagation, vol. 63, no. 11, pp. 4775-4782, 2015.

[90] X.-L. Lu, H. Zhang, S.-M. Gu, H. Liu, X.-C. Wang, and W.-Z. Lu, "A Dual-Polarized Cross-Slot Antenna Array on a Parallel-Plate Waveguide With Compact Structure and High Efficiency," IEEE Antennas and Wireless Propagation Letters, vol. 17, no. 1, pp. 8-11, 2018.

[91] S.-G. Zhou, G.-L. Huang, and T.-H. Chio, "A Lightweight, Wideband, Dual-Circular-Polarized Waveguide Cavity Array Designed With Direct Metal Laser Sintering Considerations," IEEE Transactions on Antennas and Propagation, vol. 66, no. 2, pp. 675-682, 2018.

[92] D. Kim, M. Zhang, J. Hirokawa, and M. Ando, "Design and fabrication of a dual-polarization waveguide slot array antenna with high isolation and high antenna efficiency for the $60 \mathrm{GHz}$ band," IEEE Transactions on Antennas and Propagation, vol. 62, no. 6, pp. 3019-3027, 2014.

[93] K. Tekkouk, J. Hirokawa, R. Sauleau, M. Ettorre, M. Sano, and M. Ando, "Dual-layer ridged waveguide slot array fed by a Butler matrix with sidelobe control in the 60-GHz band," IEEE Trans. Antennas Propag., vol. 63 , no. 9, pp. 3857-3867, 2015.

[94] K. Tekkouk, M. Ettorre, E. Gandini, and R. Sauleau, "Multibeam pillbox antenna with low sidelobe level and high-beam crossover in SIW technology using the split aperture decoupling method," IEEE Transactions on Antennas and Propagation, vol. 63, no. 11, pp. 5209-5215, 2015.

[95] K. Tekkouk, M. Ettorre, L. Le Coq, and R. Sauleau, "Multibeam SIW slotted waveguide antenna system fed by a compact dual-layer Rotman lens," IEEE Transactions on Antennas and Propagation, vol. 64, no. 2, pp. 504-514, 2016.

[96] S. A. Matos, E. B. Lima, J. S. Silva, J. R. Costa, C. A. Fernandes, N. J. Fonseca, and J. R. Mosig, "High gain dual-band beam-steering transmit array for Satcom terminals at Ka-band," IEEE Transactions on Antennas and Propagation, vol. 65, no. 7, pp. 3528-3539, 2017.

[97] E. B. Lima, S. A. Matos, J. R. Costa, C. A. Fernandes, and N. J. Fonseca, "Circular polarization wide-angle beam steering at Ka-band by in-plane translation of a plate lens antenna," IEEE Transactions on Antennas and Propagation, vol. 63, no. 12, pp. 5443-5455, 2015.

[98] J. Wan, S. Lu, X. Wang, and Y. Ai, "A steerable spot beam reflector antenna for geostationary satellites," IEEE Antennas and Wireless Propagation Letters, vol. 15, pp. 89-92, 2016. 
[99] E. Gandini, M. Ettorre, M. Casaletti, K. Tekkouk, L. Le Coq, and R. Sauleau, "SIW slotted waveguide array with pillbox transition for mechanical beam scanning," IEEE Antennas and Wireless Propagation Letters, vol. 11, pp. 1572-1575, 2012.

[100] M. Ettorre, F. F. Manzillo, M. Casaletti, R. Sauleau, L. Le Coq, and N. Capet, "Continuous transverse stub array for Ka-band applications," IEEE Transactions on Antennas and Propagation, vol. 63, no. 11, pp. 4792-4800, 2015.

[101] X. Yang, S. Xu, F. Yang, M. Li, Y. Hou, S. Jiang, and L. Liu, "A broadband high-efficiency reconfigurable reflectarray antenna using mechanically rotational elements," IEEE Transactions on Antennas and Propagation, vol. 65, no. 8, pp. 3959-3966, 2017.

[102] K. Tekkouk, J. Hirokawa, R. Sauleau, and M. Ando, "Wideband and Large Coverage Continuous Beam Steering Antenna in the $60-\mathrm{GHz}$ Band," IEEE Transactions on Antennas and Propagation, vol. 65, no. 9, pp. 4418-4426, 2017.

[103] M. Tripodi, F. DiMarca, T. Cadili, C. Mollura, F. DiMaggio, and M. Russo, "Ka band active phased array antenna system for satellite communication on the move terminal," in Satellite Telecommunications (ESTEL), 2012 IEEE First AESS European Conference on. IEEE, 2012, pp. $1-4$.

[104] A. Cherrette, R. D. Bruno, S. Brisbin, and G. Lok, "Hybrid electronic/mechanical scanning array antenna," Nov. 14 2017, uS Patent $9,819,082$.

[105] Y.-B. Jung, S.-Y. Eom, S.-I. Jeon, A. Shishlov, and C.-J. Kim, "Novel hybrid antenna design having a shaped reflector for mobile satellite communication applications," in Antennas and Propagation Society International Symposium (APSURSI), 2010 IEEE. IEEE, 2010, pp. 1-4.

[106] Y.-B. Jung, A. V. Shishlov, and S.-O. Park, "Cassegrain antenna with hybrid beam steering scheme for mobile satellite communications," IEEE Transactions on Antennas and Propagation, vol. 57, no. 5, pp. 1367-1372, 2009.

[107] P. Mousavi, M. Fakharzadeh, and S. Safavi-Naeini, "Design and implementation of a low cost adaptive antenna system for mobile satellite communication," in Phased Array Systems and Technology (ARRAY), 2010 IEEE International Symposium on. IEEE, 2010, pp. 512-517. 


\section{REFERENCES}

[108] F. Tiezzi, S. Vaccaro, D. Llorens, C. Dominguez, and M. Fajardo, "Kuband hybrid phased array antennas for mobile satellite communication systems," in Antennas and Propagation (EuCAP), 2013 7th European Conference on. IEEE, 2013, pp. 1605-1608.

[109] M. Shelley, J. Vazquez, and D. Moore, "X-and Ka-band low profile antennas for aeronautical and land mobile SATCOM," in Antennas and Propagation (EuCAP), 2014 8th European Conference on. IEEE, 2014, pp. 2619-2622.

[110] T. Lambard, O. Lafond, M. Himdi, H. Jeuland, S. Bolioli, and L. Le Coq, "Ka-band phased array antenna for high-data-rate SATCOM," IEEE antennas and wireless propagation letters, vol. 11, pp. 256-259, 2012.

[111] H. Zhou and F. Aryanfar, "A planar Ka-band phased array with configurable polarizations," in Antennas and Propagation Society International Symposium (APSURSI), 2013 IEEE. IEEE, 2013, pp. 1638-1639.

[112] Y. Zhang, J. Bai, S. Shi, and D. W. Prather, "Ka-band phased patch array antenna integrated with a PET-controlled phase shifter," International Journal of RF and Microwave Computer-Aided Engineering, vol. 26, no. 3, pp. 199-208, 2016.

[113] B. Rohrdantz, K. Kuhlmann, A. Stark, A. Geise, and A. F. Jacob, "Digital beamforming antenna array with polarisation multiplexing for mobile high-speed satellite terminals at Ka-band," The Journal of Engineering, vol. 2016, no. 6, pp. 180-188, 2016.

[114] E. Abdo-Sánchez, D. Palacios-Campos, C. Frías-Heras, F. Y. Ng-Molina, T. M. Martín-Guerrero, and C. Camacho-Peñalosa, "Electronically steerable and fixed-beam frequency-tunable planar traveling-wave antenna," IEEE Transactions on Antennas and Propagation, vol. 64, no. 4, pp. 1298-1306, 2016.

[115] G. Perez-Palomino, M. Barba, J. A. Encinar, R. Cahill, R. Dickie, P. Baine, and M. Bain, "Design and demonstration of an electronically scanned reflectarray antenna at $100 \mathrm{GHz}$ using multiresonant cells based on liquid crystals," IEEE Transactions on Antennas and Propagation, vol. 63 , no. 8, pp. 3722-3727, 2015.

[116] P.-S. Kildal, "Artificially soft and hard surfaces in electromagnetics," IEEE Transactions on Antennas and Propagation, vol. 38, no. 10, pp. $1537-1544,1990$.

[117] — - "Definition of artificially soft and hard surfaces for electromagnetic waves," Electronics Letters, vol. 24, no. 3, pp. 168-170, 1988. 
[118] D. Sievenpiper, L. Zhang, R. F. Broas, N. G. Alexopolous, and E. Yablonovitch, "High-impedance electromagnetic surfaces with a forbidden frequency band," IEEE Transactions on Microwave Theory and techniques, vol. 47, no. 11, pp. 2059-2074, 1999.

[119] P.-S. Kildal, A. A. Kishk, and A. Tengs, "Reduction of forward scattering from cylindrical objects using hard surfaces," IEEE Transactions on Antennas and Propagation, vol. 44, no. 11, pp. 1509-1520, 1996.

[120] D. Schurig, J. Mock, B. Justice, S. A. Cummer, J. B. Pendry, A. Starr, and D. Smith, "Metamaterial electromagnetic cloak at microwave frequencies," Science, vol. 314, no. 5801, pp. 977-980, 2006.

[121] P.-S. Kildal, A. Kishk, and Z. Sipus, "RF invisibility using metamaterials: Harry Potter's cloak or the emperor's new clothes?" in Antennas and Propagation Society International Symposium, 2007 IEEE. IEEE, 2007, pp. 2361-2364.

[122] P. P. de la Torre, J. Fernández, and M. Sierra-Castaner, "Parallel plate waveguide with AMC-PEC-AMC strips," in Antennas and Propagation, 2006. EuCAP 2006. First European Conference on. IEEE, 2006, pp. $1-5$.

[123] A. Valero-Nogueira and E. Alfonso, "How gap waveguides were conceived," in Antennas and Propagation (EUCAP), 2017 11th European Conference on. IEEE, 2017, pp. 242-246.

[124] A. Valero-Nogueira, E. Alfonso, J. Herranz, and M. Baquero, "Planar slot-array antenna fed by an oversized quasi-TEM waveguide," Microwave and Optical Technology Letters, vol. 49, no. 8, pp. 1875-1877, 2007.

[125] P.-S. Kildal and A. Kishk, "EM Modeling of surfaces with STOP or GO characteristics-artificial magnetic conductors and soft and hard surfaces," Applied Computational Electromagnetics Society Journal, vol. 18, no. 1, pp. 32-40, 2003.

[126] P.-S. Kildal, "Waveguides and transmission lines in gaps between parallel conducting surfaces," Aug. 12 2014, uS Patent 8,803,638.

[127] E. Rajo-Iglesias and P.-S. Kildal, "Numerical studies of bandwidth of parallel-plate cut-off realised by a bed of nails, corrugations and mushroom-type electromagnetic bandgap for use in gap waveguides," IET microwaves, antennas 83 propagation, vol. 5, no. 3, pp. 282-289, 2011.

[128] E. Alfonso Alós et al., "New quasi-TEM waveguides using artificial surfaces and their application to antennas and circuits," 2013. 


\section{REFERENCES}

[129] E. Alfonso, M. Baquero, P.-S. Kildal, A. Valero-Nogueira, E. RajoIglesias, and J. I. Herranz, "Design of microwave circuits in ridge-gap waveguide technology," in Microwave Symposium Digest (MTT), 2010 IEEE MTT-S International. IEEE, 2010, pp. 1544-1547.

[130] M. Bosiljevac, Z. Sipus, and P.-S. Kildal, “Construction of Green's functions of parallel plates with periodic texture with application to gap waveguides-a plane-wave spectral-domain approach," IET microwaves, antennas $\&$ propagation, vol. 4, no. 11, pp. 1799-1810, 2010.

[131] A. Polemi and S. Maci, "Closed form expressions for the modal dispersion equations and for the characteristic impedance of a metamaterial-based gap waveguide," IET microwaves, antennas 8 propagation, vol. 4, no. 8, pp. 1073-1080, 2010.

[132] E. Alfonso, P.-S. Kildal, A. Valero-Nogueira, and J. I. Herranz, "Numerical analysis of a metamaterial-based ridge gap waveguide with a bed of nails as parallel-plate mode killer," in Antennas and Propagation, 2009. EuCAP 2009. 3rd European Conference on. IEEE, 2009, pp. 23-27.

[133] E. Alfonso, P.-S. Kildal, A. Valero-Nogueira, and M. Baquero, "Study of the characteristic impedance of a ridge gap waveguide," in Antennas and Propagation Society International Symposium, 2009. APSURSI'09. IEEE. IEEE, 2009, pp. 1-4.

[134] E. Alfonso, M. Baquero, A. Valero-Nogueira, J. Herranz, and P.-S. Kildal, "Power divider in ridge gap waveguide technology," in Antennas and Propagation (EuCAP), 2010 Proceedings of the Fourth European Conference on. IEEE, 2010, pp. 1-4.

[135] E. Alfonso, A. U. Zaman, E. Pucci, and P.-S. Kildal, "Gap waveguide components for millimetre-wave systems: Couplers, filters, antennas, MMIC packaging," in Antennas and Propagation (ISAP), 2012 International Symposium on. IEEE, 2012, pp. 243-246.

[136] E. Pucci, "Gap Waveguide Technology for Millimeter Wave Applications and Integration with Antennas," Ph.D. dissertation, Chalmers University of Technology, 2013.

[137] P.-S. Kildal, "Three metamaterial-based gap waveguides between parallel metal plates for mm/submm waves," in Antennas and Propagation, 2009. EuCAP 2009. 3rd European Conference on. IEEE, 2009, pp. 28-32.

[138] M. G. Silveirinha, C. A. Fernandes, and J. R. Costa, "Electromagnetic characterization of textured surfaces formed by metallic pins," IEEE 
Transactions on Antennas and Propagation, vol. 56, no. 2, pp. 405-415, 2008.

[139] A. U. Zaman and P.-S. Kildal, "Gap Waveguides," in Handbook of Antenna Technologies. Springer, 2016, pp. 3273-3347.

[140] E. Pucci, A. U. Zaman, E. Rajo-Iglesias, and P.-S. Kildal, "New low loss inverted microstrip line using gap waveguide technology for slot antenna applications," in Antennas and Propagation (EUCAP), Proceedings of the 5th European Conference on. IEEE, 2011, pp. 979-982.

[141] E. Pucci, A. U. Zaman, E. Rajo-Iglesias, P.-S. Kildal, and A. Kishk, "Losses in ridge gap waveguide compared with rectangular waveguides and microstrip transmission lines," in Antennas and Propagation (EuCAP), 2010 Proceedings of the Fourth European Conference on. IEEE, 2010, pp. 1-4.

[142] E. Pucci, P.-S. Kildal, and E. Rajo-Iglesias, "Evaluation of losses in microstrip gap waveguide for slot antennas applications," in Antennas and Propagation Society International Symposium (APSURSI), 2012 IEEE. IEEE, 2012, pp. 1-2.

[143] D. Sánchez-Escuderos, M. Ferrando-Bataller, A. Berenguer, M. BaqueroEscudero, and A. Valero-Nogueira, "Dielectric bed of nails in gapwaveguide technology at millimeter-wave frequencies," IEEE Microwave and Wireless Components Letters, vol. 24, no. 8, pp. 515-517, 2014.

[144] E. Pucci, A. U. Zaman, E. Rajo-Iglesias, P.-S. Kildal, and A. Kishk, "Study of Q-factors of ridge and groove gap waveguide resonators," IET Microwaves, Antennas 83 Propagation, vol. 7, no. 11, pp. 900-908, 2013.

[145] B. Rosas, "The design and test of broadband launches up to $50 \mathrm{GHz}$ on thin and thick substrates," Southwest Microwave Inc., Tempe, AZ, 2011.

[146] D. C. Thompson, O. Tantot, H. Jallageas, G. E. Ponchak, M. M. Tentzeris, and J. Papapolymerou, "Characterization of liquid crystal polymer (LCP) material and transmission lines on LCP substrates from 30 to $110 \mathrm{GHz}$," IEEE Transactions on Microwave Theory and Techniques, vol. 52, no. 4, pp. 1343-1352, 2004.

[147] A. A. Brazalez, E. Rajo-Iglesias, J. L. Vazquez-Roy, A. Vosoogh, and P.S. Kildal, "Design and validation of microstrip gap waveguides and their transitions to rectangular waveguide, for millimeter-wave applications," IEEE Transactions on Microwave Theory and Techniques, vol. 63, no. 12, pp. 4035-4050, 2015. 
[148] A. U. Zaman, P.-S. Kildal, and A. A. Kishk, "Narrow-band microwave filter using high-Q groove gap waveguide resonators with manufacturing flexibility and no sidewalls," IEEE Transactions on Components, Packaging and Manufacturing Technology, vol. 2, no. 11, pp. 1882-1889, 2012.

[149] S. Birgermajer, N. Janković, V. Radonić, V. Crnojević-Bengin, and M. Bozzi, "Microstrip-Ridge Gap Waveguide Filter Based on Cavity Resonators With Mushroom Inclusions," IEEE Transactions on Microwave Theory and Techniques, vol. 66, no. 1, pp. 136-146, 2018.

[150] M. Rezaee, A. U. Zaman, and P.-S. Kildal, "A groove gap waveguide iris filter for V-band application," in Electrical Engineering (ICEE), 2015 23rd Iranian Conference on. IEEE, 2015, pp. 462-465.

[151] A. Vosoogh, A. A. Brazález, and P.-S. Kildal, "A V-band inverted microstrip gap waveguide end-coupled bandpass filter," IEEE Microwave and Wireless Components Letters, vol. 26, no. 4, pp. 261-263, 2016.

[152] B. Ahmadi and A. Banai, "Direct coupled resonator filters realized by gap waveguide technology," IEEE Transactions on Microwave Theory and Techniques, vol. 63, no. 10, pp. 3445-3452, 2015.

[153] B. Al-Juboori, Y. Huang, D. Klugmann, M. Hussein, and J. Zhou, "Millimeter wave cross-coupled bandpass filter based on groove gap waveguide technology," in Millimetre Waves and Terahertz Technologies (UCMMT), 2017 10th UK-Europe-China Workshop on. IEEE, 2017, pp. 1-4.

[154] A. Berenguer, M. Baquero-Escudero, D. Sanchez-Escuderos, B. Bernardo-Clemente, and V. E. Boria-Esbert, "Low insertion loss 61 $\mathrm{GHz}$ narrow-band filter implemented with groove gap waveguides," in Microwave Conference (EuMC), 2014 44th European. IEEE, 2014, pp. 191-194.

[155] A. Berenguer, D. Sánchez-Escuderos, B. Bernardo-Clemente, M. Baquero-Escudero, and V. Boria, "Groove gap waveguide as an alternative to rectangular waveguide for H-plane components," Electronics Letters, vol. 52, no. 11, pp. 939-941, 2016.

[156] A. Berenguer, M. Baquero-Escudero, D. Sanchez-Escuderos, V. E. Boria, and F. Vico, "A procedure to correct the response of manufactured Groove Gap Waveguide components," in Antennas and Propagation (EuCAP), 2016 10th European Conference on. IEEE, 2016, pp. 1-5.

[157] M. S. Sorkherizi, A. Khaleghi, and P.-S. Kildal, "Direct-coupled cavity filter in ridge gap waveguide," IEEE Transactions on Components, Packaging and Manufacturing Technology, vol. 4, no. 3, pp. 490-495, 2014. 
[158] A. Berenguer, M. Baquero-Escudero, D. Sanchez-Escuderos, and F. Vico, "Suspended-strip gap waveguide coupled-line properties for Ka-band component design," in Antennas and Propagation (EuCAP), 2015 9th European Conference on. IEEE, 2015, pp. 1-5.

[159] E. A. Alós, A. U. Zaman, and P.-S. Kildal, "Ka-band gap waveguide coupled-resonator filter for radio link diplexer application," IEEE Transactions on Components, Packaging and Manufacturing Technology, vol. 3, no. 5, pp. 870-879, 2013.

[160] M. Rezaee, A. U. Zaman, and P.-S. Kildal, "V-band groove gap waveguide diplexer," in Antennas and Propagation (EuCAP), 2015 9th European Conference on. IEEE, 2015, pp. 1-4.

[161] H. S. Farahani, R. Sadeghzadeh, N. Gharanfeli, and A. A. Kishk, "Novel design of microwave diplexers using gap waveguide technology," $\mathrm{Mi}$ crowave and Optical Technology Letters, vol. 59, no. 5, pp. 1133-1136, 2017.

[162] S. I. Shams and A. A. Kishk, "Design of 3-dB Hybrid Coupler Based on RGW Technology," IEEE Transactions on Microwave Theory and Techniques, vol. 65, no. 10, pp. 3849-3855, 2017.

[163] M. M. M. Ali, S. I. Shams, and A.-R. Sebak, "Printed Ridge Gap Waveguide 3-dB Coupler: Analysis and Design Procedure," IEEE Access, 2017.

[164] S. Birgermajer, N. Janković, V. Crnojević-Bengin, M. Bozzi, and V. Radonić, "Forward-wave $0 \mathrm{~dB}$ directional coupler based on microstripridge gap waveguide technology," in Advanced Technologies, Systems and Services in Telecommunications (TELSIKS), 2017 13th International Conference on. IEEE, 2017, pp. 154-157.

[165] Z. Talepour and A. Khaleghi, "A K-band planar slot array antenna on a single layer ridge gap waveguide," in Antennas and Propagation (EUCAP), 2017 11th European Conference on. IEEE, 2017, pp. 1685-1689.

[166] D. Zarifi, A. Farahbakhsh, and A. Zaman, "A Ridge Gap Waveguide fed apperture-coupled microstrip antenna array for $60 \mathrm{GHz}$ applications," in Antennas and Propagation (EUCAP), 2017 11th European Conference on. IEEE, 2017, pp. 953-955.

[167] A. Farahbakhsh, D. Zarifi, and A. U. Zaman, "A mmWave Wideband Slot Array Antenna Based on Ridge Gap Waveguide with 30\% Bandwidth," IEEE Transactions on Antennas and Propagation, 2017. 


\section{REFERENCES}

[168] W. E. Frazier, "Metal additive manufacturing: a review," Journal of Materials Engineering and Performance, vol. 23, no. 6, pp. 1917-1928, 2014.

[169] E. Rajo-Iglesias, A. U. Zaman, and P.-S. Kildal, "Parallel plate cavity mode suppression in microstrip circuit packages using a lid of nails," IEEE Microwave and Wireless Components Letters, vol. 20, no. 1, pp. 31-33, 2010.

[170] A. U. Zaman, J. Yang, and P.-S. Kildal, "Using lid of pins for packaging of microstrip board for descrambling the ports of eleven antenna for radio telescope applications," in Antennas and Propagation Society International Symposium (APSURSI), 2010 IEEE. IEEE, 2010, pp. 1-4.

[171] A. A. Brazalez, A. U. Zaman, and P.-S. Kildal, "Improved microstrip filters using PMC packaging by lid of nails," IEEE Transactions on Components, Packaging and Manufacturing Technology, vol. 2, no. 7, pp. 10751084, 2012.

[172] E. Rajo-Iglesias, P.-S. Kildal, A. U. Zaman, and A. Kishk, "Bed of springs for packaging of microstrip circuits in the microwave frequency range," IEEE Transactions on Components, Packaging and Manufacturing Technology, vol. 2, no. 10, pp. 1623-1628, 2012.

[173] E. Rajo-Iglesias, E. Pucci, A. A. Kishk, and P.-S. Kildal, "Suppression of parallel plate modes in low frequency microstrip circuit packages using lid of printed zigzag wires," IEEE Microwave and Wireless Components Letters, vol. 23, no. 7, pp. 359-361, 2013.

[174] J. I. H. Herruzo, A. Valero-Nogueira, S. M. Giner, and A. V. Jiménez, "Untilted Narrow-Wall Slots Excited by Parasitic Dipoles in Groove Gap Waveguide Technology," IEEE Transactions on Antennas and Propagation, vol. 63, no. 11, pp. 4759-4765, 2015.

[175] A. J. Sáez, A. Valero-Nogueira, J. I. Herranz, and B. Bernardo, "Singlelayer cavity-backed slot array fed by groove gap waveguide," IEEE Antennas and Wireless Propagation Letters, vol. 15, pp. 1402-1405, 2016.

[176] B. Cao, H. Wang, and Y. Huang, "W-Band High-Gain TE_\{220\}-Mode Slot Antenna Array With Gap Waveguide Feeding Network," IEEE Antennas and Wireless Propagation Letters, vol. 15, pp. 988-991, 2016.

[177] H. Attia, M. S. Sorkherizi, and A. A. Kishk, "60 GHz slot antenna array based on ridge gap waveguide technology enhanced with dielectric superstrate," in Antennas and Propagation (EuCAP), 2015 9th European Conference on. IEEE, 2015, pp. 1-4. 
[178] M. Al Sharkawy and A. A. Kishk, "Long slots array antenna based on ridge gap waveguide technology," IEEE Transactions on Antennas and Propagation, vol. 62, no. 10, pp. 5399-5403, 2014.

[179] P. Liu, A. U. Zaman, and P.-S. Kildal, "Design of a double layer cavity backed slot array antenna in gap waveguide technology," in Antennas and Propagation (ISAP), 2016 International Symposium on. IEEE, 2016, pp. 682-683.

[180] J. Liu, A. Vosoogh, A. U. Zaman, and P.-S. Kildal, "Design of a cavitybacked slot array unit cell on inverted microstrip gap waveguide," in $A n$ tennas and Propagation (ISAP), 2015 International Symposium. IEEE, 2015, pp. $1-4$.

[181] A. Farahbakhsh, D. Zarifi, and A. Zaman, "Ridge gap waveguide slot antenna array with $30 \%$ bandwidth for $60-\mathrm{GHz}$ applications," in Antennas and Propagation (EUCAP), 2017 11th European Conference on. IEEE, 2017, pp. 3050-3052.

[182] M. Ferrando-Rocher, A. Zaman, J. Yang, and A. Valero-Nogueira, "A dual-polarized slotted-waveguide antenna based on gap waveguide technology," in Antennas and Propagation (EUCAP), 2017 11th European Conference on. IEEE, 2017, pp. 3726-3727.

[183] N. Bayat-Makou and A. A. Kishk, "Millimeter-Wave Substrate Integrated Dual Level Gap Waveguide Horn Antenna," IEEE Transactions on Antennas and Propagation, vol. 65, no. 12, pp. 6847-6855, 2017.

[184] W. Huang, S. Chai, K. Xiao, and L. Ding, "An aperture coupled 5element microstrip patch array for $35 \mathrm{GHz}$ with ridge gap waveguide feeding," in Electromagnetics: Applications and Student Innovation Competition (iWEM), 2017 International Workshop on. IEEE, 2017, pp. 95-96.

[185] H. Wang, R. Gao, Y. Gu, J. Cao, and Z. Ye, "Low sidelobe leaky wave antenna based on gap waveguide technology," in Applied Computational Electromagnetics Society Symposium (ACES), 2017 International. IEEE, 2017, pp. 1-2.

[186] X. Dong, H. Wang, F. Xue, and Y. Liu, "Design and Measurement of a Novel Seamless Scanning Leaky Wave Antenna in Ridge Gap Waveguide Technology," Progress In Electromagnetics Research, vol. 58, pp. 147157, 2017.

[187] L. F. Carrera-Suárez, D. V. Navarro-Méndez, M. Baquero-Escudero, and A. Valero-Nogueira, "Rotman lens with Ridge-Gap Waveguides, implemented in LTCC technology, for $60 \mathrm{GHz}$ applications," in Antennas and 
Propagation (EuCAP), 2015 9th European Conference on. IEEE, 2015, pp. 1-5.

[188] H. Kirino and K. Ogawa, "A 76 GHz multi-layered phased array antenna using a non-metal contact metamaterial waveguide," IEEE Transactions on Antennas and Propagation, vol. 60, no. 2, pp. 840-853, 2012.

[189] S. M. Giner, A. Valero-Nogueira, J. I. H. Herruzo, and M. B. Escudero, "Excitation of untilted narrow-wall slot in groove gap waveguide by using a parasitic dipole," in Antennas and Propagation (EuCAP), 2013 7th European Conference on. IEEE, 2013, pp. 3082-3085.

[190] K. Ho and S. Newman, "State of the art electrical discharge machining (EDM)," International Journal of Machine Tools and Manufacture, vol. 43, no. 13, pp. 1287-1300, 2003.

[191] D. Zarifi, A. Farahbakhsh, and A. U. Zaman, "A Gap Waveguide-Fed Wideband Patch Antenna Array for 60-GHz Applications," IEEE Transactions on Antennas and Propagation, vol. 65, no. 9, pp. 4875-4879, 2017.

[192] O. Luukkonen, A. O. Karilainen, J. Vehmas, C. Simovski, and S. A. Tretyakov, "A high-impedance surface based antenna - Lose the antenna," in Antennas and Propagation (EuCAP), 2010 Proceedings of the Fourth European Conference on. IEEE, 2010, pp. 1-5.

[193] A. Tamayo-Domínguez, J.-M. Fernández-González, and M. SierraPérez, "Groove gap waveguide in metallized 3D-printed plastic and in mechanized aluminium in ka band," in Antennas and Propagation \& USNC/URSI National Radio Science Meeting, 2017 IEEE International Symposium on. IEEE, 2017, pp. 247-248.

[194] E. Pucci, E. Rajo-Iglesias, and P.-S. Kildal, "New microstrip gap waveguide on mushroom-type EBG for packaging of microwave components," IEEE Microwave and Wireless Components Letters, vol. 22, no. 3, p. 129, 2012.

[195] N. Kushwaha and R. Kumar, "Study of different shape electromagnetic band gap (EBG) structures for single and dual band applications," Journal of Microwaves, Optoelectronics and Electromagnetic Applications, vol. 13, no. 1, pp. 16-30, 2014.

[196] H. Kumar, M. Kumar, M. Kumar, A. Kumar, and R. Kanth, "Study on band gap behaviour of electromagnetic band-gap (EBG) structure with microstrip antenna," in Advanced Communication Technology (ICACT), 2012 14th International Conference on. IEEE, 2012, pp. 356-359. 
[197] C.-D. Wang and T.-L. Wu, "Model and mechanism of miniaturized and stopband-enhanced interleaved EBG structure for power/ground noise suppression," IEEE Transactions on Electromagnetic Compatibility, vol. 55, no. 1, pp. 159-167, 2013.

[198] E. Rajo-Iglesias, L. Inclan-Sanchez, J.-L. Vazquez-Roy, and E. GarciaMunoz, "Size reduction of mushroom-type EBG surfaces by using edgelocated vias," IEEE Microwave and Wireless Components Letters, vol. 17, no. 9, pp. 670-672, 2007.

[199] F. Yang and Y. Rahmat-Samii, Electromagnetic band gap structures in antenna engineering. Cambridge university press Cambridge, UK:, 2009.

[200] N. Jaglan, S. D. Gupta, S. Srivastava, and B. K. Kanaujia, "Satellite downlink communication band notched UWB antenna using uniplanar EBG structure," in Signal Processing and Communication (ICSC), 2016 International Conference on. IEEE, 2016, pp. 89-94.

[201] F.-R. Yang, K.-P. Ma, Y. Qian, and T. Itoh, "A uniplanar compact photonic-bandgap (UC-PBG) structure and its applications for microwave circuit," IEEE Transactions on microwave theory and techniques, vol. 47, no. 8, pp. 1509-1514, 1999.

[202] N. Jaglan and S. D. Gupta, "Design and development of band notched UWB circular monopole antenna with uniplanar star shaped EBG structures," International Journal of Microwave and Optical Technology (IJMOT), vol. 11, no. 2, pp. 86-91, 2016.

[203] Y. Toyota, A. E. Engin, T. H. Kim, and M. Swaminathan, "Stopband analysis using dispersion diagram for two-dimensional electromagnetic bandgap structures in printed circuit boards," IEEE microwave and wireless components letters, vol. 16, no. 12, pp. 645-647, 2006.

[204] M. Ebrahimpouri, O. Quevedo-Teruel, and E. Rajo-Iglesias, "Design guidelines for gap waveguide technology based on glide-symmetric holey structures," IEEE microwave and wireless components letters, vol. 27, no. 6 , pp. $542-544,2017$.

[205] M. Ebrahimpouri, E. Rajo-Iglesias, Z. Sipus, and O. Quevedo-Teruel, "Cost-Effective Gap Waveguide Technology Based on Glide-Symmetric Holey EBG Structures," IEEE Transactions on Microwave Theory and Techniques, 2017.

[206] O. Quevedo-Teruel, M. Ebrahimpouri, and M. N. M. Kehn, "Ultrawideband metasurface lenses based on off-shifted opposite layers," IEEE Antennas and Wireless Propagation Letters, vol. 15, pp. 484-487, 2016. 
[207] M. Ebrahimpouri, E. Rajo-Iglesias, and O. Quevedo-Teruel, "Wideband glide-symmetric holey structures for gap-waveguide technology," in $A n$ tennas and Propagation (EUCAP), 2017 11th European Conference on. IEEE, 2017, pp. 1658-1660.

[208] J. I. Herranz-Herruzo, A. Valero-Nogueira, M. Ferrando-Rocher, B. Bernardo, A. Vila, and R. Lenormand, "Low-Cost Ka-band Switchable RHCP/LHCP Antenna Array for Mobile SATCOM Terminal," IEEE Transactions on Antennas and Propagation, vol. 66, no. 5, pp. 26612666, 2018.

[209] E. Rajo-Iglesias and P.-S. Kildal, "Groove gap waveguide: A rectangular waveguide between contactless metal plates enabled by parallel-plate cutoff," in Antennas and Propagation (EuCAP), 2010 Proceedings of the Fourth European Conference on. IEEE, 2010, pp. 1-4.

[210] A. Valero-Nogueira, J. I. Herranz-Herruzo, M. Baquero, R. HernandezMurcia, and V. Rodrigo, "Practical derivation of slot equivalent admittance in periodic waveguides," IEEE Transactions on Antennas and Propagation, vol. 61, no. 4, pp. 2321-2324, 2013.

[211] M. Ferrando-Rocher, A. Valero-Nogueira, J. I. Herranz-Herruzo, A. Berenguer, and B. Bernardo-Clemente, "Groove Gap Waveguides: A contactless solution for multilayer slotted-waveguide array antenna assembly," in Antennas and Propagation (EuCAP), 2016 10th European Conference on. IEEE, 2016, pp. 1-4.

[212] A. Berenguer, V. Fusco, M. Ferrando-Rocher, and V. E. Boria, "A fast analysis method for the groove gap waveguide using transmission line theory," in Antennas and Propagation (EuCAP), 2016 10th European Conference on. IEEE, 2016, pp. 1-5.

[213] A. Farahbakhsh, D. Zarifi, A. Zaman, and P.-S. Kildal, "Corporate distribution networks for slot array antenna based on groove gap waveguide technology," in Antennas and Propagation (EuCAP), 2016 10th European Conference on. IEEE, 2016, pp. 1-3.

[214] J. I. Herranz-Herruzo, A. Valero-Nogueira, M. Ferrando-Rocher, B. Bernardo, A. Vila, and R. Lenormand, "Low-cost Ka-band Switchable RHCP/LHCP Antenna Array for Mobile SATCOM Terminal," IEEE Transactions on Antennas and Propagation, vol. PP, no. 99, pp. 1-1, 2018.

[215] S. A. Razavi, P.-S. Kildal, L. Xiang, H. Chen, and E. Alfonso, "Design of $60 \mathrm{GHz}$ planar array antennas using PCB-based microstrip-ridge gap 
waveguide and SIW," in Antennas and Propagation (EuCAP), 2014 8th European Conference on. IEEE, 2014, pp. 1825-1828.

[216] A. U. Zaman and P.-S. Kildal, "Ku band linear slot-array in ridge gapwaveguide technology," in Antennas and Propagation (EuCAP), 2013 7th European Conference on. IEEE, 2013, pp. 3078-3081.

[217] — - "A new $2 \times 2$ microstrip patch sub-array for $60 \mathrm{GHz}$ wideband planar antenna with ridge gap waveguide distribution layer," in Antennas and Propagation (EuCAP), 2015 9th European Conference on. IEEE, 2015, pp. 1-4.

[218] J. Liu, A. Vosoogh, A. U. Zaman, and J. Yang, "A high-gain highefficiency corporate-fed slot array antenna directly fed by ridge gap waveguide at $60-\mathrm{GHz}$," in 2017 International Symposium on Antennas and Propagation (ISAP), Oct 2017, pp. 1-2.

[219] M. Ferrando-Rocher, A. Valero-Nogueira, J. I. Herranz-Herruzo, and A. Berenguer, "V-band single-layer slot array fed by ridge gap waveguide," in Antennas and Propagation (APSURSI), 2016 IEEE International Symposium on. IEEE, 2016, pp. 389-390.

[220] M. Ferrando-Rocher, A. Valero-Nogueira, and J. I. Herranz-Herruzo, "New feeding network topologies for high-gain single-layer slot array antennas using gap waveguide concept," in Antennas and Propagation (EUCAP), 2017 11th European Conference on. IEEE, 2017, pp. 1654-1657.

[221] W. L. Stutzman and G. A. Thiele, Antenna theory and design. John Wiley \& Sons, 2012.

[222] W. H. Watson, The physical principles of wave guide transmission and antenna systems. The Clarendon press, 1947.

[223] A. Stevenson, "Theory of Slots in Rectangular Wave-Guides," Journal of Applied Physics, vol. 19, no. 1, pp. 24-38, 1948.

[224] M. Orefice and R. Elliott, "Design of waveguide-fed series slot arrays," in IEE Proceedings H (Microwaves, Optics and Antennas), vol. 129, no. 4. IET, 1982, pp. 165-169.

[225] F.-C. Chen, J.-M. Qiu, S.-W. Wong, and Q.-X. Chu, "Dual-band coaxial cavity bandpass filter with helical feeding structure and mixed coupling," IEEE Microwave and Wireless Components Letters, vol. 25, no. 1, pp. $31-33,2015$.

[226] H.-Y. Li, J.-X. Xu, X. Y. Zhang, and X.-L. Zhao, "Low-loss Narrowband Filtering Switch Based on Coaxial Resonators," IEEE Access, 2018. 
[227] Q.-Y. Lu, W. Qin, and J.-X. Chen, "A novel balanced bandpass filter based on twin-coaxial resonator," IEEE Microwave and Wireless Components Letters, vol. 27, no. 2, pp. 114-116, 2017.

[228] A. M. Sabaawi and C. C. Tsimenidis, "Circular truncated patch antennas for energy harvesting applications," in Antennas and Propagation Conference (LAPC), 2013 Loughborough. IEEE, 2013, pp. 369-373.

[229] S. K. Yong and C.-C. Chong, "An overview of multigigabit wireless through millimeter wave technology: Potentials and technical challenges," EURASIP journal on wireless communications and networking, vol. 2007, no. 1, pp. 50-50, 2007.

[230] D. Dogan and C. B. Top, "Circularly polarized Ka-band waveguide slot array with low sidelobes," in Antennas and Propagation (EUCAP), 2012 6th European Conference on. IEEE, 2012, pp. 1105-1109.

[231] M. Ferrando-Rocher, J. I. Herranz, A. Valero-Nogueira, and B. Bernardo, "Performance Assessment of Gap Waveguide Array Antennas: CNC Milling vs. 3D Printing," IEEE Antennas and Wireless Propagation Letters, 2018.

[232] M. Ferrando-Rocher, J. I. Herranz-Herruzo, A. Valero-Nogueira, and M. Baquero-Escudero, "Flatness enhancement of Gap Waveguide Slot Arrays using a Ribbed-Grid Plate," in Antennas and Propagation (APSURSI), 2018 IEEE International Symposium on. IEEE, 2018.

[233] C. Guo, X. Shang, M. J. Lancaster, and J. Xu, "A 3-D printed lightweight $\mathrm{X}$-band waveguide filter based on spherical resonators," IEEE Microwave and Wireless Components Letters, vol. 25, no. 7, pp. 442-444, 2015.

[234] G. P. Le Sage, "3D printed waveguide slot array antennas," IEEE Access, vol. 4, pp. 1258-1265, 2016.

[235] M. Ferrando-Rocher, J. I. Herranz-Herruzo, A. Valero-Nogueira, and A. Vila-Jiménez, "Single-Layer Circularly-Polarized Ka-Band Antenna using Gap Waveguide technology," IEEE Transactions on Antennas and Propagation, 2018. 